\title{
CanadianJournal of HealthTechnologies
}

March 2022 Volume 2 Issue 03

\section{CADTH Reimbursement Review}

\section{Mecasermin (Increlex)}

Sponsor: Ipsen Biopharmaceuticals Canada, Inc.

Therapeutic area: Severe primary insulin-like growth factor 1 deficiency 


\section{ISSN: $2563-6596$}

Disclaimer: The information in this document is intended to help Canadian health care decision-makers, health care professionals, health systems leaders, and policy-makers make well-informed decisions and thereby improve the quality of health care services. While patients and others may access this document, the document is made available for informational purposes only and no representations or warranties are made with respect to its fitness for any particular purpose. The information in this document should not be used as a substitute for professional medical advice or as a substitute for the application of clinical judgment in respect of the care of a particular patient or other professional judgment in any decision-making process. The Canadian Agency for Drugs and Technologies in Health (CADTH) does not endorse any information, drugs, therapies, treatments, products, processes, or services.

While care has been taken to ensure that the information prepared by CADTH in this document is accurate, complete, and up-to-date as at the applicable date the material was first published by CADTH, CADTH does not make any guarantees to that effect. CADTH does not guarantee and is not responsible for the quality, currency, propriety, accuracy, or reasonableness of any statements, information, or conclusions contained in any third-party materials used in preparing this document. The views and opinions of third parties published in this document do not necessarily state or reflect those of CADTH.

CADTH is not responsible for any errors, omissions, injury, loss, or damage arising from or relating to the use (or misuse) of any information, statements, or conclusions contained in or implied by the contents of this document or any of the source materials.

This document may contain links to third-party websites. CADTH does not have control over the content of such sites. Use of third-party sites is governed by the third-party website owners' own terms and conditions set out for such sites. CADTH does not make any guarantee with respect to any information contained on such third-party sites and CADTH is not responsible for any injury, loss, or damage suffered as a result of using such third-party sites. CADTH has no responsibility for the collection, use, and disclosure of personal information by third-party sites.

Subject to the aforementioned limitations, the views expressed herein are those of CADTH and do not necessarily represent the views of Canada's federal, provincial, or territorial governments or any third party supplier of information.

This document is prepared and intended for use in the context of the Canadian health care system. The use of this document outside of Canada is done so at the user's own risk.

This disclaimer and any questions or matters of any nature arising from or relating to the content or use (or misuse) of this document will be governed by and interpreted in accordance with the laws of the Province of Ontario and the laws of Canada applicable therein, and all proceedings shall be subject to the exclusive jurisdiction of the courts of the Province of Ontario, Canada.

The copyright and other intellectual property rights in this document are owned by CADTH and its licensors. These rights are protected by the Canadian Copyright Act and other national and international laws and agreements. Users are permitted to make copies of this document for non-commercial purposes only, provided it is not modified when reproduced and appropriate credit is given to CADTH and its licensors.

About CADTH: CADTH is an independent, not-for-profit organization responsible for providing Canada's health care decision-makers with objective evidence to help make informed decisions about the optimal use of drugs, medical devices, diagnostics, and procedures in our health care system.

Funding: CADTH receives funding from Canada's federal, provincial, and territorial governments, with the exception of Quebec. 


\section{Table of Contents}

Clinical Review

List of Tables 6

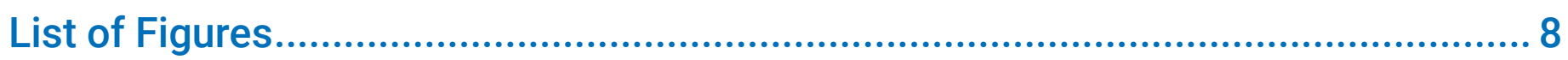

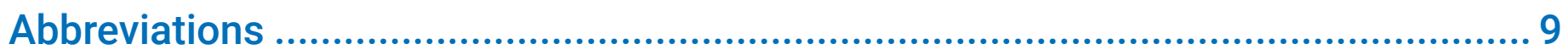

Executive Summary ............................................................................................ 10

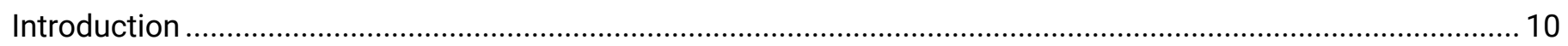

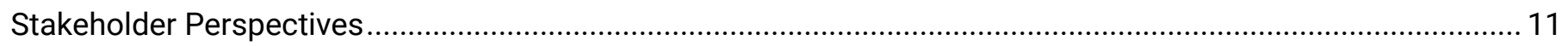

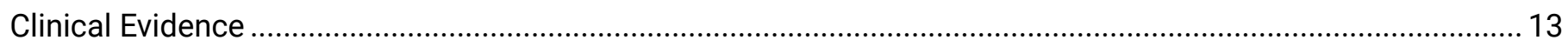

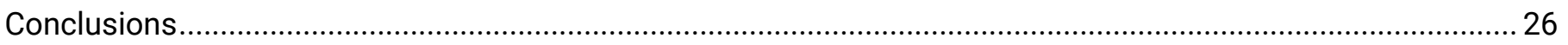

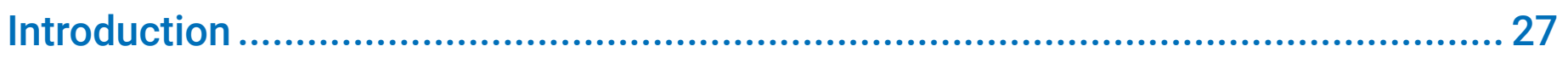

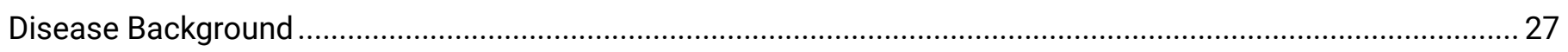

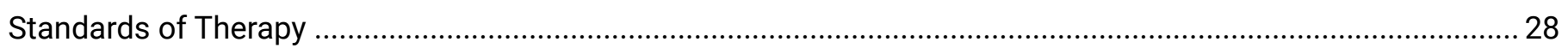

Drug

Stakeholder Perspectives......................................................................... 29

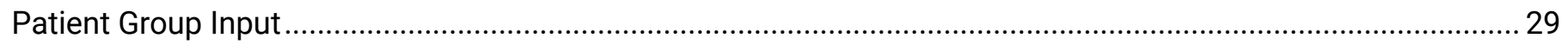

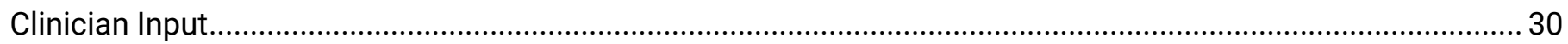

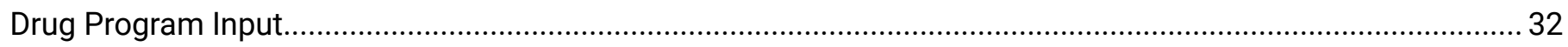

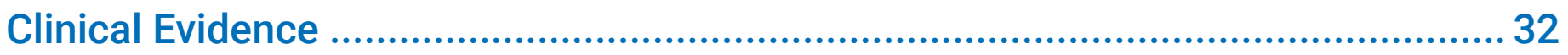

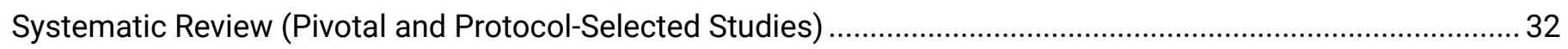

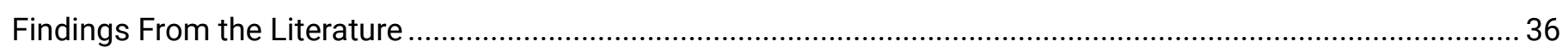

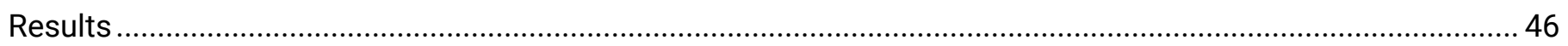

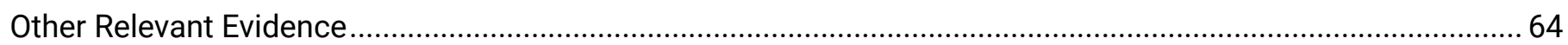

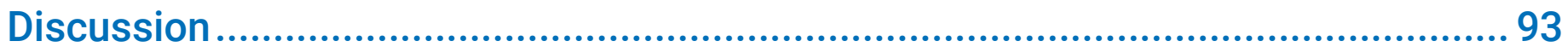

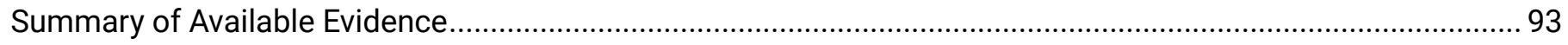

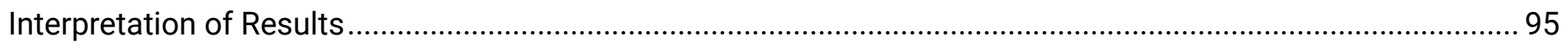

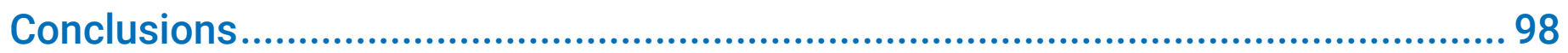

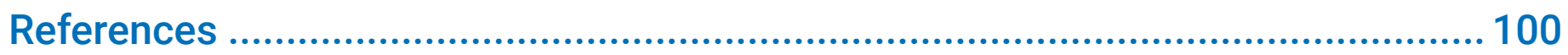


Appendix 1: Literature Search Strategy ............................................................. 102

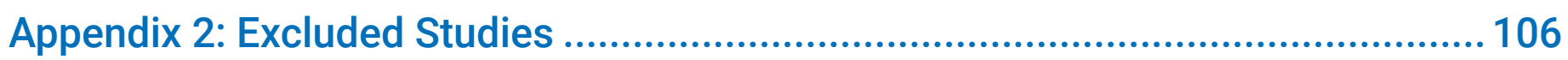

Pharmacoeconomic Review ............................................................ 109

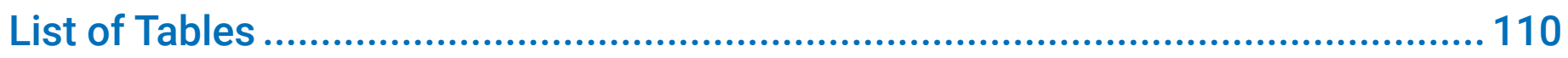

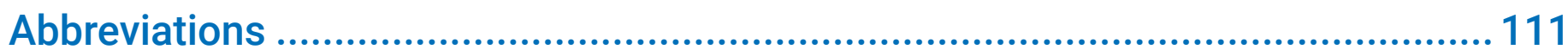

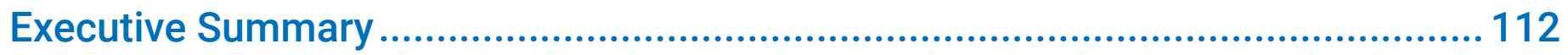

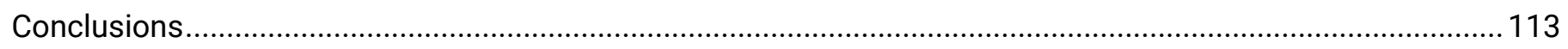

Stakeholder Input Relevant to the Economic Review ............................................ 114

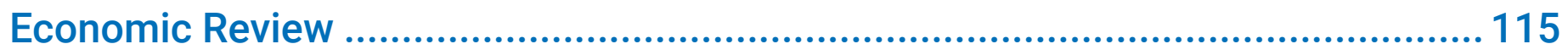

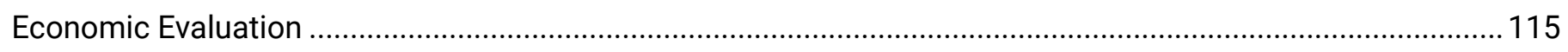

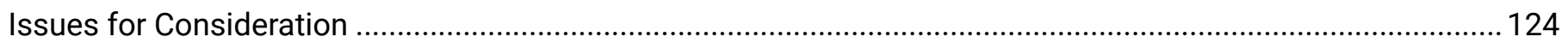

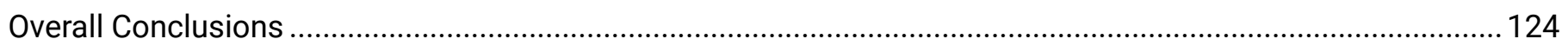

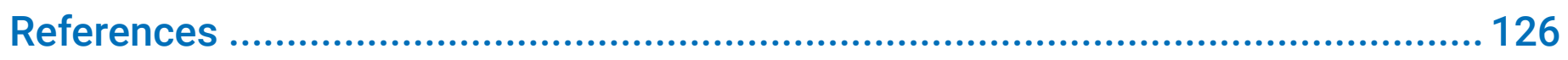

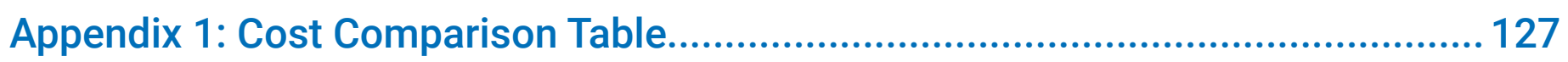

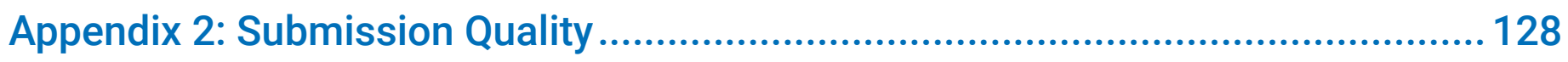

Appendix 3: Additional Information on the Submitted Economic Evaluation ..........129

Appendix 4: Additional Details on the CADTH Reanalyses and Sensitivity Analyses of the Economic Evaluation ............................................................................... 130

Appendix 5: Submitted Budget Impact Analysis and CADTH Appraisal ................. 132 


\section{List of Tables}

Table 1: Submitted for Review 10

Table 2: Summary of Key Results After 1 Year of Treatment in Study 1419 and Reports of Harms During Treatment

Table 3: Summary of Key Results After 1 Year of Treatment in the EU-IGFD Registry and Reports of Harms During Treatmenta 22

Table 4: Summary of Drug Plan Input and Clinical Expert Response...... 33

Table 5: Inclusion Criteria for the Systematic Review . 35

Table 6: Details of Study 1419 38

Table 7: Summary of Baseline Characteristics for Study 1419 .41

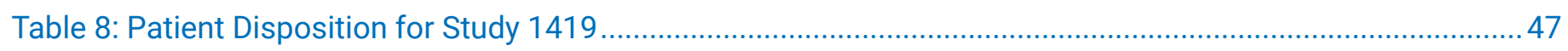

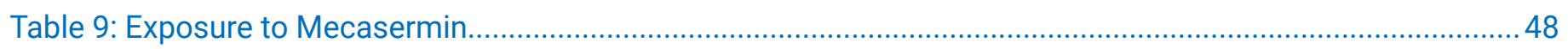

Table 10: Height Velocity Among Patients Naive to Mecasermin in Study 1419 ................................................51

Table 11: Height Velocity SDS Among Patients Naive to Mecasermin in Study 1419 .......................................5 54

Table 12: Height SDS Among Patients Naive to Mecasermin Treatment in Study 1419 .......................................5

Table 13: Change in Bone Age Relative to Chronological Age for Patients Naive to Mecasermin Treatment Who

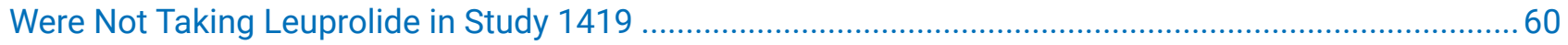

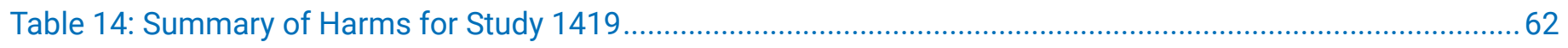

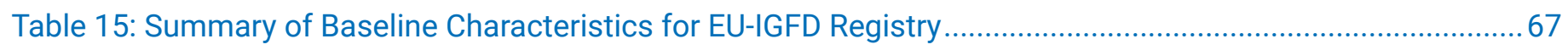

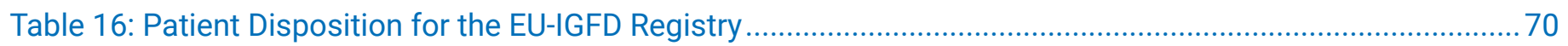

Table 17: Height During the First 5 Years of Treatment in the EU-IGFD Registry...............................................72

Table 18: Height During the First 5 Years of Treatment in the EU-IGFD Registry Among Patients With

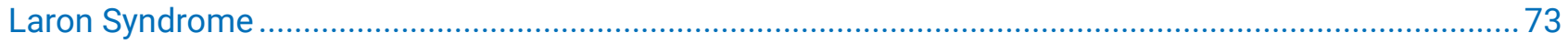

Table 19: Height SDS During the First 5 Years of Treatment in the EU-IGFD Registry........................................... 75

Table 20: Height SDS During the First 5 Years of Treatment in the EU-IGFD Registry Among Patients With and

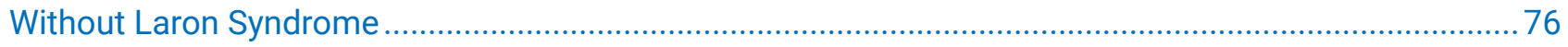

Table 21: Height Velocity During the First 5 Years of Treatment in the EU-IGFD Registry ....................................... 77

Table 22: Height Velocity During the First 5 Years of Treatment in the EU-IGFD Registry Among Patients With and Without Laron Syndrome...

Table 23: Predicted Adult Height and Final Adult Height in the EU-IGFD Registry Among Patients Who Achieved Adult Height.

Table 24: Bone Age During the First 5 Years of Treatment in the EU-IGFD Registry

Table 25: Difference Between Bone Age and Chronological Age During the First 3 Years of Treatment in the EU-IGFD Registry

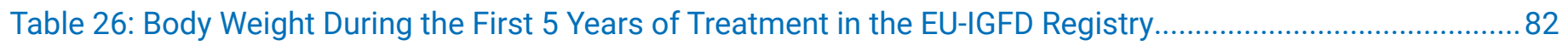

Table 27: Body Weight SDS During the First 5 Years of Treatment in the EU-IGFD Registry. .83 
Table 28: BMI SDS During the First 5 Years of Treatment in the EU-IGFD Registry ...........................................8 84

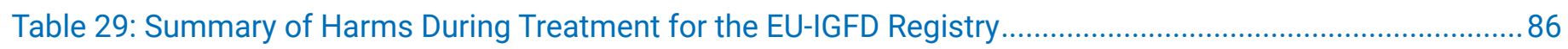

Table 30: Summary of Baseline Characteristics for the Polish Study on Increlex................................................89

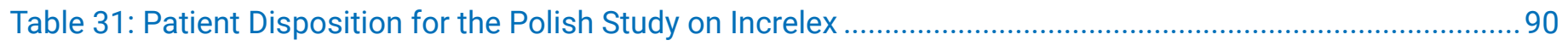

Table 32: Height SDS and Height Velocity After Three Years of Treatment in the Polish Study on Increlex ...........91

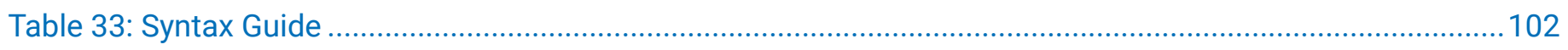

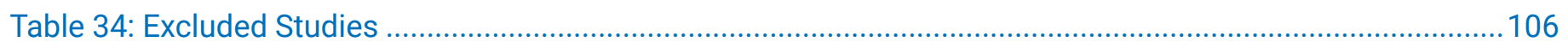




\section{List of Figures}

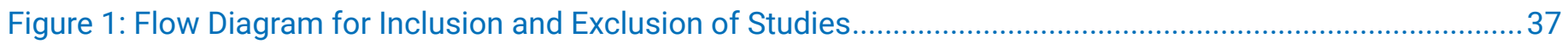

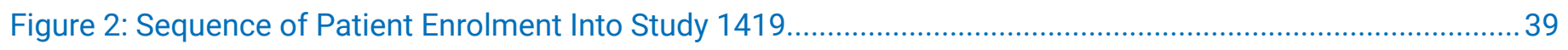

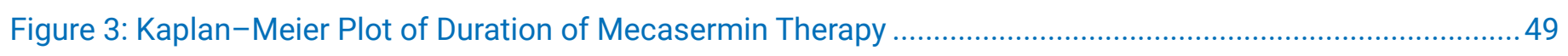

Figure 4: Year 1 Height Velocity Versus Dose of Mecasermin Per Injection in Study 1419..................................53

Figure 5: Cumulative Change in Bone Age Versus Cumulative Change in Chronological Age for Patients Naive to

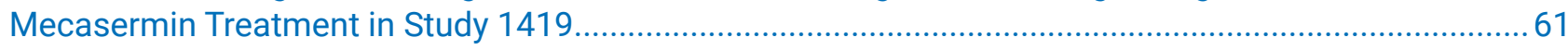




\section{Abbreviations}

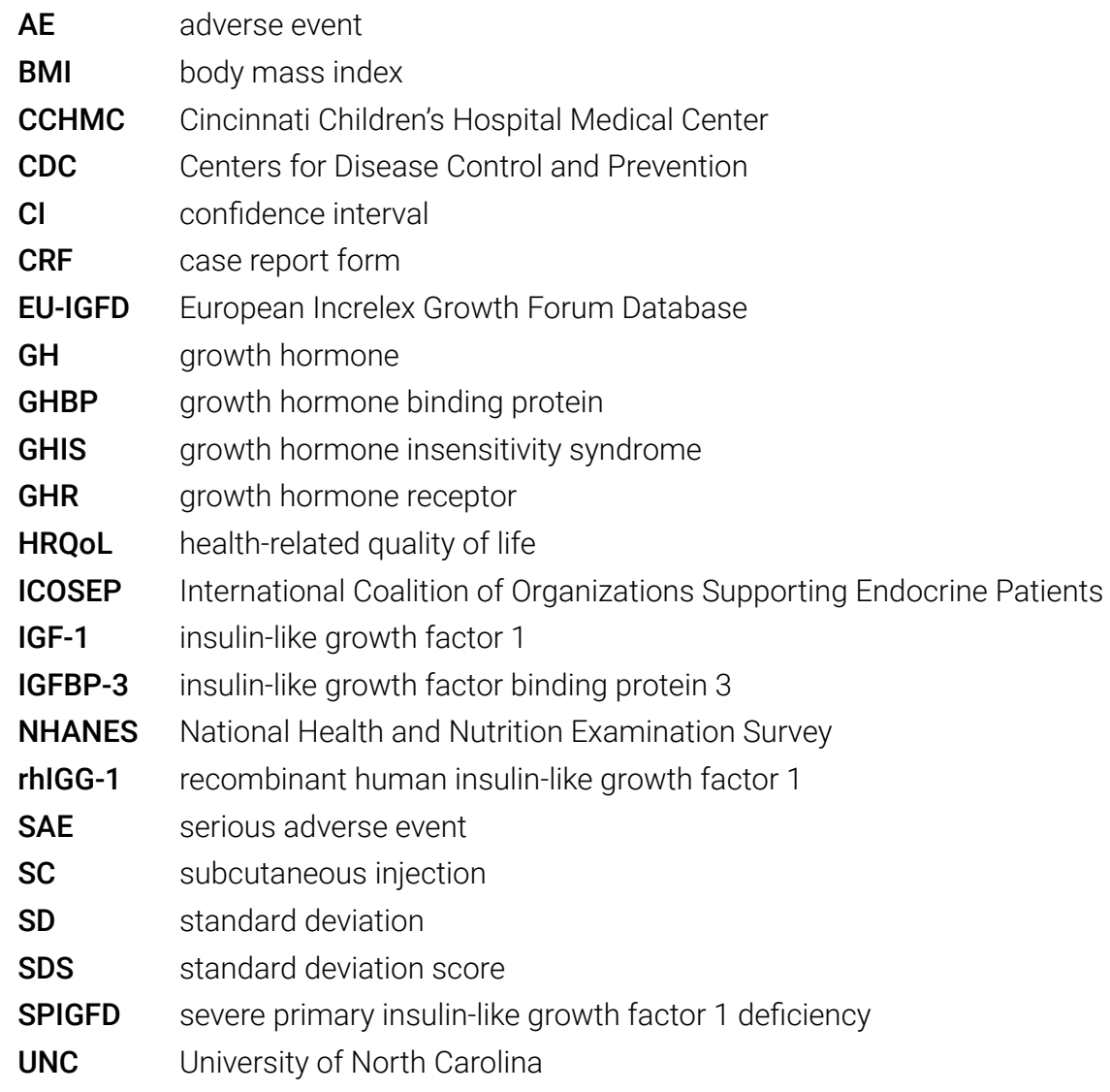




\section{Executive Summary}

An overview of the submission details for the drug under review is provided in Table 1.

\section{Introduction}

Severe primary insulin-like growth factor 1 deficiency (SPIGFD) is defined by a height standard deviation score (SDS) less than or equal to -3.0 , basal insulin-like growth factor 1 (IGF-1) levels below the 2.5th percentile for age and gender, growth hormone (GH) sufficiency, and the exclusion of secondary forms of IGF-1 deficiency. ${ }^{1}$ It includes patients with mutations in the growth hormone receptor (GHR), post-GHR signalling pathway, and IGF-1 gene defects. ${ }^{2,3}$ In Canada, it is estimated that approximately 4.88 cases of SPIGFD are diagnosed each year, or 1 case in every 77,000 births (for a prevalence of $0.0013 \%$ ). ${ }^{4-6}$

Laron syndrome is the most common known cause of SPIGFD. ${ }^{7}$ Clinical features of untreated Laron syndrome include severe post-natal growth failure (despite normal prenatal growth), short limb length (relative to trunk length), characteristic facial features (saddle nose and small forehead), delayed skeletal maturation and sexual development, small genitalia and testes, osteopenia, muscle weakness, obesity, and metabolic abnormalities (i.e., hyperlipidemia, hypoglycemia, and insulin resistance). ${ }^{8-11}$ Prior to the approval of mecasermin in Canada, there were no approved treatments for SPIGFD.

Table 1: Submitted for Review

\begin{tabular}{|c|c|}
\hline Item & Description \\
\hline Drug product & $\begin{array}{l}\text { Mecasermin (Increlex), } 10 \mathrm{mg} / \mathrm{mL} \text { ( } 40 \mathrm{mg} / 4 \mathrm{~mL} \text { vial), sterile solution for subcutaneous } \\
\text { injection }\end{array}$ \\
\hline Indication & $\begin{array}{l}\text { Treatment of growth failure in children and adolescents from } 2 \text { to } 18 \text { years with confirmed } \\
\text { SPIGFD } \\
\text { SPIGFD is defined by: } \\
\text { - height standard deviation score } \leq-3.0 \\
\text { - basal IGF-1 levels below the } 2.5 \text { th percentile for age and gender } \\
\text { - growth hormone sufficiency } \\
\text { - exclusion of secondary forms of IGF-1 deficiency, such as malnutrition, hypopituitarism, } \\
\text { hypothyroidism, or chronic treatment with pharmacologic doses of anti-inflammatory } \\
\text { steroids } \\
\text { SPIGFD includes patients with mutations in the GHR gene/Laron's syndrome, post-GHR } \\
\text { signalling pathway, and IGF-1 gene defects }\end{array}$ \\
\hline Reimbursement request & As per indication \\
\hline Health Canada approval status & NOC \\
\hline Health Canada review pathway & Standard \\
\hline NOC date & December 17, 2020 \\
\hline Sponsor & Ipsen Biopharmaceuticals Canada, Inc. \\
\hline
\end{tabular}

$\mathrm{GH}=$ growth hormone; GHR = growth hormone receptor; IGF-1 = insulin-like growth factor 1; NOC = Notice of Compliance; SPIGFD = severe primary insulin-like growth factor 1 deficiency. 
Mecasermin $10 \mathrm{mg} / \mathrm{mL}$ is indicated for children and adolescents from 2 to 18 years of age for the treatment of growth failure associated with SPIGFD. ${ }^{12}$ Mecasermin contains recombinant human insulin-like growth factor 1 (rhIGF-1), which is produced by recombinant DNA technology. The recommended starting dosage is $0.04 \mathrm{mg} / \mathrm{kg}$ to $0.08 \mathrm{mg} / \mathrm{kg}(40 \mathrm{mcg} / \mathrm{kg}$ to $80 \mathrm{mcg} / \mathrm{kg}$ ) by subcutaneous (SC) injection administered twice daily shortly before or after a meal or snack. The dose is tailored to each patient and adjusted based on tolerability and body weight. If well tolerated for at least 1 week, the dosage may be increased by $0.04 \mathrm{mg} / \mathrm{kg}$ SC twice daily to a maximum of $0.12 \mathrm{mg} / \mathrm{kg} \mathrm{SC}$ twice daily.

The objective was to perform a systematic review of the beneficial and harmful effects of mecasermin $10 \mathrm{mg} / \mathrm{mL} \mathrm{SC}$ injection for the treatment of growth failure in children and adolescents (aged 2 to 18 years) with confirmed SPIGFD.

\section{Stakeholder Perspectives}

The information in this section is a summary of input provided by the patient groups who responded to CADTH's call for patient input and from a clinical expert consulted by CADTH for the purpose of this review.

\section{Patient Input}

CADTH received 1 patient group submission from the International Coalition of Organizations Supporting Endocrine Patients (ICOSEP). The group emphasized the importance of diagnosing and treating children with SPIGFD early to reduce needless medical hardships over their lifetimes. The ICOSEP submission highlighted that, although short stature is the most visible symptom of SPIGFD, the consequences of the condition run deeper than just height and affect children's daily lives. For example, everyday activities such as getting out of bed, playing with others, and concentrating on tasks can take substantial effort. According to ICOSEP, the condition of children with SPIGFD who remain untreated will not improve, and patients may require a lifetime of specialized care if left untreated.

\section{Clinician Input \\ Input From the Clinical Expert Consulted by CADTH}

CADTH received input from a clinical specialist with expertise in the diagnosis and management of SPIGFD. The clinical expert indicated that, because there is no existing treatment for SPIGFD, mecasermin would be a first-line treatment for patients with either a clear diagnosis of SPIGFD or the presence of GH antibodies. The clinical expert noted that mecasermin is indicated when the clinical and biochemical criteria (a height SDS $\leq-3.0$; basal IGF-1 levels below the 2.5th percentile for age and sex; and GH sufficiency) are supported by a genetic diagnosis or the presence of $\mathrm{GH}$ antibodies (after ruling out other causes of short stature, such as nutritional causes and chronic diseases).

The clinical expert emphasized that a genetic diagnosis would be ideal before the use of mecasermin but acknowledged that this would cause a shift in the current treatment paradigm at the diagnostic level because access to the tests required for a definitive diagnosis of SPIGFD is limited in many Canadian jurisdictions. Further, not all genetic causes of SPIGFD are known, and currently less than half of the cases have an identifiable genetic variant. ${ }^{13}$ The clinical expert suggested that, if these clinical and biochemical criteria are not supported by a genetic diagnosis (mutations in the GHR gene [Laron syndrome], post-GHR signalling pathway dysfunction, and IGF-1 gene defects) or by the presence of GH antibodies, treatment decisions should be informed by a panel of clinical experts to avoid misdiagnosis 
and overtreatment. This may not be feasible as few Canadian clinicians have direct experience with the diagnosis and treatment of SPIGFD; however, physicians with expertise in managing pediatric endocrine growth disorders may also be qualified to contribute.

According to the clinical expert consulted by CADTH, patients likely to demonstrate a clinically meaningful response to mecasermin include those with SPIGFD due to a genetic defect of the GH-IGF-1 pathway, those who show biochemical evidence of inappropriate $\mathrm{GH}$ receptors, and those who have $\mathrm{GH}$ antibodies or $\mathrm{GH}$ resistance. The clinical expert stated that treatment should begin as early as possible to maximize gain in height. Treatment should be discontinued when the height velocity is less than $1 \mathrm{~cm}$ over 6 months or less than $2 \mathrm{~cm}$ over 1 year. Another indicator classically used for stopping treatment (in patients treated with $\mathrm{GH}$ for $\mathrm{GH}$ deficiency) is a bone age in the near-adult range (i.e., $>16$ years in males and $>14$ years in females). The clinical expert stated that the most important outcomes for assessing response to treatment are height velocity and final adult height.

\section{Drug Program Input}

Input was obtained from the drug programs that participate in the CADTH reimbursement review processes. The following were identified as key factors that could affect the implementation of a CADTH recommendation for mecasermin.

- There is a group of GH insensitivity syndromes that could respond to mecasermin, but also to relatively high doses of $\mathrm{GH}$. Is molecular testing for $\mathrm{GH}$ receptor gene mutations to definitively diagnose SPIGFD available across the country? Should eligibility criteria for mecasermin include a trial of 3 to 6 months of GH, unless SPIGFD is definitively diagnosed?

The clinical expert consulted by CADTH noted that patients with GH deficiency will likely respond to mecasermin. Conversely, patients with mild primary insulin-like growth factor deficiency may also respond to high doses of GH. Pediatric Endocrine Society guidelines recommend that patients with hormone-signalling defects known to be unresponsive to $\mathrm{GH}$ treatment start mecasermin directly; this includes patients with very low or undetectable levels of growth hormone binding protein (GHBP) and/or proven GHR mutations, $\mathrm{GH}$ neutralizing antibodies, and other known gene mutations associated with SPIGFD (e.g., STAT5 $b$ gene mutations and IGF-1 gene deletion or mutation). ${ }^{14}$ The clinical expert consulted by CADTH stated that molecular testing for GHR gene mutations (or other known mutations associated with SPIGFD) would be ideal; however, while molecular testing is available, it can be difficult to access in some Canadian jurisdictions and the cost is high. Moreover, molecular testing will always be limited to known genetic causes of SPIGFD. ${ }^{14}$ Pediatric Endocrine Society guidelines note that, to better inform a treatment plan, genetic testing is desirable for patients for whom diagnostic uncertainty is problematic. ${ }^{14}$ For patients with unexplained IGF-1 deficiency, the guidelines state that a trial of GH is reasonable; however, the guidelines do not provide recommendations for the length of the trial.

- Will leuprolide be used in clinical practice to delay puberty and prolong the growth period in an attempt to achieve a greater adult height?

The clinical expert consulted by CADTH noted that, although leuprolide is not indicated for this use in Canada and there is little scientific evidence that the addition of leuprolide to mecasermin would lead to a greater final height, some clinicians may choose to prescribe it for patients with SPIGFD to prolong the growth period. The decision to prescribe leuprolide 
would be made on a case-by-case basis. Usually, this would be reserved for patients who are close to their final adult height or for whom bone age is rapidly advancing.

- What height velocity is required to continue mecasermin treatment? At what point should mecasermin treatment be discontinued?

The clinical expert consulted by CADTH stated that treatment should be continued so long as there is an increase in height of at least $1 \mathrm{~cm}$ per 6 months or $2 \mathrm{~cm}$ per year. The decision to stop treatment should be based on bone age (i.e., $>16$ years for males and $>14$ years for females) rather than chronological age. At a bone age of $>16$ years for males and $>14$ years for females, patients are thought to have achieved at least $98 \%$ of their final adult height.

- Is there evidence that mecasermin provides a benefit for other clinical manifestations of Laron syndrome (aside from short stature)?

The clinical expert consulted by CADTH noted that there is currently no evidence from trials to suggest that mecasermin has any effect on other clinical manifestations of Laron syndrome. An observational study of 5 patients with Laron syndrome found that 3 to 10 months of treatment with rhIGF-1 resulted in a marked increase in head circumference, increased body weight, and a reduction in subcutaneous body fat. ${ }^{11}$

- Given warnings of benign and malignant neoplasms in patients treated with mecasermin, do the benefits of treatment outweigh the potential harms?

There are inadequate data to draw strong conclusions about the ratio of benefits to harms for patients treated with mecasermin for SPIGFD. In clinical practice, the ratio of benefits to harms needs to be discussed individually with each patient and their parent or caregiver. To make an informed decision, patients and their parents or caregivers should be told that the risk of benign and malignant tumours in children with SPIGFD is lower than it is for healthy children without SPIGFD (because IGF-1 plays a role in the initiation and progression of benign and malignant tumours). ${ }^{12}$ Treatment with mecasermin may increase the risk of benign and malignant tumours, although the relationship between mecasermin and the risk of benign and malignant tumours is uncertain.

A Canadian registry to monitor benefits and long-term harms in patients treated with mecasermin may be useful. The sponsor reported that, as of March 10, 2019, a total of 36 cases of benign or malignant neoplasms had been identified in its global safety database. In $67 \%$ of cases $(n=24$ of 36$)$, mecasermin was administered in an off-label indication. In $25 \%$ of cases ( $n=9$ of 36 ), mecasermin was used at a higher-than-recommended dose. For the 19 malignant neoplasia cases, $16(84 \%)$ reported an off-label use and $6(32 \%)$ reported an off-label dose. Because Health Canada requires mecasermin to be distributed through the sponsor's patient support program, the risk of off-label use and/or dosing higher than recommended is fully mitigated.

\section{Clinical Evidence}

Pivotal Study

Description of Study

One pivotal trial (Study 1419) was included. ${ }^{15-17}$ Study 1419 was a phase III open-label, multicentre, single-arm, investigator-sponsored trial with linked data from 4 predecessor studies (F0206S, F0375G, F0632G, and F0671G). Of the predecessor studies, 3 were open-label single-arm trials (F0206S, F0632G, and F0671G), 1 was investigator-sponsored (F0206S), and 
1 was multi-centre (F0671G). Study F0375G ( $n=8$ ) was a 27-month double-blind, placebocontrolled crossover trial including 6 months of mecasermin or placebo treatment, followed by a 3-month washout period, a 6-month crossover period, and a 12-month open-label extension study. Simple randomization was used to assign patients to the initial treatment group in Study F0375G. Because height velocity was an objective end point and the long-term height velocities in the other 4 studies were expected to be substantially greater than baseline and historical results in untreated children with SPIGFD, a randomized controlled group was deemed unnecessary in subsequent studies.

The purpose of this series of studies was to determine the safety and efficacy of long-term IGF-1 replacement therapy with mecasermin SC for the treatment of growth failure in children with SPIGFD. The linking of data from patients who participated in earlier trials allowed for each patient's data to be analyzed both individually and in aggregate with the rest of the treatment population. Many of the patients enrolled in Study 1419 had been continuously treated with mecasermin for many years and had transferred to another protocol when their first study ended. All patients enrolled in studies F0206S, F0375G, and F0632G were later enrolled in study F0671G. All patients (except 1) who enrolled in F0671G were later enrolled in Study 1419. The integrated study report includes results for patients enrolled in the 5 studies at 2 investigative sites in the US in conjunction with sites in 23 other countries worldwide. Two patients from Canada were enrolled.

Eligible patients in Study 1419 were those who had a height SDS of less than -2 for age and sex, a growth rate of less than the 50th percentile for age and sex for more than 6 months before study start, and an IGF-1 SDS of less than -2 for age and sex; were older than 18 months (no upper age limit was reported); and had open epiphyses. For those with growth hormone insensitivity syndrome (GHIS) and Laron syndrome, eligible patients needed to have a random or stimulated $\mathrm{GH}$ level of greater than $10 \mathrm{ng} / \mathrm{mL}$ and demonstrated failure to increase IGF- 1 by $50 \mathrm{ng} / \mathrm{mL}$ in response to exogenous GH during an IGF-1 generation test. For those with GHIS and GH gene deletion, eligibility required the presence of $\mathrm{GH}$ antibodies to exogenous $\mathrm{GH}$ with a binding capacity of greater than $10 \mathrm{mcg} / \mathrm{mL}$. Ineligible patients were those with active malignancy or any history of malignancy, growth failure due to other reasons, treatment with any corticosteroids or other medications that influence growth, and a clinically significant electrocardiogram abnormality or a history of a clinically significant cardiac arrhythmia.

Ninety-two patients were enrolled in Study 1419. The mean chronological age at baseline was 7.6 years (standard deviation $[S D]=4.3)$. The mean bone age was 3.8 years $(S D=2.8)$. More than half of the patients were male $(n=53 ; 58 \%)$ and the etiology of GHIS for most patients was Laron syndrome $(n=82 ; 89 \%)$. Most patients began treatment at pubertal stage $1(n=$ $79 ; 86 \%)$. Few $(n=9 ; 10 \%)$ had received prior IGF-1 therapy. Most patients (84\%) were White. All patients had severe short stature, with a mean height and height SDS of $88.5 \mathrm{~cm}(\mathrm{SD}=$ $20.7)$ and $-6.7(S D=1.9)$, respectively. The mean pre-treatment height velocity and height velocity SDS were $2.6 \mathrm{~cm}(S D=1.8)$ per year and $-3.2(S D=1.8)$, respectively. All but 1 patient $(\mathrm{n}=91$ of $92 ; 99 \%)$ had a pre-treatment height velocity SDS of -3 or lower. Patients started treatment at a mean body weight of $14.1(\mathrm{SD}=8.8) \mathrm{kg}$. The mean body mass index (BMI) and $\mathrm{BMI}$ score at baseline were $16.6 \mathrm{~kg} / \mathrm{m}^{2}(\mathrm{SD}=2.8)$ and $-0.2(\mathrm{SD}=1.2)$, respectively.

Patients received mecasermin $60 \mathrm{mcg} / \mathrm{kg}$ to $120 \mathrm{mcg} / \mathrm{kg}$ SC twice daily within 30 minutes of a meal. Naive-to-mecasermin patients generally started mecasermin at $60 \mathrm{mcg} / \mathrm{kg}$ to 80 $\mathrm{mcg} / \mathrm{kg}$ SC twice daily for 1 to 2 weeks and then increased to $120 \mathrm{mcg} / \mathrm{kg}$ SC twice daily as tolerated. The primary efficacy outcomes were height velocity, near-adult height, and 
estimated improvement in near-adult height. Secondary efficacy outcomes were height velocity SDS, height SDS, change in bone age relative to change in chronological age, and BMI SDS. Data on harms throughout treatment were also collected. The only comparator for efficacy outcomes was within-patient change from baseline, with the exception of estimated improvement in near-adult height, for which a historical cohort of patients with untreated Laron syndrome ${ }^{18}$ was used. The longest follow-up was 19 years.

\section{Efficacy Results}

All patients included in the primary efficacy analysis were naive to mecasermin and had been receiving treatment for at least 1 year $(n=75)$. Most commonly, patients received mecasermin $120 \mathrm{mcg} / \mathrm{kg}$ SC twice daily (356 patient-years or $69 \%$ of a total of 516 patientyears). Most of the rest of the exposure was at $80 \mathrm{mcg} / \mathrm{kg} \mathrm{SC}$ twice daily (50 patient-years or $10 \%$ of a total of 516 patient-years). A summary of the key results after the first year of treatment among naive-to-mecasermin patients are provided in Table 2.

\section{Height Velocity}

During year 1 of treatment, there was an increase in mean height velocity from $2.6 \mathrm{~cm}$ per year $(S D=1.7)$ to $8.0 \mathrm{~cm}$ per year $(S D=2.3)$. Height velocities for years 2 through 8 of treatment remained greater than baseline (i.e., $5.9 \mathrm{~cm}$ per year $[S D=1.7]$ in year 2 and $4.4 \mathrm{~cm}$ per year $[S D=1.5]$ in year 8 ). There was no correlation between age at the start of treatment and height velocity during the first year of treatment. The mean difference in year 1 height velocity was not statistically different in patients with $\mathrm{GH}$ gene deletion $(7.4 \mathrm{~cm}$ per year [SD = 3.6]; $n=7$ ) and patients with Laron syndrome phenotype ( $6.6 \mathrm{~cm}$ per year [SD $=3.8] ; n=72)$. There was no statistically significant difference in mean height velocity during the first year of treatment for those with antibodies $(7.9 \mathrm{~cm}$ per year [SD $=2.1])$ compared to those without antibodies $(7.1 \mathrm{~cm}$ per year [SD $=3.0])$. There was an observed association of dose on height velocity during the first year of treatment. The mean year 1 height velocity at a dosage of no more than $60 \mathrm{mcg} / \mathrm{kg} \mathrm{SC}$ twice daily was $6.0 \mathrm{~cm}$ per year (95\% confidence interval [Cl], 5.1 to 6.9) compared with $8.5 \mathrm{~cm}$ per year ( $95 \% \mathrm{Cl}, 7.8$ to 9.1 ) at a dosage of $120 \mathrm{mcg} / \mathrm{kg} \mathrm{SC}$ twice daily.

\section{Height Velocity Standard Deviation Score}

During year 1 of treatment, the mean height velocity SDS increased from $-3.4(S D=1.6)$ to $1.7(S D=2.8)$. The mean height velocity SDS for years 2 through 10 of treatment remained greater than baseline (i.e., -0.0 [SD $=1.7]$ during year 2 and 0.1 [SD $=0.6]$ during year 10). Results for patients who were naive to mecasermin treatment when they were enrolled in Study 1419 (i.e., excluding patients who had enrolled in any of the other 4 studies or were previously treated with Pharmacia mecasermin) were similar to those in the primary efficacy analysis (which included those who were naive to mecasermin at enrolment in any of the studies, including the predecessor studies and Study 1419 , but excluded those previously treated with Pharmacia mecasermin).

\section{Near-Adult Height and Estimated Improvement in Near-Adult Height}

Nineteen naive-to-mecasermin patients achieved near-adult height based on bone age criteria ( $\geq 16$ years for males and $\geq 14$ years for females). An additional 2 mecasermin-naive patients were considered by the investigators to have completed the intended course of treatment to near-adult height. The mean difference between the observed and expected increase in height (based on untreated patients with Laron syndrome, who achieved a mean final adult height of $124 \mathrm{~cm}$ [SD $=8.5]$ for males and $119 \mathrm{~cm}$ [SD = 8.5] for females, according to Laron et al. 
[2013] growth charts) ${ }^{18}$ was $13 \mathrm{~cm}(\mathrm{SD}=8$; range $=-0.5$ to 35$)$ after an average 11 years of treatment. The median final adult height was $137.6 \mathrm{~cm}$ (range $=112.0$ to 164.4).

\section{Height Standard Deviation Score}

During the first year of treatment, mean height SDS increased from $-6.9(S D=1.8)$ to -6.1 $(S D=1.8)$. The mean height SDS for years 2 through 14 of treatment remained greater than baseline (i.e., $-5.6[S D=1.7]$ in year 2 and $-4.3[S D=1.0]$ in year 14). Results for patients who were naive to mecasermin treatment when they were enrolled in Study 1419 were similar to those in the primary efficacy analysis.

\section{Health-Related Quality of Life}

Health-related quality of life (HRQoL) was not assessed in Study 1419 or its predecessors.

\section{Bone Age Relative to Chronological Age}

For patients with bone age measurements after at least 1 year of treatment $(n=56)$, bone age was delayed at baseline by a mean of 2.8 years $(S D=1.7)$ compared with chronological age (3.9 years [SD $=2.9]$ versus 6.7 years [SD $=3.8$, respectively). The change in bone age, for those with measurements after at least 1 year of treatment up to a maximum 17 years of treatment, exceeded the change in chronological age by a mean of 0.9 years $(S D=1.8)(+7.4$ years $[S D=3.7]$ versus +6.5 years $[S D=3.7]$, respectively).

\section{Body Mass Index Standard Deviation Score}

During the study there was a mean increase in BMI SDS from -0.3 (SD = 1.1) at baseline to $0.3(S D=1.4)$ when last evaluated, for a difference of $0.6(S D=1.3)$.

\section{Harms Results}

Seventy-six patients (83\%) had at least 1 adverse event (AE). The most reported AEs included metabolism and nutrition disorders $(n=48 ; 52 \%)$; general disorders and administration site conditions ( $n=42 ; 46 \%)$; infections and infestations $(n=41 ; 45 \%)$; respiratory, thoracic, and mediastinal disorders $(n=38 ; 41 \%)$; gastrointestinal disorders $(n=33 ; 36 \%)$; nervous system disorders ( $n=31 ; 34 \%)$; and musculoskeletal and connective tissue disorders ( $n=$ 29; $32 \%$ ). Eighteen patients (20\%) had at least 1 serious adverse event (SAE) that required hospitalization. No patient withdrew from the study due to an AE and no patient died during the study. The most frequently reported notable harms included hypoglycemia $(n=43 ; 47 \%)$; lipohypertrophy at the injection site $(n=32 ; 35 \%)$; tonsillar hypertrophy $(n=19 ; 21 \%)$; and adenoidal hypertrophy $(n=9 ; 10 \%)$.

\section{Critical Appraisal}

Study 1419 was a non-randomized, single-arm, open-label trial. Due to the rare and severe nature of SPIGFD, a randomized control group may not have been feasible or ethical. Due to the lack of a randomized control group, the findings are at high risk of confounding, and establishing a causal link between the treatment and the growth outcomes and harms is not possible. It is unclear how patients were selected for enrolment, so there is a potential for selection bias. The study may have been underpowered to detect statistically significant changes in outcomes, particularly at later time points when fewer patients remained in the study (e.g., after 8 years of treatment when fewer than 15 patients had measurements available for change in height velocity). There is an increased risk of type I error (i.e., false-positive conclusions) because no adjustments were made for multiple comparisons. For the estimated improvement in adult height, a historical control group of patients with untreated Laron syndrome was used. ${ }^{18}$ It is uncertain whether the final adult height in the 
Table 2: Summary of Key Results After 1 Year of Treatment in Study 1419 and Reports of Harms During Treatment

\begin{tabular}{|c|c|}
\hline \multicolumn{2}{|c|}{ Study 1419} \\
\hline \multicolumn{2}{|c|}{ Height velocity (cm per year), $\mathrm{n}=75^{\mathrm{a}}$} \\
\hline Baseline height velocity, mean (SD) & $2.6(1.7)$ \\
\hline Height velocity during year 1 , mean (SD) & $8.0(2.3)$ \\
\hline Change from baseline height velocity, mean (SD) & $5.4(2.6)$ \\
\hline$P$ value ${ }^{b}$ & $<0.0001$ \\
\hline \multicolumn{2}{|c|}{ Height velocity standard deviation score, $n=75^{a}$} \\
\hline Baseline height velocity SDS, mean (SD) & $-3.4(1.6)$ \\
\hline Height velocity SDS during year 1 , mean (SD) & $1.7(2.8)$ \\
\hline Change from baseline height velocity SD score, mean (SD) & $5.2(2.9)$ \\
\hline$P$ value ${ }^{b}$ & $<0.0001$ \\
\hline \multicolumn{2}{|c|}{ Near-adult height $(\mathrm{cm}), \mathrm{n}=19^{c}$} \\
\hline Final near-adult height, median & 137.6 \\
\hline Minimum to maximum & 112.0 to 164.4 \\
\hline Estimated improvement in adult height, mean (SD) & $13(8)$ \\
\hline Minimum to maximum & -0.5 to 35 \\
\hline \multicolumn{2}{|c|}{ Height SD score, $n=81^{a}$} \\
\hline Baseline height SDS, mean (SD) & $-6.9(1.8)$ \\
\hline Height SDS during year 1 , mean (SD) & $-6.1(1.8)$ \\
\hline Change from baseline height SDS, mean (SD) & $0.8(0.6)$ \\
\hline$P$ value ${ }^{b}$ & $<0.0001$ \\
\hline \multicolumn{2}{|c|}{ Bone age relative to chronological age (years), $n=56^{d}$} \\
\hline Baseline bone age, mean (SD) & $3.9(2.9)$ \\
\hline Baseline chronological age, mean (SD) & $6.7(3.8)$ \\
\hline Baseline bone age delay, mean (SD) & $2.8(1.7)$ \\
\hline Last bone age, mean (SD) & 11.3 (NA) \\
\hline Last chronological age, mean (SD) & $13.2(\mathrm{NA})$ \\
\hline Last bone age delay, mean (SD) & $1.9(\mathrm{NA})$ \\
\hline Change from baseline bone age, mean (SD) & $7.4(3.7)$ \\
\hline Change from baseline chronological age, mean (SD) & $6.5(3.7)$ \\
\hline $\begin{array}{l}\text { Difference of change in bone age and change in chronological } \\
\text { age, mean (SD) }\end{array}$ & $0.9(1.8)$ \\
\hline$P$ value ${ }^{b}$ & 0.0004 \\
\hline
\end{tabular}


Study 1419

\begin{tabular}{|l|c|}
\hline \multicolumn{2}{|c|}{ Body mass index standard deviation score, $\mathbf{n = 8 1}$} \\
\hline Baseline body mass index SDS, mean (SD) & $-0.3(1.1)$ \\
\hline Last body mass index SDS, mean (SD) & $0.3(1.4)$ \\
\hline Change from baseline body mass index SDS, mean (SD) & $0.6(1.3)$ \\
\hline P value $^{\mathrm{b}}$ & $<0.0001$ \\
\hline \multicolumn{2}{|c|}{ Harms, $\mathbf{n}=92$} \\
\hline Patients with $\geq 1$ AE, $\mathrm{n}(\%)$ & $76(83)$ \\
\hline Patients with $\geq 1$ SAE, $\mathrm{n}(\%)$ & $18(20)$ \\
\hline Patients who stopped treatment due to AEs, $\mathrm{n}(\%)$ & $0(0)$ \\
\hline Deaths, $\mathrm{n}$ (\%) & $0(0)$ \\
\hline Notable harms, $\mathrm{n}$ (\%) & \\
\hline Hypoglycemia & $43(47)$ \\
\hline Lipohypertrophy at the injection site & $32(35)$ \\
\hline Tonsillar hypertrophy & $19(21)$ \\
\hline Adenoidal hypertrophy & $9(10)$ \\
\hline Arthralgia & $8(9)$ \\
\hline Benign, malignant, and unspecified neoplasms (including cysts & $7(8)$ \\
\hline and polyps) & $7(8)$ \\
\hline Hypoglycemic seizure or convulsion & $6(7)$ \\
\hline Benign intracranial hypertension & $2(2)$ \\
\hline Myalgia & $2(2)$ \\
\hline Nephrolithiasis & \\
\hline
\end{tabular}

$\mathrm{AE}$ = adverse event; $\mathrm{NA}$ = not available; $\mathrm{SAE}$ = serious adverse event; $\mathrm{SD}$ = standard deviation; SDS = standard deviation score.

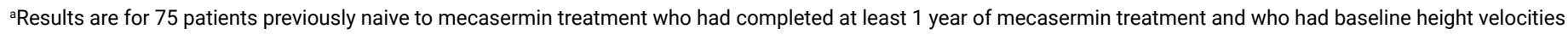

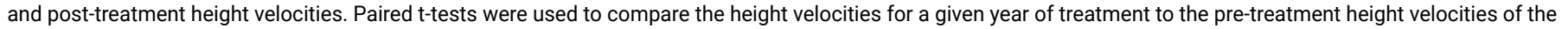
same patients completing that treatment year.

${ }^{\text {bP }}$ value was not adjusted for multiple comparisons.

${ }^{\mathrm{C}}$ Results are for 19 patients naive to mecasermin treatment who achieved near-adult height during the study. The comparator for estimated improvement in near-adult height is a historical cohort of patients with untreated Laron syndrome (Laron et al. [1993]). ${ }^{18}$

${ }^{\mathrm{d}}$ Results are for 56 patients naive to mecasermin treatment with a baseline bone age evaluation and a second bone age evaluation after at least one 1 year of treatment who were not receiving concomitant treatment with leuprolide.

Source: Clinical Study Report for Study $1419 .{ }^{15}$

historical cohort is representative of contemporary patients with SPIGFD. There is a risk that the estimated improvement in adult height could be biased due to differences in baseline characteristics of patients in Study 1419 compared to those studied by Laron et al. (1993). ${ }^{19}$ (Most notably, the etiology of SPIGFD in Study 1419 was not Laron syndrome in all patients and the patient population was multinational.) Further, there are no data to determine whether the final adult height in untreated patients has changed since the time of data collection by Laron et al. nearly 30 years ago. Because the trial was open-label, there is some risk that common subjective harms known to be associated with mecasermin could have been over-reported. A large proportion of patients (62\%) discontinued treatment early, many 
(33\%) of whom were lost to follow-up before attaining near-adult height. There is a high risk that the long-term efficacy and harms data could be biased due to missing outcomes for these patients.

Study 1419 included an international group of patients with SPIGFD, with eligibility criteria that allowed for patients with less-severe short stature than that described in the product monograph. ${ }^{12}$ Because only 1 patient had a baseline height SDS greater than -3 , based on height alone, the patient population is closely reflective of eligible Canadian patients. Based on the eligibility criteria, some patients without a genetic cause of SPIGFD may have been excluded and it is uncertain whether the results can be extrapolated to this group of patients. Although the condition affects males and females equally, there was an imbalance in the proportion of males and females enrolled in the study ( $58 \%$ were male). This is unlikely to strongly affect the generalizability of the findings because the treatment is expected to have an equivalent effect in males and females. Exposure to mecasermin is likely reflective of typical exposures for patients treated in the Canadian context. Twenty-one patients were treated with leuprolide to prolong the growth period in Study 1419. Although leuprolide is not approved for this indication in Canada, some physicians may choose to use leuprolide in conjunction with mecasermin, particularly among patients who are near an adult height or for whom bone age is rapidly increasing.

\section{Indirect Comparisons}

No indirect comparisons were identified or submitted by the sponsor.

\section{Other Relevant Evidence: The European Increlex Growth Forum Database Registry Description of Study}

The European Increlex Growth Forum Database (EU-IGFD) Registry ${ }^{20-22}$ is a descriptive, multicentre, observational, prospective, open-ended, noninterventional, post-authorization safety surveillance study of mecasermin. The primary objective was to collect long-term safety data on the use of mecasermin for the treatment of children with growth failure. Patients were eligible if they were beginning therapy with mecasermin for growth retardation or were previously treated with mecasermin prescribed by a participating qualified practitioner.

As of May 13, 2019, a total of 281 patients from 10 European countries were enrolled. Of these, 275 who had taken mecasermin at least once and completed at least 1 follow-up visit were included in the efficacy analysis. The mean chronological age at baseline was 9.5 years $(S D=4.1)$. Mean bone age was 8.6 years $(S D=3.5)$. More than half of patients were male $(n=177 ; 63 \%)$ and most did not have Laron syndrome $(n=238 ; 85 \%)$. Most patients began treatment at pubertal stage $1(n=225 ; 80 \%)$. Few $(n=24 ; 9 \%)$ had received prior IGF-1 therapy. About 1-quarter $(n=73 ; 26 \%)$ had received prior therapy with $\mathrm{GH}$. All patients had severe short stature, with a mean height and height SDS of $114.3 \mathrm{~cm}(\mathrm{SD}=21.4)$ and -3.8 $(S D=1.3)$, respectively. Mean pre-treatment height velocity was $4.7 \mathrm{~cm}(S D=1.7)$ per year. The mean body weight and body weight SDS at baseline were $22.0 \mathrm{~kg}(\mathrm{SD}=9.8)$ and -3.3 $(S D=1.4)$, respectively. The mean BMI and BMI SDS at baseline were $16.1 \mathrm{~kg} / \mathrm{m}^{2}(S D=2.9)$ and $-0.7(S D=1.4)$, respectively.

All patients received mecasermin at a recommended starting dosage of $0.04 \mathrm{mg} / \mathrm{kg} \mathrm{SC}$ twice daily and maximum dosage of $0.12 \mathrm{mg} / \mathrm{kg}$ SC twice daily. The recommended dose was individualized for each patient based on treatment response and tolerance. 


\section{Efficacy Results}

Efficacy outcomes were evaluated for the whole registry population $(n=275)$, as well as for prepubertal patients naive to mecasermin $(n=162)$ and for pubertal or previously treated patients $(n=109)$. A summary of the key results after the first year of treatment among prepubertal naive-to-mecasermin patients is provided in Table 3.

\section{Height}

The mean height at baseline among prepubertal naive-to-mecasermin patients was 107.2 $\mathrm{cm}(\mathrm{SD}=20.4)$. The mean change from baseline after 1 year of treatment was $+7.2 \mathrm{~cm}$ $(S D=2.2)$. The mean changes in height from baseline in years 2 through 5 of treatment were $+13.8 \mathrm{~cm}(\mathrm{SD}=3.2),+19.5 \mathrm{~cm}(\mathrm{SD}=4.0),+25.0 \mathrm{~cm}(\mathrm{SD}=4.2)$, and $+30.9 \mathrm{~cm}(\mathrm{SD}=4.7)$, respectively.

The mean height SDS at baseline among prepubertal naive-to-mecasermin patients was $-3.8(S D=1.4)$. The mean change from baseline after 1 year of treatment was $+0.4(S D=$ 0.4). There was an association between age and height SDS during the first year of treatment $(P=0.024)$. The mean changes in height SDS in years 2 through 5 of treatment were +0.7 $(S D=0.6),+0.9(S D=0.6),+1.1(S D=0.6)$, and $+1.2(S D=0.8)$, respectively. Among patients with Laron syndrome, the mean height SDS at baseline was $-5.0(S D=1.75)$. The mean change from baseline after 1 year of treatment was $+0.9(S D=0.8)$. Among patients without Laron syndrome, the mean height SDS at baseline was $-3.6(S D=1.1)$. The mean change from baseline after 1 year of treatment was $+0.3(S D=0.4)$.

The mean height velocity at baseline among prepubertal naive-to-mecasermin patients was $4.8 \mathrm{~cm}$ per year $(S D=1.8)$. The mean change from baseline after 1 year of treatment was $+2.5 \mathrm{~cm}$ per year $(S D=2.5)$. The mean changes in height velocity in years 2 through 5 of treatment were $+1.8 \mathrm{~cm}$ per year $(S D=2.3),+1.1 \mathrm{~cm}$ per year $(S D=2.6),+0.8 \mathrm{~cm}$ per year $(S D=2.2)$, and $+0.8 \mathrm{~cm}$ per year $(S D=1.8)$, respectively. In patients concomitantly treated with $\mathrm{GH}$, mean height velocity at baseline was $4.6 \mathrm{~cm}$ per year $(S D=1.8)$. The mean changes from baseline after 1 and 2 years of treatment were $+1.2 \mathrm{~cm}$ per year $(S D=3.0)$ and +0.7 $\mathrm{cm}$ per year $(S D=2.9)$, respectively. For patients with Laron syndrome, mean height velocity at baseline was $4.8 \mathrm{~cm}$ per year ( $S D=1.3$ ). The mean change from baseline after 1 year of treatment was $+1.3 \mathrm{~cm}$ per year $(S D=2.5)$. The mean changes in height velocity from baseline in years 2 through 4 of treatment were $+0.7 \mathrm{~cm}$ per year $(S D=2.5),-1.9 \mathrm{~cm}$ per year $(S D=3.0)$, and $-0.3 \mathrm{~cm}$ per year $(S D=2.7)$, respectively. In patients without Laron syndrome, mean height velocity at baseline was $4.7 \mathrm{~cm}$ per year $(S D=1.8)$. The mean change from baseline after 1 year of treatment was $+2.2 \mathrm{~cm}$ per year $(S D=2.6)$. The mean changes in height SDS from baseline in years 2 through 5 of treatment were $+1.5 \mathrm{~cm}$ per year $(\mathrm{SD}=2.3)$, $+1.3 \mathrm{~cm}$ per year $(\mathrm{SD}=2.5),+0.7 \mathrm{~cm}$ per year $(\mathrm{SD}=2.2)$, and $+0.2 \mathrm{~cm}$ per year $(\mathrm{SD}=1.8)$, respectively.

The mean final adult height and final adult height SDS among prepubertal naive-tomecasermin patients were $158.6 \mathrm{~cm}(S D=12.6)$ and $-2.3(S D=1.2)$, respectively. There was an association between age at baseline $(P<0.001)$, predicted adult height $(P<0.001)$, height SDS at baseline $(P=0.003)$, and final adult height (i.e., those who begin treatment at a younger age with a higher height SDS may achieve a higher final adult height). There was an association between predicted adult height $(P<0.001)$ and height SDS at baseline $(P=0.016)$ and final adult height SDS. 
Health-Related Quality of Life

HRQoL was measured in 47 patients from France and/or their parents; however, baseline and follow-up data were only available for 2 patients and could not be summarized in this report.

\section{Bone Age}

The mean bone age at baseline among prepubertal naive-to-mecasermin patients was 7.3 years $(S D=3.1)$ years. The mean change from baseline after 1 year of treatment was +1.1 years $(S D=0.5)$ years. The mean changes in bone age in years 2 and 3 of treatment were +2.3 years $(S D=0.6)$ years and +3.3 years $(S D=0.6)$, respectively. The difference between bone age and chronological age at baseline was -1.9 years $(S D=1.0)$. The mean change from baseline after 1 year of treatment was +0.1 years $(S D=0.6)$. The mean changes in the difference between bone age and chronological age in years 2 and 3 of treatment were +0.2 years $(S D=0.6)$ and +0.2 years $(S D=0.6)$, respectively.

\section{Weight and Weight Standard Deviation Score}

The mean weight at baseline among prepubertal naive-to-mecasermin patients was $18.4 \mathrm{~kg}$ $(\mathrm{SD}=7.3)$ and changed by a mean of $+3.3 \mathrm{~kg}(\mathrm{SD}=1.8)$ during the first year of treatment. The mean changes in weight in years 2 through 5 of treatment were $+6.6 \mathrm{~kg}(\mathrm{SD}=3.6),+9.9 \mathrm{~kg}$ $(S D=5.0),+12.0 \mathrm{~kg}(S D=5.4)$, and $+15.6 \mathrm{~kg}(S D=6.1)$, respectively. The mean weight SDS at baseline was $-3.4(S D=1.4)$ and changed by a mean of $+0.5(S D=0.7)$ during the first year of treatment. The mean changes in weight SDS in years 2 through 5 of treatment were +0.8 $(S D=1.0),+1.0(S D=1.1),+1.3(S D=1.3)$, and + 1.6 $(S D=1.4)$, respectively.

\section{Body Mass Index Standard Deviation Score}

The mean BMI SDS at baseline among prepubertal naive-to-mecasermin patients was -0.8 $(S D=1.3)$. The mean change from baseline after 1 year of treatment was $+0.2(S D=0.7)$. The mean changes in BMI SDS in years 2 through 5 of treatment were $+0.3(S D=0.7),+0.4 S D=$ $(0.8),+0.4(S D=1.0)$, and $+0.6(S D=1.1)$, respectively.

\section{Harms Results}

Harms During Treatment

A total of 185 patients (67\%) had at least 1 AE during treatment and $59(21 \%)$ had at least 1 SAE. Fifteen patients withdrew from the study due to AEs and 2 patients ( $1 \%$ ) died (1 patient from myelodysplastic syndrome and 1 due to complications of a bone marrow transplant).

The most frequently reported notable harms included hypoglycemia $(n=68 ; 25 \%)$, lipohypertrophy at the injection site $(n=33 ; 12 \%)$ and tonsillar hypertrophy $(n=25 ; 9 \%)$. Myalgia ( $n=4 ; 1 \%)$; benign, malignant, and unspecified neoplasms (including cysts and polyps) $(n=2 ; 1 \%)$; and intracranial hypertension $(n=1 ; 0.4 \%)$ were reported less frequently.

\section{Harms Post-Treatment}

During the post-treatment period, 39 AEs were reported in 21 patients (13\%). Seventeen SAEs were reported.

During the long-term safety period, 5 patients (29\%) experienced 6 AEs (tonsilitis, cyclic vomiting syndrome, hearing loss, tonsillar hypertrophy, decreased thyroxine free, and decreased vitamin D). Two SAEs (tonsillitis and cyclic vomiting syndrome) were reported.

No patient died during the post-treatment or long-term safety periods. 
Table 3: Summary of Key Results After 1 Year of Treatment in the EU-IGFD Registry and Reports of Harms During Treatment ${ }^{\mathrm{a}}$

\begin{tabular}{|c|c|}
\hline Result & EU-IGFD Registry \\
\hline \multicolumn{2}{|c|}{ Height $(\mathrm{cm}), \mathrm{n}=120$} \\
\hline Baseline height, mean (SD) & $107.2(20.4)$ \\
\hline Height after 1 year, mean (SD) & $113.8(21.2)$ \\
\hline Change from baseline height, mean (SD) & $7.2(2.2)$ \\
\hline \multicolumn{2}{|c|}{ Height standard deviation score, $n=120$} \\
\hline Baseline height SDS, mean (SD) & $-3.8(1.4)$ \\
\hline Height SDS after 1 year, mean (SD) & $-3.4(1.4)$ \\
\hline Change from baseline height SDS, mean (SD) & $0.4(0.4)$ \\
\hline \multicolumn{2}{|c|}{ Height velocity (cm per year), $n=118$} \\
\hline Baseline height velocity, mean (SD) & $4.8(1.8)$ \\
\hline Height velocity after 1 year, mean (SD) & $7.2(2.1)$ \\
\hline Change from baseline height velocity, mean (SD) & $2.5(2.5)$ \\
\hline \multicolumn{2}{|c|}{ Final adult height $(\mathrm{cm})$ and adult height standard deviation score, $\mathrm{n}=32$} \\
\hline Final adult height, mean (SD) & $155.1(13.2)$ \\
\hline Minimum to maximum & 131 to 182 \\
\hline Final adult height SDS, mean (SD) & $-2.3(1.2)$ \\
\hline Minimum to maximum & -7 to 0 \\
\hline \multicolumn{2}{|c|}{ Bone age (years), $n=10$} \\
\hline Baseline bone age, mean (SD) & $7.3(3.1)$ \\
\hline Bone age after 1 year, mean (SD) & $7.3(3.3)$ \\
\hline Change from baseline bone age, mean (SD) & $1.1(0.5)$ \\
\hline \multicolumn{2}{|c|}{ Difference between bone age and chronological age (years), $n=10$} \\
\hline $\begin{array}{l}\text { Baseline difference between bone age and chronological age, } \\
\text { mean (SD) }\end{array}$ & $-1.9(1.0)$ \\
\hline $\begin{array}{l}\text { Difference between bone age and chronological age after } 1 \text { year, } \\
\text { mean (SD) }\end{array}$ & $-1.8(1.2)$ \\
\hline $\begin{array}{l}\text { Change from baseline difference between bone age and } \\
\text { chronological age, mean (SD) }\end{array}$ & $0.1(0.6)$ \\
\hline \multicolumn{2}{|c|}{ Weight (kg), $n=121$} \\
\hline Baseline weight, mean (SD) & $18.4(7.3)$ \\
\hline Weight after 1 year, mean (SD) & $21.6(8.8)$ \\
\hline Change from baseline weight, mean (SD) & $3.3(1.8)$ \\
\hline
\end{tabular}




\begin{tabular}{|c|c|}
\hline \multicolumn{2}{|c|}{ Weight standard deviation score, $n=121$} \\
\hline Baseline weight SDS, mean (SD) & $-3.4(1.4)$ \\
\hline Weight SDS after 1 year, mean (SD) & $-2.9(1.6)$ \\
\hline Change from baseline weight SDS, mean (SD) & $0.5(0.7)$ \\
\hline \multicolumn{2}{|c|}{ BMI standard deviation score, $n=106$} \\
\hline Baseline BMI SDS, mean (SD) & $-0.8(1.3)$ \\
\hline BMI SDS after 1 year, mean (SD) & $-0.5(1.4)$ \\
\hline Change from baseline BMI SDS, mean (SD) & $0.2(0.7)$ \\
\hline \multicolumn{2}{|c|}{ Harms during treatment, $n=277^{b}$} \\
\hline Patients with $\geq 1 \mathrm{AE}, \mathrm{n}(\%)$ & $185(67)$ \\
\hline Patients with $\geq 1 \mathrm{SAE}, \mathrm{n}(\%)$ & $59(21)$ \\
\hline Patients who stopped treatment due to AEs, n (\%) & $15(5)$ \\
\hline Deaths, n (\%) & $2(1)$ \\
\hline \multicolumn{2}{|l|}{ Notable harms, n (\%) } \\
\hline Hypoglycemia & $68(25)$ \\
\hline Lipohypertrophy at the injection site & $33(12)$ \\
\hline Tonsillar hypertrophy & $25(9)$ \\
\hline Myalgia & $4(1)$ \\
\hline $\begin{array}{l}\text { Benign, malignant, and unspecified neoplasms (including cysts } \\
\text { and polyps) }\end{array}$ & $2(1)$ \\
\hline Intracranial hypertension & $1(0.4)$ \\
\hline
\end{tabular}

$\mathrm{AE}=$ adverse event; $\mathrm{BMI}=$ body mass index; EU-IGFD = European Increlex Growth Forum Database; $\mathrm{SAE}=$ serious adverse event; $\mathrm{SD}=$ standard deviation; $\mathrm{SDS}=$ standard deviation score.

${ }^{a}$ Changes from baseline are for paired analyses among patients who had baseline data and data available after 1 year of treatment, as per the reported sample size. The baseline values provided are for all patients measured at baseline, which could differ from those measured after 1 year.

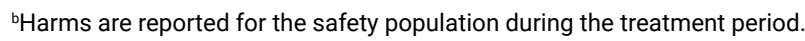

Source: Clinical Study Report for EU-IGFD Registry. ${ }^{20}$

\section{Critical Appraisal}

The EU-IGFD Registry provides real-world evidence of growth and safety outcomes among patients treated with mecasermin for SPIGFD. Due to the lack of a randomized control group, the findings are at high risk of confounding, and establishing a causal link between the treatment and efficacy and harms is not possible. There is an increased risk of type I error because there was no adjustment for multiple comparisons. It is unclear whether patients were consecutively enrolled, so there is a potential for selection bias. It is unclear whether the study was powered to detect statistically significant changes in growth outcomes (although these were not tested statistically). With respect to the analysis of harms, the sample size was not large enough to observe side effects with a true incidence of less than or equal to 1 per 100 . Only 17 patients were eligible for the long-term safety analysis. It is possible that common subjective harms known to be associated with mecasermin could have been over-reported, as patients and their treating clinicians knew of the treatment received and 
of their participation in a registry study. A large proportion of enrolled patients $(n=76 ; 27 \%)$ discontinued the study early for reasons other than completing the course of treatment or achieving final adult height. No data were collected after withdrawal. Additionally, baseline data were missing for a large proportion of enrolled patients across all outcomes. No imputations or other statistical methods were used to account for missing data and there is a high risk that efficacy and harms data could be biased due to missing data. Sixty-nine patients ( $25 \%$ of those enrolled) were affected by a mecasermin shortage over the course of the study, resulting in a dosage decrease or dose interruption. These decreases and interruptions could have attenuated the growth outcomes and harms observed.

The EU-IGFD Registry included an international group of patients treated with mecasermin. There were no inclusion criteria specific to Health Canada's approved indication for mecasermin (e.g., specifications for height, basal IGF-1 level, GH sufficiency, and exclusion of secondary forms of IGF-1 deficiency). ${ }^{12}$ However, the European Medicines Agency's therapeutic indication for mecasermin is identical to Health Canada's. ${ }^{12,23}$ Only $15 \%$ of patients were diagnosed with Laron syndrome (in contrast to $89 \%$ of those enrolled in Study 1419), which is the most common cause of SPIGFD. ${ }^{7}$ The mean height SDS was substantially short, on average, at baseline (mean of -3.8 [SD $=13]$ ). There was a large range of height SDSs at baseline (-9.4 to -1.3) and not all patients met the height SDS criterion in the product monograph (i.e., a height SDS of no more than -3 ). ${ }^{12}$ Height velocity ranged from 0.5 to $10.6 \mathrm{~cm}$ per year at baseline. Sixty-three percent of patients were male and the median chronological age at baseline was 9.6 years (range $=0.4$ to 19.1). These patients would be eligible for treatment according to the product monograph based on age, ${ }^{12}$ but are likely older than the optimal start time of treatment. Although SPIGFD affects males and females equally, the imbalance in the proportion of males and females enrolled is unlikely to affect the generalizability of the findings because the treatment is expected to have an equivalent effect on males and females. The exposure to mecasermin in the EU-IGFD Registry is likely reflective of typical exposures for patients treated in a Canadian context. Concomitant medications taken by patients during the study were similar to those that would be expected in Canadian clinical practice.

\section{Other Relevant Evidence: The Polish Study on Increlex Description of Study}

The Polish Study on Increlex ${ }^{24}$ was a single-arm trial that investigated efficacy and harms for patients with SPIGFD treated with mecasermin during the first 3 years it was covered by a therapeutic program in Poland. Patients were eligible if they were diagnosed with SPIGFD according to Polish criteria.

The study enrolled 27 patients, including $22(81 \%)$ males and 5 (19\%) females. The mean chronological age at baseline was 10.1 (range $=2.8$ to 16.2$)$ years. Nearly all $(n=25 ; 93 \%)$ patients were prepubescent. The mean height SDS and height velocity at baseline were -3.5 (range $=-6.5$ to -2.3 ) and $4.6 \mathrm{~cm}$ (range $=0.9$ to 7.5 ) per year, respectively. The mean weight SDS at baseline was -3.1 (range $=-5.8$ to -1.2 ). The mean BMI SDS at baseline was $-1.8(\mathrm{SD}=1.3)$.

All patients received mecasermin. The initial dose was $40 \mathrm{mcg} / \mathrm{kg} \mathrm{SC}$ twice daily and was increased over time. The maximum dose was $120 \mathrm{mcg} / \mathrm{kg} \mathrm{SC}$ twice daily. There was no control group and the only comparator was within-patient change from baseline. 


\section{Efficacy Results}

Growth outcomes were measured every 3 months up to 36 months (3 years) of treatment for 25 patients who completed the study.

\section{Height}

The results for height outcomes were consistent with those from the pivotal trial. There was an increase in the mean height SDS and height velocity during the first 3 years of treatment, with the greatest apparent increase in height velocity during the first year of treatment.

\section{Health-Related Quality of Life}

The Polish Study on Increlex did not measure HRQoL.

\section{Skeletal Maturation}

Skeletal maturation (e.g., bone age, bone age SDS) was measured in the Polish Study on Increlex, but the findings were not included in the published report.

\section{Body Mass}

The results for BMI SDS, which increased during the first 3 years of treatment, were consistent with those of the pivotal trial.

\section{Harms Results}

Eight (30\%) patients reported AEs during the study. The seriousness of AEs was not reported. Two patients (7\%) discontinued the study due to AEs. No patient died during the study. Regarding notable harms, 2 patients (7\%) reported hypoglycemia during the sixth month of treatment. Two patients (7\%) had hyperlipodystrophy at the injection site. One patient (4\%) developed hypertrophy of the lymphatic tissue of the pharyngeal tonsils.

\section{Critical Appraisal}

The Polish Study on Increlex was a single-arm trial that investigated growth outcomes during the first 3 years of treatment. Considering the rare and severe nature of SPIGFD, a randomized control group may not have been feasible or ethical. Due to the lack of randomized control group, the findings are at high risk of confounding, and establishing a causal link between the treatment and the growth outcomes and harms is not possible. There is an increased risk of type I error because no adjustments were made for multiple comparisons. It is possible that common subjective harms known to be associated with mecasermin could have been over-reported, as patients and their treating clinicians knew of the treatment received and of their participation in a trial. The study enrolled the first 27 children in Poland treated with mecasermin for SPIGFD and it does not appear that there is a substantial risk of selection bias. The power of the study is unclear; however, the results of all Mann-Whitney $U$ tests to investigate changes from baseline were statistically significant. It is not clear which growth references were used to calculate SDSs. For this reason, the validity of the outcomes based on SDSs is uncertain. There was no published protocol for the Polish Study on Increlex. The risk of bias due to selective reporting is high, primarily because some clinically important outcomes were measured but not reported.

The eligibility criteria were aligned with the product monograph ${ }^{12}$ and all patients underwent an IGF-1 generation test to assess for insensitivity to GH. The mean age at baseline was 10.1 years (range $=2.8$ to 16.2 ) and bone age was not reported. The majority of patients $(81 \%)$ were male. According to the product monograph, these patients would be eligible 
for treatment based on age ${ }^{12}$ but are likely older than the optimal start time of treatment. Although SPIGFD affects males and females equally, the imbalance in the proportion of males and females enrolled is unlikely to affect the generalizability of the findings because the treatment is expected to have an equivalent effect on males and females. The exposure to mecasermin in the Polish Study on Increlex is likely reflective of typical exposures for patients who would be treated in the Canadian context.

\section{Conclusions}

One phase III, multi-centre, single-arm, open-label trial with linked data from 4 predecessor studies was included. Two other studies provided additional relevant evidence: 1 registry study of European patients and 1 single-arm trial in Poland. Due to the rare and severe nature of SPIGFD, a randomized control group was not feasible or ethical in the included trials, making it impossible to infer a causal relationship between mecasermin treatment and growth and harms outcomes. Nevertheless, data from the pivotal trial (Study 1419) appear to demonstrate a clear response to mecasermin therapy for most patients, in the form of a marked improvement in 1-year height velocity that is not typical of untreated patients with SPIGFD. ${ }^{18}$ These findings are corroborated by those from smaller, shorter-term randomized trials of rhIGF-1 therapy for patients with growth retardation. 25,26

It appears that mecasermin treatment may improve final adult height in patients with SPIGFD compared with untreated patients with Laron syndrome. It is uncertain whether the historical control group used to support this conclusion is representative of contemporary untreated patients with SPIGFD. Of note, data from the pivotal trial showed that, during treatment, bone age advanced more quickly than did chronological age while BMI SDSs increased. The implications of these changes need to be considered when evaluating the potential benefits of mecasermin; however, because the analyses were not controlled for multiple comparisons, there is an increased risk of type I error, and conclusions for these outcomes are limited. None of the studies provided usable data on HRQoL or on the effects of mecasermin on other known health consequences of SPIGFD (e.g., metabolic abnormalities and muscle weakness).

Harms were commonly reported across all studies, but serious harms were less frequent and deaths were rare. Few patients withdrew from any of the studies due to AEs. Among the most reported AEs were hypoglycemia, lipohypertrophy at the injection site, and tonsillar hypertrophy. For the most part, these could be managed through careful monitoring and dosing changes or interruptions, and they rarely resulted in discontinuation of treatment. Other notable harms (e.g., benign or malignant neoplasia) were less frequent. Due to the challenges inherent in the identification of patients who might benefit most from mecasermin treatment, it may be helpful to engage a panel of clinical experts to inform treatment decisions, at least when a diagnosis cannot be confirmed. This may not be feasible as few Canadian clinicians have direct experience with the diagnosis and treatment of SPIGFD; however, physicians with expertise in managing pediatric endocrine growth disorders may also be qualified to contribute. A Canadian registry to monitor benefits and long-term harms in patients treated with mecasermin may be useful. 


\section{Introduction}

\section{Disease Background}

Severe primary insulin-like growth factor 1 deficiency is defined by a height SDS or no more than -3.0; basal IGF-1 levels below the 2.5th percentile for age and sex; GH sufficiency; and the exclusion of secondary forms of IGF-1 deficiency, including malnutrition, hypothyroidism, or chronic treatment with pharmacologic doses of anti-inflammatory steroids. ${ }^{1}$ It includes patients with mutations in the GHR gene, post-GHR signalling pathway, and IGF-1 gene defects. ${ }^{2,3}$ Case of SPIGFD, which is considered an orphan disease in the US and Europe, are extremely rare. ${ }^{1,27}$ In Canada, it is estimated that approximately 4.88 cases of SPIGFD are diagnosed each year, or 1 case in every 77,000 births (a prevalence of $0.0013 \%){ }^{4-6}$ Orphanet estimated the global prevalence of IGF-1 deficiency is less than 1 in 1,000,000 (<0.0001\%), with SPIGFD even rarer. ${ }^{28}$

Inadequate IGF-1 production despite sufficient secretion of GH is a key feature of SPIGFD. The secretion of $\mathrm{GH}$ and the functional integrity of the IGF system are essential for normal linear growth and its associated metabolic processes in children. Although the phenotypic and biochemical features of various genetic defects leading to SPIGFD may differ, all are characterized by severe growth failure (extreme short stature) ${ }^{29}$ Laron syndrome is the most common known cause of SPIGFD. ${ }^{7}$ The clinical features of untreated Laron syndrome, which is the result of GHR mutations, include severe post-natal growth failure (despite normal prenatal growth), short limb length (relative to trunk length), characteristic facial features (saddle nose and small forehead), delayed skeletal maturation and sexual development, small genitalia and testes, osteopenia, muscle weakness, obesity, and metabolic abnormalities such as hyperlipidemia, hypoglycemia, insulin resistance..$^{8-11}$

The diagnosis of SPIGFD requires the expertise of a pediatric endocrinologist ${ }^{9}$ and involves distinguishing between children with hormonal versus non-pathologic (e.g., familial short stature) and nonhormonal (e.g., Crohn disease) causes of short stature or growth failure. ${ }^{2}$ A full medical and nutritional history and laboratory screening studies (e.g., markers of liver, kidney, and thyroid function; celiac disease screen) are a first step. Serum IGF-1 and insulin-like growth factor binding protein 3 (IGFBP-3) measurements are also part of the initial evaluation. 930 Among those with low serum IGF-1, testing of GH stimulation should be done, as normal GH secretion concomitant with low IGF-1 suggests primary IGF-1 deficiency. 9,30 Although the diagnosis of SPIGFD can be made on the basis of a strong clinical phenotype and compatible biochemical testing, it can also be supported by molecular genetic testing for mutations in the GHR or other defects. ${ }^{2,30}$

Although SPIGFD is not considered a life-limiting disease, children with short stature may experience poorer HRQoL compared with children of normal (or average) stature, and their parents or caregivers may experience greater caregiver burden. ${ }^{31}$ There is evidence that the negative impact of short stature on HRQoL in children may persist into adulthood; however, research into the relationship between stature and quality of life in adults has been inconclusive. ${ }^{31}$ Due to the lack of research specifically involving patients with SPIGFD (as opposed to other causes of short stature), ${ }^{31}$ the extent to which living with SPIGFD affects the HRQoL of patients and their caregivers is uncertain. Studies in children with short stature due to other causes show that HRQoL tends to be worse in cases of more severe short stature and can improve with increases in height associated with appropriate treatment (e.g., $\mathrm{GH}$ therapy). ${ }^{31}$ 


\section{Standards of Therapy}

Physicians with expertise in managing pediatric endocrine disorders should be involved in the diagnosis and management of SPIGFD..$^{14}$ In Canada and globally, mecasermin is currently the only approved therapy for the long-term treatment of growth failure in children with SPIGFD. Upon diagnosis, the standard of treatment is administration of mecasermin as soon as possible. To maximize adult height, mecasermin is administered over several years. Compliance with dosing and administration regimens are important to achieving optimal outcomes and ensuring safety. ${ }^{9}$ Mecasermin should not be used to promote growth in patients with closed epiphyses,; those who are hypersensitive to the drug or to any ingredient in the formulation, those with active or suspect neoplasia or any condition that increases the risk of benign or malignant neoplasia, or premature infants or neonates. ${ }^{12}$

Guidelines from the Pediatric Endocrine Society ${ }^{14}$ recommend the use of IGF-1 therapy to increase height in children and adolescents with SPIGFD. A trial of GH therapy before starting IGF-1 treatment is recommended in patients with unexplained IGF-1 deficiency. ${ }^{14} \mathrm{~A}$ trial of $\mathrm{GH}$ is not required in patients who have hormone-signalling defects that are known to be unresponsive to $\mathrm{GH}$ treatment. ${ }^{14}$ The recommended dose for the treatment of SPIGFD, according to the guidelines, is $80 \mathrm{mcg} / \mathrm{kg}$ to $120 \mathrm{mcg} / \mathrm{kg}$ via SC injection twice daily, which is within the dosing recommendation in the product monograph. ${ }^{12}$ There is no strong evidence supporting the superiority of 1 dose over another. In some countries a dosage of $150 \mathrm{mcg} / \mathrm{kg}$ to $180 \mathrm{mcg} / \mathrm{kg}$ SC once daily is used.

According to the clinical expert consulted by CADTH for the purpose of this review, for patients with Laron syndrome, those with $\mathrm{GH}$ antibodies, and those with a clinically diagnosed IGF-1 deficiency and undetectable GHBP, there is no other treatment option aside from mecasermin. The main goal of treatment is to improve height velocity and final adult height. Presently, tests for GH antibodies and a GH-IGF-1 axis genetic panel are not always easily accessible. In addition, the IGF-1 generation test following short-term GH administration is poorly standardized and has pitfalls, and not all genetic causes of SPIGFD are known. ${ }^{14}$ Consequently, idiopathic short stature is presently treated with $\mathrm{GH}$ when no cause is found. In the few patients with true Laron syndrome, clear biochemical evidence of $\mathrm{GH}$ resistance, or $\mathrm{GH}$ antibodies, treatment with $\mathrm{GH}$ is not indicated. These patients are not expected to respond to high doses of $\mathrm{GH}$ and the treatment would then be discontinued due to poor response, without other alternatives.

It is important to clarify a diagnosis to prevent the inappropriate use of mecasermin, as patients with GH deficiency will also likely respond to mecasermin, and IGF-1 can be affected by a variety of issues (e.g., chronic disease and poor nutrition). The guidelines state that the diagnosis of SPIGFD should be based on a combination of factors that fall into 4 stages: (1) screening for auxological parameters (e.g., poor growth and other characteristics features) and low IGF-1 concentration; (2) exclusion of nonhormonal causes of IGF-1 deficiency; (3) very low or undetectable levels of GHBP, which suggests Laron syndrome; and (4) IGF-1 generation testing and genetic testing. ${ }^{14}$ Patients most likely to benefit from mecasermin treatment include those with SPIGFD (due to a genetic defect of the GH-IGF-1 pathway, biochemical evidence of inappropriate $\mathrm{GH}$ receptor, or $\mathrm{GH}$ antibodies) with a predicted adult height that falls short of their potential. Left untreated, a patient with Laron syndrome has a final expected height of $120 \mathrm{~cm}$ to $130 \mathrm{~cm} \cdot{ }^{18}$ Final height is improved with treatment but remains well below normal, and the response is not as good as that with $\mathrm{GH}$ in $\mathrm{GH}$-deficient patients. The earlier treatment with mecasermin begins, the better the results. 


\section{Drug}

Mecasermin $10 \mathrm{mg} / \mathrm{mL}$ is indicated for children and adolescents from 2 to 18 years for the treatment of growth failure associated with SPIGFD. ${ }^{12}$ A Notice of Compliance for mecasermin was issued by Health Canada on December 17, 2020. ${ }^{32}$ The requested reimbursement criteria from the sponsor is similar to the Health Canada indication. Mecasermin has not previously been reviewed by CADTH for any indication. The drug information provided in the following section is consistent with the product monograph. ${ }^{12}$

Mecasermin contains rhIGF-1, which is produced by recombinant DNA technology. It is identical to the naturally occurring human peptide, IGF-1, which is the principal mediator of statural growth in humans. In target tissues, the IGF-1 receptor, which is homologous to the insulin receptor, is activated by IGF-1, resulting in intracellular signalling that stimulates multiple processes that lead to statural growth. The metabolic actions of IGF-1 are directed, in part, at stimulating the uptake of glucose, fatty acids, and amino acids such that metabolism supports growing tissues, and at suppressing hepatic glucose production, stimulating peripheral glucose utilization, and inhibiting insulin secretion. ${ }^{12}$

Mecasermin is supplied in a $5 \mathrm{~mL}$ multi-dose vial, with each vial containing $4 \mathrm{~mL}(40 \mathrm{mg})$ of solution. The recommended starting dosage is $0.04 \mathrm{mg} / \mathrm{kg}$ to $0.08 \mathrm{mg} / \mathrm{kg}$ (40 mcg/ $\mathrm{kg}$ to $80 \mathrm{mcg} / \mathrm{kg}$ ) SC twice daily. The injection should be administered using sterile disposable syringes and needles shortly before or after a meal or snack. The injection sites should be rotated with each injection to help prevent lipohypertrophy. The dose should be tailored to each patient and adjusted based on tolerability and body weight. If well tolerated for at least 1 week, the dose may be increased by 0.04 mg/kg SC twice daily to a maximum of 0.12 mg/ $\mathrm{kg}$ SC twice daily. If the dose is not tolerated, treatment with a lower dose can be considered. If hypoglycemia occurs with recommended doses despite adequate food intake, the dose should be reduced. Treatment success should be evaluated based on height velocities. ${ }^{12}$

\section{Stakeholder Perspectives}

\section{Patient Group Input}

This section was prepared by CADTH staff based on the input provided by patient groups.

\section{About the Patient Group and Information Gathered}

CADTH received 1 patient group submission for the review of mecasermin from ICOSEP, which regularly communicates with patient organization leaders and medical professionals across the world. The organization focuses on endocrine health care issues and works toward the goals of uniting medical societies and patient organizations as well as facilitating education of the public. The submission noted that numerous endocrine-related medical conditions are often overlooked, which can lead to unnecessary suffering for patients as these are often treatable conditions. The group also highlighted the importance of diagnosing and treating children early to reduce needless medical hardships they may face throughout their lifetime. 


\section{Disease Experience}

The ICOSEP submission indicated that, although children who would be treated with mecasermin may appear short compared to others, their health issues run deeper than just height and can affect their entire lives. For instance, simple, everyday activities such as getting out of bed, playing with others, and concentrating on tasks require a large amount of effort. The energy needed for these children to grow and develop properly may not be available for them.

The group emphasized that children with SPIGFD do not choose to be lazy, incapable of learning, or exhausted, but "this is their normal." Because most children with SPIGFD have lived with it since birth, "they do not have an understanding that their 'normal' is a healthy person's ill." Children may also struggle to comply with requests, and signs of their issues may appear more pronounced as they get older, leading to increased stress. While short stature is a visual indicator, patients who continue untreated may also have additional comorbidities relating to "heart strength, lung capacity, and others," which can cause lifelong damage.

\section{Experiences With Currently Available Treatments}

The ICOSEP submission noted that there are currently no substitutes for mecasermin for the treatment of SPIGFD and that children who remain untreated do not improve on their own. It also stressed the importance of being able to access medical treatments like mecasermin, which can have a lifelong impact on the health of these patients.

\section{Improved Outcomes}

The patient group submission described the benefit to be "astronomical" for children with SPIGFD who receive medical treatment. It reiterated that this condition is more than just a cosmetic issue related to growth but can also affect the body's internal systems, heart, lungs, and bones. The group stated, "They simply want to have energy to be a child, laugh, play and grow up to be a healthy adult."

In addition, ICOSEP encourages health care decision-makers to review and consider the importance of this treatment, pointing out that mecasermin is available in the US and some European countries. The submission added that "the short-term costs would not compare to the lifetime of specialized care required by those untreated."

\section{Clinician Input \\ Input From the Clinical Expert Consulted by CADTH}

All CADTH review teams include at least 1 clinical specialist with expertise in the diagnosis and management of the condition for which the drug is indicated. Clinical experts are a critical part of the review team and are involved in all phases of the review process (e.g., providing guidance on the development of the review protocol; assisting in the critical appraisal of clinical evidence; interpreting the clinical relevance of the results; and providing guidance on the potential place in therapy). The following input was provided by a clinical specialist with expertise in the diagnosis and management of SPIGFD.

\section{Unmet Needs}

For patients with Laron syndrome (i.e., true genetic IGF-1 deficiency secondary to GH receptor mutation or deletion), with mutations of the IGF-1 gene, with GH antibodies, and for those 
clinically diagnosed with IGF-1 deficiency and undetectable insulin-like growth factor binding protein without known causes, there is no other treatment option aside from mecasermin.

\section{Place in Therapy}

There is no existing treatment for SPIGFD in Canada. Mecasermin would be a first-line treatment in cases where there is either a clear genetic or biochemical diagnosis of SPIGFD or GH antibodies. Mecasermin would cause a shift in the current treatment paradigm at the diagnostic level, because in many jurisdictions there is limited access to $\mathrm{GH}$ antibody testing, GH-IGF-1 axis genetic panels, or a reliable test to assess IGF-1 response to $\mathrm{GH}$. At present, idiopathic short stature (i.e., with no identifiable cause) is treated with $\mathrm{GH}$. As patients with SPIGFD are not expected to respond to high doses of $\mathrm{GH}$, the treatment would be discontinued due to poor response, without any alternatives.

It will be important to ensure appropriate diagnostic criteria are used for SPIGFD and that diagnostic tools are available to avoid misdiagnosis and overtreatment. This is particularly true because patients with GH deficiency will also likely respond to mecasermin. In patients with milder forms of IGF-1 deficiency, which is difficult to diagnose clinically or biochemically (compared to those with true Laron syndrome or clear biochemical evidence of $\mathrm{GH}$ resistance or $\mathrm{GH}$ antibodies), it is suggested that $\mathrm{GH}$ should be tried first.

\section{Patient Population}

Patients likely to demonstrate a clinically meaningful response to mecasermin treatment include those with SPIGFD due to a genetic defect of the GH-IGF-1 pathway, and those who have $\mathrm{GH}$ antibodies. These patients will have specific facial signs of $\mathrm{GH}$ receptor abnormalities and a slow height velocity. Tools to identify patients with SPIGFD who would benefit from mecasermin treatment should include genetic testing, measurements of $\mathrm{GH}$ antibodies and $\mathrm{GH}$-binding globulin, or clear biochemical diagnostic tests, such as measurements of IGF-1 response to GH. Other causes of short stature (e.g., nutritional causes and chronic diseases) need to be ruled out.

\section{Assessing Response to Treatment}

The most important outcomes for assessing treatment response include height velocity and final adult height. Although rarely investigated, HRQoL is also important. In untreated patients with Laron syndrome, the final adult height will be $120 \mathrm{~cm}$ to $130 \mathrm{~cm}$. Final height is improved with mecasermin treatment but remains well below normal. The treatment response is not as good as that with $\mathrm{GH}$ in patients with GH deficiency.

\section{Discontinuing Treatment}

Treatment should be discontinued when the height velocity is less than $1 \mathrm{~cm}$ over 6 months or less than $2 \mathrm{~cm}$ over 1 year with a bone age that is considered in the near-adult range (i.e., older than 14 years in females and older than 16 years in males). Otherwise, the criteria for discontinuation of treatment for patients with an unsatisfactory treatment response need to be clarified.

\section{Prescribing Conditions}

Patients with SPIGFD who are treated with mecasermin should be followed by a pediatric endocrinologist. At present, patients are likely underdiagnosed; however, the focus for treatment with mecasermin should be on misdiagnosis and overtreatment. Patients should meet the minimum criteria as defined in the product monograph: a height SDS of no greater than -3.0; basal IGF-1 levels below the 2.5th percentile for age and sex; GH sufficiency; and 
the exclusion of secondary forms of IGF-1 deficiency. It is possible that these standard criteria may result in misdiagnosis and overtreatment. As such, additional testing (i.e., genetic testing, $\mathrm{GH}$ antibody testing, low GH-binding globulin measurements, or clear biochemical evidence of poor IGF-1 response to GH) should be considered.

\section{Additional Considerations}

Given the challenges related to a definitive diagnosis of SPIGFD in the absence of a recognized genetic cause, it is suggested that treatment decisions be made by a panel of clinical experts (i.e., in an academic centre or part of a committee where cases are collegially discussed) on a case-by-case basis to avoid misdiagnosis and overtreatment. This may not be feasible, as few Canadian clinicians have direct experience with the diagnosis and treatment of SPIGFD; however, physicians with expertise in managing pediatric endocrine growth disorders may also be qualified to contribute. Treatment decisions would be based on relevant anthropometric and laboratory findings (as available) and clinical expert opinion.

\section{Drug Program Input}

The drug programs provide input on each drug being reviewed through CADTH's reimbursement review processes by identifying issues that may affect their ability to implement a recommendation. The implementation questions and corresponding responses from the clinical expert consulted by CADTH are summarized in Table 4.

\section{Clinical Evidence}

The clinical evidence included in the review of mecasermin is presented in 2 sections. The first section, the systematic review, includes pivotal studies provided in the sponsor's submission to CADTH and Health Canada, as well as those studies that were selected according to an a priori protocol. The second section includes additional relevant studies that were considered to address important gaps in the evidence included in the systematic review. Because mecasermin is the only available treatment for SPIGFD, there was no search for indirect evidence in this review.

\section{Systematic Review (Pivotal and Protocol-Selected Studies) Objectives}

To perform a systematic review of the beneficial and harmful effects of mecasermin $10 \mathrm{mg} /$ $\mathrm{mL} \mathrm{SC}$ injection for the treatment of growth failure in children and adolescents (aged 2 to 18 years) with confirmed SPIGFD.

\section{Methods}

Studies selected for inclusion in the systematic review included pivotal studies provided in the sponsor's submission to CADTH and Health Canada, as well as those meeting the selection criteria presented in Table 5. Outcomes included in the CADTH review protocol reflect outcomes considered to be important to patients, clinicians, and drug plans. 
Table 4: Summary of Drug Plan Input and Clinical Expert Response

Drug program implementation questions
There is a group of $\mathrm{GH}$ insensitivity syndromes that could
respond to mecasermin, but some may also respond to
relatively high doses of $\mathrm{GH}$.
- Is molecular testing for $\mathrm{GH}$ receptor gene mutations
available across the country to definitely diagnose
SPIGFD?
- Should eligibility criteria for mecasermin include the
requirement to trial 3 to 6 months of GH unless SPIGFD is
definitely diagnosed (e.g., by molecular testing?)

In Study 1419 , a total of 21 patients (out of 92 ) received leuprolide to delay puberty and prolong the growth period in an attempt to achieve a greater adult height.

- Will leuprolide be used in clinical practice to delay puberty and prolong the growth period in patients who were treated with mecasermin?

The primary efficacy end points in Study 1419 was height velocity and near-adult height compared to baseline.

- Once a patient is started on mecasermin, is it appropriate to continue treatment with mecasermin if they do not achieve height velocity outcomes similar to the clinical trial at a yearly exam (e.g., $8 \pm 2.3 \mathrm{~cm}$ per year during year $1)$ ? Or are growth targets considered patient-specific so that, once therapy is started, it is essentially continued until the patient is 18 years old?

In the data submitted, the mean follow-up period of patients was 8 years.

- If patients are responding to mecasermin, at what point should it be discontinued? After 8 years based on the mean follow-up period from the clinical trials? At 18 years old because mecasermin is not indicated for patients beyond that age? After X-ray-confirmed closure of the epiphyseal plates?

\section{Clinical expert response}

Patients with GH deficiency will likely respond to mecasermin. Conversely, patients with mild primary insulin-like growth factor deficiency may also respond to high doses of $\mathrm{GH}$. The Pediatric Endocrine Society guidelines recommend starting mecasermin directly for patients with hormone-signalling defects known to be unresponsive to $\mathrm{GH}$ treatment; this includes patients with very low or undetectable levels of GHBP and/or proven $\mathrm{GH}$ receptor mutations, GH-neutralizing antibodies, and other known gene mutations associated with SPIGFD (e.g., STAT5 $b$ gene mutations and IGF-1 gene deletion or mutation)..$^{14}$ The clinical expert consulted by CADTH stated that molecular testing for $\mathrm{GH}$ receptor gene mutations (or other known mutations associated with SPIGFD) would be ideal; however, molecular testing is available but difficult to access in some Canadian jurisdictions and the cost is high. Moreover, molecular testing will always be limited to known genetic causes of SPIGFD. ${ }^{14}$ The guidelines note that genetic testing is desirable for patients for whom diagnostic uncertainty is problematic, to better inform the treatment plan. ${ }^{14}$ For patients with unexplained IGF-1 deficiency, the guidelines state that a trial of $\mathrm{GH}$ is reasonable; however, the guidelines do not provide recommendations for the length of the trial.

Although not an approved indication, some clinicians may prescribe leuprolide to patients with SPIGFD to increase the length of the growth period (specifically in patients near the end of the growth period or for whom bone age is rapidly increasing). The decision to prescribe leuprolide would occur on a case-by-case basis.

Once a patient has begun treatment with mecasermin, treatment should continue so long as the gain in height is $\geq 1 \mathrm{~cm}$ per 6 months or $\geq 2 \mathrm{~cm}$ per year or they have reached near-adult height based on bone age criteria (i.e., bone age of $>16$ years for males and $>14$ years for females). Typically, the best response will be observed in the first year of treatment. Bone age is more informative than chronological age when deciding when to stop treatment.

Treatment should be discontinued when the gain in height is $<1$ $\mathrm{cm}$ per 6 months or $<2 \mathrm{~cm}$ per year, or patients have reached near-adult height based on bone age criteria (i.e., bone age of $>16$ years for males and $>14$ years for females). 
Drug program implementation questions

Laron syndrome has clinical manifestations outside of lower height - small head circumference, characteristics faces with saddle nose and prominent forehead, delayed skeletal maturation, small genitalia and testes, short limb length compared with trunk length, and abnormal body composition, with osteopenia and obesity.

- Is there evidence that mecasermin provides benefit for these other manifestations?

The product monograph clearly warns that there have been post-marketing reports of both benign and malignant neoplasms in children and adolescents who have received treatment with mecasermin because IGF-1 plays a role in the initiation and progression of tumours.

- Do the benefits of mecasermin (i.e., increased height) outweigh the potential harms of use?
Clinical expert response

The primary aim of mecasermin treatment is to improve height velocity and final adult height in patients with SPIGFD. There is no evidence from trials for a beneficial effect of mecasermin on other clinical manifestations of Laron syndrome. An observational study of 5 patients with Laron syndrome found that 3 to 10 months of treatment with recombinant IGF-1 resulted in a marked increase in head circumference, increased body weight, and a reduction in subcutaneous body fat. ${ }^{11}$

There are inadequate data to draw strong conclusions about the ratio of benefits to harms for patients treated with mecasermin for SPIGFD. In clinical practice, the ratio of benefits to harms needs to be discussed individually with each patient and their parent or caregiver. To make an informed decision, patients and their parents or caregivers should be explained that the risk of benign and malignant tumours in children with SPIGFD is lower than for healthy children without SPIGFD (because IGF-1 plays a role in the initiation and progression of benign and malignant tumours). ${ }^{12}$ Treatment with mecasermin may increase the risk of benign and malignant tumours, although the relationship between mecasermin and the risk of benign and malignant tumours is uncertain. Superior data are available in patients with $\mathrm{GH}$ deficiency and the long-term outcomes in terms of malignancy are reassuring.

A Canadian registry may be useful to monitor benefits and long-term harms in patients treated with mecasermin. The sponsor reported that as of March 10, 2019, a total of 36 cases of benign or malignant neoplasms were identified in their global safety database. In $67 \%$ of cases (24 of 36 ), mecasermin was administered in an off-label indication. In $25 \%$ of cases (9 of 36), mecasermin was used in a higher-than-recommended dose. For the 19 malignant neoplasia cases, 16 (84\%) reported an off-label use and $6(32 \%)$ reported an off-label dose. Because Health Canada requires that mecasermin be distributed through the sponsor's patient support program, the risk of off-label use and/or of dosing higher than recommended is fully mitigated.

$\mathrm{GH}=$ growth hormone; GHBP = growth hormone binding protein; IGF-1 = insulin-like growth factor 1; SPIGFD = severe primary insulin-like growth factor 1 deficiency .

The literature search for clinical studies was performed by an information specialist using a peer-reviewed search strategy according to the Peer Review of Electronic Search Strategies checklist. ${ }^{33}$

Published literature was identified by searching the following bibliographic databases: MEDLINE All (1946囚) via Ovid and Embase (1974『) via Ovid. All Ovid searches were run simultaneously as a multi-file search. Duplicates were removed using Ovid deduplication for multi-file searches, followed by manual deduplication in Endnote. The search strategy comprised both controlled vocabulary, such as the National Library of Medicine's MeSH (Medical Subject Headings), and keywords. The main search concepts were Increlex (mecasermin). Clinical trials registries searched included the US National Institutes of Health's clinicaltrials.gov, Health Canada's Clinical Trials Database, and the European Union Clinical Trials Register. 
Search filters developed by CADTH were applied to limit retrieval to randomized controlled trials or controlled clinical trials. Where possible, retrieval was limited to the human population. Retrieval was not limited by publication date or by language. Conference abstracts were excluded from the search results. See Appendix 1 for the detailed search strategies.

\section{Table 5: Inclusion Criteria for the Systematic Review}

\begin{tabular}{|c|c|}
\hline Criteria & Description \\
\hline Patient population & $\begin{array}{l}\text { Children and adolescents aged } 2 \text { to } 18 \text { years with growth failure due to confirmed SPIGFD } \\
\text { SPIGFD is defined as a height standard deviation score } \leq-3.0 \text {, basal IGF-1 levels below the } 2.5 \text { th } \\
\text { percentile for age and sex, GH sufficiency, and exclusion of secondary forms of IGF-1 deficiency (e.g., } \\
\text { malnutrition, hypopituitarism, hypothyroidism, chronic treatment with pharmacologic doses of anti- } \\
\text { inflammatory steroids) } \\
\text { SPIGFD includes patients with mutations of the GHR gene/Laron's syndrome, post-GHR signalling } \\
\text { pathway, and IGF-1 gene defects } \\
\text { Subgroups: } \\
\text { - Age, including chronological age (years) and/or pre-treatment bone age (years) } \\
\text { - Pubertal stage (e.g., } 1 \text { vs. } 2 \text { vs. } 3 \text { ) } \\
\text { - Pre-treatment height (e.g., height velocity, height SDS) } \\
\text { - Prior treatment with mecasermin (yes vs. no) } \\
\text { - Prior treatment with GH treatment (yes vs. no; by dosage of GH treatment) } \\
\text { - Concomitant therapies (yes vs. no) } \\
\text { - Etiology of SPIGFD (GH receptor defects/Laron's syndrome, post-GHR signalling pathway, or IGF-1 gene } \\
\text { defects; with vs. without GH antibodies) }\end{array}$ \\
\hline Intervention & $\begin{array}{l}\text { Mecasermin subcutaneous injection (starting dose of } 0.04 \mathrm{mg} / \mathrm{kg} \text { to } 0.08 \mathrm{mg} / \mathrm{kg}[40 \mathrm{mcg} / \mathrm{kg} \text { to } 80 \mathrm{mcg} / \\
\mathrm{kg}] \text { twice daily, increasing to maximum } 0.12 \mathrm{mg} / \mathrm{kg}[120 \mathrm{mcg} / \mathrm{kg}] \text { twice daily if tolerated) }\end{array}$ \\
\hline Comparators & Best supportive care with or without placebo \\
\hline Outcomes & $\begin{array}{l}\text { Efficacy outcomes: } \\
\text { - Height (e.g., height velocity, final height, height SDS, predicted adult height) } \\
\text { - HRQoL (e.g., QoLiSSY, PedsQL) and HRQoL by gain in height } \\
\text { - Skeletal maturation (e.g., bone age, bone age SDS) } \\
\text { - Body mass or composition (e.g., weight, body weight SD score, body mass index, body fat mass, lean } \\
\text { body mass) } \\
\text { Harms outcomes: } \\
\text { - Adverse events } \\
\text { - Serious adverse events } \\
\text { - Withdrawals due to adverse events } \\
\text { - Mortality } \\
\text { - Notable harms (e.g., hypoglycemia, lymphoid tissue hypertrophy, injection site lipohypertrophy, benign } \\
\text { or malignant neoplasia, intracranial hypertension, nephrolithiasis, orthopedic pain) }\end{array}$ \\
\hline Study design & Published and unpublished phase III and IV randomized controlled trials \\
\hline
\end{tabular}

$\mathrm{GH}=$ growth hormone; GHR = growth hormone receptor; HRQoL = health-related quality of life; IGF-1 = insulin-like growth factor 1 ; PedsQL = Pediatric Quality of Life

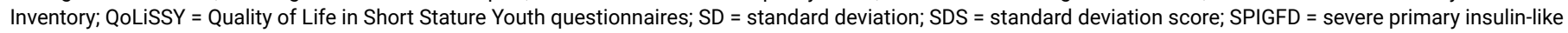
growth factor 1 deficiency; vs. = versus. 
The initial search was completed on July 13, 2021. Regular alerts updated the search until the meeting of the CADTH Canadian Drug Expert Committee on November 24, 2021.

Grey literature (literature that is not commercially published) was identified by searching relevant websites from the Grey Matters: A Practical Tool For Searching Health-Related Grey Literature checklist. ${ }^{34}$ Included in this search were the websites of regulatory agencies (US FDA and European Medicines Agency). Google was used to search for additional internetbased materials. Appendix 1 provides more information on the grey literature search strategy.

These searches were supplemented by reviewing bibliographies of key papers and through contacts with appropriate experts. In addition, the sponsor of the drug was contacted for information regarding unpublished studies.

Two CADTH clinical reviewers independently selected studies for inclusion in the review based on titles and abstracts, according to the predetermined protocol. Full-text articles of all citations considered potentially relevant by at least 1 reviewer were acquired. Reviewers independently made the final selection of studies to be included in the review, and differences were resolved through discussion.

\section{Findings From the Literature}

One study ${ }^{15-17}$ was identified from the literature for inclusion in the systematic review (Figure 1). The included study is summarized in Table 6. A list of excluded studies is presented in Appendix 2.

\section{Description of Study}

One pivotal trial (FS0903S; hereafter referred to as Study 1419) met the inclusion criteria for this review. ${ }^{15-17}$ Study 1419 was a phase III open-label, multi-centre, single-arm, investigatorsponsored trial with linked data from 4 predecessor studies (F0206S, F0375G, F0632G, and F0671G). Of the predecessor studies, 3 were open-label single-arm trials (F0206S, F0632G, and F0671G), 1 was investigator-sponsored (F0206S), and 1 was multi-centre (F0671G). Study F0375G $(n=8)$ was a 27-month double-blind, placebo-controlled crossover trial that included 6 months of mecasermin or placebo treatment followed by a 3-month washout period, a 6-month crossover period, and a 12-month open-label extension. Simple randomization was used in Study F0375G to assign patients to the initial treatment group. Because height velocity was an objective end point and the long-term height velocities in the other 4 studies were expected to be substantially greater than both baseline and historical results in untreated children with SPIGFD, a randomized controlled group was deemed unnecessary in subsequent studies.

The purpose of this series of studies was to determine the safety and efficacy of long-term IGF-1 replacement therapy with mecasermin SC for the treatment of growth failure in children with SPIGFD. The linking of data from patients who participated in earlier trials allowed for each patient's data to be analyzed both individually and in aggregate with the rest of the treatment population. Many of the patients enrolled in Study 1419 had been continuously treated with mecasermin for many years and had transferred from 1 protocol to another when 1 study ended. All patients enrolled in studies F0206S, F0375G, and F0632G were later enrolled in study F0671 G. All but 1 patient enrolled in F0671G were later enrolled in Study 1419 (Figure 2). The integrated study report includes results for patients enrolled in the 5 company- and investigator-sponsored studies $(n=92)$ that used Genentech and subsequently Tercica (now Ipsen Biopharmaceuticals, Inc.) drug products at 2 investigative sites in the 
US in conjunction with sites in 23 other countries worldwide. Two patients from Canada were enrolled.

\section{Populations}

\section{Inclusion and Exclusion Criteria}

Patients with growth failure due to SPIGFD associated with either GHR defects or GH-deletion defects and anti-GH antibodies were eligible for enrolment. The eligibility criteria for Study 1419 were similar to those for the other 4 studies. Eligible patients were those who had a height SDS of less than -2 for age and sex; had a growth rate of less than the 50 th percentile for age and sex for more than 6 months before the study start; had an IGF-1 SDS of less than -2 for age and sex; were more than 18 months old; and had open epiphyses. For those with GHIS and Laron syndrome, eligible patients needed a random or stimulated GH level of greater than $10 \mathrm{ng} / \mathrm{mL}$ and failure to increase IGF- 1 by $50 \mathrm{ng} / \mathrm{mL}$ in response to exogenous GH during an IGF-1 generation test. For those with GHIS and GH gene deletion, eligibility required the presence of $\mathrm{GH}$ antibodies to exogenous $\mathrm{GH}$ with a binding capacity of greater than $10 \mathrm{mcg} / \mathrm{mL}$. Ineligible patients were those with active malignancy or any history of

\section{Figure 1: Flow Diagram for Inclusion and Exclusion of Studies}

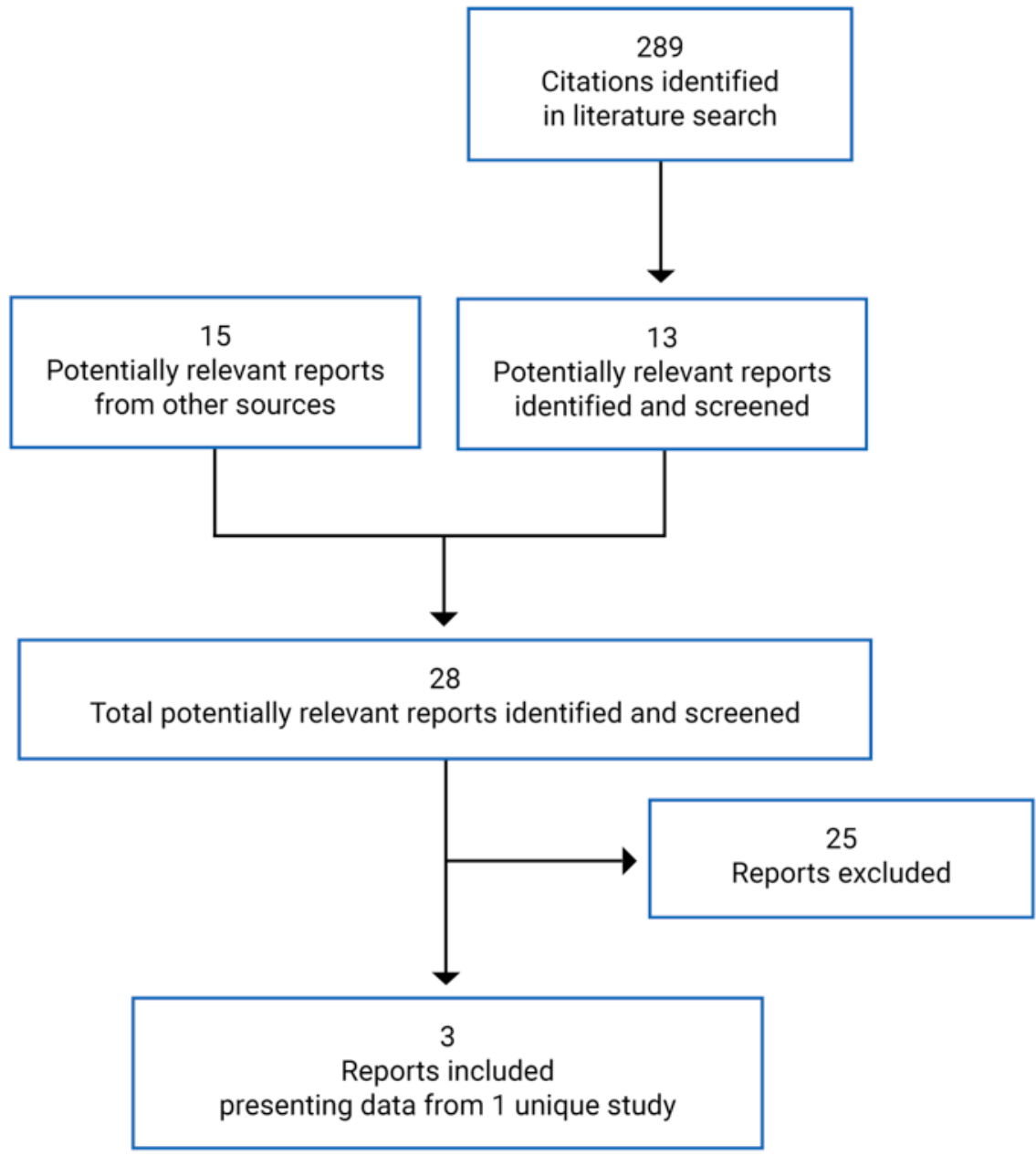


Table 6: Details of Study 1419

\section{Study 1419}

Designs and populations

\begin{tabular}{|c|c|}
\hline Study design & $\begin{array}{l}\text { Phase III multi-centre, open-label, single-arm trial with linked data from } 4 \text { predecessor studies (F0206S, } \\
\text { F0375G, F0632G, and F0671G)a }\end{array}$ \\
\hline Locations & 24 countries \\
\hline Study dates & May 20, 1991, to December 15, 2011 \\
\hline Enrolled (N) & 92 \\
\hline Analyzed (N) & 81 (efficacy outcomes); 92 (safety outcomes) \\
\hline Inclusion criteria & $\begin{array}{l}\text { - Individuals with growth failure due to SPIGFD associated with either } \mathrm{GH} \text { receptor defects or GH } \\
\text { gene-deletion defects and anti-GH antibodies } \\
\text { - Height SDS <-2 for age and sex } \\
\text { - Growth rate of < 50th percentile for age and sex for }>6 \text { months before study start } \\
\text { - IGF-1 SDS of <-2 for age and sex } \\
\text { - Age > } 18 \text { months } \\
\text { - Open epiphyses } \\
\text { - For GHIS, Laron syndrome only: random or stimulated GH level of }>10 \mathrm{ng} / \mathrm{mL} \text { and failure to increase } \\
\text { IGF-1 by } 50 \mathrm{ng} / \mathrm{mL} \text { in response to exogenous } \mathrm{GH} \text { during an IGF- } 1 \text { generation test } \\
\text { - For GHIS, GH gene-deletion type only: presence of GH antibodies to exogenous } \mathrm{GH} \text { with a binding } \\
\text { capacity of }>10 \mathrm{mcg} / \mathrm{mL}\end{array}$ \\
\hline Exclusion criteria & $\begin{array}{l}\text { - Active malignancy or any history of malignancy } \\
\text { - Growth failure due to other reasons: disorders of genitourinary, cardiopulmonary, gastrointestinal, or } \\
\text { nervous system, other endocrine disorders, nutritional or vitamin deficiencies, or chondrodystrophies } \\
\text { - Treatment with any corticosteroids or other medications that influence growth } \\
\text { - Clinically significant electrocardiogram abnormality or a history of clinically significant cardiac } \\
\text { arrhythmia }\end{array}$ \\
\hline \multicolumn{2}{|r|}{ Drugs } \\
\hline Intervention & $\begin{array}{l}\text { - } 60 \mathrm{mcg} / \mathrm{kg} \text { to } 120 \mathrm{mcg} / \mathrm{kg} \text { mecasermin SC twice daily (morning and evening) within } 30 \text { minutes of a } \\
\text { meal (with doses up to } 160 \mathrm{mcg} / \mathrm{kg} \mathrm{SC} \text { twice daily in some pubertal patients) } \\
\text { - Naive-to-treatment patients generally started at } 60 \mathrm{mcg} / \mathrm{kg} \text { to } 80 \mathrm{mcg} / \mathrm{kg} \mathrm{SC} \text { twice daily for } 1 \text { or } 2 \\
\text { weeks and then increased to } 120 \mathrm{mcg} / \mathrm{kg} \mathrm{SC} \mathrm{twice} \mathrm{daily} \mathrm{as} \mathrm{tolerated}\end{array}$ \\
\hline Comparator(s) & $\begin{array}{l}\text { - Within-patient pre-treatment control } \\
\text { - Historical controls with untreated SPIGFD (for attainment of near-adult height only) } \\
\text { - Placebo (Study F0375G only) }\end{array}$ \\
\hline \multicolumn{2}{|r|}{ Duration } \\
\hline Longest follow-up & 19 years \\
\hline \multicolumn{2}{|r|}{ Outcomes } \\
\hline Primary end points & $\begin{array}{l}\text { - Height velocity } \\
\text { - Near-adult height }{ }^{\mathrm{d}} \\
\text { - Estimated improvement in adult height }\end{array}$ \\
\hline
\end{tabular}


Study 1419

\begin{tabular}{|c|c|}
\hline $\begin{array}{l}\text { Secondary and } \\
\text { exploratory end points }\end{array}$ & $\begin{array}{l}\text { - Height velocity SDS } \\
\text { - Height SDS } \\
\text { - Change in bone age relative to change in chronological age } \\
\text { - BMI SDS } \\
\text { - AE, SAE, WDAE, mortality, notable harms }\end{array}$ \\
\hline & Notes \\
\hline Publications & $\begin{array}{l}\text { Chernausek et al. }(2007)^{17} \\
\text { Backeljauw et al. }(2013)^{16}\end{array}$ \\
\hline
\end{tabular}

$\mathrm{AE}=$ adverse event; $\mathrm{BMI}=$ body mass index; $\mathrm{GH}=$ growth hormone; $\mathrm{GHIS}=$ growth hormone insensitivity syndrome; IGF-1 = insulin-like growth factor $1 ; \mathrm{SAE}=$ serious adverse event; SC = subcutaneous; $S D$ = standard deviation; $S D S$ = standard deviation score; SPIGFD = severe primary insulin-like growth factor 1 deficiency; WDAE = withdrawal due to adverse event.

${ }^{\mathrm{a}}$ Three of the 4 predecessor trials (studies F0206S, F0632G, and F0671G) were open-label and 1 was a double-blind, placebo-controlled crossover trial (Study F0375G). All patients enrolled in studies F0206S, F0375G, and F0632G were later enrolled in Study F0671G. With 1 exception, all patients enrolled in F0671G were later enrolled in Study 1419.

bIn studies F0206S, F0375G, and F0632G, mecasermin was provided in single-use vials containing $1.2 \mathrm{~mL}$ of sterile solution that delivered $5 \mathrm{mg}$ mecasermin per $1 \mathrm{~mL}$ in citrate/ $\mathrm{NaCl}$ (pH 6.0). In Study F0671G and initially in Study 1419, mecasermin was provided in $10 \mathrm{~mL}$ glass vials containing a sterile benzyl alcohol-preserved, acetate/ $\mathrm{NaCl}$-buffered solution of $70 \mathrm{mg}$ mecasermin per $7 \mathrm{~mL}(10 \mathrm{mg} / \mathrm{mL})$. Subsequently the mecasermin was provided in $5 \mathrm{~mL}$ glass vials containing a sterile benzyl alcoholpreserved solution of $40 \mathrm{mg}$ of mecasermin per $4 \mathrm{~mL}$. Twenty-one patients received concomitant therapy with leuprolide to delay puberty and prolong the growth period. cIn Study F0206S, mecasermin was started at a low dose and increased to $120 \mathrm{mcg} / \mathrm{kg} \mathrm{SC}$ twice daily. In Study F0375G, mecasermin was administered at 80 mcg/kg SC twice daily for 1 day in hospital, and if well tolerated, at $120 \mathrm{mcg} / \mathrm{kg} \mathrm{SC}$ twice daily for 2 additional days before discharge. In Study F0632G, mecasermin was administered at $80 \mathrm{mcg} / \mathrm{kg} \mathrm{SC}$ twice daily for 3 days in hospital with careful glucose monitoring for 1 year following discharge. In Study F0671G, mecasermin was administered at 80 $\mathrm{mcg} / \mathrm{kg}$ to $120 \mathrm{mcg} / \mathrm{kg} \mathrm{SC}$ twice and the patients continued this regimen in study 1419 .

${ }^{\mathrm{d}}$ Patients were considered to have attained near-adult height when the most recent bone age was at least 14 years for females and 16 years for males.

${ }^{e}$ Mean \pm SD of the difference between the observed increase in height vs. that expected from Laron et al. (1993). ${ }^{18}$

Source: Clinical Study Report for Study $1419 .{ }^{15}$

malignancy, growth failure due to other reasons, treatment with any corticosteroids or other medications that influence growth, and a clinically significant electrocardiogram abnormality or a history of a clinically significant cardiac arrhythmia.

\section{Baseline Characteristics}

Baseline characteristics were reported for the safety analysis (Table 7). This includes all 92 patients enrolled, including those who were naive to mecasermin upon entering the predecessor studies or Study $1419(n=83$, with baseline data reflecting those recorded for the predecessor study for patients who participated in a predecessor study before entering

\section{Figure 2: Sequence of Patient Enrolment Into Study 1419}

\begin{tabular}{|c|c|c|c|c|c|c|c|c|}
\hline New & 8 & 8 & 5 & & 2 & \multicolumn{3}{|c|}{69} \\
\hline & $t$ & & & $n=8$ & & \multirow{4}{*}{60 naĩve - } & \multirow{4}{*}{\multicolumn{2}{|c|}{$\begin{array}{c}\leftarrow 9 \text { subjects previously } \\
\text { received Pharmacia } \\
\text { mecasermin }\end{array}$}} \\
\hline & $\begin{array}{l}206 s \\
n=8\end{array}$ & & & & & & & \\
\hline & & \multirow{2}{*}{$\begin{array}{l}375 g \\
n=8\end{array}$} & & $n=7$ & & & & \\
\hline & & & & & & & & \\
\hline & & $n=$ & \multirow{2}{*}{$\begin{array}{l}6329 \\
n=\end{array}$} & $\mathrm{n}=6$ & \multirow{2}{*}{$\begin{array}{l}671 \mathrm{~g} \\
\mathrm{n}=2\end{array}$} & $n=2$ & \multirow{2}{*}{$\begin{array}{l}1419 \\
n=9\end{array}$} & \\
\hline & & & & & & & & \\
\hline Cumulative & 8 & 16 & 21 & & 23 & & 92 & \\
\hline
\end{tabular}

Source: Clinical Study Report for Study $1419 .{ }^{15}$ 
Study 1419) and those who had previously been treated with Pharmacia mecasermin ( $n=$ 9). The mean chronological age of patients at baseline was 7.6 years $(S D=4.3)$, with a range of 1.7 to 17.5 years. By contrast, mean bone age was 3.8 years $(S D=2.8)$. More than half of patients were male $(n=53 ; 58 \%)$ and the etiology of GHIS for most patients was Laron syndrome $(n=82 ; 89 \%)$. Most patients began treatment at pubertal stage $1(n=79 ; 86 \%)$. Few $(n=9 ; 10 \%)$ had received prior IGF-1 therapy. Most patients (84\%) were White.

All patients had severe short stature, with a mean height and height SDS of $88.5 \mathrm{~cm}$ (SD = $20.7)$ and $-6.7(S D=1.9)$, respectively. Mean pre-treatment height velocity and height velocity SDS were $2.6 \mathrm{~cm}$ per year $(S D=1.8)$ and $-3.2(S D=1.8)$, respectively. Patients started treatment at a mean body weight of $14.1 \mathrm{~kg}(\mathrm{SD}=8.8)$. The mean $\mathrm{BMI}$ and BMI SDS at baseline were $16.6 \mathrm{~kg} / \mathrm{m}^{2}(\mathrm{SD}=2.8)$ and $-0.2(\mathrm{SD}=1.2)$, respectively.

Serum IGF-1 levels $(\mathrm{ng} / \mathrm{mL}$ ) and IGF-1 SDSs were below normal for all patients (mean of 25.3 $\mathrm{ng} / \mathrm{mL}[\mathrm{SD}=25.8]$ and -4.2 [SD = 1.8], respectively). Among 46 patients who received an IGF1 generation test at baseline, the mean maximum serum IGF-1 and serum IGF-1 SDS from the test were $24.0 \mathrm{ng} / \mathrm{mL}(\mathrm{SD}=28.1)$ and $-4.4(\mathrm{SD}=2.2)$, respectively. The mean maximum $\mathrm{GH}$ level was $53.8 \mathrm{ng} / \mathrm{mL}(\mathrm{SD}=43.2)$ and the mean enrolment maximum $\mathrm{GH}$ level for the subgroup of patients $(n=76)$ with Laron phenotype was $56.0 \mathrm{ng} / \mathrm{mL}(S D=43.0)$.

\section{Interventions}

Patients received mecasermin $60 \mathrm{mcg} / \mathrm{kg}$ to $120 \mathrm{mcg} / \mathrm{kg} \mathrm{SC}$ twice daily. Active drug and placebo (in Study F0375G) were initially provided by Genentech Inc., later by Tercica Inc., and then by Ipsen Biopharmaceuticals, Inc. In studies F0206S, F0375G, and F0632G, mecasermin was provided in single-use vials containing $2.2 \mathrm{~mL}$ of sterile solution that delivered $5 \mathrm{mg}$ mecasermin per $1 \mathrm{~mL}$ in citrate/ $\mathrm{NaCl}$ (pH 6.0). Placebo in Study F0375G was provided as a single-use vial containing $1.2 \mathrm{~mL}$ of sterile solution of mecasermin excipient without an active ingredient. In studies F0671G and initially Study 1419, mecasermin was provided in 10 $\mathrm{mL}$ glass vials containing a sterile benzyl alcohol-preserved, acetate/ $\mathrm{NaCl}$-buffered solution of $70 \mathrm{mg}$ mecasermin per $7 \mathrm{~mL}(10 \mathrm{mg} / \mathrm{mL}$, pH 5.4). Subsequently, the mecasermin was provided in $5 \mathrm{~mL}$ glass vials containing a sterile benzyl alcohol-preserved solution of $40 \mathrm{mg}$ mecasermin per $4 \mathrm{~mL}$ (pH 5.2 to 5.6).

Patients naive to mecasermin treatment generally started mecasermin at $60 \mathrm{mcg} / \mathrm{kg}$ to 80 $\mathrm{mcg} / \mathrm{kg}$ SC twice daily for 1 to 2 weeks and then increased to $120 \mathrm{mcg} / \mathrm{kg} \mathrm{SC}$ twice daily as tolerated. Patients and their parents or guardians were instructed to administer injections in the morning and evening, within 30 minutes of a meal. Tolerability was generally closely monitored across the studies at the beginning of treatment via blood glucose monitoring, and in some cases, doses were temporarily reduced. Injections were to be withheld if the patient was unable to eat due to illness.

Exceptions to standard dosing occurred during the studies. Patients in Study F0206S began mecasermin treatment at a dosage of $40 \mathrm{mcg} / \mathrm{kg} \mathrm{SC}$ twice daily and increased over 2 days to $120 \mathrm{mcg} / \mathrm{kg}$ SC twice daily. In response to a request by the US FDA, 6 naive-to-treatment patients in Study F0632G received mecasermin at $60 \mathrm{mcg} / \mathrm{kg}$ SC twice daily for 1 year. Simultaneously, patients enrolled and treated in Study F0206S had their dosage reduced from $120 \mathrm{mcg} / \mathrm{kg}$ SC twice daily to $80 \mathrm{mcg} / \mathrm{kg}$ SC twice daily, and Study F0375G was unblinded and all patients were assigned a dosage of $80 \mathrm{mcg} / \mathrm{kg}$ SC twice daily. Review of data after 1 year showed the $60 \mathrm{mcg} / \mathrm{kg} \mathrm{SC}$ twice daily dosage to be suboptimal, and mecasermin was 
Table 7: Summary of Baseline Characteristics for Study 1419

\begin{tabular}{|c|c|}
\hline Characteristic & Study $1419(\mathrm{~N}=92)$ \\
\hline Pre-treatment age (years), mean (SD) & $7.6(4.3)$ \\
\hline Minimum to maximum & 1.7 to 17.5 \\
\hline \multicolumn{2}{|c|}{ Sex, n (\%) } \\
\hline Male & $53(58)$ \\
\hline Female & $39(42)$ \\
\hline \multicolumn{2}{|c|}{ Race, $n(\%)$} \\
\hline White & $77(84)$ \\
\hline Black & $3(3)$ \\
\hline Hispanic & $6(7)$ \\
\hline Asian & $4(4)$ \\
\hline Other & $2(2)$ \\
\hline \multicolumn{2}{|c|}{ Etiology of GHIS, $n(\%)$} \\
\hline GH gene deletion & $8(9)$ \\
\hline Laron syndrome & $82(89)$ \\
\hline GH antibodies & $1(1)$ \\
\hline Isolated genetic GH deficiency & $1(1)$ \\
\hline \multicolumn{2}{|c|}{ Pubertal stage, $n(\%)$} \\
\hline 1 & $79(86)$ \\
\hline 2 & $4(4)$ \\
\hline 3 & $1(1)$ \\
\hline Unknown & $8(9)$ \\
\hline Previous IGF-1 therapy (yes), n (\%) & $9(10)$ \\
\hline Enrolment IGF-1 (ng/mL), mean (SD) & $25.3(25.8)$ \\
\hline Minimum to maximum & 0.2 to 133.0 \\
\hline Enrolment IGF-1 SDS, mean (SD) & $-4.2(1.8)$ \\
\hline Minimum to maximum & -9.5 to -0.6 \\
\hline Pre-treatment height $(\mathrm{cm})$, mean (SD) & $88.5(20.7)$ \\
\hline Minimum to maximum & 59.7 to 151.3 \\
\hline Pre-treatment height SDS, mean (SD) & to $6.7(1.9)$ \\
\hline Minimum to maximum & -12.1 to -2.8 \\
\hline Pre-treatment height velocity ( $\mathrm{cm} /$ year), mean (SD) & $2.6(1.8)$ \\
\hline Minimum to maximum & 0.0 to 7.9 \\
\hline Pre-treatment height velocity SDS, mean (SD) & $-3.2(1.8)$ \\
\hline
\end{tabular}




\begin{tabular}{|l|c|}
\hline Minimum to maximum & -6.6 to 2.3 \\
\hline Enrolment weight $(\mathrm{kg})$, mean (SD) & 14.1 (8.8) \\
\hline Minimum to maximum & 5.8 to 42.5 \\
\hline Enrolment BMI $\left(\mathrm{kg} / \mathrm{m}^{2}\right)$, mean (SD) & $16.6(2.8)$ \\
\hline Minimum to maximum & 12.8 to 26.4 \\
\hline Enrolment BMI SDS, mean (SD) & $-0.2(1.2)$ \\
\hline Minimum to maximum & -3.1 to 2.2 \\
\hline Bone age (years), mean (SD) & $3.8(2.8)$ \\
\hline Minimum to maximum & 0.1 to 12.3 \\
\hline $\begin{array}{l}\text { Enrolment maximum IGF-1 from IGF-1 generation test }(\mathrm{ng} / \mathrm{mL}), \\
\text { mean (SD) }\end{array}$ & $24.0(28.1)$ \\
\hline Minimum to maximum & 0.5 to 120.0 \\
\hline $\begin{array}{l}\text { Enrolment maximum IGF-1 SD score from IGF-1 generation test, } \\
\text { mean (SD) }\end{array}$ & $-4.4(2.2)$ \\
\hline Minimum to maximum & -9.9 to -0.5 \\
\hline Maximum GH level (ng/mL), mean (SD) & $53.8(43.2)$ \\
\hline Minimum to maximum & 0.5 to 209.0 \\
\hline $\begin{array}{l}\text { Enrolment maximum GH level, Laron type phenotype }(\mathrm{ng} / \mathrm{mL}), \\
\text { mean (SD) }\end{array}$ & $56.0(43.0)$ \\
\hline Minimum to maximum & 1.3 to 209.0 \\
\hline
\end{tabular}

$\mathrm{BMI}=$ body mass index; $\mathrm{GH}$ = growth hormone; GHIS = growth hormone insensitivity syndrome; IGF-1 = insulin-like growth factor 1; SD = standard deviation; SDS = standard deviation score.

Source: Clinical Study Report for Study $1419 .{ }^{15}$

dosed at $80 \mathrm{mcg} / \mathrm{kg}$ SC twice daily to $120 \mathrm{mcg} / \mathrm{kg}$ SC twice daily thereafter. Since 2007, dosages of up to $160 \mathrm{mcg} / \mathrm{kg}$ SC twice daily were used in some pubertal patients.

There were no prohibited concomitant medications, and concomitant medication was taken by 64 patients $(70 \%)$ in the form of anilides $(n=38 ; 30 \%)$, penicillin with extended spectrum $(n=25 ; 27 \%)$, gonadotropin-releasing hormone analogues $(n=21 ; 23 \%)$, cough and cold preparations $(n=14 ; 14 \%)$, antibacterials for systemic use $(n=12 ; 13 \%)$, and systemic hormonal preparations excluding sex hormones $(n=10 ; 10 \%)$. To achieve a greater adult height by delaying puberty and prolonging the growth period, 21 patients (23\%) received leuprolide.

Nine patients received mecasermin from Pharmacia, Inc. in a study for at least 2 weeks before enrolment. They were enrolled in Study 1419 when the Pharmacia study was stopped due to discontinuation of the IGF-1 product. These patients were not included in the efficacy analysis.

\section{Outcomes}

The end points identified in the CADTH review protocol that were assessed in Study 1419 are summarized in this section. Follow-up for outcome measurement was variable in the 
predecessor studies. In F0206S, patients returned monthly for follow-up during the first year of therapy, bimonthly during the second year of therapy, every 3 months during years 3 and 4, and every 6 to 12 months thereafter. Visits alternated between the study centre (University of North Carolina [UNC] at Chapel Hill) and the referring pediatric endocrinologist. In studies F0375G and F0632G, patients returned for follow-up at the study centre (Cincinnati Children's Hospital Medical Centre [CCHMC] in Cincinnati, Ohio) every 3 months. Patients enrolled in studies F0206S, F0375G, or F0632G were offered enrolment in Study F0671G for long-term follow-up. Follow-up visits were reduced to every 6 months at either UNC or CCHMC. In Study 1419 , all patients previously enrolled in Study F0671G returned for follow-up at least annually at either UNC or CCHMC. All patients naive to mecasermin treatment enrolled in Study 1419 were followed by their referring endocrinologist in consultation with investigators at UNC or CCHMC.

Prior to 1998 the studies included in the integrated report were monitored by Genentech. Case report forms (CRFs) were received, tracked, and reviewed for accuracy and completeness by Genentech's in-house data-management group. Following quality checks, including visual CRF review, data were entered into an Informix data-management system using double data entry with third-party arbitration. For Study 1419, Tercica (now Ipsen Biopharmaceuticals, Inc.) developed CRFs to capture the information outlined in the protocol and monitored data at both CCHMC and UNC. All data-management aspects were outsourced to Synteract, Inc., which received, tracked, and reviewed the CRFs for accuracy and completeness. Following quality checks, including visual CRF review, data were entered into the data-management system and subjected to edit checks using SAS software. All data queries following these steps were forwarded to the study sites for resolution. A further quality assurance audit conducted by Synteract found the data to be $99.97 \%$ accurate.

\section{Height \\ Height Velocity and Height Velocity Standard Deviation Score}

Height velocity (i.e., the change in measurement of height from 1 visit to the next) after 1 year in patients naive to mecasermin treatment was a primary efficacy outcome. Height velocity SDS was selected as a secondary efficacy outcome to provide additional statural growth information. Measures of height were performed at UNC and CCHMC using wall-mounted stadiometers. Interval height measurements were also performed by local referring pediatric endocrinologists.

Height velocity SDSs were computed with age- and sex-dependent means and SDs provided

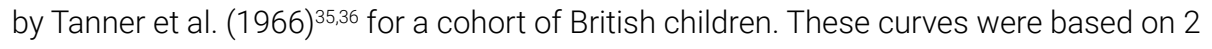
cohorts. For children up to age 11 to 12 years, annual increments of height were available for a random sample of children from the London City Council Study (as few as 19 children, up to a maximum of 220). For adolescents, 3 monthly measurements were taken of 49 boys and 41 girls who were living in a children's home outside of London from the Harpenden Growth Study. ${ }^{35-37}$ Curves depicting the third through the 97th percentile were developed for "average maturers," with third, 50th, and 97th percentiles at peak height velocity highlighted for early and later maturers. ${ }^{37}$

\section{Near-Adult Height and Estimated Improvement in Near-Adult Height}

Near-adult height among patients who achieved near-adult height during the study period was a primary efficacy outcome. Measures of height (as previously described) and bone age were used to determine near-adult height. Height was measured as previously described, and bone age was estimated via radiography of the hand and wrist using the Fels method. ${ }^{38}$ 
The reference group for the Fels method is participants in the Fels Longitudinal Study. ${ }^{39}$ This cohort included 677 White children (355 boys and 322 girls) from southwestern Ohio who were measured via hand-wrist radiography on 13,823 occasions from 1932 to 1977 between the ages of 1 month and 22 years. ${ }^{39}$ For studies F0206S, F0375G, F0632G, and F0671G, radiographs taken to assess bone age were read centrally by the Fels Institute (now part of Lifespan Health Research Centre, Dayton, Ohio). Bone age assessments for Study 1419 were interpreted by individual investigators. Patients were considered to have attained near-adult height when the most recent bone age was at least 16 years for males and 14 years for females.

Among patients who achieved near-adult height, estimated improvement in near-adult height was a primary efficacy outcome. Improvement in near-adult height was estimated by comparing the mean gain in height among study patients with the expected change in height for age and sex in similar patients with untreated SPIGFD. The adult height that patients might have reached without mecasermin treatment was predicted using the Laron syndrome growth charts, which constitutes a reference range for patients with GHR abnormalities. ${ }^{18}$

\section{Height Standard Deviation Score}

Height SDS in patients naive to mecasermin treatment was a secondary efficacy outcome. Height SDS was computed using the US Centers for Disease Control and Prevention (CDC) age- and sex-dependent means and SDs. ${ }^{40}$ The CDC growth standards were developed with data collected in 5 cross-sectional, nationally representative surveys: National Household Education Survey II and (1963 to 65); National Household Education Survey III (1966 to 70); National Health and Nutrition Examination Survey (NHANES) (1971 to 74); NHANES II (1976 to 80 ); and NHANES III (1988 to 94). ${ }^{41}$ Some supplementary data sources were also used. ${ }^{41}$

\section{Skeletal Maturation}

\section{Bone Age Relative to Chronological Age}

Bone age relative to chronological age in patients naive to mecasermin treatment was a secondary efficacy outcome. As described previously, bone age was estimated via radiography of the hand and wrist using the Fels method. ${ }^{38}$ To assess the possible effect of mecasermin on the advancement of bone age, cumulative change in bone age was described relative to the cumulative change in chronological age over the same time period.

\section{Body Mass or Composition}

\section{Body Mass Index Standard Deviation Score}

The BMI SDS (also known as the BMI z score) was a secondary efficacy outcome. The CDC growth standards ${ }^{40}$ were used as a reference standard. As noted in the discussion of height SDS, the CDC growth standards are based on data collected between 1963 and 1980 from a nationally representative sample of males and females aged 2 to 20 years. ${ }^{40,41}$

\section{Statistical Analysis}

The sample sizes in the 4 predecessor studies were small due to the comparatively small size of the patient population and to the early nature of the studies. The sample sizes of all 5 studies were determined by convenience due to the rare nature of SPIGFD. The results were not adjusted for multiple comparisons.

Means, SDs, and ranges for baseline characteristics and treatment results were computed for patients who were naive to mecasermin treatment before enrolment in any of the 5 studies. 
Patients who received mecasermin for more than 2 weeks before enrolment, who did not have year 1 data, or who did not have pre-treatment height velocity data were discussed individually. One patient had a period of thrice-daily dosing, while another had a period of daily dosing. Dosages for these 2 patients were adjusted to twice daily in the analysis (i.e., the twice daily dosage used for the patient with daily dosing was half the daily dosage).

Height velocity during each year of treatment was compared with pre-treatment height velocity using 2-sided paired t-tests. Height velocity results were presented for each year of treatment, up to 19 years. Height velocity was also discussed in the context of historical results, as described by Laron et al. (1993)18 for patients with Laron syndrome. Similar analyses were presented for height velocity SDSs and height SDSs. Subgroup analyses were undertaken for age, etiology of GHIS, and the presence of antibodies, but these were not pre-specified.

Because time from baseline for the assessment of bone ages was not uniform across patients, a bivariate scatterplot was presented of the cumulative change in bone age versus the cumulative change in chronological age. Near-adult height was discussed in the context of historical results for untreated patients with Laron syndrome. ${ }^{18}$

Baseline serum IGF-1 concentration was taken as the IGF-1 concentration before the first dose of GH in the IGF-1 generation test. Levels of IGF-1 were measured in assays for which norms necessary for computing SDSs were not generally known. All IGF-1 SDSs were estimated using an algorithm derived from IGF-1 values obtained from a normative sample from Esoterix, Inc. The computation of expected mean and SD for age and sex were computed by the sponsor using these IGF-1 values as described by Frane and Bright (2004).42 Concentrations of IGF- 1 expressed as units per $\mathrm{mL}$ and $\mathrm{nmol} / \mathrm{L}$ were converted to $\mathrm{ng} / \mathrm{mL}$ by multiplying by 180 and 7.649, respectively. No IGF-1 data obtained in local assays for which no specific conversion to $\mathrm{ng} / \mathrm{mL}$ was possible were included in the data presentations or analyses. Concentrations of $\mathrm{GH}$ expressed as milli units or milli international units per litre were converted to $\mathrm{ng} / \mathrm{mL}$ by dividing by 3 .

The relationship of patient age and the occurrence of each notable harm was assessed using standard time-to-event analysis with censoring, with time to first occurrence as the dependent variable and the patient age at baseline as the independent variable. Two-sided t-tests were used to assess whether there was evidence that the harm in question was age-related. Subgroup analyses by age were pre-specified in the study protocol.

\section{Imputations for Missing Data}

Baseline Characteristics

When a baseline serum IGF-1 concentration was not available, the IGF-1 concentration from the pre-IGF-1 (trough) dose was used. When the IGF-1 levels were below the lower limit of detection or reported as "less than standard" (which sometimes occurs in patients with SPIGFD), the best estimate of the lower limit of detection was used.

Missing pubertal stages at baseline were imputed to be 1 when computing baseline characteristics for females up to 10.0 years and males up to 11.0 years of age. If the first non-missing pubertal age stage was 1 , then a missing baseline pubertal stage was imputed as 1 regardless of the baseline age when computing baseline characteristics. Whenever the pubertal stage at 1 clinic visit was stage 2 and was followed by 2 or more pubertal-stage values of stage 1, this pubertal stage of stage 2 was replaced by pubertal stage 1 . 


\section{Growth Outcomes}

Because height was not always measured at close to intervals of 1 year, annual heights were imputed by interpolation for computing annual heights, including baseline height. For any annual height, the interpolation was made between the closest date before the anniversary date and the closest date after the anniversary date. These interpolated heights were used in computing annual height velocities, height velocity SDSs, and height SDSs. If no height was measured after a given anniversary date, then the height from the closest date before the anniversary date was used in computing the annualized height velocity, height velocity SDS, and height SDS, provided that the prior date was within 90 days of the anniversary date in question. For some patients, neither a baseline height nor a height before baseline was recorded. For these patients, the first post-baseline height and corresponding date were used when computing the first-year annualized height velocity.

After initiating treatment, some patients temporarily discontinued mecasermin, either because they were in the placebo-controlled crossover study (F0375G) or for other reasons. For the purposes of computing pre-treatment height velocity and annual height velocity, baseline was considered as the time of the first injection of mecasermin and no accommodations or adjustments were made to height velocity for periods off treatment. For patients who received placebo during the first year in Study F0375G, the pre-treatment height velocity was equal to their height velocity during the placebo year.

When evaluating whether near-adult height had been attained, if bone age was not assessed at the last study visit, the bone age at the last study visit was imputed by assuming 1 bone age month advance for every chronological month advance since the last visit at which the bone age was assessed.

\section{Harms Outcomes}

The start and stop dates of some AEs were sometimes only partially known. Whenever the partial date implied the possibility that the event occurred either before or after the initiation of mecasermin treatment, it was assumed that the event took place after treatment initiation. When determining the treatment month and year of the occurrence of hypoglycemia, the missing day of the calendar day of the month in the date of the occurrence was imputed at 15 except when it would result in a calendar date before the start of treatment, in which case the date of occurrence was not imputed as the date of the first day of treatment. The date of occurrence was not imputed if the calendar month of hypoglycemia was not known. Hypoglycemic events reported as occurring before treatment start were excluded unless they were reported as possibly or probably related to treatment, in which case the month of the hypoglycemia was imputed as the first month of treatment. Imputation of the day of the month for time-to-event analyses for selected AEs followed the same imputation procedures.

\section{Analysis Populations}

Study 1419 reports on efficacy and safety data for all patients who were enrolled, either in Study 1419 or 1 of its predecessor studies.

\section{Results}

\section{Patient Disposition}

Ninety-one patients were enrolled in Study 1419 (Table 8). One patient who completed study F0671G did not enrol in Study 1419 but was included in the efficacy analysis in the integrated study report. Twenty-eight percent of patients $(n=26)$ had reached adult height and $9 \%(n=$ 
8) were still on treatment at the end of the study period. Nearly 2-thirds of patients ( $n=57$; $62 \%$ ) were discontinued from the study before the end of the study period, the majority of whom were lost to follow-up $(n=30 ; 33 \%)$ or transferred to the commercial drug $(n=14$; $15 \%)$. Few patients were discontinued due to noncompliance $(n=4 ; 4 \%)$ and there were no discontinuations due to AEs.

The primary efficacy analysis included all patients naive to mecasermin treatment before enrolment in any of the 5 studies who had been on treatment for at least 1 year $(n=81 ; 88 \%)$. The 92 patients enrolled who had received at least 1 dose of mecasermin includes all those who were naive to mecasermin upon entering the predecessor studies or Study 1419 ( $n=$ 83) and excludes those who were previously treated with Pharmacia mecasermin $(n=9)$ or for whom at least 1 year of treatment data were not available $(n=2)$. All patients enrolled in Study 1419 and/or any of the predecessor studies who had received at least 1 dose of mecasermin were included in the safety analysis $(n=92)$.

Table 8: Patient Disposition for Study 1419

\begin{tabular}{|c|c|}
\hline Patient disposition & Study 1419 \\
\hline Enrolled, N (\%) & $92^{\mathrm{a}}$ \\
\hline Attained near-adult height, $\mathrm{n}(\%)$ & $26(28)$ \\
\hline On treatment at end of study, $n$ (\%) & $8(9)$ \\
\hline Discontinued from study, $n$ (\%) & $57(62)$ \\
\hline \multicolumn{2}{|c|}{ Reason for discontinuation, $\mathrm{n}(\%)$} \\
\hline Adverse events & $0(0)$ \\
\hline Noncompliance & $4(4)$ \\
\hline Parent/patient decision & $2(2)$ \\
\hline Lost to follow-up ${ }^{b}$ & $30(33)$ \\
\hline Poor growth & $1(1)$ \\
\hline Patient transfer to commercial drug ${ }^{c}$ & $14(15)$ \\
\hline Unable to provide study drug ${ }^{d}$ & $6(7)$ \\
\hline Efficacy analysis, $\mathrm{N}^{\mathrm{e}}$ & 81 \\
\hline Safety analysis, $\mathbf{N}^{\mathrm{f}}$ & 92 \\
\hline
\end{tabular}

$\mathrm{SD}=$ standard deviation.

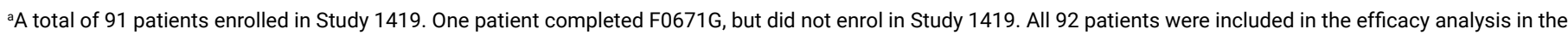
integrated study report for Study 1419 .

${ }^{b}$ Reasons for loss to follow-up were not described. These patients were had a mean age of 6.2 years $(S D=3.5)$ at baseline, and during a mean treatment period of 4.3 years $(S D=2.6)$, their mean height standard deviation scores increased from $-7.4(S D=1.7)$ to $-5.7(S D=2.1)$. The mean height velocity during the first year of

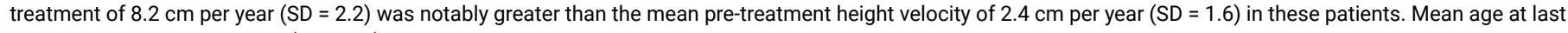
measurement was 10.5 years $(S D=4.7)$.

'Commercial drug not specified.

${ }^{\mathrm{d}}$ The patient moved to a country where mecasermin either could not be shipped to or could not be provided due to regulatory requirements.

eAll patients who were naive to mecasermin treatment and who had been on treatment for at least 1 year were included in the primary efficacy analysis.

${ }^{\mathrm{f}}$ All patients enrolled in Study 1419 and/or any of the predecessor studies who had received at least 1 dose of mecasermin were included in the safety analysis.

Source: Clinical Study Report for Study 1419.4 


\section{Exposure to Study Treatments}

All patients included in the primary efficacy analysis were naive to treatment with mecasermin and had been receiving treatment for at least 1 year. Studies to measure mecasermin concentrations in serum were not routinely performed to assess patients' compliance with the treatment regimen; however, just 4 patients (4\%) were discontinued due to noncompliance. The median duration of treatment was 5.5 years, while the mean was 6.0 years. Details of patients' exposure to mecasermin are provided in Table 9 and Figure 3. Most commonly, patients received $120 \mathrm{mcg} / \mathrm{kg}$ mecasermin SC twice daily (356 patient-years or $69 \%$ of a total 516 patient-years). Most of the rest of the exposure was at $80 \mathrm{mcg} / \mathrm{kg}$ mecasermin SC twice daily (50 patient-years or $10 \%$ of a total 516 patient-years).

\section{Efficacy}

Only those efficacy outcomes and analyses of subgroups identified in the review protocol are reported.

\section{Height}

Of the 81 patients who were naive to mecasermin treatment and completed at least 1 year of treatment, 75 had baseline height velocities and post-treatment height velocities and were included in the main analysis. Results for 9 patients who were not naive to mecasermin treatment were analyzed separately.

\section{Table 9: Exposure to Mecasermin}

\begin{tabular}{|l|c|}
\hline Twice-daily dose of mecasermin $(\mathrm{mcg} / \mathrm{kg})$ & Patient-years \\
\hline 30 & $<0.5$ \\
\hline 40 & 3 \\
\hline 50 & 1 \\
\hline 60 & 11 \\
\hline 70 & 4 \\
\hline 80 & 50 \\
\hline 90 & 7 \\
\hline 100 & 45 \\
\hline 110 & 22 \\
\hline 120 & 356 \\
\hline 130 & 5 \\
\hline 140 & 1 \\
\hline 150 & 9 \\
\hline 160 & 2 \\
\hline Total & 516 \\
\hline
\end{tabular}

Source: Clinical Study Report for Study $1419 .{ }^{15}$ 


\section{Height Velocity and Height Velocity Standard Deviation Score}

During year 1 of mecasermin treatment, there was an increase in mean height velocity from $2.6 \mathrm{~cm}$ per year $(S D=1.7)$ to $8.0 \mathrm{~cm}$ per year $(S D=2.3)(P<0.0001)$ (Table 10). Except for 7 patients (9\%), all had height velocities of last least $5.0 \mathrm{~cm}$ per year during the first year of treatment. Three of these patients had reached near-adult height. Height velocities for years 2 through 8 of treatment remained greater than those of baseline. There were positive trends for years 9,10 , and 11 , for which sample sizes were only 14,13 , and 12 , respectively.

Subgroup results presented in Study 1419 that were identified in the review protocol are reported. There was no correlation between age and year 1 height velocity (point estimates not reported; $P=0.50$ ). Results for patients who were naive to mecasermin treatment when they enrolled in Study 1419 (i.e., excluding patients who had enrolled in any of the other 4 predecessor studies, or had been previously treated with Pharmacia mecasermin; $n=52$ ) were similar to those in the primary efficacy analysis. The mean height velocities for the first 7 years of treatment for these patients were $8.2 \mathrm{~cm}$ per year, $6.0 \mathrm{~cm}$ per year, $5.8 \mathrm{~cm}$ per year, $5.4 \mathrm{~cm}$ per year, $5.1 \mathrm{~cm}$ per year, $4.8 \mathrm{~cm}$ per year, and $4.2 \mathrm{~cm}$ per year, respectively. The mean difference in year 1 height velocity was similar in patients with GH gene deletion $(n=$ $7 ; 7.4 \mathrm{~cm}$ per year $[S D=3.6])$ and patients with Laron syndrome phenotype $(n=72 ; 6.6 \mathrm{~cm}$ per year $[S D=3.8])(P=0.63)$. Serum samples were assayed for anti-IGF-1 antibodies in the first 4 studies: F0206S, F0375G, F0632G, and F0671G. Of the 23 patients for whom antibody titres were available in the first year of treatment, 12 were negative (mean age of 7.0 years [SD $=4.7$ ]; $75 \%$ male) and 11 were positive (mean age of 6.3 years [SD $=3.8$ ] years; $64 \%$ male). There was no difference in mean height velocity during the first year of treatment for those with antibodies compared to those without antibodies $(7.1 \mathrm{~cm}$ per year $[S D=3.0]$ versus 7.9 $\mathrm{cm}$ per year $[S D=2.1])(P=0.51)$.

There was an observed association between dose and height velocity during the first year of treatment. The mean year 1 height velocity at a dosage of up to $60 \mathrm{mcg} / \mathrm{kg} \mathrm{SC}$ twice daily

\section{Figure 3: Kaplan-Meier Plot of Duration of Mecasermin Therapy}

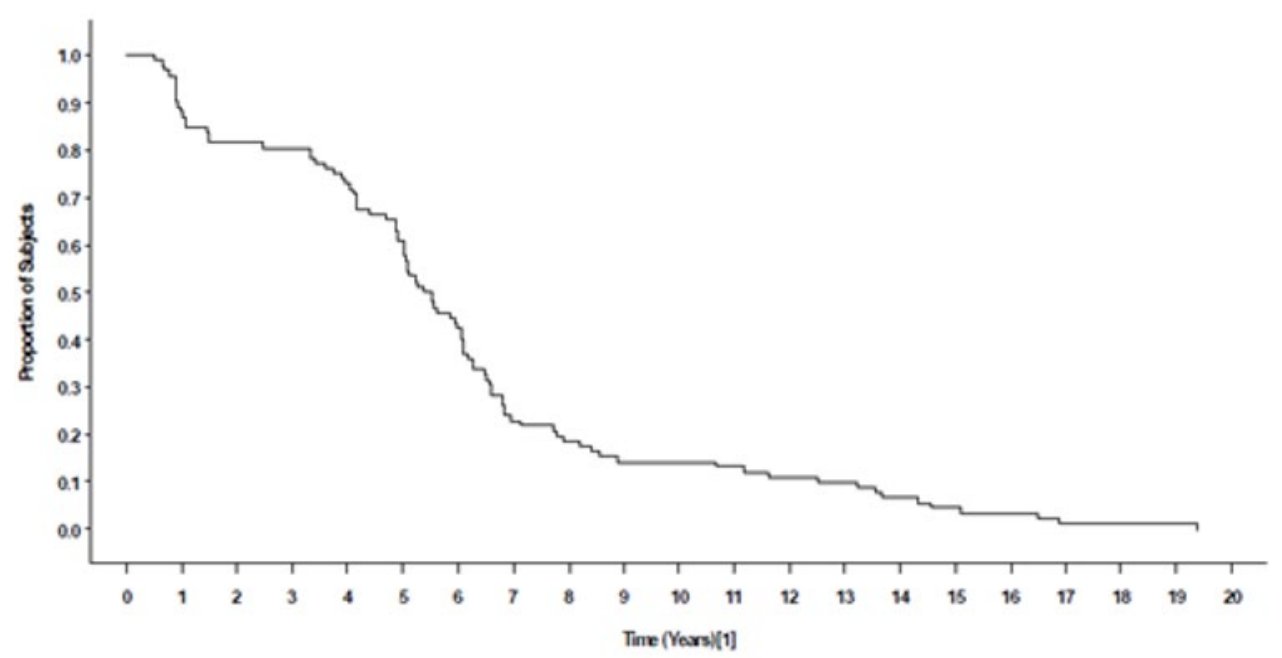

1] Nunber of years fromtine of first doee of IGF-I therapy to time of last height manourement. ban dersion of trecterext $=60$ Mbdin derstion of treaterent $=5.5$

IGF-1 = insulin-like growth factor 1 .

Source: Clinical Study Report for Study $1419 .{ }^{15}$ 
was $6.0 \mathrm{~cm}$ per year ( $95 \% \mathrm{Cl}, 5.1$ to 6.9$)$ compared with $8.5 \mathrm{~cm}$ per year $(95 \% \mathrm{Cl}, 7.8$ to 9.1$)$ at a dosage of $120 \mathrm{mcg} / \mathrm{kg} \mathrm{SC}$ twice daily $(P=0.0002)$ (Figure 4).

During the first year of mecasermin treatment, the mean height velocity SDS increased from $-3.4(S D=1.6)$ to $1.7(S D=2.8)(P<0.0001)$ (Table 11). The mean height velocity SDS for years 2 through 10 of treatment were also greater than baseline. Pre-treatment mean height velocity SDSs were similar for various numbers of years of treatment (range $=-4.4$ to -3.1 ) despite differences in the number of patients included in each analysis. Results for patients who were naive to mecasermin treatment when they were enrolled in Study 1419 were similar to those in the primary efficacy analysis.

\section{Near-Adult Height and Estimated Improvement in Near-Adult Height}

Over the course of the study, 19 patients naive to mecasermin achieved near-adult height based on bone age criteria (at least 16 years for males and 14 years for females). An additional 2 patients naive to mecasermin were considered by the investigators to have completed the intended course of treatment to near-adult height. The mean difference between observed and expected increase in height (from Laron et al. [1993]) ${ }^{18}$ was $13 \mathrm{~cm}$ $(\mathrm{SD}=8$; range $=-0.5$ to 35$)$ after an average of 11 years of treatment. The median final adult height was $137.6 \mathrm{~cm}$ (range $=112.0$ to 164.4 ).

Five patients who had previously been treated with Pharmacia mecasermin also achieved near-adult height. The long-term effect of mecasermin on near-adult height in these patients could not be evaluated due to a lack of detailed records during the period before enrolment in Study 1419. Sixty-one patients naive to mecasermin treatment terminated Study 1419 before attaining near-adult height and 1 patient completed Study F0671G but did not enrol in Study 1419. The first-year height velocity was greater than the pre-treatment height velocity in all 55 patients who had both pre-treatment and 1-year height velocities, except for 1 patient who was noncompliant. Similarly, height SDS increased in 57 of the 62 patients during the total course of treatment.

\section{Height Standard Deviation Score}

During the first year of mecasermin treatment, the mean height SDS increased from -6.9 $(S D=1.8)$ to $-6.1(S D=1.8)(P<0.0001)($ Table 12). The mean height SDS for years 2 through 14 of treatment were also greater than baseline. Pre-treatment mean height SDSs were similar for various numbers of years of treatment (range $=-6.9$ to -6.5 ) despite differences in the numbers of patients included in each analysis. There was a cumulative mean increase in height SDS of $1.8(S D=1.1)$. Results for patients who were naive to mecasermin treatment when they were enrolled in Study 1419 were similar to those in the overall analysis.

Twenty-one patients with limited growth potential at the commencement of puberty received leuprolide with the intention of delaying puberty to achieve a greater adult height by prolonging the growth period. Leuprolide together with mecasermin treatment appeared to increase the height SDS for $3(14 \%)$ of these patients, in whom the combination was used for a long period of time. Most other patients receiving leuprolide either had a marginal improvement in height SDS, or a decrease. Changes in height SDS for patients treated with leuprolide were not tested statistically due to the small sample size.

\section{Health-Related Quality of Life}

HRQoL was not reported in Study 1419 or its predecessors. 
Table 10: Height Velocity Among Patients Naive to Mecasermin in Study 1419

\begin{tabular}{|c|c|}
\hline Height velocity (cm per year) ${ }^{a}$ & Study 1419 \\
\hline Year 1 of treatment, $n$ & 75 \\
\hline Baseline height velocity, mean (SD) & $2.6(1.7)$ \\
\hline Height velocity during year 1 , mean (SD) & $8.0(2.3)$ \\
\hline Change from baseline height velocity, mean (SD) & $5.4(2.6)$ \\
\hline$P$ value ${ }^{b}$ & $<0.0001$ \\
\hline Year 2 of treatment, $n$ & 63 \\
\hline Baseline height velocity, mean (SD) & $2.7(1.8)$ \\
\hline Height velocity during year 2 , mean (SD) & $5.9(1.7)$ \\
\hline Change from baseline height velocity, mean (SD) & $3.2(2.6)$ \\
\hline$P$ value ${ }^{b}$ & $<0.0001$ \\
\hline Year 3 of treatment, $n$ & 62 \\
\hline Baseline height velocity, mean (SD) & $2.7(1.8)$ \\
\hline Height velocity during year 3 , mean (SD) & $5.5(1.8)$ \\
\hline Change from baseline height velocity, mean (SD) & $2.8(2.4)$ \\
\hline$P$ value ${ }^{b}$ & $<0.0001$ \\
\hline Year 4 of treatment, $n$ & 60 \\
\hline Baseline height velocity, mean (SD) & $2.6(1.8)$ \\
\hline Height velocity during year 4 , mean (SD) & $5.2(1.5)$ \\
\hline Change from baseline height velocity, mean (SD) & $2.5(2.5)$ \\
\hline$P$ value ${ }^{b}$ & $<0.0001$ \\
\hline Year 5 of treatment, $n$ & 53 \\
\hline Baseline height velocity, mean (SD) & $2.8(1.8)$ \\
\hline Height velocity during year 5 , mean (SD) & $4.9(1.5)$ \\
\hline Change from baseline height velocity, mean (SD) & $2.1(2.1)$ \\
\hline$P$ value ${ }^{b}$ & $<0.0001$ \\
\hline Year 6 of treatment, $n$ & 39 \\
\hline Baseline height velocity, mean (SD) & $2.9(1.9)$ \\
\hline Height velocity during year 6 , mean (SD) & $4.8(1.4)$ \\
\hline Change from baseline height velocity, mean (SD) & $1.9(2.1)$ \\
\hline$P$ value ${ }^{b}$ & $<0.0001$ \\
\hline Year 7 of treatment, $n$ & 25 \\
\hline Baseline height velocity, mean (SD) & $3.0(2.0)$ \\
\hline Height velocity during year 7 , mean (SD) & $4.3(1.5)$ \\
\hline
\end{tabular}




\begin{tabular}{|c|c|}
\hline Height velocity (cm per year) ${ }^{a}$ & Study 1419 \\
\hline Change from baseline height velocity, mean (SD) & $1.4(2.2)$ \\
\hline$P$ value $^{b}$ & 0.0042 \\
\hline Year 8 of treatment, $\mathrm{n}$ & 19 \\
\hline Baseline height velocity, mean (SD) & $3.1(2.2)$ \\
\hline Height velocity during year 8 , mean (SD) & $4.4(1.5)$ \\
\hline Change from baseline height velocity, mean (SD) & $1.3(2.8)$ \\
\hline$P$ value ${ }^{b}$ & 0.0486 \\
\hline Year 9 of treatment, $\mathrm{n}$ & 14 \\
\hline Baseline height velocity, mean (SD) & $3.3(2.3)$ \\
\hline Height velocity during year 9 , mean (SD) & $4.4(1.7)$ \\
\hline Change from baseline height velocity, mean (SD) & $1.1(3.2)$ \\
\hline$P$ value ${ }^{b}$ & 0.2061 \\
\hline Year 10 of treatment, $n$ & 13 \\
\hline Baseline height velocity, mean (SD) & $3.4(2.3)$ \\
\hline Height velocity during year 10 , mean (SD) & $4.5(2.0)$ \\
\hline Change from baseline height velocity, mean (SD) & $1.1(3.5)$ \\
\hline$P$ value ${ }^{b}$ & 0.2613 \\
\hline Year 11 of treatment, $n$ & 12 \\
\hline Baseline height velocity, mean (SD) & $3.5(2.4)$ \\
\hline Height velocity during year 11 , mean (SD) & $4.1(2.0)$ \\
\hline Change from baseline height velocity, mean (SD) & $0.7(3.3)$ \\
\hline$P$ value ${ }^{b}$ & 0.4879 \\
\hline Year 12 of treatment, $n$ & 10 \\
\hline Baseline height velocity, mean (SD) & $4.0(2.3)$ \\
\hline Height velocity during year 12 , mean (SD) & $3.9(2.0)$ \\
\hline Change from baseline height velocity, mean (SD) & $-0.1(3.2)$ \\
\hline$P$ value ${ }^{b}$ & 0.930 \\
\hline Year 13 of treatment, $n$ & 9 \\
\hline Baseline height velocity, mean (SD) & $3.5(2.0)$ \\
\hline Height velocity during year 13 , mean (SD) & $3.3(1.7)$ \\
\hline Change from baseline height velocity, mean (SD) & $-0.2(2.0)$ \\
\hline$P$ value ${ }^{b}$ & 0.7287 \\
\hline Year 14 of treatment, $\mathrm{n}$ & 6 \\
\hline Baseline height velocity, mean (SD) & $4.4(1.8)$ \\
\hline
\end{tabular}




\begin{tabular}{|l|c|}
\hline Height velocity (cm per year) $^{\mathrm{a}}$ & Study 1419 \\
\hline Height velocity during year 14, mean (SD) $^{\prime}$ & $2.3(1.6)$ \\
\hline Change from baseline height velocity, mean (SD) $^{\text {( }}$ & $-2.1(2.9)$ \\
\hline P value $^{\mathrm{b}}$ & 0.1372 \\
\hline
\end{tabular}

$\mathrm{SD}=$ standard deviation.

aResults are for 75 patients previously naive to mecasermin treatment who had completed at least 1 year of mecasermin treatment and who had baseline height velocities and post-treatment height velocities. Paired t-tests were used to compare the height velocities for a given year of treatment to the pre-treatment height velocities of the same patients completing that treatment year.

${ }^{\mathrm{b} P}$ value was not adjusted for multiple comparisons.

Source: Clinical Study Report for Study $1419 . .^{15}$

\section{Skeletal Maturation}

\section{Bone Age Relative to Chronological Age}

Of the 81 patients who were naive to mecasermin treatment, all who had a baseline bone age evaluation and a second bone age evaluation after at least 1 year of treatment but were not receiving concomitant treatment with leuprolide (to biochemically slow the advancement of bone age) were included in the main analysis $(n=56)$.

For patients with bone age measurements after at least 1 year of treatment $(n=56)$, bone age was delayed at baseline by a mean of 2.8 years $(S D=1.7)$ compared with chronological age (3.9 years [SD $=2.9$ ] versus 6.7 years [SD $=3.8]$, respectively) (Table 13). Change in bone age exceeded the change in chronological age during treatment by a mean 0.9 years $(S D=1.8)$ $(+7.4$ years $[S D=3.7]$ versus +6.5 years $[S D=3.7]$, respectively; $P=0.0004)$. The increment in bone age during mecasermin treatment was deemed not excessive relative to the increase in height, according to the investigators. Greater advances in bone age occurred during the first 5 years of treatment, when height velocity was the greatest (Figure 5). For patients with bone

\section{Figure 4: Year 1 Height Velocity Versus Dose of Mecasermin Per Injection in Study 1419}

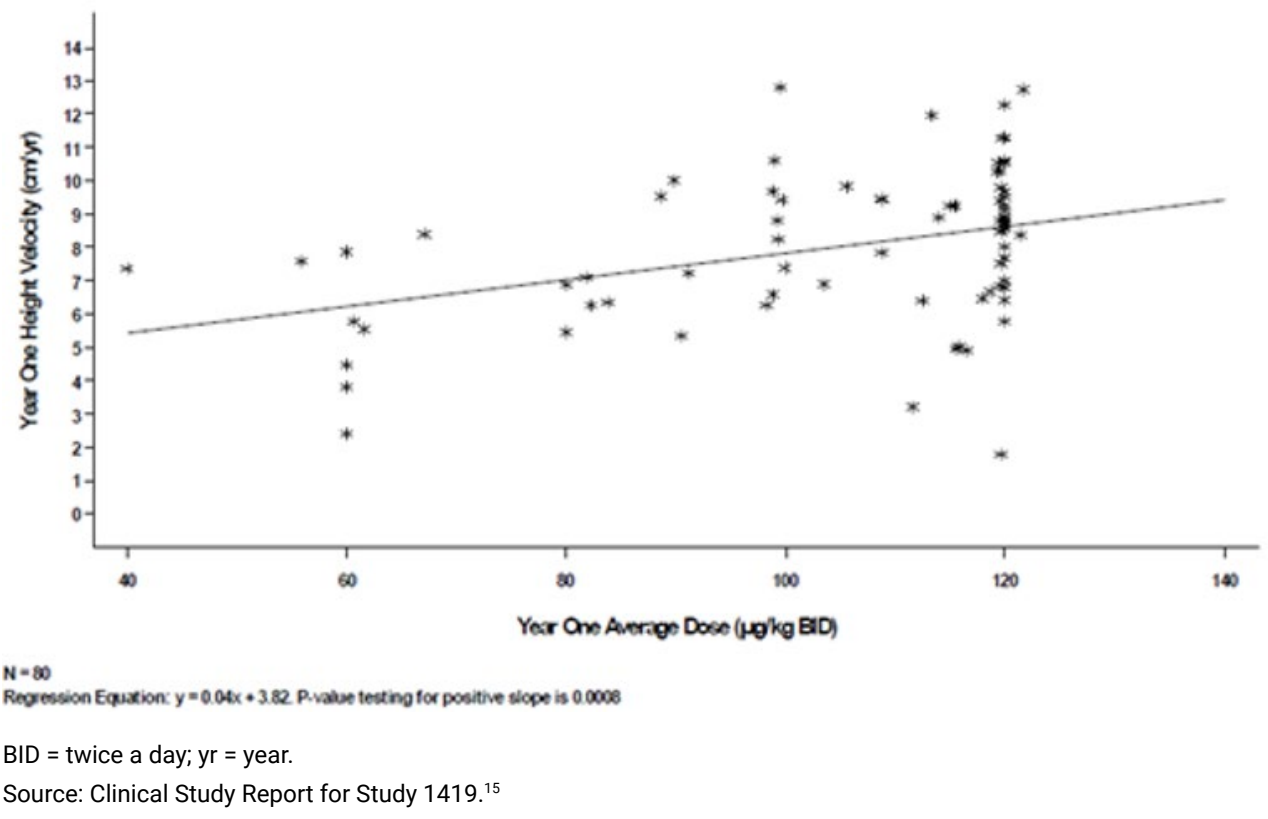


Table 11: Height Velocity SDS Among Patients Naive to Mecasermin in Study 1419

\begin{tabular}{|c|c|}
\hline Height velocity standard deviation score ${ }^{a}$ & Study 1419 \\
\hline Year 1 of treatment, $n$ & 75 \\
\hline Baseline height velocity SDS, mean (SD) & $-3.4(1.6)$ \\
\hline Height velocity SDS during year 1 , mean (SD) & $1.7(2.8)$ \\
\hline Change from baseline height velocity SDS, mean (SD) & $5.2(2.9)$ \\
\hline$P$ value ${ }^{b}$ & $<0.0001$ \\
\hline Year 2 of treatment, $n$ & 62 \\
\hline Baseline height velocity SDS, mean (SD) & $-3.4(1.6)$ \\
\hline Height velocity SDS during year 2 , mean (SD) & $-0.0(1.7)$ \\
\hline Change from baseline height velocity SDS, mean (SD) & $3.4(2.4)$ \\
\hline$P$ value ${ }^{b}$ & $<0.0001$ \\
\hline Year 3 of treatment, $\mathrm{n}$ & 61 \\
\hline Baseline height velocity SDS, mean (SD) & $-3.4(1.6)$ \\
\hline Height velocity SDS during year 3 , mean (SD) & $-0.1(1.9)$ \\
\hline Change from baseline height velocity SDS, mean (SD) & $3.3(2.3)$ \\
\hline$P$ value ${ }^{b}$ & $<0.0001$ \\
\hline Year 4 of treatment, $n$ & 58 \\
\hline Baseline height velocity SDS, mean (SD) & $-3.5(1.6)$ \\
\hline Height velocity SDS during year 4 , mean (SD) & $-0.2(1.9)$ \\
\hline Change from baseline height velocity SDS, mean (SD) & $3.2(2.4)$ \\
\hline$P$ value ${ }^{b}$ & $<0.0001$ \\
\hline Year 5 of treatment, $n$ & 50 \\
\hline Baseline height velocity SDS, mean (SD) & $-3.5(1.6)$ \\
\hline Height velocity SDS during year 5 , mean (SD) & $-0.3(1.7)$ \\
\hline Change from baseline height velocity SDS, mean (SD) & $3.2(2.1)$ \\
\hline$P$ value $^{b}$ & $<0.0001$ \\
\hline Year 6 of treatment, $n$ & 37 \\
\hline Baseline height velocity SDS, mean (SD) & $-3.5(1.6)$ \\
\hline Height velocity SDS during year 6 , mean (SD) & $-0.2(1.6)$ \\
\hline Change from baseline height velocity SDS, mean (SD) & $3.3(2.0)$ \\
\hline$P$ value $^{\mathrm{b}}$ & $<0.0001$ \\
\hline Year 7 of treatment, $n$ & 22 \\
\hline Baseline height velocity SDS, mean (SD) & $-3.5(1.7)$ \\
\hline Height velocity SDS during year 7 , mean (SD) & $-0.5(1.7)$ \\
\hline
\end{tabular}


Height velocity standard deviation score ${ }^{\mathrm{a}}$

Change from baseline height velocity SDS, mean (SD)

$P$ value ${ }^{b}$

Year 8 of treatment, $n$

Baseline height velocity SDS, mean (SD)

Height velocity SDS during year 8, mean (SD)

Change from baseline height velocity SDS, mean (SD)

$P$ value $^{\mathrm{b}}$

Year 9 of treatment, $\mathrm{n}$

Baseline height velocity SDS, mean (SD)

Height velocity SDS during year 9, mean (SD)

Change from baseline height velocity SDS, mean (SD)

$P$ value $^{b}$

Year 10 of treatment, $n$

Baseline height velocity SDS, mean (SD)

Height velocity SDS during year 10 , mean (SD)

Change from baseline height velocity SDS, mean (SD)

$P$ value ${ }^{b}$

Year 11 of treatment, $n$

Baseline height velocity SDS, mean (SD)

Height velocity SDS during year 11, mean (SD)

Change from baseline height velocity SDS, mean (SD)

$P$ value ${ }^{b}$

Year 12 of treatment, $n$

Baseline height velocity SDS, mean (SD)

Height velocity SDS during year 12 , mean (SD)

Change from baseline height velocity SDS, mean (SD)

$P$ value

Year 13 of treatment, $n$

Baseline height velocity SDS, mean (SD)

Height velocity SDS during year 13 , mean (SD)

Change from baseline height velocity SDS, mean (SD)

$P$ value ${ }^{b}$

Year 14 of treatment, $n$

Baseline height velocity SDS, mean (SD)

\section{Study 1419}

$3.0(2.1)$

$<0.0001$

15

$-3.6(1.9)$

$-0.2(1.6)$

$3.3(2.7)$

0.0003

12

$-3.6(2.0)$

$-0.4(0.8)$

3.2 (2.4)

0.0007

11

$-3.5(2.0)$

0.1 (1.6)

3.6 (3.0)

0.0026

11

$-3.5(2.0)$

$0.5(2.6)$

4.0 (3.9)

0.0072

8

$-3.5(1.3)$

$-0.1(1.5)$

3.5 (1.9)

0.0015

8

$-3.5(1.3)$

0.5 (2.1)

4.0 (2.3)

0.0016

5

$-3.1(1.3)$ 
Height velocity standard deviation score ${ }^{\mathrm{a}}$

Study 1419

\begin{tabular}{|l|c|}
\hline Height velocity SDS during year 14, mean (SD) & $-0.3(1.0)$ \\
\hline Change from baseline height velocity SD score, mean (SD) $^{-1.8}$ & $2.8(1.7)$ \\
\hline P value $^{\text {b }}$ & 0.0187 \\
\hline
\end{tabular}

$\mathrm{SD}=$ standard deviation; $\mathrm{SDS}$ = standard deviation score.

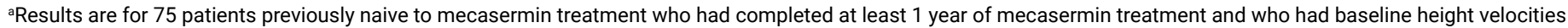
and post-treatment height velocities. Paired t-tests were used to compare the height velocity SDSs for a given year of treatment to the pre-treatment height velocity SDSs of the same patients completing that treatment year.

bP value was not adjusted for multiple comparisons.

Source: Clinical Study Report for Study $1419 .{ }^{15}$

ages measured after at least 6 years of treatment $(n=32)$, there was no advance of bone age relative to chronological age during mecasermin treatment $(+9.2$ years $[S D=3.6]$ versus +8.6 years $[S D=3.6]$, respectively, for a difference of 0.6 years $[S D=1.9])(P=0.0972)$.

\section{Body Mass or Composition}

BMI Standard Deviation Score

For the 81 patients with height and weight data, during the study there was a mean increase in BMI SDS from -0.3 $(S D=1.1)$ at baseline to $0.3(S D=1.4)$ when last evaluated, a difference of $0.6(S D=1.3)(P<0.0001)$. Most patients had a BMI SDS between -2 and +2 , both when first and last evaluated, or were nearly within this range or had little change.

\section{Harms}

Only those harms identified in the review protocol are reported. Table 14 provides detailed harms data.

\section{Adverse Events}

Seventy-six (83\%) patients had at least $1 \mathrm{AE}$ during treatment. The most reported AEs included metabolism and nutrition disorders $(n=48 ; 52 \%)$; general disorders and administration site conditions $(n=42 ; 46 \%)$; infections and infestations $(n=41 ; 45 \%)$; respiratory, thoracic, and mediastinal disorders $(n=38 ; 41 \%)$; gastrointestinal disorders $(n=$ $33 ; 36 \%)$; nervous system disorders $(n=31 ; 34 \%)$; and musculoskeletal and connective tissue disorders $(n=29 ; 32 \%)$.

\section{Serious Adverse Events}

Eighteen patients (20\%) had at least 1 SAE that required hospitalization. Adenoid hypertrophy occurred in 3 patients (3\%) and appendicitis occurred in 3 patients (3\%) (with bloody vomiting in 1 patients [1\%]). Other SAEs occurred in 1 patient (1\%) each: tonsillar hypertrophy; pneumonia with empyema; left radius fracture; enlarged adenoids; benign hypertension, enlarged tonsils, and slipped upper tibial epiphysis; abdominal pain and bacterial infection of the upper airway; tricuspid insufficiency and generalized seizure; ear-tube placement; hyperglycemia and possible diabetes mellitus; febrile seizure and skull fracture; renal calculus and apparent hypoglycemic seizures with loss of consciousness and flank pain with elevated minerals in urine; and bronchitis.

\section{Withdrawals Due to Adverse Events}

No patient withdrew from the study due to an AE. 
Table 12: Height SDS Among Patients Naive to Mecasermin Treatment in Study 1419

\begin{tabular}{|c|c|}
\hline Height standard deviation score ${ }^{a}$ & Study 1419 \\
\hline Year 1 of treatment, $n$ & 81 \\
\hline Baseline height SDS, mean (SD) & $-6.9(1.8)$ \\
\hline Height SDS during year 1 , mean (SD) & $-6.1(1.8)$ \\
\hline Change from baseline height SDS, mean (SD) & $0.8(0.6)$ \\
\hline$P$ value ${ }^{b}$ & $<0.0001$ \\
\hline Year 2 of treatment, $\mathrm{n}$ & 67 \\
\hline Baseline height SDS, mean (SD) & $-6.8(1.9)$ \\
\hline Height SDS during year 2, mean (SD) & $-5.6(1.7)$ \\
\hline Change from baseline height SDS, mean (SD) & $1.2(0.9)$ \\
\hline$P$ value & $<0.0001$ \\
\hline Year 3 of treatment, $n$ & 66 \\
\hline Baseline height SDS, mean (SD) & $-6.7(1.9)$ \\
\hline Height SDS during year 3 , mean (SD) & $-5.3(1.7)$ \\
\hline Change from baseline height SDS, mean (SD) & $1.4(1.1)$ \\
\hline$P$ value $^{b}$ & $<0.0001$ \\
\hline Year 4 of treatment, $n$ & 64 \\
\hline Baseline height SDS, mean (SD) & $-6.8(1.9)$ \\
\hline Height SDS during year 4 , mean (SD) & $-5.1(1.7)$ \\
\hline Change from baseline height SDS, mean (SD) & $1.6(1.2)$ \\
\hline$P$ value ${ }^{b}$ & $<0.0001$ \\
\hline Year 5 of treatment, $n$ & 57 \\
\hline Baseline height SDS, mean (SD) & $-6.7(1.8)$ \\
\hline Height SDS during year 5 , mean (SD) & $-5.0(1.7)$ \\
\hline Change from baseline height SDS, mean (SD) & $1.7(1.3)$ \\
\hline$P$ value ${ }^{b}$ & $<0.0001$ \\
\hline Year 6 of treatment, $n$ & -41 \\
\hline Baseline height SDS, mean (SD) & $-6.7(1.5)$ \\
\hline Height SDS during year 6 , mean (SD) & $-4.9(1.6)$ \\
\hline Change from baseline height SDS, mean (SD) & $1.8(1.1)$ \\
\hline$P$ value ${ }^{b}$ & $<0.0001$ \\
\hline Year 7 of treatment, $n$ & 26 \\
\hline Baseline height SDS, mean (SD) & $-6.6(1.4)$ \\
\hline Height SDS during year 7 , mean (SD) & $-4.9(1.7)$ \\
\hline
\end{tabular}


Height standard deviation score

Change from baseline height SDS, mean (SD)

$P$ value ${ }^{b}$

Year 8 of treatment, $n$

Baseline height SDS, mean (SD)

Height SDS during year 8, mean (SD)

Change from baseline height SDS, mean (SD)

$P$ value ${ }^{b}$

Year 9 of treatment, $n$

Baseline height SDS, mean (SD)

Height SDS during year 9, mean (SD)

Change from baseline height SDS, mean (SD)

$P$ value ${ }^{b}$

Year 10 of treatment, $n$

Baseline height SDS, mean (SD)

Height SDS during year 10, mean (SD)

Change from baseline height SDS, mean (SD)

$P$ value ${ }^{b}$

Year 11 of treatment, $n$

Baseline height SDS, mean (SD)

Height SDS during year 11, mean (SD)

Change from baseline height SDS, mean (SD)

$P$ value ${ }^{b}$

Year 12 of treatment, $\mathrm{n}$

Baseline height SDS, mean (SD)

Height SDS during year 12, mean (SD)

Change from baseline height SDS, mean (SD)

$P$ value $^{b}$

Year 13 of treatment, $n$

Baseline height SDS, mean (SD)

Height SDS during year 13, mean (SD)

Change from baseline height SDS, mean (SD)

$P$ value $^{\mathrm{b}}$

Year 14 of treatment, $\mathrm{n}$

Baseline height SDS, mean (SD)
Study 1419

$1.7(1.0)$

$<0.0001$

19

$-6.9(1.3)$

$-5.1(1.7)$

$1.7(1.0)$

$<0.0001$

14

$-6.8(1.5)$

$-5.0(1.6)$

$1.8(0.9)$

$<0.0001$

$-13$

$-6.8(1.5)$

$-5.0(1.7)$

1.9 (1.0)

$<0.0001$

12

$-6.6(1.3)$

$-4.7(1.2)$

$1.9(1.0)$

$<0.0001$

10

$-6.5(1.3)$

$-4.4(1.3)$

$2.0(0.9)$

$<0.0001$

9

$-6.8(0.7)$

$-4.7(1.0)$

$2.1(0.9)$

0.0001

6

$-6.7(0.6)$ 
Height standard deviation score ${ }^{\mathrm{a}}$

Study 1419

\begin{tabular}{|l|c|}
\hline Height SDS during year 14, mean (SD) & $-4.3(1.0)$ \\
\hline Change from baseline height SDS, mean (SD) $^{\text {(S. }}$ & $2.3(1.0)$ \\
\hline P value $^{\text {b }}$ & 0.0027 \\
\hline
\end{tabular}

SD = standard deviation; SDS = standard deviation score.

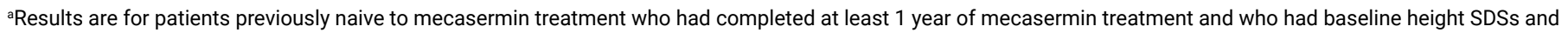
post-treatment height SDSs. Paired t-tests were used to compare the height SDSs for a given year of treatment to the pre-treatment height SDSs of the same patients completing that treatment year.

${ }^{\text {bP }}$ value was not adjusted for multiple comparisons.

Source: Clinical Study Report for Study $1419 .{ }^{15}$

\section{Mortality}

No patient died during the study.

\section{Notable Harms}

The most frequently reported notable harms were hypoglycemia ( $n=43 ; 47 \%)$; lipohypertrophy at the injection site $(n=32 ; 35 \%)$; tonsillar hypertrophy $(n=19 ; 21 \%)$; and adenoidal hypertrophy $(n=9 ; 10 \%)$.

\section{Critical Appraisal Internal Validity}

Study 1419 was a non-randomized, single-arm trial, open-label trial. Participants served as their own controls, whereby growth outcomes were continuously monitored during treatment and compared to baseline values via paired analyses. The only exception was for final adult height among patients who achieved adult height, for whom historical controls (patients with untreated Laron syndrome) were used to estimate the improvement in adult height. Due to the rare and severe nature of SPIGFD, a randomized control group was not feasible for the primary efficacy outcomes, and the findings are therefore at high risk of confounding. The investigators did not control for known prognostic factors or effect modifiers (e.g., environmental or socioeconomic factors). Because there was no control group for most analyses (with the exception of final adult height, which used historical controls), no strong conclusions can be made regarding the benefits and harms of treatment. The conclusions are also limited because the analyses were not adjusted for multiple comparisons, increasing the risk of a type I error.

It is unclear from the study protocol and Clinical Study Report how patients were selected for enrolment, creating the potential for selection bias (i.e., selection of patients who would most likely benefit from the treatment) that could overestimate the treatment effect. Specifically, 16 patients (19\%) included in the primary efficacy analyses did not fully meet the eligibility criteria (e.g., they did not have an IGF-1 SDS of less than -2), and the mean effect could therefore be different than that observed in patients who meet all criteria for SPIGFD. The eligibility criteria may also have excluded patients without any genetic defect who would still meet the clinical criteria for SPIGFD. The sample size was based on convenience given the rare nature of the disease, and no power calculations were performed. The study may have been underpowered to detect statistically significant changes in outcomes, particularly at later time points when fewer patients remained in the study (e.g., after 8 years of treatment when fewer than 15 patients had measurements available for change in height velocity). 
For the estimated improvement in adult height, historical controls were used. The historical control group was a cohort of 24 patients (10 males and 14 females) with Laron syndrome who were followed from infancy to adulthood at a single clinic in Israel..$^{18}$ The Laron growth

\section{Table 13: Change in Bone Age Relative to Chronological Age for Patients Naive to Mecasermin Treatment Who Were Not Taking Leuprolide in Study 1419}

\section{Bone age relative to chronological age (years)}

\section{Study 1419}

Patients with bone age measurements after at least 1 year

\begin{tabular}{|c|c|}
\hline Number of patients analyzed & 56 \\
\hline Baseline bone age, mean (SD) & $3.9(2.9)$ \\
\hline Baseline chronological age, mean (SD) & $6.7(3.8)$ \\
\hline Baseline bone age delay, mean (SD) & $2.8(1.7)$ \\
\hline Last bone age, mean (SD) & $11.3(\mathrm{NA})$ \\
\hline Last chronological age, mean (SD) & $13.2(\mathrm{NA})$ \\
\hline Last bone age delay, mean (SD) & $1.9(\mathrm{NA})$ \\
\hline Change from baseline bone age, mean (SD) & $7.4(3.7)$ \\
\hline Change from baseline chronological age, mean (SD) & $6.5(3.7)$ \\
\hline $\begin{array}{l}\text { Difference of change in bone age and change in chronological } \\
\text { age, mean (SD) }\end{array}$ & $0.9(1.8)$ \\
\hline$P$ value ${ }^{b}$ & 0.0004 \\
\hline \multicolumn{2}{|c|}{ Patients with bone age measurements after at least 6 years ${ }^{c}$} \\
\hline Number of patients analyzed & 32 \\
\hline Baseline bone age, mean (SD) & $3.8(2.6)$ \\
\hline Baseline chronological age, mean (SD) & $6.3(3.4)$ \\
\hline Baseline bone age delay, mean & $2.5(1.5)$ \\
\hline Last bone age, mean (SD) & $13.0(\mathrm{NA})$ \\
\hline Last chronological age, mean (SD) & 14.9 (NA) \\
\hline Last bone age delay, mean (SD) & $1.9(\mathrm{NA})$ \\
\hline Change from baseline bone age, mean (SD) & $9.2(3.6)$ \\
\hline Change from baseline chronological age, mean (SD) & $8.6(3.6)$ \\
\hline $\begin{array}{l}\text { Difference of change in bone age and change in chronological } \\
\text { age, mean (SD) }\end{array}$ & $0.6(1.9)$ \\
\hline$P$ value ${ }^{b}$ & 0.0972 \\
\hline
\end{tabular}

$\mathrm{NA}=$ not applicable; $\mathrm{SD}=$ standard deviation.

aResults are for patients naive to mecasermin treatment with a baseline bone age evaluation and a second bone age evaluation after at least 1 year of treatment who were not receiving concomitant treatment with leuprolide.

${ }^{\mathrm{b} P}$ value was not adjusted for multiple comparisons.

${ }^{\mathrm{c}}$ Results are for patients naive to mecasermin treatment with a baseline bone age evaluation and a second bone age evaluation after at least 6 years of treatment who were not receiving concomitant treatment with leuprolide.

Source: Clinical Study Report for Study $1419 .{ }^{15}$ 
curves are the only available comparator for final adult height among untreated patients, but their validity as a comparator in Study 1419 is uncertain. There is a risk that the estimated improvement in adult height could be biased due to differences in baseline characteristics of patients in Study 1419 compared to those studied by Laron et al. (1993). Most notably, the etiology of SPIGFD in Study 1419 was not Laron syndrome in all patients and the patient population was multinational. Further, there are no data to determine whether the final adult height in untreated patients has changed since the time of data collection by Laron et al. nearly 30 years ago. Between 1989 and 2019, final adult height among healthy individuals increased in many countries, particularly in emerging economies (for a maximum difference of up to $+7 \mathrm{~cm}$ in girls and $+9 \mathrm{~cm}$ in boys, although there was a large variation across countries). ${ }^{43}$ To our knowledge, no data are available on how the final adult height of patients with untreated SPIGFD may have changed (if at all) since 1993.

SDSs were calculated in Study 1419 using growth curves designed for the general population, either in the US (for height and BMI) or the UK (for height velocity). These need to be interpreted in context, as the results could have differed depending on the reference standard used. Specifically, the age- and sex-dependent means and SDs provided by Tanner et al. (1966) ${ }^{35,36}$ for height velocity were developed using a cohort of primarily normally growing, White, British children. Their applicability to diverse populations of children has been criticized. ${ }^{37}$ As children begin to enter puberty at earlier ages, the timing of peak height velocity has shifted, and it could be argued that the Tanner et al. (1966) standards are no longer applicable to contemporary children. ${ }^{37}$ The CDC growth charts used in the US for height SDS and BMI SDS calculations were developed using 5 cross-sectional, nationally representative health-examination surveys and are considered generalizable to all children in the US. ${ }^{41}$ The CDC growth charts, published in 2000, ${ }^{40}$ were the predominant growth charts used in Canada until the adoption of WHO child growth standards in $2007^{44}$ (and WHO growth charts adapted for Canada in 2010 and updated in 2014). ${ }^{45}$

\section{Figure 5: Cumulative Change in Bone Age Versus Cumulative Change in Chronological Age for Patients Naive to Mecasermin Treatment in Study 1419}

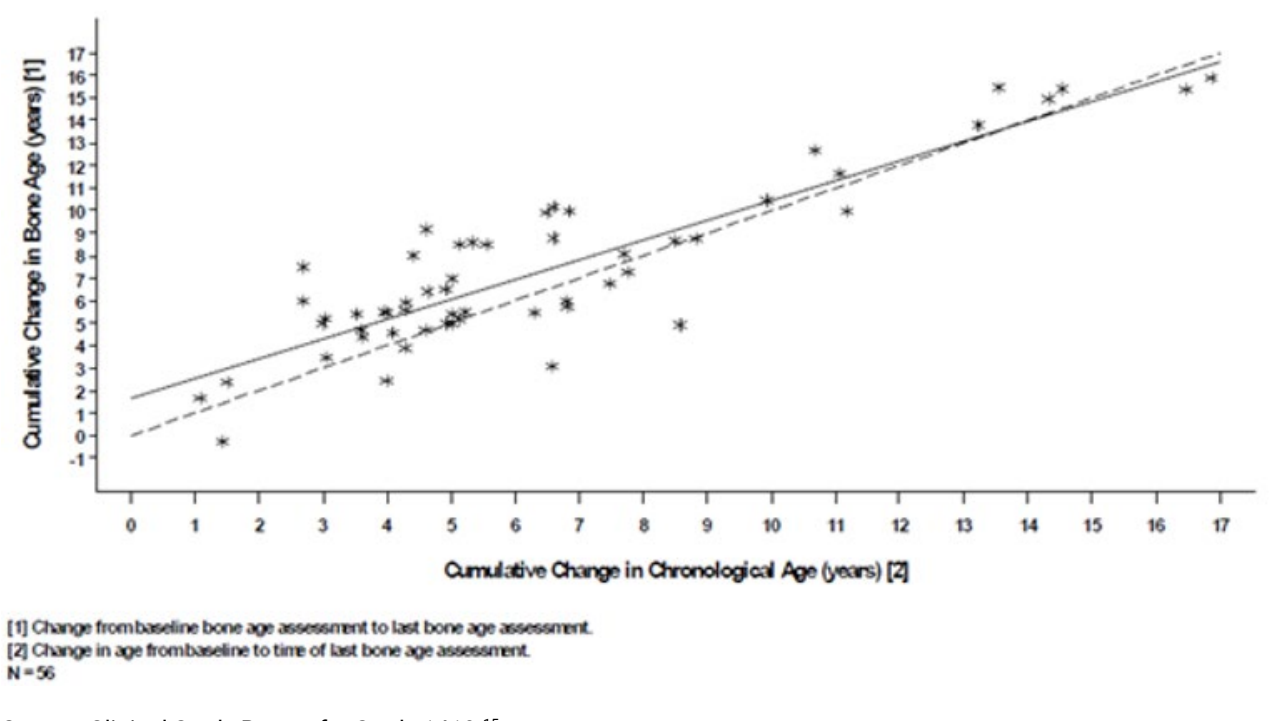

Source: Clinical Study Report for Study $1419 .{ }^{15}$ 
Table 14: Summary of Harms for Study 1419

\begin{tabular}{|c|c|}
\hline Harms & Study $1419(\mathrm{~N}=92)$ \\
\hline \multicolumn{2}{|c|}{ Patients with $\geq 1$ adverse event } \\
\hline$n(\%)$ & $76(83)$ \\
\hline \multicolumn{2}{|l|}{ Most common events, ${ }^{a} \mathrm{n}(\%)$} \\
\hline Metabolism and nutrition disorders & $48(52)$ \\
\hline General disorders and administration site conditions & $42(46)$ \\
\hline Infections and infestations & $41(45)$ \\
\hline Respiratory, thoracic, and mediastinal disorders & $38(41)$ \\
\hline Gastrointestinal disorders & $33(36)$ \\
\hline Nervous system disorders & $31(34)$ \\
\hline Musculoskeletal and connective tissue disorders & $29(32)$ \\
\hline Skin and subcutaneous tissue disorders & $26(28)$ \\
\hline Ear and labyrinth disorders & $25(27)$ \\
\hline Investigations & $24(26)$ \\
\hline Blood and lymphatic system disorders & $20(22)$ \\
\hline Eye disorders & $20(22)$ \\
\hline Injury, poisoning, and procedural complications & $15(16)$ \\
\hline Cardiac disorders & $14(15)$ \\
\hline Psychiatric disorders & $13(14)$ \\
\hline Reproductive system and breast disorders & $13(14)$ \\
\hline Surgical and medical procedures & $12(13)$ \\
\hline \multicolumn{2}{|c|}{ Patients with $\geq 1$ serious adverse event } \\
\hline $\mathrm{n}(\%)$ & $18(20)$ \\
\hline \multicolumn{2}{|l|}{ Most common events ${ }^{\mathrm{b}}, \mathrm{n}(\%)$} \\
\hline Adenoid hypertrophy & $3(3)$ \\
\hline Appendicitis & $3(3)$ \\
\hline \multicolumn{2}{|c|}{ Patients who stopped treatment due to adverse events } \\
\hline$n(\%)$ & $0(0)$ \\
\hline \multicolumn{2}{|c|}{ Deaths } \\
\hline$n(\%)$ & $0(0)$ \\
\hline \multicolumn{2}{|c|}{ Notable harms } \\
\hline Hypoglycemia, n (\%) & $43(47)$ \\
\hline Lipohypertrophy at the injection site, $\mathrm{n}(\%)$ & $32(35)$ \\
\hline Tonsillar hypertrophy, $\mathrm{n}(\%)$ & $19(21)$ \\
\hline
\end{tabular}




\begin{tabular}{|l|c|}
\hline Adenoidal hypertrophy, $\mathrm{n}(\%)$ & $9(10)$ \\
\hline Arthralgia, $\mathrm{n}(\%)$ & $8(9)$ \\
\hline $\begin{array}{l}\text { Benign, malignant, and unspecified neoplasms, including cysts and } \\
\text { polyps, } \mathrm{n}(\%)\end{array}$ & $7(8)$ \\
\hline Hypoglycemic seizure or convulsion, $\mathrm{n}(\%)$ & $7(8)$ \\
\hline Benign intracranial hypertension, $\mathrm{n}(\%)$ & $6(7)$ \\
\hline Myalgia, $\mathrm{n}(\%)$ & $2(2)$ \\
\hline Nephrolithiasis, $\mathrm{n}(\%)$ & $2(2)$ \\
\hline
\end{tabular}

${ }^{a}$ Frequency greater than $10 \%$. Common adverse events are summarized by the Medical Dictionary for Regulatory Activities system organ class for patients who received at least 1 dose of mecasermin.

${ }^{\mathrm{b}}$ Events that occurred in more than 1 patient. Source: Clinical Study Report for Study $1419 .{ }^{15}$

Because the trial was open-label, all patients, their parents or caregivers, the treating clinicians, and the outcome assessors were aware of the treatment assignment. Performance bias is always possible in open-label trials because patients and/or their caregivers who know the intervention to which they were assigned may perceive or detect an enhanced treatment effect. Detection bias is also possible because the outcome assessors may exaggerate treatment effects if they are aware of the treatment. With respect to the efficacy outcomes in Study 1419, the risk of both performance and detection bias are low because they were objectively measured. There is some risk that common subjective harms known to be associated with mecasermin could have been over-reported because patients and their treating clinicians were aware of the treatment received and of their participation in a trial.

All outcomes outlined in the study protocol were included in the Clinical Study Report, and the risk of bias due to selective reporting is low. Results were presented for all participants who were enrolled. A large proportion of patients $(62 \%)$ discontinued treatment early, and more than half (33\%) of those were lost to follow-up before attaining near-adult height. There is a high risk that the long-term efficacy outcomes and harms data could be biased due to missing outcomes for these patients (although the direction of the bias is unclear). Nevertheless, the efficacy outcomes at last follow-up for these patients were similar to those of the remaining study population, and no patient withdrew due to AEs. Imputations for pubertal stage at baseline appear reasonable, and imputations of annual height (for computing height velocity, height velocity SDS, and height SDS when data for height were missing) and bone age appear conservative (i.e., they would not be expected to have overestimated the treatment effect). The assumption that AEs occurred after the initiation of treatment when the start and stop dates of AEs were unknown was cautious and could have resulted in an overestimate of AEs during treatment.

\section{External Validity}

Study 1419 included an international group of patients with SPIGFD, with eligibility criteria that allowed for patients with less-severe short stature than that described in the product monograph (i.e., a height SDS of -2 compared with less than -3 in the product monograph). ${ }^{12}$ Because only 1 patient had a baseline height SDS of greater then -3 , the patient population is closely reflective of eligible Canadian patients based on height alone. Sixteen of the patients included in the primary growth outcome analyses did not meet all eligibility criteria (e.g., did 
not have an IGF-1 SDS of less than -2); however, all patients had genetically proven SPIGFD. Based on the eligibility criteria, some patients without a genetic cause of SPIGFD may have been excluded, and it is uncertain whether the results can be extrapolated to this group of patients. Although the condition affects males and females equally, there was an imbalance in the proportion of males and females enrolled in the study ( $58 \%$ male). This is unlikely to severely affect the generalizability of the findings; according to the clinical expert consulted for this review, the treatment is expected to have an equivalent effect in males and females (although their growth trajectories may differ).

The exposure to mecasermin in Study 1419 is likely reflective of typical exposures for patients who would be treated in the Canadian context. For the most part, patients in Study 1419 were treated with doses of mecasermin as recommended in the product monograph (80 $\mathrm{mcg} / \mathrm{kg}$ to $120 \mathrm{mcg} / \mathrm{kg} \mathrm{SC}$ twice daily) ${ }^{12}$; however, some pubertal patients were treated with doses up to $160 \mathrm{mcg} / \mathrm{kg}$ SC twice daily $(40 \mathrm{mcg} / \mathrm{kg} \mathrm{SC}$ twice daily more than recommended in the product monograph). The few exposures outside of the recommended dosage are aligned with expected clinical practice in Canada, where dosages would be prescribed on a case-by-case basis based on treatment response and tolerability. Patients in Study 1419 were exposed to mecasermin for 6 years on average. The time on mecasermin is reflective of the age at the start of treatment, which was highly variable.

Twenty-one patients were treated with leuprolide to prolong the growth period in Study 1419. Although leuprolide is not approved for this indication in Canada, some physicians may choose to use leuprolide in conjunction with mecasermin treatment for this purpose. This would be decided on a case-by-case basis for some children who may experience some benefit (namely, for those close to near-adult height or for whom bone age is increasing rapidly). The small proportion of patients treated with leuprolide concomitant to mecasermin in this study is therefore aligned with what would occur in the Canadian context and should not negatively affect the generalizability of the findings.

\section{Other Relevant Evidence}

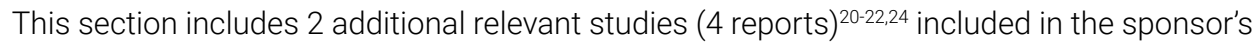
submission to CADTH. These were considered to address important gaps in the evidence included in the systematic review.

\section{Real-World Evidence: The European Increlex Growth Forum Database Registry}

Three reports ( 2 sponsor-submitted reports and 1 identified via the search) ${ }^{20-22}$ of 1 registry study were included to provide real-world evidence for the use of mecasermin in the treatment of children and adolescents with SPIGFD. Aside from providing long-term evidence on the efficacy and safety of mecasermin treatment in a representative sample of the treatment population, the European Increlex Growth Forum Database (EU-IGFD) Registry study fills an important evidence gap by providing information about treatment compliance and long-term safety after the discontinuation of treatment.

\section{Methods}

Implemented by Ipsen Pharma SAS in December 2008 in 10 European countries, the EU-IGFD Registry is a descriptive, multi-centre, observational, prospective, open-ended, noninterventional, post-authorization safety surveillance study of mecasermin. The primary objective of the EU-IGFD Registry is to collect long-term safety information on the use of mecasermin for the treatment of children with growth failure. Secondary objectives are to: 
(a) obtain long-term growth data for mecasermin therapy in children with growth failure by the evaluation of changes from baseline for height, height velocity, timing and progression of puberty, bone age development, dosing compliance, and IGF-1 levels; (b) model height velocity, predicted adult height, final adult height, timing and progression of puberty, and bone age development; (c) evaluate mecasermin treatment exposure and compliance; and (d) collect safety information 2 and 5 years after the end of mecasermin therapy in patients exposed for at least 3 years irrespective of their final height.

The study aims to recruit as many patients as possible at participating sites to obtain a sample that is representative of the treated population. Patients with growth failure for whom mecasermin treatment was indicated and who were initiating or already receiving mecasermin therapy were enrolled in the study and followed throughout their course of treatment. The decision to prescribe mecasermin was made before, and independently from, the decision to enrol patients in the study. Investigators were free to choose the dose and administration schedule, which was tailored to each patient. Study follow-up visits followed routine medical practices, and data were collected until adult height was achieved. The treatment duration and timing of clinical assessments were at the discretion of the investigator according to their judgment based on the clinical needs of the patient. Patients exposed to mecasermin for at least 3 years were asked to perform a post-treatment safety visit at 2 and 5 years, irrespective of final height.

\section{Populations}

\section{Inclusion and Exclusion Criteria}

Patients were eligible for participation if: (a) they were beginning therapy with mecasermin for growth retardation or were previously treated with mecasermin prescribed by a participating qualified practitioner; and (b) their parents or legally authorized representatives gave signed informed consent, and they provided assent, as appropriate. Patients were excluded if they were currently participating in a clinical trial for mecasermin or growth retardation. Patients were withdrawn if informed consent was withdrawn; they attained adult height; they were lost to follow-up; they were included in a clinical trial for growth retardation; or if the treating physician had changed (the patient could be included again by another physician).

\section{Baseline Characteristics}

Baseline characteristics for the EU-IGFD Registry are presented for 281 patients enrolled from 10 countries in Europe (Austria, Belgium, France, Germany, Italy, The Netherlands, Poland, Spain, Sweden, and the UK) as of May 13, 2019 (Table 15). Of these patients, 275 who had taken mecasermin at least once and completed at least 1 follow-up visit were included in the efficacy analysis.

The mean chronological age of patients at baseline was 9.5 years $(S D=4.1)$ years, with a range of 0.4 to 19.1 years. Mean bone age was $8.6(S D=3.5)$ years. More than half of patients were male $(n=177 ; 63 \%)$, and most patients did not have Laron syndrome $(n=$ 238; 85\%). Most patients began treatment at pubertal stage $1(n=225 ; 80 \%)$. Few $(n=24$; $9 \%)$ had received prior IGF-1 therapy. About one-quarter $(n=73 ; 26 \%)$ had received prior therapy with $\mathrm{GH}$.

All patients had severe short stature, with mean height and height SDS of $114.3 \mathrm{~cm}$ (SD = 21.4) and -3.8 (SD =1.3), respectively. Mean pre-treatment height velocity was $\mathrm{cm}$ per year $4.7(S D=1.7)$. Patients started treatment at a mean body weight and body weight SDS of 22.0 
$\mathrm{kg}(\mathrm{SD}=9.8)$ and $-3.3(\mathrm{SD}=1.4)$, respectively. The mean BMI and BMI SDS at baseline were $16.1 \mathrm{~kg} / \mathrm{m}^{2}(\mathrm{SD}=2.9)$ and $-0.7(\mathrm{SD}=1.4)$, respectively.

The mean serum IGF-1 for treatment-naive or prepubertal patients was $83.2 \mathrm{ng} / \mathrm{mL}$ (SD = 64.5 ) and for previously treated or pubertal patients it was $167.4 \mathrm{ng} / \mathrm{mL}(\mathrm{SD}=160.3)$. The mean maximum GH level for treatment-naive or prepubertal patients was $26.6 \mathrm{ng} / \mathrm{mL}$ $(S D=24.6)$ and for previously treated or pubertal patients it was $28.9 \mathrm{ng} / \mathrm{mL}(\mathrm{SD}=49.7)$, respectively.

\section{Interventions}

All patients received mecasermin. There was no control group and the only comparator was within-patient change from baseline. The mecasermin $10 \mathrm{mg} / \mathrm{mL}$ solution was packaged and presented according to the approved marketing authorization in each country. Mecasermin was administered via SC injection by the patient or their caregiver with the injection site changed with each injection. The recommended starting dosage was $0.04 \mathrm{mg} / \mathrm{kg} \mathrm{SC}$ twice daily and the maximum dosage was $0.12 \mathrm{mg} / \mathrm{kg}$ SC twice daily. Doses of mecasermin, changes, interruption periods and number of missed doses were recorded at each visit; however, information on treatment compliance was not collected. The study was designed to document current clinical practice, and investigators were permitted to alter or initiate concomitant medication(s) based on each patient's clinical need.

\section{Outcome Measures}

Efficacy and safety data were collected by investigators via an electronic CRF. Staff at each participating centre were trained by sponsor representatives and provided user manuals for data entry. Real-time checks were running at the time of data entry in the electronic CRF to prevent data entry errors and to ensure global consistency. The data were also reviewed by a data-management group for completeness, consistency, legibility, and protocol compliance. Data were monitored remotely and interactive queries were raised to clarify data further when needed. The sponsor assigned monitors to conduct site visits, and the investigator had to allow direct access to all relevant files for verifying entries made in the electronic CRF. The monitor compared the source documents to electronic CRF entries for accuracy.

\section{Efficacy Outcomes}

Growth outcome measurements were taken during usual visits, including height and height SDS; height velocity; weight and weight SDS; BMI and BMI SDS; bone age; predicted adult height; and final adult height. Measurement of HRQoL used the Pediatric Quality of Life Inventory (France only), which was completed by the patients, a parent, or caregiver. The timing of the follow-up visits was in line with current practice at the department or service.

For France and southern European countries, height and weight SDS were calculated using Sempé reference means and SD age- and sex-dependent values. ${ }^{46}$ The BMI SDSs were calculated using the French National Plan for Nutrition and Health reference means and SD values. ${ }^{47}$ For the UK, Belgium, Sweden, and Poland, height, weight, and BMI SDSs were calculated using UK reference values (although it is unclear which of the available reference values in use in the $\mathrm{UK}^{48}$ were leveraged in this study).

\section{Harms}

Safety assessments included AEs, laboratory test results, and/or vital sign data. Investigators were asked to report all SAEs (related or not) and all related non-serious AEs. Any clinically significant laboratory abnormality had to be reported as an $A E$, regardless of whether it was 
Table 15: Summary of Baseline Characteristics for EU-IGFD Registry

\begin{tabular}{|c|c|}
\hline Characteristic & EU-IGFD Registry $\left(\mathrm{N}=281^{\mathrm{a}}\right)$ \\
\hline Pre-treatment age (years), mean (SD) & $9.5(4.1)$ \\
\hline Median (minimum to maximum) & $9.6(0.4$ to 19.1$)$ \\
\hline \multicolumn{2}{|c|}{ Sex, n (\%) } \\
\hline Male & $177(63)$ \\
\hline Female & $104(37)$ \\
\hline \multicolumn{2}{|c|}{ Etiology of GHIS, n (\%) } \\
\hline Laron syndrome & $43(15)$ \\
\hline Other (not Laron syndrome) & $238(85)$ \\
\hline \multicolumn{2}{|c|}{ Pubertal stage, $n(\%)$} \\
\hline 1 & $225(80)$ \\
\hline 2 & $26(9)$ \\
\hline 3 & $14(5)$ \\
\hline 4 & $3(1)$ \\
\hline 5 & $2(1)$ \\
\hline Unknown & $11(4)$ \\
\hline \multicolumn{2}{|c|}{ Previous growth therapy, n (\%) } \\
\hline IGF-1 therapy & $24(9)$ \\
\hline Steroids & $5(2)$ \\
\hline GH therapy & $73(26)$ \\
\hline \multicolumn{2}{|l|}{ Enrolment IGF-1 (ng/mL), mean (SD) } \\
\hline Naive prepubertal patients & $83.2(64.5)$ \\
\hline Pubertal or previously treated patients & $167.4(160.3)$ \\
\hline \multicolumn{2}{|c|}{ Enrolment IGF-1 $(\mathrm{ng} / \mathrm{mL}$ ), median (minimum to maximum) } \\
\hline Naive prepubertal patients & $66.1(25.0$ to 384.0$)$ \\
\hline Pubertal or previously treated patients & 114.0 (25.0 to 859.3$)$ \\
\hline Pre-treatment height $(\mathrm{cm})$, mean $(\mathrm{SD})$ & $114.3(21.4)$ \\
\hline Median (minimum to maximum) & 116.1 (62.0 to 154.3$)$ \\
\hline Pre-treatment height SDS, mean (SD) & $-3.8(1.3)$ \\
\hline Median (minimum to maximum) & $-3.4(-9.4$ to -1.3$)$ \\
\hline Pre-treatment height velocity (cm/year), mean (SD) & $4.7(1.7)$ \\
\hline Median (minimum to maximum) & $4.7(0.5,10.6)$ \\
\hline Enrolment weight $(\mathrm{kg})$, mean $(\mathrm{SD})$ & $22.0(9.8)$ \\
\hline Median (min, max) & $21.0(5.0,59.6)$ \\
\hline
\end{tabular}


Characteristic

\begin{tabular}{|c|c|}
\hline Characteristic & EU-IGFD Registry $\left(\mathrm{N}=281^{\mathrm{a}}\right)$ \\
\hline Enrolment weight SDS, mean (SD) & $-3.3(1.4)$ \\
\hline Median (minimum to maximum) & $-3.1(-9.5$ to 0.1$)$ \\
\hline Enrolment BMI $\left(\mathrm{kg} / \mathrm{m}^{2}\right)$, mean (SD) & $16.1(2.9)$ \\
\hline Median (minimum to maximum) & 15.5 (11.2 to 31.5$)$ \\
\hline Enrolment BMI SDS, mean (SD) & $-0.7(1.4)$ \\
\hline Median (minimum to maximum) & $-0.8(-4.7$ to 4.0$)$ \\
\hline Bone age (years), mean (SD) & $8.6(3.5)$ \\
\hline Median (minimum to maximum) & $8.5(1.5,14.7)$ \\
\hline \multicolumn{2}{|c|}{ Maximum GH level (ng/mL), mean (SD) } \\
\hline Naive prepubertal patients & $26.6(24.6)$ \\
\hline Pubertal or previously treated patients & $28.9(49.7)$ \\
\hline \multicolumn{2}{|c|}{ Maximum GH level $(\mathrm{ng} / \mathrm{mL}$ ), median (minimum to maximum) } \\
\hline Naive prepubertal patients & $16.8(1.2,150.0)$ \\
\hline Pubertal or previously treated patients & $15.6(0.1,364.0)$ \\
\hline
\end{tabular}

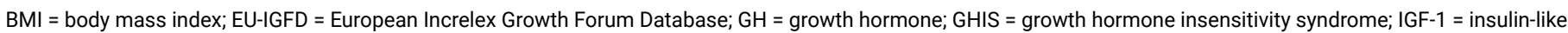
growth factor-1; SD = standard deviation; SDS = SDS = standard deviation score.

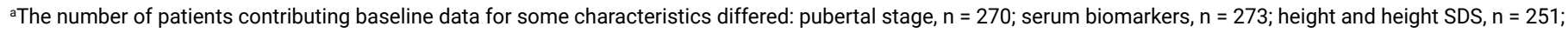
height velocity, $n=151$; weight and weight SDS, $n=254$; BMI and BMI SDS, $n=231$; bone age, $n=51$.

Source: Clinical Study Report for EU-IGFD Registry. ${ }^{20}$

considered related to the treatment. Safety information was collected at 2 and 5 years after the end of mecasermin therapy and included any significant diseases and disorders. Current illnesses considered to be related to mecasermin included malignancies; pre-malignancies; metabolism and nutrition disorders (hyperglycemia, pre-diabetes, and diabetes); cardiovascular disorders (high blood pressure, stroke, and left ventricular hypertrophy); ear-nose-throat disorders; neurologic disorders; bone disorders; immune system disorders; any disease or disorder requiring surgery; any other disease or disorder considered by the investigator to be related to mecasermin.

An AE was defined as any untoward medical occurrence in a patient administered mecasermin that did not necessarily have to have a causal relationship with the treatment. An SAE was defined as an AE occurring at any dose if it resulted in death, was life-threatening, resulted in hospitalization or prolonged existing hospitalization (excluding admission for social or administrative reasons), resulted in persistent or significant disability or incapacity, resulted in congenital anomaly or a birth defect in the offspring of a patient, or was an important medical event that could not have resulted in death, be life-threatening, or require hospitalization.

All notable harms were reported, including hypoglycemia, lipohypertrophy at injection sites, tonsillar hypertrophy, otitis media, hearing loss, sleep apnea, intracranial hypertension, papilledema, headache, acromegalic facial changes, edema, myalgia, gynecomastia, cardiomegaly, and other injection site reactions. 


\section{Statistical Analysis}

Analyses were presented for the whole registry population (including all patients who consented to participate who had at least 1 follow-up visit and had received at least 1 dose of mecasermin), the safety population (all patients who received at least 1 dose of mecasermin and with at least 1 follow-up visit or post-study safety data), and the long-term safety population (patients exposed to mecasermin for at least 3 years and who ended therapy, with at least 1 visit over 2 years after ending treatment). Subgroup data were presented for growth outcomes for treatment-naive or prepubertal patients, pubertal or previously treated patients, and patients with and without Laron syndrome. There was no formal sample-size calculation. The goal was to enrol as many patients as possible, or at minimum $60 \%$ of patients treated with mecasermin in Europe (enrolment target of 330 patients). This sample size would allow for a $95 \%$ probability of observing side effects, with a true incidence of 1 in 100 . For AEs with a known background rate, a doubling of the rate in the observed population could be detected with at least $80 \%$ power if the background rate is 3 in 100 .

Multiple regression analyses were performed based on follow-up visit data when at least 50 treatment-naive or prepubertal patients were treated for at least 1 year to identify predictive factors of change from baseline height SDS and height velocity in the first 4 years of treatment, final adult height, and change from baseline bone age. Covariates included in the models were sex, parental heights, Laron syndrome, height SDS at baseline, weight SDS at baseline, IGF-1 level at baseline, mean dose of mecasermin during the first year, age, and concomitant treatment with $\mathrm{GH}$.

All AEs and SAEs were summarized in tables based on the safety population. A safety analysis was performed every 6 months on uncleaned data. Once annually, a data-monitoring committee reviewed safety and efficacy data and made conclusions on the benefits and risks of mecasermin. Safety and efficacy analyses were performed every 2 years on cleaned data. AEs were considered in the post-treatment period (and in the long term safety population) if their onset was on or after the seventh day following the last mecasermin injection. An AE starting before the first mecasermin injection was considered a prior AE. At the time of the first event of hypoglycemia, the incidence by groups of age was presented. Predictive factors for hypoglycemia were analyzed using logistic regression, with age, sex, pubertal stage at baseline, mecasermin dose, weight, BMI, history of hypoglycemia, previous GH therapy, diagnosis of Laron syndrome, and baseline IGFBP-3 level as covariates.

Because this was a non-interventional study, visits were reflective of the usual interaction between patients and their physician. The number and frequency of follow-up and posttreatment visits were determined by investigator judgment, based on clinical need and product labelling recommendations. Time intervals were therefore constructed such that collected observations could be allocated to a particular time point for descriptive analyses. During follow-up, data were described every 3 months until 6 months of follow-up, and then every 6 months. If several visits occurred during the same window, the data collected at the nearest visit to the time point were used. For growth outcomes, the data were considered on treatment until 28 days after the treatment stop date.

Missing data were not replaced. When the date of medical history or disease diagnosis was missing or incomplete, it was assumed to have occurred before any study treatment. If a partial date and the associated information did not allow for a conclusion about the assignation to a group or category, all the possible groups or categories were considered. 
Where this was possible, the derivations based on a partial date were presented as superior inequalities.

\section{Patient Disposition}

A total of 319 patients were screened for enrolment in the EU-IGFD Registry from 118 study sites (Table 16). Data for 38 patients from non-participating sites were deleted, leaving 281 who had taken mecasermin at least once enrolled and treated. Of the enrolled patients, 275 $(98 \%)$ completed at least 1 follow-up visit and were included in the efficacy analyses. The safety analysis was based on 277 enrolled patients (99\%), with 17 (6\%) with at least a 2-year visit contributing to the long-term safety population. In total, 76 patients $(27 \%)$ completed the study (i.e., they stopped treatment and were followed until they achieved near-adult height [last height velocity $<1 \mathrm{~cm}$ per year] or adult height) and 164 (58\%) ended therapy definitively. The study lost 160 patients (57\%) to discontinuation. The most common reasons for discontinuation (frequency $>10 \%)$ included the attainment of adult height $(n=68 ; 43 \%)$ and losses to follow-up $(n=46 ; 29 \%)$. Few patients discontinued due to lack of effectiveness (i.e., inadequate growth, $n=5 ; 3 \%)$ or AEs $(n=4 ; 3 \%)$.

\section{Table 16: Patient Disposition for the EU-IGFD Registry}

\begin{tabular}{|l|c|}
\hline Patient disposition & EU-IGFD Registry \\
\hline Screened, $\mathrm{n}^{\mathrm{a}}$ & 319 \\
\hline Enrolled, $\mathrm{n}$ & 281 \\
\hline Treated patients, $\mathrm{n}(\%)^{\mathrm{b}}$ & $281(100)$ \\
\hline Registry patients, $\mathrm{n}(\%)^{\mathrm{c}}$ & $275(98)$ \\
\hline Safety population, $\mathrm{n}(\%)$ & $277(99)$ \\
\hline Long-term safety population, $\mathrm{n}(\%)$ & $17(6)$ \\
\hline Completed study, $\mathrm{n}(\%)^{\mathrm{d}}$ & $76(27)$ \\
\hline Ended therapy definitively, $\mathrm{n}(\%)$ & $164(58)$ \\
\hline Discontinued study, $\mathrm{n}(\%)$ & $160(57)$ \\
\hline Withdrawal of consent & $11(7)$ \\
\hline Attained adult height & $68(43)$ \\
\hline End of post-treatment follow-up & $5(3)$ \\
\hline Lost to follow-up & $46(29)$ \\
\hline Changed physician & $8(5)$ \\
\hline Lack of effectiveness & $5(3)$ \\
\hline Adverse event & $4(3)$ \\
\hline Other & $13(8)$ \\
\hline
\end{tabular}

EU-IGFD = European Increlex Growth Forum Database.

ancludes 281 enrolled patients and 38 not-enrolled patients who were deleted due to regulatory purposes.

bPatients who took mecasermin at least once.

'Patients who took mecasermin at least once and completed at least 1 follow-up visit.

dPatients who stopped the treatment and followed-up until "near-adult height" (last height velocity $<1 \mathrm{~cm}$ per year) $(n=10)$ or "attained adult height" ( $n=53)$ were reached. Source: Clinical Study Report for EU-IGFD Registry. ${ }^{20}$ 


\section{Exposure to Study Treatments}

At baseline, the median dosage of mecasermin was $40 \mathrm{mcg} / \mathrm{kg}$ (range $=10$ to 270) SC twice daily. At 6 months, the median dosage was $100 \mathrm{mcg} / \mathrm{kg}$ (range $=0$ to 270) SC twice daily. The median dosage between month 12 and month 120 was between $110 \mathrm{mcg} / \mathrm{kg} \mathrm{SC}$ twice daily and $120 \mathrm{mcg} / \mathrm{kg} \mathrm{SC}$ twice daily (the maximum recommended dosage in the product monograph). ${ }^{12} \mathrm{~A}$ total of 164 patients (58\%) ended therapy: 40 patients within the first year; 30 patients between 1 and 2 years; 29 patients between 2 and 3 years; 29 patients between 3 and 4 years; 13 patients between 4 and 5 years; 8 patients between 5 and 6 years; 7 patients between 6 and 7 years; 5 patients between 7 and 8 years; and 3 patients between 8 and 9 years of treatment. In patients eligible for the long-term safety follow-up analysis, the median treatment duration was 4.5 years (range $=3$ to 9 ), with $39 \%$ of patients being treated for 5 years or more. For the long-term safety population (17 patients with at least a 2-year visit), the median treatment duration was 4.0 years (range $=3$ to 6 ), with $25 \%$ being treated for 5 years or more.

Sixty-two patients (22\%) interrupted treatment for at least 1 month and $69(25 \%)$ experienced a modification of the treatment due to a shortage of mecasermin. A mecasermin shortage resulted in a dosage decrease, dose interruption, or dose decrease and interruption for 3 (1\%), $67(24 \%)$, and $1(<1 \%)$ patients, respectively. The mean duration of dose interruptions was 124 days $(S D=118)$. One patient experienced 2 dose interruptions.

Overall, 151 patients (54\%) were taking at least 1 chronic concomitant medication. The most common concomitant therapies (frequency $>5 \%$ ) were $\mathrm{GH}(n=41 ; 15 \%)$, thyroid hormone $(n=25 ; 9 \%)$, antibiotics $(n=22 ; 8 \%)$, bronchodilators $(n=19 ; 7 \%)$, gonadotropin-releasing hormone $(n=18 ; 6 \%)$, and other chronic medication (unspecified) $(n=71 ; 25 \%)$.

\section{Efficacy Outcomes}

Growth outcomes were evaluated during the treatment period for the whole registry population $(n=275)$, as well as for prepubertal patients naive to mecasermin $(n=162)$, and for pubertal or previously treated patients $(n=109)$. For some growth outcomes, data were also presented for patients concomitantly treated with $\mathrm{GH}(n=41)$ and for patients with $(n=$ 43) and without $(n=232)$ Laron syndrome. We have presented the efficacy data for the first 5 years of treatment.

\section{Height, Height Standard Deviation Score, and Height Velocity}

In the whole registry population, mean height at baseline was $114.3 \mathrm{~cm}(\mathrm{SD}=21.4)$. The mean change from baseline after 1 year of treatment was $+6.9 \mathrm{~cm}(\mathrm{SD}=2.4)$ (Table 17). The mean changes in height from baseline in years 2 through 5 of treatment were $+13.3 \mathrm{~cm}$ $(S D=3.6),+18.9 \mathrm{~cm}(S D=4.7),+24.2 \mathrm{~cm}(S D=4.7)$, and $+30.0 \mathrm{~cm}(S D=5.3)$, respectively. In prepubertal naive-to-mecasermin patients, the mean height at baseline was $107.2 \mathrm{~cm}(\mathrm{SD}=$ 20.4). The mean change from baseline after 1 year of treatment was $+7.2 \mathrm{~cm}(S D=2.2)$. The mean changes in height from baseline in years 2 through 5 of treatment were $+13.8 \mathrm{~cm}(\mathrm{SD}=$ $3.2),+19.5 \mathrm{~cm}(\mathrm{SD}=4.0),+25.0 \mathrm{~cm}(\mathrm{SD}=4.2)$, and $+30.9 \mathrm{~cm}(\mathrm{SD}=4.7)$, respectively. In pubertal or previously treated patients, the mean height at baseline was $124.1 \mathrm{~cm}(\mathrm{SD}=18.5)$. The mean change from baseline after 1 year of treatment was $6.4 \mathrm{~cm}(\mathrm{SD}=2.3)$. The mean changes in height from baseline in years 2 through 5 of treatment were $+12.5 \mathrm{~cm}(\mathrm{SD}=4.2)$, $+17.9 \mathrm{~cm}(\mathrm{SD}=6.0),+22.4 \mathrm{~cm}(\mathrm{SD}=5.4)$, and $+27.4 \mathrm{~cm}(\mathrm{SD}=6.1)$, respectively.

Among patients with Laron syndrome, mean height at baseline was $97.2 \mathrm{~cm}(S D=23.2)$. The mean change from baseline after 1 year of treatment was $+7.3 \mathrm{~cm}(\mathrm{SD}=3.1)$ (Table 18). The 
mean changes in height from baseline in years 2 through 5 of treatment were $+13.5 \mathrm{~cm}$ (SD = $5.1),+17.9 \mathrm{~cm}(\mathrm{SD}=7.2),+26.8(\mathrm{SD}=6.2)$, and $+29.2 \mathrm{~cm}(\mathrm{SD}=7.5)$, respectively.

In the whole registry population, mean height SDS at baseline was $-3.8(S D=1.3)$. The mean change from baseline after 1 year of treatment was $+0.4(S D=0.5)$ (Table 19). The mean changes in height SDS from baseline in years 2 through 5 of treatment were $+0.7(S D=0.7)$, $+0.8(S D=0.7),+1.0(S D=0.7)$, and + $1.1(S D=0.8)$, respectively. In prepubertal naiveto-mecasermin patients, the mean height SDS at baseline was $-3.8(S D=1.4)$. The mean change from baseline after 1 year of treatment was $+0.4(S D=0.4)$. There was evidence for an association between age at baseline and height SDS during the first year of treatment in

Table 17: Height During the First 5 Years of Treatment in the EU-IGFD Registry

\begin{tabular}{|c|c|c|c|}
\hline \multicolumn{4}{|c|}{ EU-IGFD Registry } \\
\hline Height (cm) & $\begin{array}{l}\text { Whole registry } \\
\qquad N=275\end{array}$ & $\begin{array}{l}\text { Prepubertal and } \\
\text { naive to mecasermin } \\
\qquad \mathrm{N}=162\end{array}$ & $\begin{array}{c}\text { Pubertal or } \\
\text { previously treated } \\
\qquad \mathrm{N}=109\end{array}$ \\
\hline Patients measured at baseline, $n$ & 249 & 147 & 98 \\
\hline Height, mean (SD) & $114.3(21.4)$ & $107.2(20.4)$ & $124.1(18.5)$ \\
\hline Patients measured at 1 year, $\mathrm{n}$ & 225 & 133 & 90 \\
\hline Height, mean (SD) & $120.6(21.7)$ & $113.8(21.2)$ & $129.7(18.6)$ \\
\hline Change from baseline, $\mathrm{n}$ & 204 & 120 & 82 \\
\hline Change from baseline $(\mathrm{cm})$, mean $(\mathrm{SD})$ & $6.9(2.4)$ & $7.2(2.2)$ & $6.4(2.3)$ \\
\hline Patients measured at 2 years, $n$ & 176 & 111 & 65 \\
\hline Height, mean (SD) & $124.9(21.7)$ & $119.5(21.1)$ & $134.2(19.7)$ \\
\hline Change from baseline, $n$ & 161 & 103 & 58 \\
\hline Change from baseline, mean (SD) & $13.3(3.6)$ & $13.8(3.2)$ & $12.5(4.2)$ \\
\hline Patients measured at 3 years, $n$ & 131 & 87 & 43 \\
\hline Height, mean (SD) & $129.6(21.5)$ & $126.1(21.7)$ & $135.9(19.4)$ \\
\hline Change from baseline, $\mathrm{n}$ & 119 & 81 & 37 \\
\hline Change from baseline, mean (SD) & $18.9(4.7)$ & $19.5(4.0)$ & $17.9(6.0)$ \\
\hline Patients measured at 4 years, $\mathrm{n}$ & 97 & 65 & 32 \\
\hline Height, mean (SD) & $130.8(19.7)$ & $127.2(19.4)$ & $138.1(18.3)$ \\
\hline Change from baseline, $\mathrm{n}$ & 87 & 60 & 27 \\
\hline Change from baseline, mean (SD) & $24.2(4.7)$ & $25.0(4.2)$ & $22.4(5.4)$ \\
\hline Patients measured at 5 years, $n$ & 70 & 53 & 17 \\
\hline Height, mean (SD) & $132.4(18.5)$ & $131.6(18.7)$ & $134.9(18.1)$ \\
\hline Change from baseline, $\mathrm{n}$ & 62 & 46 & 16 \\
\hline Change from baseline, mean (SD) & $30.0(5.3)$ & $30.9(4.7)$ & $27.4(6.1)$ \\
\hline
\end{tabular}

EU-IGFD = European Increlex Growth Forum Database; SD = standard deviation. Source: Clinical Study Report for EU-IGFD Registry. ${ }^{20}$ 
multivariate linear regression $(-0.02$; estimated $95 \% \mathrm{Cl},-0.04$ to $-0.00 ; \mathrm{P}=0.024)$. The mean changes in height SDS in years 2 through 5 of treatment were $+0.7(S D=0.6),+0.9(S D=$ $0.6),+1.1(S D=0.6)$, and + 1.2 (SD = 0.8), respectively. There was evidence for an association between height SDS at baseline and height SDS during the second year of treatment in multivariate linear regression $(-0.13$; estimated $95 \% \mathrm{Cl},-0.21$ to $-0.05 ; \mathrm{P}=0.001)$. In pubertal or previously treated patients, the mean height SDS at baseline was $-3.7(S D=1.3)$. The mean change from baseline after 1 year of treatment was $+0.2(S D=0.5)$. The mean changes in height SDS from baseline in years 2 through 5 of treatment were $+0.5(S D=0.7),+0.6(S D=$ $0.9),+0.7(S D=0.7)$, and $+0.6(0.7)$, respectively. In patients concomitantly treated with $\mathrm{GH}$, the mean height SDS at baseline was $-3.7(S D=1.4)$. The mean change from baseline after

\section{Table 18: Height During the First 5 Years of Treatment in the EU-IGFD Registry Among Patients With Laron Syndrome}

\begin{tabular}{|c|c|}
\hline \multicolumn{2}{|c|}{ EU-IGFD Registry } \\
\hline Height (cm) & $\begin{array}{l}\text { Patients with Laron syndrome } \\
\qquad \mathrm{N}=43\end{array}$ \\
\hline Patients measured at baseline, $n$ & 16 \\
\hline Height, mean (SD) & $97.2(23.2)$ \\
\hline Patients measured at 1 year, $n$ & 14 \\
\hline Height, mean (SD) & $106.9(20.6)$ \\
\hline Change from baseline, $\mathrm{n}$ & 13 \\
\hline Change from baseline, mean (SD) & $7.3(3.1)$ \\
\hline Patients measured at 2 years, $n$ & 16 \\
\hline Height, mean (SD) & $110.9(20.5)$ \\
\hline Change from baseline, $n$ & 14 \\
\hline Change from baseline, mean (SD) & $13.5(5.1)$ \\
\hline Patients measured at 3 years, $n$ & 13 \\
\hline Height, mean (SD) & $114.7(20.0)$ \\
\hline Change from baseline, $n$ & 11 \\
\hline Change from baseline, mean (SD) & $17.9(7.2)$ \\
\hline Patients measured at 4 years, $n$ & 9 \\
\hline Height, mean (SD) & $118.9(22.2)$ \\
\hline Change from baseline, $n$ & 7 \\
\hline Change from baseline, mean (SD) & $26.8(6.2)$ \\
\hline Patients measured at 5 years, $n$ & 7 \\
\hline Height, mean (SD) & $114.4(17.5)$ \\
\hline Change from baseline, $n$ & 7 \\
\hline Change from baseline, mean (SD) & $29.2(7.5)$ \\
\hline
\end{tabular}

EU-IGFD = European Increlex Growth Forum Database; SD = standard deviation.

Source: Clinical Study Report for EU-IGFD Registry. ${ }^{20}$ 
1 year of treatment was $+0.2(S D=0.5)$. The mean changes in height SDS from baseline in years 2 through 5 of treatment were $+0.4(S D=0.7),+0.6(S D=0.7),+0.8(S D=0.8)$, and $+0.7(S D=1.0)$, respectively.

Among patients with Laron syndrome, mean height SDS at baseline was $-5.0(S D=1.8)$. The mean change from baseline after 1 year of treatment was $+0.5(S D=0.6)($ Table 20). The mean changes in height SDS from baseline in years 2 through 5 of treatment were +0.9 $(S D=0.8),+0.8(S D=0.9),+1.1(S D=1.0)$, and $+1.1(S D=1.1)$, respectively. Among patients without Laron syndrome, the mean height SDS at baseline was $-3.6(S D=1.1)$. The mean change from baseline after 1 year of treatment was $+0.3(S D=0.4)$. The mean changes in height SDS from baseline in years 2 through 5 of treatment were $+0.6(S D=0.6),+0.8(S D=$ $0.7),+1.0(S D=0.6)$, and $+1.1(S D=0.7)$, respectively.

In the whole registry population, mean height velocity at baseline was $4.7 \mathrm{~cm}$ per year (SD = 1.7). The mean change from baseline after 1 year of treatment was $+2.0 \mathrm{~cm}$ per year (SD = 2.6) (Table 21). The mean changes in height velocity from baseline in years 2 through 5 of treatment were $+1.4 \mathrm{~cm}$ per year $(S D=2.4),+0.7 \mathrm{~cm}$ per year $(S D=2.8),+0.5 \mathrm{~cm}$ per year $(S D=2.2)$, and $+0.2 \mathrm{~cm}$ per year $(S D=1.9)$, respectively. In prepubertal naive-to-mecasermin patients, the mean height velocity at baseline was $4.8 \mathrm{~cm}$ per year $(S D=1.8)$. The mean change from baseline after 1 year of treatment was $+2.5 \mathrm{~cm}$ per year $(S D=2.5)$. The mean changes in height velocity in years 2 through 5 of treatment were $+1.8 \mathrm{~cm}$ per year $(\mathrm{SD}=$ $2.3),+1.1 \mathrm{~cm}$ per year $(2.6),+0.8 \mathrm{~cm}$ per year $(\mathrm{SD}=2.2)$, and $+0.8 \mathrm{~cm}$ per year $(\mathrm{SD}=1.8)$, respectively. There was evidence for an association between Laron syndrome and height velocity during the second year of treatment in multivariate linear regression (1.13; estimated $95 \% \mathrm{Cl}, 0.00$ to $2.27 ; \mathrm{P}=0.050$ ). In pubertal or previously treated patients, the mean height velocity at baseline was $4.6 \mathrm{~cm}$ per year $(S D=1.7)$. The mean change from baseline after 1 year of treatment was $+1.5 \mathrm{~cm}$ per year $(S D=2.6)$. The mean changes in height velocity from baseline in years 2 through 5 of treatment were $+0.7 \mathrm{~cm}$ per year $(S D=2.4),-0.1 \mathrm{~cm}$ per year $(S D=3.1),-0.1 \mathrm{~cm}$ per year $(S D=2.3)$, and $-0.8 \mathrm{~cm}$ per year $(S D=1.6)$, respectively. In patients concomitantly treated with $\mathrm{GH}$, mean height velocity at baseline was $4.6 \mathrm{~cm}$ per year $(S D=1.8)$. The mean changes from baseline after 1 and 2 years of treatment were $+1.2 \mathrm{~cm}$ per year $(S D=3.0)$ and $+0.7 \mathrm{~cm}$ per year $(S D=2.9)$, respectively.

For patients with Laron syndrome, mean height velocity at baseline was $4.8 \mathrm{~cm}$ per year $(S D=1.3)$. The mean change from baseline after 1 year of treatment was $+1.3 \mathrm{~cm}$ per year $(S D=2.5)$ (Table 22). The mean changes in height velocity from baseline in years 2 through 4 of treatment were $+0.7 \mathrm{~cm}$ per year $(S D=2.5),-1.9 \mathrm{~cm}$ per year $(S D=3.0)$, and $-0.3 \mathrm{~cm}$ per year $(S D=2.7)$, respectively. In patients without Laron syndrome, mean height velocity at baseline was $4.7 \mathrm{~cm}$ per year $(\mathrm{SD}=1.8)$. The mean change from baseline after 1 year of treatment was $+2.2 \mathrm{~cm}$ per year $(\mathrm{SD}=2.6)$. The mean changes in height $\mathrm{SDS}$ from baseline in years 2 through 5 of treatment were $+1.5(S D=2.3),+1.3(S D=2.5),+0.7(S D=2.2)$, and $+0.2(S D=1.8)$, respectively.

\section{Predicted Adult Height and Final Adult Height}

In the whole registry population, the mean final adult height and final adult height score were $152.0 \mathrm{~cm}(S D=13.6)$ and $-3.0(S D=1.7)$, respectively (Table 23). The mean difference between the predicted adult height and final adult height was $-5.8 \mathrm{~cm}(S D=11.7)$. There was an association between height SDS at baseline in multivariate linear regression (7.29; estimated 95\% Cl, 5.31 to 9.28; $\mathrm{P}<0.001)$ and final adult height. There was evidence for an association between height SDS at baseline in multivariate linear regression (1.02; estimated 
$95 \% \mathrm{Cl}, 0.81$ to $1.23 ; \mathrm{P}<0.001)$ and final adult height SDS. Among prepubertal naive-tomecasermin patients, the mean final adult height and final adult height SDS were $158.6 \mathrm{~cm}$ $(S D=12.6)$ and $-2.3(S D=1.2)$, respectively. The mean difference between the predicted adult height and final adult height was $-1.8 \mathrm{~cm}(S D=10.8)$. There was evidence for an association between age at baseline in multivariate linear regression (1.24; estimated $95 \% \mathrm{Cl}, 0.70$ to 1.78 ; $\mathrm{P}<0.001)$, a predicted adult height in multivariate linear regression $(0.33$; estimated $95 \% \mathrm{Cl}$, 0.18 to $0.47 ; P<0.001$ ), and a height SDS at baseline in multivariate linear regression (3.39; estimated $95 \% \mathrm{Cl}, 1.33$ to $5.44 ; \mathrm{P}=0.003$ ) and final adult height. There was evidence for an association between predicted adult height in multivariate linear regression (0.05; estimated

Table 19: Height SDS During the First 5 Years of Treatment in the EU-IGFD Registry

\begin{tabular}{|c|c|c|c|c|}
\hline \multicolumn{5}{|c|}{ EU-IGFD Registry } \\
\hline Height standard deviation score & $\begin{array}{l}\text { Whole registry } \\
\qquad N=275\end{array}$ & $\begin{array}{l}\text { Prepubertal and } \\
\text { naive to mecasermin } \\
\qquad \mathrm{N}=162\end{array}$ & $\begin{array}{l}\text { Pubertal or previously } \\
\text { treated with } \\
\text { mecasermin } \\
\mathrm{N}=109\end{array}$ & $\begin{array}{l}\text { Concomitantly } \\
\text { treated with } \mathrm{GH} \\
\qquad \mathrm{N}=41\end{array}$ \\
\hline Patients measured at baseline, $\mathrm{n}$ & 249 & 147 & 98 & 38 \\
\hline Height SDS, mean (SD) & $-3.8(1.3)$ & $-3.8(1.4)$ & $-3.7(1.3)$ & $-3.7(1.4)$ \\
\hline Patients measured at 1 year, $\mathrm{n}$ & 225 & 133 & 90 & 30 \\
\hline Height SDS, mean (SD) & $-3.5(1.4)$ & $-3.4(1.4)$ & $-3.5(1.4)$ & $-3.4(1.5)$ \\
\hline Change from baseline, $\mathrm{n}$ & 204 & 120 & 82 & 28 \\
\hline Change from baseline, mean (SD) & $0.4(0.5)$ & $0.4(0.4)$ & $0.2(0.5)$ & $0.2(0.5)$ \\
\hline Patients measured at 2 years, $n$ & 176 & 111 & 65 & 22 \\
\hline Height SDS, mean (SD) & $-3.3(1.5)$ & $-3.2(1.4)$ & $3.4(1.6)$ & $-3.1(1.8)$ \\
\hline Change from baseline, $n$ & 161 & 103 & 58 & 20 \\
\hline Change from baseline, mean (SD) & $0.7(0.7)$ & $0.7(0.6)$ & $0.5(0.7)$ & $0.4(0.7)$ \\
\hline Patients measured at 3 years, $n$ & 131 & 87 & 43 & 12 \\
\hline Height SDS, mean (SD) & $-3.0(1.6)$ & $-2.9(1.5)$ & $-3.4(1.8)$ & $-3.1(1.6)$ \\
\hline Change from baseline, $\mathrm{n}$ & 119 & 81 & 37 & 11 \\
\hline Change from baseline, mean (SD) & $0.8(0.7)$ & $0.9(0.6)$ & $0.6(0.9)$ & $0.6(0.7)$ \\
\hline Patients measured at 4 years, $n$ & 97 & 65 & 32 & 11 \\
\hline Height SDS, mean (SD) & $-3.0(1.67)$ & $-2.7(1.5)$ & $-3.6(1.8)$ & $-3.2(2.1)$ \\
\hline Change from baseline, $n$ & 87 & 60 & 27 & 11 \\
\hline Change from baseline, mean (SD) & $1.0(0.7)$ & $1.1(0.6)$ & $0.7(0.7)$ & $0.8(0.8)$ \\
\hline Patients measured at 5 years, $n$ & 70 & 53 & 17 & 6 \\
\hline Height SD score, mean (SD) & $-2.8(1.8)$ & $-2.5(1.6)$ & $-3.7(2.1)$ & $-3.5(2.9)$ \\
\hline Change from baseline, $\mathrm{n}$ & 62 & 46 & 16 & 6 \\
\hline Change from baseline, mean (SD) & $1.1(0.8)$ & $1.2(0.8)$ & $0.6(0.7)$ & $0.7(1.0)$ \\
\hline
\end{tabular}

EU-IGFD = European Increlex Growth Forum Database; GH = growth hormone; SD = standard deviation; SDS = standard deviation score. Source: Clinical Study Report for EU-IGFD Registry. ${ }^{20}$ 
$95 \% \mathrm{Cl}, 0.03$ to $0.07 ; \mathrm{P}<0.001)$ and height SDS at baseline in multivariate linear regression $(0.43$; estimated $95 \% \mathrm{Cl}, 0.09$ to $0.78 ; \mathrm{P}=0.016)$ and final adult height SDS.

Health-Related Quality of Life

Measurements of HRQoL were taken for 47 patients from France and/or their parents; however, baseline and follow-up data were only available for 2 patients and could not be summarized in this report.

Table 20: Height SDS During the First 5 Years of Treatment in the EU-IGFD Registry Among Patients With and Without Laron Syndrome

\begin{tabular}{|c|c|c|}
\hline \multicolumn{3}{|c|}{ EU-IGFD Registry } \\
\hline Height standard deviation score & $\begin{array}{l}\text { Patients with Laron syndrome } \\
\qquad \mathrm{N}=43\end{array}$ & $\begin{array}{l}\text { Patients without Laron syndrome } \\
\qquad \mathrm{N}=232\end{array}$ \\
\hline Patients measured at baseline, $\mathrm{n}$ & 35 & 214 \\
\hline Height SDS, mean (SD) & $-5.0(1.8)$ & $-3.6(1.1)$ \\
\hline Patients measured at 1 year, $\mathrm{n}$ & 36 & 189 \\
\hline Height SDS, mean (SD) & $-4.4(1.7)$ & $-3.3(1.2)$ \\
\hline Change from baseline, $n$ & 30 & 174 \\
\hline Change from baseline, mean (SD) & $0.5(0.6)$ & $0.3(0.4)$ \\
\hline Patients measured at 2 years, $n$ & 31 & 145 \\
\hline Height SDS, mean (SD) & $-4.4(1.6)$ & $-3.0(1.3)$ \\
\hline Change from baseline, $\mathrm{n}$ & 26 & 135 \\
\hline Change from baseline, mean (SD) & $0.9(0.8)$ & $0.6(0.6)$ \\
\hline Patients measured at 3 years, $n$ & 23 & 108 \\
\hline Height SDS, mean (SD) & $-4.2(1.9)$ & $-2.8(1.4)$ \\
\hline Change from baseline, $\mathrm{n}$ & 20 & 99 \\
\hline Change from baseline, mean (SD) & $0.8(0.9)$ & $0.8(0.7)$ \\
\hline Patients measured at 4 years, $\mathrm{n}$ & 21 & 76 \\
\hline Height SDS, mean (SD) & $-4.0(2.0)$ & $-2.7(1.5)$ \\
\hline Change from baseline, $\mathrm{n}$ & 18 & 69 \\
\hline Change from baseline, mean (SD) & $1.1(1.0)$ & $1.0(0.6)$ \\
\hline Patients measured at 5 years, $n$ & 17 & 53 \\
\hline Height SDS, mean (SD) & $-4.2(2.0)$ & $-2.4(1.5)$ \\
\hline Change from baseline, $\mathrm{n}$ & 14 & 48 \\
\hline Change from baseline, mean (SD) & $1.1(1.1)$ & $1.1(0.7)$ \\
\hline
\end{tabular}

EU-IGFD = European Increlex Growth Forum Database; SD = standard deviation; SDS = standard deviation score. Source: Clinical Study Report for EU-IGFD Registry. ${ }^{20}$ 


\section{Bone Age}

In the whole registry population, bone age at baseline was 8.6 years $(S D=3.5)$. The mean change from baseline after 1 year of treatment was +1.2 years $(S D=0.9)($ Table 24). The mean changes in bone age from baseline in years 2 and 3 of treatment were +2.1 years $(S D=$ $0.9)$ and +3.4 years $(S D=0.6)$, respectively. In prepubertal naive-to-mecasermin patients, the mean bone age at baseline was 7.3 years $(S D=3.1)$. The mean change from baseline after 1 year of treatment was +1.1 years $(S D=0.5)$. The mean changes in bone age in years 2 and 3 of treatment were +2.3 years $(S D=0.6)$ and +3.3 years $(S D=0.6)$, respectively. In pubertal

\section{Table 21: Height Velocity During the First 5 Years of Treatment in the EU-IGFD Registry}

\begin{tabular}{|c|c|c|c|c|}
\hline \multicolumn{5}{|c|}{ EU-IGFD Registry } \\
\hline Height velocity (cm per year) & $\begin{array}{l}\text { Whole registry } \\
\qquad N=275\end{array}$ & $\begin{array}{l}\text { Prepubertal and } \\
\text { naive to mecasermin } \\
\qquad N=162\end{array}$ & $\begin{array}{l}\begin{array}{l}\text { Pubertal or } \\
\text { previously treated } \\
N=109\end{array}\end{array}$ & $\begin{array}{c}\text { Concomitantly } \\
\text { treated with GH } \\
\qquad N=41\end{array}$ \\
\hline Patients measured at baseline, $n$ & 151 & 80 & 70 & 27 \\
\hline Height velocity, mean (SD) & $4.7(1.7)$ & $4.8(1.8)$ & $4.6(1.7)$ & $4.6(1.8)$ \\
\hline Patients measured at 1 year, $\mathrm{n}$ & 197 & 118 & 77 & 27 \\
\hline Height velocity, mean (SD) & $6.9(2.3)$ & $7.2(2.1)$ & $6.3(2.4)$ & $5.9(2.1)$ \\
\hline Change from baseline, $\mathrm{n}$ & 123 & 67 & 55 & 19 \\
\hline Change from baseline, mean (SD) & $2.0(2.6)$ & $2.5(2.5)$ & $1.5(2.6)$ & $1.2(3.0)$ \\
\hline Patients measured at 2 years, $n$ & 138 & 89 & 49 & 19 \\
\hline Height velocity, mean (SD) & $6.1(1.9)$ & $6.2(1.6)$ & $5.9(2.4)$ & $5.3(2.2)$ \\
\hline Change from baseline, $n$ & 82 & 49 & 33 & 13 \\
\hline Change from baseline, mean (SD) & $1.4(2.4)$ & $1.8(2.3)$ & $0.7(2.4)$ & $0.7(2.9)$ \\
\hline Patients measured at 3 years, $n$ & 110 & 76 & 34 & NA \\
\hline Height velocity, mean (SD) & $5.6(2.1)$ & $5.9(1.8)$ & $5.1(2.7)$ & NA \\
\hline Change from baseline, $n$ & 61 & 41 & 20 & NA \\
\hline Change from baseline, mean (SD) & $0.7(2.8)$ & $1.1(2.6)$ & $-0.1(3.1)$ & NA \\
\hline Patients measured at 4 years, $\mathrm{n}$ & 74 & 51 & 23 & NA \\
\hline Height velocity, mean (SD) & $5.3(1.8)$ & $5.6(1.6)$ & $4.8(2.1)$ & NA \\
\hline Change from baseline, $n$ & 36 & 24 & 12 & NA \\
\hline Change from baseline, mean (SD) & $0.5(2.2)$ & $0.8(2.2)$ & $-0.1(2.3)$ & NA \\
\hline Patients measured at 5 years, $n$ & 47 & 34 & 13 & NA \\
\hline Height velocity, mean (SD) & $5.0(1.8)$ & $5.2(1.7)$ & $4.5(1.8)$ & NA \\
\hline Change from baseline, $\mathrm{n}$ & 23 & 15 & 8 & NA \\
\hline Change from baseline, mean (SD) & $0.2(1.9)$ & $0.8(1.8)$ & $-0.8(1.6)$ & NA \\
\hline
\end{tabular}

EU-IGFD = European Increlex Growth Forum Database; GH = growth hormone; NA = not available; SD = standard deviation. Source: Clinical Study Report for EU-IGFD Registry. ${ }^{20}$ 
or previously treated patients, the mean bone age at baseline was 10.3 years $(S D=3.3)$ years.

The mean change from baseline after 1 year of treatment was + 1.4 years $(S D=1.3)$.

In the whole registry population, the difference between bone age and chronological age at baseline was -2.0 years $(S D=1.2)$. The mean change from baseline after 1 year of treatment was +0.1 years $(S D=0.9)($ Table 25). The mean changes in the difference between bone age and chronological age in years 2 and 3 of treatment were +0.1 years $(S D=0.9)$ and +0.3 years $(S D=0.6)$, respectively. In prepubertal naive-to-mecasermin patients, the difference between bone age and chronological age at baseline was -1.9 years $(S D=1.0)$. The mean

Table 22: Height Velocity During the First 5 Years of Treatment in the EU-IGFD Registry Among Patients With and Without Laron Syndrome

\begin{tabular}{|c|c|c|}
\hline \multicolumn{3}{|c|}{ EU-IGFD Registry } \\
\hline & $\begin{array}{l}\text { Patients with Laron } \\
\text { syndrome }\end{array}$ & $\begin{array}{l}\text { Patients without Laron } \\
\text { syndrome }\end{array}$ \\
\hline Height velocity (cm per year) & $N=43$ & \\
\hline Patients measured at baseline, $n$ & 21 & 130 \\
\hline Height velocity, mean (SD) & $4.8(1.3)$ & $4.7(1.8)$ \\
\hline Patients measured at 1 year, $\mathrm{n}$ & 29 & 168 \\
\hline Height velocity, mean (SD) & $7.0(2.8)$ & $6.9(2.2)$ \\
\hline Change from baseline, $\mathrm{n}$ & 18 & 105 \\
\hline Change from baseline, mean (SD) & $1.3(2.5)$ & $2.2(2.6)$ \\
\hline Patients measured at 2 years, $n$ & 21 & 117 \\
\hline Height velocity, mean (SD) & $6.0(2.3)$ & $6.1(1.9)$ \\
\hline Change from baseline, $\mathrm{n}$ & 13 & 69 \\
\hline Change from baseline, mean (SD) & $0.7(2.5)$ & $1.5(2.3)$ \\
\hline Patients measured at 3 years, $n$ & 21 & 89 \\
\hline Height velocity, mean (SD) & $4.5(2.6)$ & $5.9(1.9)$ \\
\hline Change from baseline, $\mathrm{n}$ & 10 & 51 \\
\hline Change from baseline, mean (SD) & $-1.9(3.0)$ & $1.3(2.5)$ \\
\hline Patients measured at 4 years, $\mathrm{n}$ & 16 & 58 \\
\hline Height velocity, mean (SD) & $5.1(1.8)$ & $5.4(1.8)$ \\
\hline Change from baseline, $\mathrm{n}$ & 6 & 30 \\
\hline Change from baseline, mean (SD) & $-0.3(2.7)$ & $0.7(2.2)$ \\
\hline Patients measured at 5 years, $n$ & NA & 37 \\
\hline Height velocity, mean (SD) & NA & $4.9(1.8)$ \\
\hline Change from baseline, $\mathrm{n}$ & NA & 20 \\
\hline Change from baseline, mean (SD) & NA & $0.2(1.8)$ \\
\hline
\end{tabular}

EU-IGFD = European Increlex Growth Forum Database; NA = not available; SD = standard deviation. Source: Clinical Study Report for EU-IGFD Registry. ${ }^{20}$ 
change from baseline after 1 year of treatment was +0.1 years $(S D=0.6)$. The mean changes in the difference between bone age and chronological age in years 2 and 3 of treatment were +0.2 years $(S D=0.6)$ and +0.2 years $(S D=0.6)$, respectively. In pubertal or previously treated patients, the difference between bone age and chronological age at baseline was $-2.0(\mathrm{SD}=$ 1.4). The mean change from baseline after 1 year of treatment was +0.3 years $(S D=1.3)$.

\section{Weight and Weight Standard Deviation Score}

In the whole registry population, mean weight at baseline was $22.0 \mathrm{~kg}(\mathrm{SD}=9.8)$ and changed by a mean of $+3.7 \mathrm{~kg}(S D=2.2)$ during the first year of treatment (Table 26). The mean changes in weight from baseline in years 2 through 5 of treatment were $+7.4 \mathrm{~kg}$ $(S D=4.0),+10.4 \mathrm{~kg}(\mathrm{SD}=5.1),+12.9 \mathrm{~kg}(\mathrm{SD}=5.9)$, and $+16.2 \mathrm{~kg}(\mathrm{SD}=6.5)$, respectively. In prepubertal naive-to-mecasermin patients, the mean weight at baseline was $18.4 \mathrm{~kg}(\mathrm{SD}=$ $7.3)$ and changed by a mean of $+3.3 \mathrm{~kg}(\mathrm{SD}=1.8)$ during the first year of treatment. The mean changes in weight in years 2 through 5 of treatment were $+6.6 \mathrm{~kg}(\mathrm{SD}=3.6),+9.9 \mathrm{~kg}$ $(S D=5.0),+12.0 \mathrm{~kg}(S D=5.4)$, and $+15.6 \mathrm{~kg}(S D=6.1)$, respectively. In pubertal or previously treated patients, the mean weight at baseline was $27.0 \mathrm{~kg}(\mathrm{SD}=10.4)$ and changed by a mean $+4.2 \mathrm{~kg}(\mathrm{SD}=2.6)$ during the first year of treatment. The mean changes in weight in years 2 through 5 of treatment were $+8.6 \mathrm{~kg}(\mathrm{SD}=4.4) \mathrm{kg},+11.7 \mathrm{~kg}(\mathrm{SD}=5.2),+15.0 \mathrm{~kg}(\mathrm{SD}=6.5)$, and $+18.1 \mathrm{~kg}(\mathrm{SD}=7.4)$, respectively.

In the whole registry population, the mean weight SDS at baseline was $-3.3(S D=1.4)$ and changed by a mean of $+0.4(S D=0.6)$ during the first year of treatment (Table 27). The mean

\section{Table 23: Predicted Adult Height and Final Adult Height in the EU-IGFD Registry Among Patients Who Achieved Adult Height}

\begin{tabular}{|c|c|c|}
\hline \multicolumn{3}{|c|}{ EU-IGFD Registry } \\
\hline Predicted $^{\mathrm{a}}$ and final adult height & $\begin{array}{l}\text { Whole registry } \\
\qquad \begin{array}{l}\mathrm{N}=76\end{array}\end{array}$ & $\begin{array}{c}\text { Prepubertal and } \\
\text { naive to mecasermin } \\
\qquad \mathrm{N}=76\end{array}$ \\
\hline Predicted adult height $(\mathrm{cm}), \mathrm{n}$ & 52 & 24 \\
\hline Mean (SD) & $158.9(14.4)$ & $158.6(12.6)$ \\
\hline Median (minimum to maximum) & 163.0 (124 to 182$)$ & 162.0 (131 to 182$)$ \\
\hline Final adult height $(\mathrm{cm}), \mathrm{n}$ & 75 & 32 \\
\hline Mean (SD) & $152.0(13.6)$ & $155.1(13.2)$ \\
\hline Median (minimum to maximum) & 154.0 (104 to 180$)$ & 156.2 (104 to 180$)$ \\
\hline Difference (final - predicted adult height) $(\mathrm{cm})$, & 52 & 24 \\
\hline Mean (SD) & $-5.8(11.7)$ & $-1.8(10.8)$ \\
\hline Median (minimum to maximum) & $-4.6(-31$ to 34$)$ & $-2.7(-18$ to 34$)$ \\
\hline Final adult height SDS, $\mathrm{n}$ & 75 & 32 \\
\hline Mean (SD) & $-3.0(1.7)$ & $-2.3(1.2)$ \\
\hline Median (minimum to maximum) & $-2.5(-8$ to 0$)$ & $-2.1(-7$ to 0$)$ \\
\hline
\end{tabular}

EU-IGFD = European Increlex Growth Forum Database; SD = standard deviation.

aThe method used to calculate predicted adult height was not reported.

Source: Clinical Study Report for EU-IGFD Registry. ${ }^{20}$ 
changes in weight SDS from baseline in years 2 through 5 of treatment were $+0.8(S D=0.9)$, $+1.0(\mathrm{SD}=1.0),+1.2(\mathrm{SD}=1.2)$, and + $1.6(\mathrm{SD}=1.2)$, respectively. In prepubertal naive-tomecasermin patients, the mean weight SDS at baseline was $-3.4(S D=1.4)$ and changed by a mean of $+0.5(S D=0.7)$ during the first year of treatment. The mean changes in weight SDS in years 2 through 5 of treatment were + $0.8(S D=1.0),+1.0(S D=1.1),+1.3(S D=1.3)$, and $+1.6(S D=1.4)$, respectively. In pubertal or previously treated patients, the mean weight SDS at baseline was $-3.1(S D=1.5)$ and changed by a mean of $+0.4(S D=0.6)$ during the first year of treatment. The mean changes in weight SDS in years 2 through 5 of treatment were $+0.7(S D=0.8),+0.9(S D=0.9),+1.2(S D=0.8)$, and $+1.4(S D=0.5)$, respectively.

\section{BMI Standard Deviation Score}

In the whole registry population, mean BMI SDS at baseline was $-0.7(S D=1.4)$. The mean change from baseline after 1 year of treatment was $+0.2(S D=0.7)$ (Table 28). The mean changes in BMI SDS from baseline in years 2 through 5 of treatment were $+0.3(S D=0.7)$, $+0.4(S D=0.8),+0.4(S D=0.9)$, and + $0.6(S D=1.0)$, respectively. In prepubertal naive-tomecasermin patients, the mean BMI SDS at baseline was $-0.8(S D=1.3)$. The mean change from baseline after 1 year of treatment was $+0.2(S D=0.7)$. The mean changes in BMI SDS in years 2 through 5 of treatment were $+0.3(S D=0.7),+0.4(S D=0.8),+0.4(S D=1.0)$, and $+0.6(S D=1.1)$, respectively. In pubertal or previously treated patients, the mean weight SDS at baseline was $-0.7(S D=1.5)$. The mean change from baseline after 1 year of treatment

\section{Table 24: Bone Age During the First 5 Years of Treatment in the EU-IGFD Registry}

\begin{tabular}{|l|c|c|c|}
\hline & EU-IGFD Registry & Phole registry \\
Bone age (years) & $\begin{array}{c}\text { Prepubertal and naive } \\
\text { to mecasermin } \\
\mathrm{N}=162\end{array}$ & $\begin{array}{c}\text { Pubertal or previously } \\
\text { treated } \\
\mathrm{N}=109\end{array}$ \\
\hline Patients measured at baseline, $\mathrm{n}$ & 49 & 29 & 19 \\
\hline Bone age, mean (SD) & $8.6(3.5)$ & $7.3(3.1)$ & $10.3(3.3)$ \\
\hline Patients measured at 1 year, $\mathrm{n}$ & 74 & 44 & 30 \\
\hline Bone age, mean (SD) & $8.7(3.8)$ & $7.3(3.3)$ & $10.7(3.7)$ \\
\hline Change from baseline, $n$ & 17 & 10 & 7 \\
\hline Change from baseline, mean (SD) & $1.2(0.9)$ & $1.1(0.5)$ & NA \\
\hline Patients measured at 2 years, $n$ & 54 & 40 & NA \\
\hline Bone age, mean (SD) & $9.6(3.8)$ & $8.6(3.4)$ & NA \\
\hline Change from baseline, $n$ & 12 & 11 & NA \\
\hline Change from baseline, mean (SD) & $2.1(0.9)$ & $2.3(0.6)$ & NA \\
\hline Patients measured at 3 years, $n$ & 38 & 24 & NA \\
\hline Bone age, mean (SD) & $10.9(3.6)$ & $9.8(3.3)$ & NA \\
\hline Change from baseline, $n$ & 9 & 8 & NA \\
\hline Change from baseline, mean (SD) & $3.4(0.6)$ & $3.3(0.6)$ & \\
\hline
\end{tabular}

EU-IGFD = European Increlex Growth Forum Database; NA = not available; SD = standard deviation. Source: Clinical Study Report for EU-IGFD Registry. ${ }^{20}$ 
was $+0.2(S D=0.7)$. The mean changes in weight SDS in years 2 through 5 of treatment were $+0.3(S D=0.7),+0.4(S D=0.6),+0.5(S D=0.8)$, and +0.6 (SD = 0.8), respectively.

\section{Harms}

Only those harms identified in the review protocol are reported. Harms were reported for the treatment period $(n=277)$ and for the post-treatment period $(n=164)$. Harms were also reported for the long-term safety population $(n=17)$. See Table 29 for detailed data for harms during the treatment period.

\section{Adverse Events}

A total of 185 patients (67\%) had at least 1 AE during treatment. The most common AEs (frequency $>10 \%$ ) were metabolism and nutrition disorders ( $n=73 ; 26 \%$ ); investigations $(n=61 ; 22 \%)$; skin and subcutaneous tissue disorders $(n=48 ; 17 \%)$; respiratory, thoracic, and mediastinal disorders $(n=39 ; 14 \%)$; nervous system disorders $(n=38 ; 14 \%)$; infections and infestations $(n=34 ; 12 \%)$; and general disorders and administration site conditions $(n=32 ; 12 \%)$.

\section{Table 25: Difference Between Bone Age and Chronological Age During the First 3 Years of} Treatment in the EU-IGFD Registry

\begin{tabular}{|c|c|c|c|}
\hline \multicolumn{4}{|c|}{ EU-IGFD Registry } \\
\hline $\begin{array}{l}\text { Difference between bone age and chronological } \\
\text { age (years) }\end{array}$ & $\begin{array}{l}\text { Whole registry } \\
\qquad \mathrm{N}=275\end{array}$ & $\begin{array}{l}\text { Prepubertal and naive } \\
\text { to mecasermin } \\
\qquad \mathrm{N}=162\end{array}$ & $\begin{array}{l}\text { Pubertal or previously } \\
\text { treated } \\
\mathrm{N}=109\end{array}$ \\
\hline Patients measured at baseline, $n$ & 49 & 29 & 19 \\
\hline $\begin{array}{l}\text { Difference between bone age and chronological } \\
\text { age, mean (SD) }\end{array}$ & $-2.0(1.2)$ & $-1.9(1.0)$ & $-2.0(1.4)$ \\
\hline Patients measured at 1 year, $\mathrm{n}$ & 74 & 44 & 30 \\
\hline $\begin{array}{l}\text { Difference between bone age and chronological } \\
\text { age, mean (SD) }\end{array}$ & $-1.9(1.4)$ & $-1.8(1.2)$ & $-2.0(1.7)$ \\
\hline Change from baseline, $\mathrm{n}$ & 17 & 10 & 7 \\
\hline Change from baseline, mean (SD) & $0.1(0.9)$ & $0.1(0.6)$ & $0.3(1.3)$ \\
\hline Patients measured at 2 years, $n$ & 54 & 40 & NA \\
\hline $\begin{array}{l}\text { Difference between bone age and chronological } \\
\text { age, mean (SD) }\end{array}$ & $-1.8(1.4)$ & $-1.7(1.4)$ & NA \\
\hline Change from baseline, $n$ & 12 & 11 & NA \\
\hline Change from baseline, mean (SD) & $0.1(0.9)$ & $0.2(0.6)$ & NA \\
\hline Patients measured at 3 years, $n$ & 38 & 24 & NA \\
\hline $\begin{array}{l}\text { Difference between bone age and chronological } \\
\text { age, mean (SD) }\end{array}$ & $-1.7(1.2)$ & $-1.7(1.1)$ & NA \\
\hline Change from baseline, $n$ & 9 & 8 & NA \\
\hline Change from baseline, mean (SD) & $0.3(0.6)$ & $0.2(0.6)$ & NA \\
\hline
\end{tabular}

EU-IGFD = European Increlex Growth Forum Database; NA = not available; SD = standard deviation; SDS = standard deviation score. Source: Clinical Study Report for EU-IGFD Registry. ${ }^{20}$ 
During the post-treatment period, 39 AEs were reported in 21 patients (13\%). During the longterm safety period, 5 patients (29\%) experienced 6 AEs (tonsilitis, cyclic vomiting syndrome, hearing loss, tonsillar hypertrophy, decreased thyroxine free, and decreased vitamin D).

\section{Serious Adverse Events}

Fifty-nine patients (21\%) had at least 1 SAE during treatment. The most common SAEs (occurring in at least $5 \%$ of patients) were infections and infestations ( $n=13 ; 5 \%)$; respiratory, thoracic, and mediastinal disorders $(n=12 ; 4 \%)$; injury, poisoning, and procedural complications $(n=10 ; 4 \%)$; metabolism and nutrition disorders $(n=9 ; 3 \%)$; gastrointestinal

Table 26: Body Weight During the First 5 Years of Treatment in the EU-IGFD Registry

\begin{tabular}{|c|c|c|c|}
\hline \multicolumn{4}{|c|}{ EU-IGFD Registry } \\
\hline Weight (kg) & $\begin{array}{l}\text { Whole registry } \\
\qquad \mathrm{N}=275\end{array}$ & $\begin{array}{l}\text { Prepubertal and naive } \\
\text { to mecasermin } \\
\mathrm{N}=162\end{array}$ & $\begin{array}{l}\text { Pubertal or previously } \\
\text { treated } \\
N=109\end{array}$ \\
\hline Patients measured at baseline, $n$ & 252 & 149 & 99 \\
\hline Weight, mean (SD) & $22.0(9.8)$ & $18.4(7.3)$ & $27.0(10.4)$ \\
\hline Patients measured at 1 year, $\mathrm{n}$ & 225 & 133 & 90 \\
\hline Weight, mean (SD) & $25.7(11.1)$ & $21.6(8.8)$ & $31.3(11.3)$ \\
\hline Change from baseline, $\mathrm{n}$ & 206 & 121 & 83 \\
\hline Change from baseline, mean (SD) & $3.7(2.2)$ & $3.3(1.8)$ & $4.2(2.6)$ \\
\hline Patients measured at 2 years, $n$ & 176 & 111 & 65 \\
\hline Weight, mean (SD) & $28.7(12.4)$ & $24.7(10.1)$ & $35.7(12.9)$ \\
\hline Change from baseline, $\mathrm{n}$ & 161 & 103 & 58 \\
\hline Change from baseline, mean (SD) & $7.4(4.0)$ & $6.6(3.6)$ & $8.6(4.4)$ \\
\hline Patients measured at 3 years, $n$ & 131 & 87 & 43 \\
\hline Weight, mean (SD) & $31.1(13.0)$ & $28.1(11.6)$ & 36.9 \\
\hline Change from baseline, $\mathrm{n}$ & 119 & 81 & 37 \\
\hline Change from baseline, mean (SD) & $10.4(5.1)$ & $9.9(5.0)$ & $11.7(5.2)$ \\
\hline Patients measured at 4 years, $n$ & 97 & 65 & 32 \\
\hline Weight, mean (SD) & $32.3(13.0)$ & $28.9(11.4)$ & $39.0(13.6)$ \\
\hline Change from baseline, $\mathrm{n}$ & 87 & 60 & 27 \\
\hline Change from baseline, mean (SD) & $12.9(5.9)$ & $12.0(5.4)$ & $15.0(6.5)$ \\
\hline Patients measured at 5 years, $n$ & 70 & 53 & 17 \\
\hline Weight, mean (SD) & $33.3(12.2)$ & $31.7(11.6)$ & $38.1(13.3)$ \\
\hline Change from baseline, $\mathrm{n}$ & 63 & 47 & 16 \\
\hline Change from baseline, mean (SD) & $16.2(6.5)$ & $15.6(6.1)$ & $18.1(7.4)$ \\
\hline
\end{tabular}

EU-IGFD = European Increlex Growth Forum Database; SD = standard deviation. Source: Clinical Study Report for EU-IGFD Registry. ${ }^{20}$ 
disorders ( $n=7 ; 3 \%)$; nervous system disorders $(n=6 ; 2 \%)$; and musculoskeletal and connective tissue disorders $(n=5 ; 2 \%)$.

During the post-treatment period, 17 SAEs were reported. During the long-term safety period, 2 SAEs (tonsillitis and cyclic vomiting syndrome) were reported.

Withdrawals Due to Adverse Events

Fifteen (5\%) patients withdrew from the study due to AEs during treatment.

Table 27: Body Weight SDS During the First 5 Years of Treatment in the EU-IGFD Registry

\begin{tabular}{|c|c|c|c|}
\hline \multicolumn{4}{|c|}{ EU-IGFD Registry } \\
\hline Weight standard deviation score & $\begin{array}{l}\text { Whole registry } \\
\qquad \mathrm{N}=275\end{array}$ & $\begin{array}{l}\text { Prepubertal and naive } \\
\text { to mecasermin } \\
\mathrm{N}=162\end{array}$ & $\begin{array}{c}\text { Pubertal or previously } \\
\text { treated } \\
N=109\end{array}$ \\
\hline Patients measured at baseline, $n$ & 252 & 149 & 99 \\
\hline Weight SDS, mean (SD) & $-3.3(1.4)$ & $-3.4(1.4)$ & $-3.1(1.5)$ \\
\hline Patients measured at 1 year, $\mathrm{n}$ & 225 & 133 & 90 \\
\hline Weight SDS, mean (SD) & $-2.8(1.6)$ & $-2.9(1.6)$ & $-2.7(1.4)$ \\
\hline Change from baseline, $\mathrm{n}$ & 206 & 121 & 83 \\
\hline Change from baseline, mean (SD) & $0.4(0.6)$ & $0.5(0.7)$ & $0.4(0.6)$ \\
\hline Patients measured at 2 years, $n$ & 176 & 111 & 65 \\
\hline Weight SDS, mean (SD) & $-2.5(1.7)$ & $2.6(1.8)$ & $-2.3(1.5)$ \\
\hline Change from baseline, $n$ & 161 & 103 & 58 \\
\hline Change from baseline, mean (SD) & $0.8(0.9)$ & $0.8(1.0)$ & $0.7(0.8)$ \\
\hline Patients measured at 3 years, $n$ & 131 & 87 & 43 \\
\hline Weight SDS, mean (SD) & $-2.3(1.9)$ & $-2.3(2.1)$ & $-2.3(1.6)$ \\
\hline Change from baseline, $\mathrm{n}$ & 119 & 81 & 37 \\
\hline Change from baseline, mean (SD) & $1.0(1.0)$ & $1.0(1.1)$ & $0.9(0.9)$ \\
\hline Patients measured at 4 years, $\mathrm{n}$ & 97 & 65 & 32 \\
\hline Weight SDS, mean (SD) & $-2.1(2.0)$ & $-2.0(2.2)$ & $-2.3(1.5)$ \\
\hline Change from baseline, $\mathrm{n}$ & 87 & 60 & 27 \\
\hline Change from baseline, mean (SD) & $1.2(1.2)$ & $1.3(1.3)$ & $1.2(0.8)$ \\
\hline Patients measured at 5 years, $n$ & 70 & 53 & 17 \\
\hline Weight SDS, mean (SD) & $-1.8(1.9)$ & $-1.7(2.0)$ & $-2.1(1.6)$ \\
\hline Change from baseline, $\mathrm{n}$ & 63 & 47 & 16 \\
\hline Change from baseline, mean (SD) & $1.6(1.2)$ & $1.6(1.4)$ & $1.4(0.5)$ \\
\hline
\end{tabular}

EU-IGFD = European Increlex Growth Forum Database; SD = standard deviation; SDS = standard deviation score. Source: Clinical Study Report for EU-IGFD Registry. ${ }^{20}$ 


\section{Mortality}

Two (1\%) patients died during treatment (1 patient from myelodysplastic syndrome and 1 due to complications of a bone marrow transplant). No patient died during the post-treatment or long-term safety periods.

\section{Notable Harms}

The most frequently reported notable harms included hypoglycemia $(n=68 ; 25 \%)$, lipohypertrophy at the injection site $(n=33 ; 12 \%)$, and tonsillar hypertrophy $(n=25 ; 9 \%)$.

\section{Table 28: BMI SDS During the First 5 Years of Treatment in the EU-IGFD Registry}

\begin{tabular}{|c|c|c|c|}
\hline \multicolumn{4}{|c|}{ EU-IGFD Registry } \\
\hline BMI standard deviation score & $\begin{array}{c}\text { Whole registry } \\
\qquad N=275\end{array}$ & $\begin{array}{l}\text { Prepubertal and } \\
\text { naive to mecasermin } \\
\qquad \mathrm{N}=162\end{array}$ & $\begin{array}{l}\text { Pubertal or previously } \\
\text { treated } \\
\mathrm{N}=109\end{array}$ \\
\hline Patients measured at baseline, $\mathrm{n}$ & 229 & 133 & 92 \\
\hline BMI SDS, mean (SD) & $-0.7(1.4)$ & $-0.8(1.3)$ & $-0.7(1.5)$ \\
\hline Patients measured at 1 year, $\mathrm{n}$ & 225 & 133 & 90 \\
\hline BMI SDS, mean (SD) & $-0.5(1.4)$ & $-0.5(1.4)$ & $-0.3(1.4)$ \\
\hline Change from baseline, $\mathrm{n}$ & 184 & 106 & 76 \\
\hline Change from baseline, mean (SD) & $0.2(0.7)$ & $0.2(0.7)$ & $0.2(0.7)$ \\
\hline Patients measured at 2 years, $n$ & 176 & 111 & 65 \\
\hline BMI SDS, mean (SD) & $-0.3(1.5)$ & $-0.4(1.5)$ & $-0.0(1.4)$ \\
\hline Change from baseline, $n$ & 146 & 92 & 54 \\
\hline Change from baseline, mean (SD) & $0.3(0.7)$ & $0.3(0.7)$ & $0.3(0.7)$ \\
\hline Patients measured at 3 years, $n$ & 131 & 87 & 43 \\
\hline BMI SDS, mean (SD) & $-0.3(1.5)$ & $-0.4(1.4)$ & $-0.1(1.7)$ \\
\hline Change from baseline, $n$ & 109 & 74 & 34 \\
\hline Change from baseline, mean (SD) & $0.4(0.8)$ & $0.4(0.8)$ & $0.4(0.6)$ \\
\hline Patients measured at 4 years, $\mathrm{n}$ & 97 & 65 & 32 \\
\hline BMI SDS, mean (SD) & $-0.1(1.5)$ & $-0.2(1.6)$ & $-0.0(1.3)$ \\
\hline Change from baseline, $\mathrm{n}$ & 80 & 56 & 24 \\
\hline Change from baseline, mean (SD) & $0.4(0.9)$ & $0.4(1.0)$ & $0.5(0.8)$ \\
\hline Patients measured at 5 years, $n$ & 70 & 53 & 17 \\
\hline BMI SDS, mean (SD) & $0.0(1.5)$ & $-0.1(1.6)$ & $0.4(1.3)$ \\
\hline Change from baseline, $\mathrm{n}$ & 58 & 42 & 16 \\
\hline Change from baseline, mean (SD) & $0.6(1.0)$ & $0.6(1.1)$ & $0.6(0.8)$ \\
\hline
\end{tabular}

$\mathrm{BMI}=$ body mass index; EU-IGFD = European Increlex Growth Forum Database; SD = standard deviation; SDS = standard deviation score. Source: Clinical Study Report for EU-IGFD Registry. ${ }^{20}$ 
Myalgia ( $n=4 ; 1 \%$ ), benign, malignant, and unspecified neoplasms (including cysts and polyps) $(n=2 ; 1 \%)$, and intracranial hypertension $(n=1 ; 0.4 \%)$, were reported less frequently.

Seven patients (4\%) reported notable harms during the post-treatment period. These included 2 events of tonsillar hypertrophy. One patient (6\%) reported tonsillar hypertrophy during the long-term safety period.

\section{Critical Appraisal} Internal Validity

The EU-IGFD Registry provides real-world evidence for evaluating growth and safety outcomes among patients treated with mecasermin for SPIGFD. By design, patients and their treating physicians were aware of the prescribed treatment (i.e., the study was open-label), and the only available comparator was within-patient change from baseline. Because this was an observational study and there was no control group for the analysis of efficacy or harms, the findings are at high risk of confounding. The investigators did not control for known prognostic factors or effect modifiers (e.g., environmental or socioeconomic factors) within the analyses, and establishing a causal link between the treatment and the growth outcomes is not possible. The lack of control group precludes making strong conclusions regarding the benefits and harms of the treatment. As the analyses were not adjusted for multiple comparisons, the risk of type I error is increased.

It is unclear from the Clinical Study Report whether patients were enrolled consecutively, creating a potential for selection bias (i.e., enrolment of patients who would most likely benefit from the treatment) that could overestimate the treatment effect. Patients who were currently participating in a clinical trial of mecasermin were excluded and it is unclear how these patients may have differed from those included in the EU-IGFD Registry. It is unclear whether the study was powered to detect statistically significant changes in growth outcomes (although these were not tested statistically), as sample size and power calculations were not reported and the size of the whole registry population fell short of the enrolment target $(n=330)$. The sample size was substantially smaller for longer-term follow-up (e.g., $n=47$ for height velocity after 5 years of treatment and $n=75$ for final adult height). With respect to the analysis of harms, the sample size was not large enough to observe side effects with a true incidence of up to 1 in 100 . Only 17 patients were eligible for the long-term safety analysis.

SDSs were calculated in the EU-IGFD Registry using growth curves designed for the general population, and need to be interpreted in context as the results could have differed depending on the reference standard used. For France and southern European countries, height and weight SDS were calculated using age- and sex-dependent Sempé reference means and SDs. ${ }^{46}$ Height percentiles on these charts differ substantially from WHO child growth standards used in Canada (e.g., at all ages, $25 \%$ of French children fall below the 10 th percentile for height based on WHO standards), ${ }^{49}$ exemplifying the complexity of interpreting SDSs calculated using different standards. The BMI SDSs were calculated using the French National Plan for Nutrition and Health reference means and SD values. ${ }^{47} \mathrm{~A}$ comparison of BMI standards showed that the French standards classified fewer children as overweight and obese compared to WHO standards (to varying degrees, by age and sex)..$^{50}$ For the UK, Belgium, Sweden, and Poland, height, weight, and BMI SDSs were calculated using UK reference values. Of the available reference values in use in the $\mathrm{UK}^{48}$ it is unclear which ones were applied to the EU-IGFD Registry. Given differences in the reference populations and methods used to develop various growth standards, the currency and overall validity of the 
Table 29: Summary of Harms During Treatment for the EU-IGFD Registry

\begin{tabular}{|c|c|}
\hline \multicolumn{2}{|c|}{ EU-IGFD Registry N = 277} \\
\hline \multicolumn{2}{|c|}{ Patients with $\geq 1$ adverse event } \\
\hline $\mathrm{n}(\%)$ & $185(67)$ \\
\hline \multicolumn{2}{|l|}{ Most common events, ${ }^{\text {a }} \mathrm{n}(\%)$} \\
\hline Metabolism and nutrition disorders & $73(26)$ \\
\hline Investigations & $61(22)$ \\
\hline Skin and subcutaneous tissue disorders & $48(17)$ \\
\hline Respiratory, thoracic, and mediastinal disorders & $39(14)$ \\
\hline Nervous system disorders & $38(14)$ \\
\hline Infections and infestations & $34(12)$ \\
\hline General disorders and administration site conditions & $32(12)$ \\
\hline \multicolumn{2}{|c|}{ Patients with $\geq 1$ serious adverse event } \\
\hline $\mathrm{n}(\%)$ & $59(21)$ \\
\hline \multicolumn{2}{|l|}{ Most common events, ${ }^{\mathrm{b}} \mathrm{n}(\%)$} \\
\hline Infections and infestations & $13(5)$ \\
\hline Respiratory, thoracic, and mediastinal disorders & $12(4)$ \\
\hline Injury, poisoning, and procedural complications & $10(4)$ \\
\hline Metabolism and nutrition disorders & $9(3)$ \\
\hline Gastrointestinal disorders & $7(3)$ \\
\hline Nervous system disorders & $6(2)$ \\
\hline Musculoskeletal and connective tissue disorders & $5(2)$ \\
\hline \multicolumn{2}{|c|}{ Patients who stopped treatment due to adverse events } \\
\hline $\mathrm{n}(\%)$ & $15(5)$ \\
\hline \multicolumn{2}{|c|}{ Deaths } \\
\hline $\mathrm{n}(\%)$ & $2(1)$ \\
\hline \multicolumn{2}{|c|}{ Notable harms } \\
\hline Hypoglycemia, n (\%) & $68(25)$ \\
\hline Lipohypertrophy at the injection site, n (\%) & $33(12)$ \\
\hline Tonsillar hypertrophy, n (\%) & $25(9)$ \\
\hline Myalgia, n (\%) & $4(1)$ \\
\hline $\begin{array}{l}\text { Benign, malignant, and unspecified neoplasms (including cysts } \\
\text { and polyps), } \mathrm{n}(\%)\end{array}$ & $2(1)$ \\
\hline Intracranial hypertension, n (\%) & $1(0.4)$ \\
\hline
\end{tabular}

EU-IGFD = European Increlex Growth Forum Database.

${ }^{a}$ Frequency greater than $10 \%$. Common adverse events are summarized by the Medical Dictionary of Regulatory Activities system organ class for patients who received at 
SDSs calculated in this study are uncertain. It is also uncertain whether pooling data that were calculated using various growth standards (i.e., French and UK) is appropriate.

All patients, their parents or caregivers, the treating clinicians, and the outcome assessors were aware of the prescribed treatment. When patients and/or their caregivers are aware of the treatment that they are receiving, performance bias is possible (i.e., they may perceive or detect an enhanced treatment effect). Detection bias is also possible because the outcome assessors may exaggerate treatment effects if they are aware of the treatment and the purpose of the study. With respect to the growth outcomes in the EU-IGFD Registry, the risks of both performance and detection bias are low because all were objectively measured. It is possible that common subjective harms known to be associated with mecasermin could have been over-reported, as patients and their treating clinicians knew of the treatment received and of their participation in a registry study.

A large proportion of enrolled patients discontinued the study early for reasons other than completing the course of treatment or achieving final adult height $(n=76 ; 27 \%)$. Of these, 46 (16\%) were lost to follow-up, 8 (3\%) changed physicians, 5 (2\%) stopped treatment due to lack of efficacy, 4 (1\%) stopped treatment due to AEs, and 13 (5\%) withdrew for other, unspecified reasons. No data were collected after withdrawal. Additionally, baseline data were missing for a large proportion of enrolled patients across all outcomes (i.e., $n=32[11 \%]$ for height and height SDS; $n=130$ [46\%] for height velocity; $n=232$ [93\%] for bone age; $n=37$ [13\%] for weight; $n=52$ [19\%] for BMI SDS). Six enrolled patients (2\%) did not complete at least 1 follow-up visit and were excluded from the primary efficacy analyses. No imputations or other statistical methods were used to account for missing data and there is a high risk that the efficacy and harms data could be biased due to missing data (although the direction of the bias is unclear).

One-quarter ( $n=69$ ) of those enrolled were affected by a mecasermin shortage over the course of the study, resulting in a dosage decrease or dose interruption. These dosage decreases and interruption could have attenuated the growth outcomes and harms observed in the EU-IGFD Registry.

\section{External Validity}

The EU-IGFD Registry included an international group of patients treated with mecasermin for growth retardation. There were no inclusion criteria specific to Health Canada's approved indication for mecasermin (e.g., specifications for height, basal IGF-1 level, GH sufficiency, and exclusion of secondary forms of IGF-1 deficiency) ${ }^{12}$; however, the European Medicines Agency's therapeutic indication for mecasermin is identical to Health Canada's. ${ }^{12,23}$ In contrast with Study 1419 , in which $89 \%$ of patients were diagnosed with Laron syndrome, only $15 \%$ of patients in the EU-IGFD Registry had Laron syndrome, which is the most common known cause of SPIGFD. ${ }^{7}$ The cause of short stature for the remaining $85 \%$ of patients was not specified, and it is unclear whether nonhormonal causes were ruled out. The mean enrolment BMI SDS was -0.7 (SD = 1.4) with a wide range of -4.7 to 4.0 , and it is plausible that some patients may have been undernourished. Diagnostic criteria for beginning mecasermin therapy were not described, and likely varied across patients and treatment indications in various countries. The mean height SDS was substantially short, on average, at baseline, with a mean of $-3.8(S D=13)$. Because the severity of short stature was highly variable (baseline 
height SDSs ranged from -9.4 to -1.3 ), some patients did not meet the specifications for height SDS outlined in the product monograph. ${ }^{12}$ Similarly, height velocity ranged from $0.5 \mathrm{~cm}$ per year to $10.6 \mathrm{~cm}$ per year at baseline.

Sixty-three percent of patients in the EU-IGFD Registry were male and the median chronological age at baseline was 9.6 years (range $=0.4$ to 19.1 ), although bone age was delayed, at a median of 8.5 years (range $=1.5$ to 14.7). These patients would be eligible for treatment based on age, according to the product monograph, ${ }^{12}$ but are likely older than the optimal starting age of treatment. The results could have differed had patients started treatment sooner to optimize the treatment response. Although SPIGFD affects males and females equally, the imbalance in the proportion of males and females enrolled is unlikely to affect the generalizability of the findings as the treatment is expected to have an equivalent effect on males and females.

The exposure to mecasermin in the EU-IGFD Registry is likely reflective of typical exposures for patients who would be treated in the Canadian context. At baseline, the median dosage of mecasermin was $40 \mathrm{mcg} / \mathrm{kg} \mathrm{SC}$ twice daily, the recommended starting dosage in the product monograph. ${ }^{12}$ Some patients were receiving a smaller or substantially larger dosage (range = $10 \mathrm{mcg} / \mathrm{kg}$ to $270 \mathrm{mcg} / \mathrm{kg}$ SC twice daily); however, not all patients were naive to mecasermin at baseline. The median dosage between month 12 and month 120 of treatment was between $110 \mathrm{mcg} / \mathrm{kg}$ SC twice daily and $120 \mathrm{mcg} / \mathrm{kg}$ SC twice daily, the maximum recommended in the product monograph. ${ }^{12}$ Some patients received a higher dosage, up to a maximum of 270 $\mathrm{mcg} / \mathrm{kg}$ SC twice daily. Exposures outside of the recommended dosage are likely similar to what would occur in clinical practice, where dosages would be prescribed on a case-by-case basis based on treatment response and tolerability. Sixty-nine patients (25\% of those enrolled) were affected by a mecasermin shortage over the course of the study, resulting in a dosage decrease or dose interruption. The results may not be generalizable to contexts where such dosage decreases or interruptions do not occur. Concomitant medications taken by patients during the study are similar to those that would be expected in Canadian clinical practice.

\section{Polish Study on Increlex}

One report of 1 single-arm trial ${ }^{24}$ was included. The report presents data on the growth outcomes and harms observed in the first 3 years of treatment with mecasermin in patients with SPIGFD from Poland. In the absence of randomized trial data for the review question, the Polish Study on Increlex provides valuable real-world evidence for the use of mecasermin.

\section{Methods}

The Polish Study on Increlex was a multi-centre, single-arm trial that enrolled patients with SPIGFD to investigate the efficacy and harms of mecasermin treatment for patients with SPIGFD during the first 3 years in which it was covered by the therapeutic program in Poland.

\section{Populations}

\section{Inclusion and Exclusion Criteria}

Patients were eligible for inclusion if they were diagnosed with SPIGFD according to Polish criteria, i.e., they presented with extremely short stature below $-3.0 \mathrm{SD}$ for age and sex and they had an IGF-1 concentration below the 2.5th percentile for age and sex confirmed in nocturnal surge and/or stimulation tests. An IGF-1 generation test was carried out in all patients to assess the insensitivity to $\mathrm{GH}$. The test was carried out over 4 days, whereby the initial concentration of IGF-1 was assessed, then at 6 p.m. the GH was administered subcutaneously for 4 consecutive days in a daily dose of $0.033 \mathrm{mg} / \mathrm{kg}$. The IGF-1 
concentration was assessed in the morning, and an increase of IGF-1 concentration of less than $15 \mathrm{ng} / \mathrm{mL}$ indicated complete (severe) IGF-1 deficiency. A change of $15 \mathrm{ng} / \mathrm{mL}$ to 160 $\mathrm{ng} / \mathrm{mL}$ suggested partial IGF-1 deficiency, and a change of more than $160 \mathrm{ng} / \mathrm{mL}$ excluded primary IGF-1 deficiency.

\section{Baseline Characteristics}

This study included the first 27 children in Poland treated with mecasermin, including 22 males (81\%) and 5 females (19\%) (Table 30). The mean chronological age at baseline was 10.1 years (range $=2.8$ to 16.2 ). Nearly all patients $(n=25 ; 93 \%)$ were prepubescent. Two patients (7\%) were at Tanner stage 2 of sexual development. The mean pre-treatment height SDS and height velocity were -3.5 (range $=-6.5$ to -2.3$)$ and $4.6 \mathrm{~cm}$ per year (range $=0.9$ to 7.5), respectively. The mean pre-treatment weight SDS was -3.1 (range $=-5.8$ to -1.2 ). The mean BMI SDS at baseline was -1.8 (SD = 1.3). The mean enrolment IGF-1 SDS and maximum GH level were -2.2 (range $=-2.6$ to -1.6 ) and $24.4 \mathrm{ng} / \mathrm{mL}$ (range $=10.7$ to 50.1 ), respectively. Notably, 1 patient did not have a height lower than -3.0 SD for age and sex, and another did not have an initial IGF-1 below the 2.5 th percentile for age and sex. One had a pre-treatment height velocity of $7.5 \mathrm{~cm}$ per year; however, the patient still had short stature and a height SDS of -3.3 .

Table 30: Summary of Baseline Characteristics for the Polish Study on Increlex

\begin{tabular}{|l|c|}
\hline Characteristic & Polish Study on Increlex (N = 27) \\
\hline Pre-treatment age (years), mean & 2.8 to 16.2 \\
\hline Minimum to maximum & \\
\hline Sex, $\mathrm{n}(\%)$ & $22(81)$ \\
\hline Male & $5(19)$ \\
\hline Female & -2.2 \\
\hline Enrolment IGF-1 SDS, mean & -2.6 to -1.6 \\
\hline Minimum to maximum & -3.5 \\
\hline Pre-treatment height SDS, mean & $-6.5,-2.3$ \\
\hline Minimum to maximum & 4.6 \\
\hline Pre-treatment height velocity (cm/year), mean & $0.9,7.5$ \\
\hline Minimum to maximum & -3.1 \\
\hline Enrolment weight SDS, mean & $-5.8,-1.2$ \\
\hline Minimum to maximum & $-1.8(1.3)$ \\
\hline Enrolment BMI SDS, mean (SD) & $-2.6,-1.6$ \\
\hline Minimum to maximum & 24.4 \\
\hline Maximum GH level (ng/mL), mean & $10.7,50.1$ \\
\hline Minimum to maximum & \\
\hline
\end{tabular}

$\mathrm{BMI}=$ body mass index; $\mathrm{GH}$ = growth hormone; IGF-1 = insulin-like growth factor 1; SD = standard deviation; SDS = standard deviation score. Source: Petriczko et al. (2019). ${ }^{24}$ 


\section{Interventions}

All patients received mecasermin. There was no control group and the only comparator was within-patient change from baseline. The initial dosage of $40 \mathrm{mcg} / \mathrm{kg}$ SC twice daily was increased over time. The maximum dosage was $120 \mathrm{mcg} / \mathrm{kg}$ SC twice daily.

\section{Outcome Measures}

Growth outcomes were measured every 3 months up to 36 months (3 years) of treatment, and included height SDS, height velocity, weight SDS, and BMI SDS. Side effects (AES) were collected from all patients. Every 3 months, the concentration of IGF-1, IGFBP-3, and fasting glucose were measured. Every 6 months, the concentration of thyrotropin, free thyroxine, and serum electrolytes were measured. Oral glucose tolerance tests were carried out every 12 months, percentage of glycated hemoglobin was measured, cardiac consultation with echocardiography and laryngological consultation with audiometric examination was done, and bone age assessments were carried out. Neuroimaging was done annually.

\section{Statistical Analysis}

Changes from baseline mean values were investigated using the Mann-Whitney $U$ test.

\section{Patient Disposition}

Twenty-seven patients were enrolled in the study (Table 31). Five patients (19\%) discontinued the study early. Two patients (7\%) discontinued early due to AEs - 1 after 24 months and the second after 30 months of treatment. The other 3 patients (11\%) completed treatment during the study. The primary efficacy analysis included the 25 patients who completed the study.

\section{Exposure to Study Treatments}

The initial dosage of mecasermin was $40 \mathrm{mcg} / \mathrm{kg}$ SC twice daily. Within 12 months, the average dosage increased to $80 \mathrm{mcg} / \mathrm{kg} \mathrm{SC}$ twice daily (range $=60 \mathrm{mcg} / \mathrm{kg}$ to $120 \mathrm{mcg} / \mathrm{kg} \mathrm{SC}$ twice daily). The final average dosage was $100 \mathrm{mcg} / \mathrm{kg} \mathrm{SC}$ twice daily (range $=80 \mathrm{mcg} / \mathrm{kg}$ to $120 \mathrm{mcg} / \mathrm{kg}$ SC twice daily). Compliance with the daily injections was not measured.

\section{Efficacy}

Growth outcomes were evaluated after 3 years for all patients who completed the study $(\mathrm{n}=25)$.

\section{Table 31: Patient Disposition for the Polish Study on Increlex}

\begin{tabular}{|l|c|}
\hline Patient disposition & Polish study on Increlex \\
\hline Enrolled, $\mathrm{n}$ & $3(11)$ \\
\hline Completed treatment, $\mathrm{n}(\%)^{\mathrm{a}}$ & $5(19)$ \\
\hline Discontinued study, $\mathrm{n}(\%)$ & Reasons for discontinuation, $\mathrm{n}(\%)$ \\
\hline \multicolumn{2}{|c|}{$2(7)$} \\
\hline Adverse event & $3(11)$ \\
\hline Completed treatment & \\
\hline
\end{tabular}

Source: Petriczko et al. (2019). ${ }^{24}$ 
Height Standard Deviation Score and Height Velocity

The mean height SDS increased from $-3.5(S D=0.8)$ at baseline to $-2.3(S D=0.9)$ after 3 years of treatment $(P<0.01)$ (Table 32). The mean increase in height was 1.5 SDs $(S D=1.1)$. There was an apparent decrease in mean height SDS during the 21st month of the treatment, which may be due to missing results for some patients at that time point (according to the investigators). There was an apparent increase in mean height velocity from $4.5 \mathrm{~cm}$ per year $(S D=1.6)$ at baseline to $4.9 \mathrm{~cm}$ per year $(S D=1.8)$ after 3 years of treatment. The greatest apparent increase was during the first year of treatment, when the mean height velocity was $7.8 \mathrm{~cm}$ per year $(S D=1.9)$. In the second year of treatment, the mean height velocity was 6.3 $\mathrm{cm}$ per year $(\mathrm{SD}=0.8)$.

Health-Related Quality of Life

The Polish Study on Increlex did not measure HRQoL.

\section{Skeletal Maturation}

Skeletal maturation (e.g., bone age, bone age SDS) was measured in the Polish Study on Increlex, but the findings were not included in the published report.

\section{Weight Standard Deviation Score}

The mean weight SDS increased from $-3.1(S D=1.1)$ at baseline to $-2.0(S D=1.0)$ after 3 years of treatment $(P<0.01)$. The mean weight gain over 3 years was 1.2 SDs $(S D=0.8)$.

BMI Standard Deviation Score

The mean BMI SDS increased from $-1.8(S D=1.3)$ at baseline to $-0.9(S D=1.0)$ after 3 years of treatment $(P<0.01)$. The mean increase in BMI was 0.9 SDs $(S D=0.8)$.

Table 32: Height SDS and Height Velocity After Three Years of Treatment in the Polish Study on Increlex

\begin{tabular}{|l|c|}
\hline \multicolumn{2}{|c|}{$\begin{array}{c}\text { Polish Study on Increlex }(\mathrm{N}=25) \\
\text { Measures of height }\end{array}$} \\
\hline Height SDS, mean (SD) & $-3.5(0.8)$ \\
\hline Baseline & $-2.3(0.9)$ \\
\hline After 3 years of treatment & $<0.01$ \\
\hline P value (3 years) ${ }^{a}$ & \\
\hline Height velocity (cm/year), mean (SD) & $4.5(1.6)$ \\
\hline Baseline & $7.8(1.9)$ \\
\hline After 1 year of treatment & $6.3(0.8)$ \\
\hline After 2 years of treatment & $4.9(1.8)$ \\
\hline After 3 years of treatment & NA \\
\hline P value (3 years) & \\
\hline
\end{tabular}

$\mathrm{NA}=$ not available; $\mathrm{SD}=$ standard deviation .

aP value not adjusted for multiple comparisons.

Source: Petriczko et al. (2019). ${ }^{24}$ 


\section{Harms}

\section{Adverse Events}

Eight (30\%) patients reported AEs during the study. AEs included hypoglycemia $(n=2 ; 7 \%)$, hyperlipodystrophy at the injection site $(n=2 ; 7 \%)$, hypertrophy of the lymphatic tissue of the pharyngeal tonsils $(n=1 ; 4 \%)$, increase in scoliosis $(n=1 ; 4 \%)$, headache $(n=1 ; 4 \%)$, hair loss $(n=1 ; 4 \%)$, hearing loss due to chronic exudative otitis media $(n=1 ; 4 \%)$, and thickening of the ventricular septum in echocardiographic examination $(n=1 ; 4 \%)$.

\section{Serious Adverse Events}

The seriousness of AEs was not reported in the Polish Study on Increlex.

\section{Withdrawals Due to Adverse Events}

Two patients (7\%) discontinued the study due to AEs. One patient, who experienced thickening of the ventricular septum upon echocardiographic examination, was scheduled for heart valve implantation surgery and discontinued treatment after 30 months. The second patient, who experienced progression of scoliosis and conductive hearing loss due to chronic exudative otitis media, discontinued treatment after 24 months.

\section{Mortality}

No patient died during the study.

\section{Notable Harms}

Two patients (7\%) reported hypoglycemia during the sixth month of treatment. Two patients (7\%) had hyperlipodystrophy at the injection site. One patient (4\%) developed hypertrophy of the lymphatic tissue of the pharyngeal tonsils.

\section{Critical Appraisal Internal Validity}

The Polish Study on Increlex was a single-arm trial that investigated growth outcomes during the first 3 years of treatment among the first Polish patients treated with mecasermin for SPIGFD. Due to the rare and severe nature of SPIGFD, a randomized control group may not have been feasible, and the findings are therefore at high risk of confounding. The investigators did not control for known prognostic factors or effect modifiers (e.g., environmental or socioeconomic factors) within the analyses, and establishing a causal link between the treatment and the growth outcomes is not possible. The lack of a control group precludes making strong conclusions regarding the benefits and harms of treatment. The analyses were not adjusted for multiple comparisons, and there is therefore an increased risk of type I error.

All patients, their parents or caregivers, the treating clinicians, and the outcome assessors were aware of the prescribed treatment. Performance and detection bias are unlikely for growth outcomes as all were objectively measured. It is possible that common subjective harms known to be associated with mecasermin could have been over-reported, as patients and their treating clinicians knew of the treatment received and of their participation in a trial.

The study enrolled the first 27 children in Poland treated with mecasermin for SPIGFD and it does not appear that there is a substantial risk of selection bias. The power of the study is unclear; however, all Mann-Whitney $U$ tests to investigate changes from baseline were statistically significant. Only 2 patients (7\%) discontinued treatment early due to a reason 
other than the completion of their course of treatment. The missing data from these 2 patients are unlikely to have substantially biased the findings.

It is not clear how SDSs were calculated in the Polish Study on Increlex, as these details were not reported in the publication. For this reason, the validity of the outcomes based on SDSs is uncertain. As noted in previous discussions related to Study 1419 and the EU-IGFD Registry, results for outcomes based on SDSs can differ depending on the reference standard used.

There was no published protocol for the Polish Study on Increlex and the risk of bias due to selective reporting is high, largely because some clinically important outcomes were measured but not reported (i.e., bone age and bone age SDS). Final adult height among patients who achieved adult height during the study $(n=3 ; 11 \%)$ was also not reported.

\section{External Validity}

The Polish Study on Increlex included Polish patients treated with mecasermin for SPIGFD. The eligibility criteria were aligned with the product monograph ${ }^{12}$ : short stature below -3.0 SD for age and sex, and an IGF-1 concentration below the 2.5th percentile for age and sex (confirmed through nocturnal surge and/or simulation tests). Further, all patients underwent an IGF-1 generation test to assess for insensitivity to GH. Two (7\%) of the patients included in the study did not meet all eligibility criteria. One did not have a height lower than -3.0 SD for age and sex and the other did not have an IGF-1 concentration below the 2.5th percentile. One additional patient had a pre-treatment height velocity of $7.5 \mathrm{~cm}$ per year (although the patient still had a short stature, with a height SDS of -3.3). It is unlikely that the inclusion of these patients would substantially affect the generalizability of the findings.

The mean age at baseline was 10.1 years (range $=2.8$ to 16.2 years) and bone age was not reported. The majority (81\%) of patients were male. These patients would be eligible for treatment according to the product monograph based on age but are likely older than the optimal starting age of treatment. The results could have differed had patients started treatment sooner to optimize their response. Although SPIGFD affects males and females equally, the imbalance in the proportion of males and females enrolled is unlikely to affect the generalizability of the findings as the treatment is expected to have an equivalent effect on males and females.

The exposure to mecasermin in the Polish Study on Increlex is likely reflective of typical exposures for patients who would be treated in the Canadian context. All patients started mecasermin at a dosage of $40 \mathrm{mcg} / \mathrm{kg}$ SC twice daily, the recommended starting dosage in the product monograph. ${ }^{12} \mathrm{After} 12$ months, the average dosage increased to $80 \mathrm{mcg} /$ $\mathrm{kg} \mathrm{SC}$ twice daily. The average dosage after 3 years was $100 \mathrm{mcg} / \mathrm{kg} \mathrm{SC}$ twice daily (range $=80 \mathrm{mcg} / \mathrm{kg} \mathrm{SC}$ twice daily to $120 \mathrm{mcg} / \mathrm{kg}$ SC twice daily), which is aligned with the recommendations in the product monograph. ${ }^{12}$

\section{Discussion}

\section{Summary of Available Evidence}

This systematic review included evidence of the benefits and harms of mecasermin from 1 pivotal trial and 2 other relevant studies. The pivotal trial (Study 1419) was a phase III, 
multi-centre, single-arm, open-label trial with linked data from 4 predecessor studies.

Ninety-two patients with growth failure due to SPIGFD were enrolled, and measures of growth (height velocity, near-adult height, estimated improvement in adult height, height velocity SDS, height SDS, bone age relative to chronological age, and BMI SDS) were recorded annually and compared in paired analyses to baseline values for a maximum of 19 years. Harms but not HRQoL were measured. A historical (1993) cohort of patients with untreated Laron syndrome ${ }^{18}$ was used to estimate improvement in adult height. The findings for this outcome need to be interpreted cautiously as there is no evidence to support whether this historical cohort is representative of contemporary children with untreated SPIGFD. Otherwise, the interpretation of the findings from the pivotal trial is challenged by the lack of randomized control group, which may have been unfeasible or unethical due to the rare and severe nature of SPIGFD. Randomization controls for known and unknown confounders that could influence the treatment outcome and a control (placebo, untreated, or standard care) group allows for the estimation of the average effect of treatment in treated relative to untreated patients. The design of this single-arm trial precludes the inference of a causal relationship between mecasermin and growth and harms outcomes. The ability to draw conclusions was also limited because the analyses were not adjusted for multiple comparisons, creating an increased risk of type I error. Because the trial was open-label, there is also some risk that subjective harms could have been overestimated. A relatively large proportion of patients were lost to follow-up, resulting in a high risk of bias due to missing outcome data. Whether the study was powered to detect statistically significant changes from baseline (particularly at later time points when fewer patients remained in the study) is uncertain.

Two additional studies provided other relevant evidence, the EU-IGFD Registry and the Polish Study on Increlex. The EU-IGFD Registry enrolled 281 patients from 10 European countries treated with mecasermin for growth failure and reported on growth outcomes (height and height SDS, height velocity, weight and weight SDS, BMI and BMI SDS, bone age, predicted adult height, and final adult height) compared to baseline over a maximum of 10 years. Although HRQoL was measured, the data were gathered inconsistently, and measurements for baseline and some time point thereafter were only available for 2 patients. The Polish Study on Increlex enrolled the first 27 patients in Poland treated with mecasermin for SPIGFD and reported on height SDS, height velocity, weight SDS, and BMI SDS during the first 3 years of treatment. No HRQoL measurements were recorded. Both studies also reported on harms. Similar to Study 1419, neither of these studies included a randomized control group, undermining the ability to make meaningful inferences about the benefits and harms of treatment. The ability to draw conclusions was also limited because the analyses were not adjusted for multiple comparisons, increasing the risk of type I error. As with Study 1419, there is also some risk that subjective harms could have been overestimated. No sample size or power calculations were reported in either study; however, the EU-IGFD Registry reported that the enrolment target was not reached. Whether this study was powered to detect statistically significant changes from baseline is uncertain (particularly at later time points, when fewer patients remained in the study). The Polish Study on Increlex enrolled few patients; however, all reported changes from baseline values were statistically significant. In the EU-IGFD Registry, there is a high risk of bias for incomplete outcome data because a large proportion of enrolled patients discontinued the study early due to reasons other than completing the course of treatment or achieving final adult height, and baseline data were missing for many enrolled patients. In the Polish Study on Increlex, there is a high risk of reporting bias because some clinically important outcomes (e.g., bone age, bone age SDS, and final adult height) were measured but not reported. 
For the most part, the findings of the included studies appeared to be reasonably generalizable to Canadian patients. Study 1419 enrolled patients with less-severe short stature than indicated in the product monograph; however, all but 1 patient had a height SDS greater than -3.0 and all had genetically confirmed SPIGFD. The Polish Study on Increlex enrolled patients with confirmed SPIGFD as defined in the product monograph. The dosing regimen and concomitant therapies in both studies were aligned with the product monograph and Canadian clinical practice. The EU-IGFD Registry enrolled patients who were taking mecasermin for growth failure; however, only $15 \%$ of patients had Laron syndrome, which is the most common known cause of SPIGFD. The etiology of short stature among the remaining patients was not defined. Diagnostic criteria for starting mecasermin treatment were not described, and there was a wide range of baseline heights, some not meeting the minimum of $-3.0 \mathrm{SD}$ recommended in the product monograph. ${ }^{12}$ Otherwise, the dosing regimen was aligned with the product monograph ${ }^{12}$; however, a quarter of the patients experienced dosing interruptions or dosage decreases during the study due to mecasermin shortages.

\section{Interpretation of Results \\ Efficacy Outcomes \\ Height}

In Study 1419, the mean height velocity increased among naive-to-mecasermin patients during the first year of treatment. Height velocity remained significantly above baseline for the first 8 years of treatment, although the largest increase was observed during the first year. Height velocity SDSs increased from baseline during the first year of treatment and remained above baseline for 14 years of treatment, again with the most substantial gain during the first year. This finding is at risk of increased type I error because the analyses were not adjusted for multiple comparisons. The findings of Study 1419 were corroborated by those of the EU-IGFD Registry and the Polish Study on Increlex. According to the clinical expert consulted by CADTH, a substantial gain in height velocity during the first year of treatment followed by lesser but continued gains thereafter until final adult height was achieved aligns with the expected effect for patients with SPIGFD treated with mecasermin.

Owing to the design limitations of the included studies, it is not possible to determine with certainty the clinical significance of changes in height on treatment, and how the observed changes in height and height velocity would differ from untreated patients. The clinical expert consulted by CADTH nevertheless remarked that a height velocity of at least $2 \mathrm{~cm}$ per year (or $1 \mathrm{~cm}$ per 6 months) is generally considered sufficient to continue mecasermin treatment, by analogy with the clinical practice in patients treated with GH for GH deficiency. The median final adult height in Study 1419 was $137.6 \mathrm{~cm}$ (range $=112.0$ to 164.4). Despite treatment, the mean final adult height falls short of the global average of $159 \mathrm{~cm}$ for healthy females and $171 \mathrm{~cm}$ for healthy males, ${ }^{51}$ but is an estimated mean of $13 \mathrm{~cm}$ (range $=0$ to 35) taller than untreated patients with Laron syndrome who reach an average adult height of approximately $120 \mathrm{~cm}$ to $130 \mathrm{~cm}$ (mean of $124 \mathrm{~cm}$ [SD $=8.5]$ in males and $119 \mathrm{~cm}$ [SD $=8.5]$ in females). ${ }^{18}$

Given the rare and severe nature of SPIGFD, a randomized controlled trial may not have been feasible or ethical. Although single-arm trials and observational evidence do not allow for causal inferences, the outcomes for patients in the included studies, corroborated by those in other short-term trials, provide convincing evidence of a favourable effect of mecasermin on growth. Notably, the majority of patients in the pivotal trial demonstrated a clear response to mecasermin therapy in the form of a marked improvement in 1-year height 
velocity. ${ }^{15}$ The observed increase in height velocity is inconsistent with the usual growth trajectory in untreated patients with SPIGFD (for whom growth velocity remains relatively constant throughout the course of development). ${ }^{18}$ Similar height responses were shown in 2 randomized trials of rhIGF-1 therapy among patients with growth retardation (although not necessarily with confirmed SPIGFD) ${ }^{25,26}$ and a case-based study of 2 prepubertal siblings with PAPP-A2 homozygous frameshift gene mutation (an autosomal recessive syndrome consisting of short stature, among other symptoms). ${ }^{52}$ Acknowledging the limitations of the design while referencing the pivotal trial, the Pediatric Endocrine Society stated, "[...] the severe phenotype of untreated adults, the significant increase in (near-) adult height reported, the unanimous growth velocity acceleration compared to pre-treatment values in multiple short-term studies, and the lack of an alternative treatment make IGF-1 a strongly recommended treatment for growth failure in patients with primary insulin-like growth factor-1 deficiency [.... ${ }^{\prime \prime 14}$

\section{Health-Related Quality of Life}

As HRQoL was not reported in any of the studies included in the current review (it was investigated in the EU-IGFD Registry, but data were only available for 2 patients at baseline and some time point thereafter), the impact of mecasermin on HRQoL is unknown. The potential detrimental effect of twice-daily injections of mecasermin and other potential harms on HRQoL would need to be weighed against the uncertain potential for lifetime gains in HRQoL due to added height. Even if a patient's final height remains below average, it is possible that a gain in adult height could affect the daily lives of patients with SPIGFD. As noted by the patient group input submitted to CADTH, short stature is more than a cosmetic issue and can affect children's ability to participate in everyday life to the same extent as their healthy peers, and the potential benefit of treatment is "astronomical." Children living with short stature may have difficulty both in childhood and in their adult lives with daily activities, such as reaching for elevated objects and driving without modifications to a vehicle. ${ }^{53} \mathrm{~A} 2021$ systematic review of the burden of short stature in children and adults due to any cause ${ }^{31}$ found no studies reporting on HRQoL among patients with SPIGFD. According to the clinical expert consulted by CADTH, the value of gain in height among patients with SPIGFD with mecasermin treatment (independent of uncertain effects for other consequences of SPIGFD, which have not been shown in trials) is subjective.

\section{Skeletal Maturation}

Change in bone age relative to chronological age was assessed in Study 1419 and the EUIGFD Registry. In Study 1419, bone age was delayed at baseline relative to chronological age. Change in bone age exceeded the change in chronological age during treatment. Findings for the EU-IGFD Registry were similar, but the follow-up was substantially shorter, precluding the ability to draw conclusions about bone age progression over long-term treatment. These findings are uncertain because the analyses were not adjusted for multiple comparisons, increasing the risk of type I error. As there was no control group, it is not possible to determine whether the acceleration of bone age can be attributed to mecasermin treatment. The advancement of bone age relative to chronological age is an important consideration because bone age is 1 of the clinical criteria used to determine when treatment should cease. If treatment with mecasermin were to reduce the length of the growth period (i.e., by accelerating the advancement of bone age), then it is possible that the final adult height may not be taller than without treatment. 


\section{Body Mass or Composition}

All 3 studies measured BMI SDS and all reported an increase during treatment. In Study 1419, BMI SDS increased during treatment. Most patients had a BMI SDS between -2 and +2 , both when first and when last evaluated, or were nearly within this range. Among prepubertal naive-to-mecasermin patients in the EU-IGFD Registry and patients in the Polish Study on Increlex, the findings were similar. The findings are uncertain because the analyses were not adjusted for multiple comparisons, posing an increased risk of type I error. As there was no control group, it is not possible to determine whether the observed changes in BMI SDS can be attributed to mecasermin treatment. Increases in BMI SDS during treatment may be a result of mecasermin's insulin-like hypoglycemic effect, which requires each dose to be taken with a meal or snack. As height and weight are routinely measured in children being treated for SPIGFD, any excessive gains in weight and subsequent adjustments to treatment would likely need to be evaluated on a case-by-case basis.

\section{Harms}

The most frequently reported harms were similar across the included studies, although the Polish Study on Increlex reported fewer harm events overall compared with the other 2 studies. At least $1 \mathrm{AE}$ was reported by $83 \%$ of Study 1419 patients, $67 \%$ of those in the EU-IGFD Registry, and $30 \%$ of those in the Polish Study on Increlex. SAEs were reported by $20 \%$ of patients in Study 1419 and $21 \%$ in the EU-IGFD Registry. Very few patients stopped treatment due to AEs, and deaths during treatment were uncommon. The most common notable harms across the 3 studies were hypoglycemia, lipohypertrophy at the injection site, and tonsillar hypertrophy. As with the efficacy outcomes, design limitations of the included studies preclude making any strong conclusions about the relationship between mecasermin treatment and observed harms.

Hypoglycemia is a common side effect of mecasermin treatment ${ }^{12}$ and was the most common notable harm across all 3 studies. Measures can be taken to reduce the risk of hypoglycemia. Injections should always be provided within 30 minutes of a meal or snack and withheld if the patient is unable to eat. Further, patients are generally started on a lower dose and their blood glucose levels are closely monitored at the beginning of treatment. The dose may be temporarily reduced if hypoglycemia occurs. If tolerated, the dose is increased over time until the maximum is reached. Despite precautions, a large proportion of patients experienced hypoglycemia across the included studies. Hypoglycemic events were rarely severe and infrequently resulted in a change to therapy (e.g., treatment interruption or dose change) or discontinuation of treatment.

Lipohypertrophy at the injection site was the second most common notable harm across all 3 studies. To avoid lipohypertrophy, mecasermin injections sites should be rotated with each dose. In Study 1419, it was noted that cases of lipohypertrophy were associated with a lack of proper rotation of injection sites and resolved when injections were properly dispersed. Injection site lipohypertrophy was rarely severe and infrequently resulted in discontinuation of treatment.

The third most common notable harm across all 3 studies was tonsillar or adenoidal hypertrophy. A warning for lymphoid tissue hypertrophy, which can result in complications such as snoring, sleep apnea, and chronic middle-ear effusions, is provided in the product monograph. ${ }^{12}$ Tonsillar or adenoidal hypertrophy was rarely severe and infrequently resulted in discontinuation of treatment. 
Other warnings and precautions listed in the product monograph include benign and malignant neoplasms, slipped femoral epiphysis and progression of scoliosis (due to rapid growth), allergic reactions, and intracranial hypertension. These were infrequently reported across the included studies and rarely resulted in discontinuation of treatment.

\section{Other Considerations}

Complexity is inherent to the identification of patients who might benefit the most from mecasermin treatment as access to testing required for the definitive diagnosis of SPIGFD (e.g., GH antibody testing, genetic testing, or a clear test to assess IGF-1 response to GH [an IGF-1 generation test]) is limited in many Canadian jurisdictions. To reduce the risk of misdiagnosis and overtreatment, the clinical expert consulted by CADTH suggested that treatment decisions be made by a panel of clinical experts, at least when the diagnosis of SPIGFD is uncertain. This may not be feasible because few Canadian clinicians have direct experience with the diagnosis and treatment of SPIGFD; however, physicians with expertise in managing pediatric endocrine growth disorders may also be qualified to contribute.

The patient group consulted by CADTH emphasized that the health issues faced by patients with SPIGFD run deeper than just height and affect their entire lives. In addition to the appreciable uncertainty in the benefits of treatment with respect to growth outcomes, the included studies provided no evidence for the effect of mecasermin on other consequences of SPIGFD (e.g., metabolic abnormalities or muscle weakness). At present, therefore, the value of treatment hinges on the uncertain potential for gains in height, which must be weighed against equally uncertain potential for harm. A Canadian registry to monitor the benefits and long-term harms in patients treated with mecasermin may be useful.

\section{Conclusions}

One phase III, multi-centre, single-arm, open-label trial with linked data from 4 predecessor studies was included. Two other studies provided additional relevant evidence: 1 registry study of European patients and 1 single-arm trial in Poland. Due to the rare and severe nature of SPIGFD, a randomized control group was not feasible or ethical in the included trials, making it impossible to infer a causal relationship between mecasermin treatment and growth and harms outcomes. Nevertheless, data from the pivotal trial (Study 1419) appear to demonstrate a clear response to mecasermin therapy for most patients, as indicated by a marked improvement in 1-year height velocity that is not typical of untreated SPIGFD patients. ${ }^{18}$ These findings are corroborated by those of smaller, shorter-term randomized trials of recombinant IGF-1 therapy of patients with growth retardation. 25,26

Mecasermin treatment may improve final adult height in patients with SPIGFD compared with untreated patients with Laron syndrome. It is uncertain whether the historical control group used to support this conclusion is representative of contemporary untreated patients with SPIGFD. Of note, data from the pivotal trial showed that, during treatment, bone age advanced more quickly than chronological age and that BMI SDS increased. The implications of these changes need to be considered when evaluating the potential benefits of mecasermin; however, because the analyses were not controlled for multiple comparisons, there is an increased risk of type I error, and useful conclusions for these outcomes are limited. None of the studies provided usable data on HRQoL or the effects of mecasermin on other known health consequences of SPIGFD (e.g., metabolic abnormalities or muscle weakness). 
Harms were commonly reported across all studies, but serious harms were less frequent and deaths were rare. Few patients withdrew from any of the studies due to AEs. Among the most reported AEs were hypoglycemia, lipohypertrophy at the injection site, and tonsillar hypertrophy. For the most part, these AEs could be managed via careful monitoring and dosing changes or interruptions, and infrequently resulted in discontinuation of treatment. Other notable harms (e.g., benign or malignant neoplasia), were less frequent. Due to the challenges inherent in the identification of patients who might benefit most from mecasermin treatment, it may be helpful to engage a panel of clinical experts to inform treatment decisions, at least when the diagnosis cannot be confirmed. This may not be feasible as few Canadian clinicians have direct experience with the diagnosis and treatment of SPIGFD; however, physicians with expertise in managing pediatric endocrine growth disorders may also be qualified to contribute. A Canadian registry may be useful to monitor benefits and long-term harms in patients treated with mecasermin. 


\section{References}

1. Committee for Orphan Medicinal Products. Public summary of positive opinion for orphan designation of mecasermin for the treatment of primary insulin-like growth factor-1 deficiency due to molecular or genetic defects. Amsterdam (NL): European Medicine Agency; 2009: https://www.ema.europa.eu/en/documents/orphan -designation/eu/3/06/373-public-summary-positive-opinion-orphan-designation-mecasermin-treatment-primary-insulin-growth_en.pdf. Accessed 2021 Jul 16.

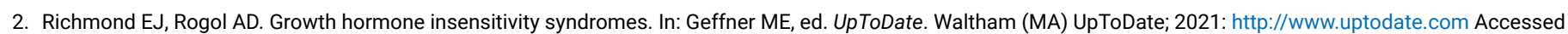
2021 Jul 16.

3. Clinical commissioning policy: mecasermin for treatment of growth failure. Leeds (GB): NHS Commissioning Board; 2013: https://www.england.nhs.uk/wp-content/ uploads/2018/07/Mecasermin-for-treatment-of-growth-failure.pdf. Accessed 2021 Jul 16.

4. Statistics Canada. Table 17-10-0016-01. Estimates of births, by sex, annual. 2020; https://www150.statcan.gc.ca/t1/tbl1/en/tv.action?pid=1710001601. Accessed $2021 \mathrm{Jul} 16$.

5. Statistics Canada. Table 17-10-0009-01. Population estimates, quarterly. 2021; https://www150.statcan.gc.ca/t1/tbl1/en/tv.action?pid=1710000901. Accessed 2021 Jul 16.

6. Disease prevalence and incidence [internal sponsor's report]. In: Drug Reimbursement Review sponsor submission: Increlex (mecasermin), 10mg/mL (40mg per vial) subcutaneous injection. Mississauga (ON): Ipsen Biopharmaceuticals Canada, Inc; 2020.

7. Laron Z, Pertzelan A, Mannheimer S. Genetic pituitary dwarfism with high serum concentation of growth hormone--a new inborn error of metabolism? Isr J Med Sci. 1966;2(2):152-155. PubMed

8. Laron Z. Lessons from 50 years of study of Laron syndrome. Endocr Pract. 2015;21(12):1395-1402. PubMed

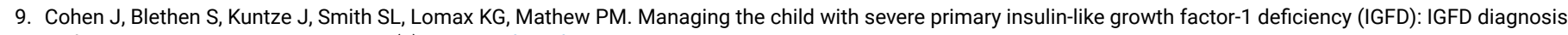
and management. Drugs R D. 2014;14(1):25-29. PubMed

10. Laron Z. Laron syndrome (primary growth hormone resistance or insensitivity): the personal experience 1958-2003. J Clin Endocrinol Metab. 2004;89(3):1031-1044. PubMed

11. Laron Z, Anin S, Klipper-Aurbach Y, Klinger B. Effects of insulin-like growth factor on linear growth, head circumference, and body fat in patients with Laron-type dwarfism. Lancet. 1992;339(8804):1258-1261. PubMed

12. Increlex (mecasermin): 10mg/mL (40mg per vial) injection [product monograph]. Mississauga (ON): Ipsen Biopharmaceuticals Canada, Inc; 2020 Dec 17.

13. Storr HL, Dunkel L, Kowalczyk J, Savage MO, Metherell LA. Genetic characterisation of a cohort of children clinically labelled as GH or IGF1 insensitive: diagnostic value of serum IGF1 and height at presentation. Eur J Endocrinol. 2015;172(2):151-161. PubMed

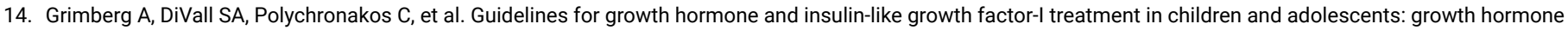
deficiency, idiopathic short stature, and primary insulin-like growth factor-I deficiency. Horm Res Paediatr. 2016;86(6):361-397. PubMed

15. Clinical Study Report: 1419. A study of long-term recombinant human insulin-like growth factor-1 (rhIGF-1) treatment of children with short stature due to severe primary IGF-1 deficiency study. This is an integrated study report involving five individual studies, F0206S, F0375G, F0632G, F0671G, and 1419 [internal sponsor's report]. Basking Ridge (NJ): Ipsen Biopharmaceuticals Inc; 2012 Jul 10.

16. Backeljauw PF, Kuntze J, Frane J, Calikoglu AS, Chernausek SD. Adult and near-adult height in patients with severe insulin-like growth factor-I deficiency after longterm therapy with recombinant human insulin-like growth factor-I. Horm Res Paediatr. 2013;80(1):47-56. PubMed

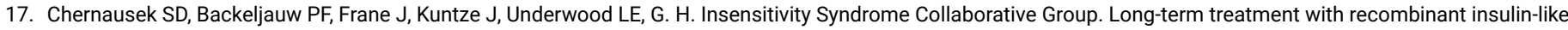
growth factor (IGF)-I in children with severe IGF-I deficiency due to growth hormone insensitivity. J Clin Endocrinol Metab. 2007;92(3):902-910. PubMed

18. Laron Z, Lilos P, Klinger B. Growth curves for Laron syndrome. Arch Dis Child. 1993;68(6):768-770. PubMed

19. Laron Z, Klinger B. Body fat in Laron syndrome patients: effect of insulin-like growth factor I treatment. Horm Res. 1993;40(1-3):16-22. PubMed

20. Clinical Study Report:2-79-52800-002. European Increlex (mecasermin [rDNA origin] injection) Growth Forum Database: a European subject registry for monitoring long-term safety and efficacy of Increlex - EU-IGFD [internal sponsor's report]. Boulogne Billancourt (FR): Ipsen Pharma SAS; 2020 Jan 10.

21. Bang P, Woelfle J, Perrot V, Sert C, Polak M. Effectiveness and safety of rhIGF1 therapy in patients with or without Laron syndrome. Eur J Endocrinol. 2021;184(2):267-276. PubMed

22. Bang P, Polak M, Woelfle J, Houchard A, Group EIRS. Effectiveness and safety of rhIGF-1 therapy in children: the European Increlex R Growth Forum Database experience. Horm Res Paediatr. 2015;83(5):345-357. PubMed

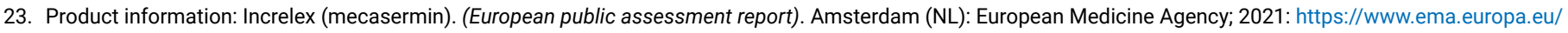
en/medicines/human/EPAR/increlex\#authorisation-details-section. Accessed 2021 Sep 27.

24. Petriczko E, Jackowski T, Horodnicka-Józwa A, et al. Treatment of severe primary IGF-1 deficiency using rhIGF-1 preparation - first three years of Polish experience. Endokrynol Pol. 2019;70(1):20-27. PubMed

25. Midyett LK, Rogol AD, Van Meter QL, Frane J, Bright GM, Group MSS. Recombinant insulin-like growth factor (IGF)-I treatment in short children with low IGF-I levels: first-year results from a randomized clinical trial. J Clin Endocrinol Metab. 2010;95(2):611-619. PubMed 


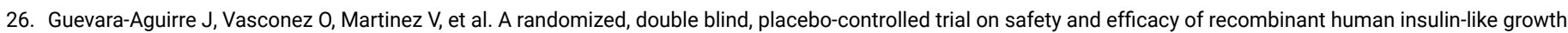
factor-I in children with growth hormone receptor deficiency. J Clin Endocrinol Metab. 1995;80(4):1393-1398. PubMed

27. U.S. Food and Drug Administration. Search orphan drug designations and approvals: mecasermin (Increlex). 2005; https://www.accessdata.fda.gov/scripts/ opdlisting/oopd/detailedlndex.cfm?cfgridkey=093695. Accessed $2021 \mathrm{Jul} 16$.

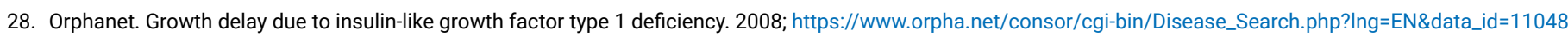
\&Disease_Disease_Search_diseaseGroup=Primary-insulin-like-growth-factor-deficiency\&Disease_Disease_Search_diseaseType=Pat\&Disease(s)/group\%20of\%20 diseases=Growth-delay-due-to-insulin-like-growth-factor-type-1-deficiency\&title=Growth\%20delay $\% 20$ due $\% 20$ to $\% 20$ insulin-like $\% 20$ growth $\% 20$ factor\%20type $\% 201 \% 20$ deficiency\&search=Disease_Search_Simple. Accessed 2021 Jul 16.

29. Savage MO. Phenotypes, investigation and treatment of primary IGF-1 deficiency. Endocr Dev. 2013;24:138-149. PubMed

30. Storr H. Recombinant IGF-1 therapy in children with severe primary IGF-1 deficiency (SPIGFD). London (GB): Centre for Endocrinology; 2018: https://www.bsped.org .uk/media/1464/uk-igf1-users-group-guidelines-20181.pdf. Accessed 2021 Jul 16.

31. Backeljauw P, Cappa M, Kiess W, et al. Impact of short stature on quality of life: a systematic literature review. Growth Horm IGF Res. 2021;57-58:101392. PubMed

32. Health Canada. Notice of compliance search results. Increlex. 2021; https://health-products.canada.ca/noc-ac/search-recherche.do;jsessionid=AA271E3B0695 C2CC0CA639E7BFF3AD00?lang=en. Accessed 2021 Jul 16.

33. McGowan J, Sampson M, Salzwedel DM, Cogo E, Foerster V, Lefebvre C. PRESS Peer Review of Electronic Search Strategies: 2015 guideline statement. J Clin Epidemiol. 2016;75:40-46. PubMed

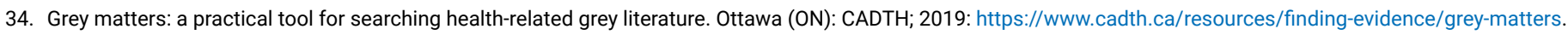
Accessed 2021 Jun 24.

35. Tanner JM, Whitehouse RH, Takaishi M. Standards from birth to maturity for height, weight, height velocity, and weight velocity: British children, 1965. II. Arch Dis Child. 1966;41(220):613-635. PubMed

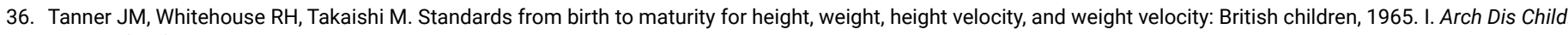
1966;41(219):454-471. PubMed

37. Kelly A, Winer KK, Kalkwarf H, et al. Age-based reference ranges for annual height velocity in US children. J Clin Endocrinol Metab. 2014;99(6):2104-2112. PubMed

38. Chumela WC, Roche AF, Thissen D. The FELS method of assessing the skeletal maturity of the hand-wrist. Am J Hum Biol. 1989;1(2):175-183. PubMed

39. Roche AF. Growth, maturation and body composition: the Fels Longitudinal Study 1929-1991. Cambridge (GB): Cambridge University Press; 1992.

40. Kuczmarski RJ, Ogden CL, Grummer-Strawn LM, et al. CDC growth charts: United States. Adv Data. 2000(314):1-27. PubMed

41. Kuczmarski RJ, Ogden CL, Guo SS, et al. 2000 CDC Growth Charts for the United States: methods and development. Vital Health Stat 11. 2002(246):1-190. PubMed

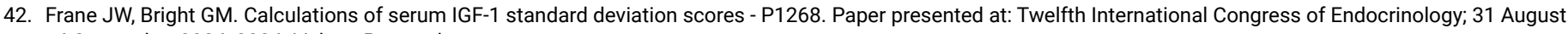
- 4 September 2004, 2004; Lisbon, Portugal.

43. Rodriguez-Martinez A, Zhou B, Sophiea MK, et al. Height and body-mass index trajectories of school-aged children and adolescents from 1985 to 2019 in 200 countries and territories: a pooled analysis of 2181 population-based studies with 65 million participants. The Lancet. 2020;396(10261):1511-1524. PubMed

44. WHO Multicentre Growth Reference Study Group. WHO Child Growth Standards based on length/height, weight and age. Acta Pædiatrica. 2006;450 (suppl):76-85. PubMed

45. Canadian Pediatric Endocrine Group. WHO growth charts for Canada. 2014; https://www.cpeg-gcep.net/content/who-growth-charts-canada. Accessed 2021 Aug 9.

46. Sempé M, Pédron G, Roy-Pernot M-P. Auxologie: méthode et séquences. Paris (Fr): Théraplix; 1979.

47. Hercberg S, Chat-Yung S, Chauliac M. The French National Nutrition and Health Program: 2001-2006-2010. Int J Public Health. 2008;53(2):68-77. PubMed

48. Wright CM, Booth IW, Buckler JMH, et al. Growth reference charts for use in the United Kingdom. Arch Dis Child. 2002;86(1):11. PubMed

49. Hermanussen $\mathrm{M}$, Assmann $\mathrm{C}$, Wöhling $\mathrm{H}$, Zabransky $\mathrm{M}$. Harmonizing national growth references for multi-centre surveys, drug monitoring and international postmarketing surveillance. Acta Paediatr. 2012;101(1):78-84. PubMed

50. Kêkê LM, Samouda H, Jacobs J, et al. Body mass index and childhood obesity classification systems: a comparison of the French, International Obesity Task Force (IOTF) and World Health Organization (WHO) references. Rev Epidemiol Sante Publique. 2015;63(3):173-182. PubMed

51. Roser M, Appel C, Ritchie H, Our World in Data. Human Height. 2019; https://ourworldindata.org/human-height. Accessed 2021 Aug 18.

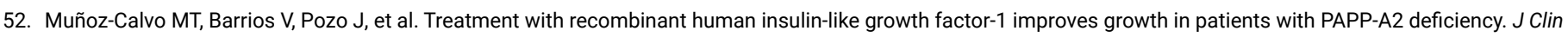
Endocrinol Metab. 2016;101(11):3879-3883. PubMed

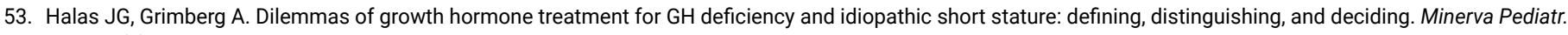
2020;72(3):206-225. PubMed 


\section{Appendix 1: Literature Search Strategy}

Note that this appendix has not been copy-edited.

\section{Clinical Literature Search}

Overview

Interface: Ovid

Databases

- MEDLINE All (1946-present)

- Embase (1974-present)

- Note: Subject headings and search fields have been customized for each database. Duplicates between databases were removed in Ovid.

Date of search: July 13, 2021

Alerts: Bi-weekly search updates until project completion

Search filters applied: No filters were applied to limit the retrieval by study type.

Limits

- No date or language limits were used

- Conference abstracts: excluded

\section{Table 33: Syntax Guide}

\begin{tabular}{|c|c|}
\hline Syntax & Description \\
\hline / & At the end of a phrase, searches the phrase as a subject heading \\
\hline $\mathrm{MeSH}$ & Medical Subject Heading \\
\hline $\exp$ & Explode a subject heading \\
\hline * & $\begin{array}{l}\text { Before a word, indicates that the marked subject heading is a primary topic; or, after a word, a truncation symbol } \\
\text { (wildcard) to retrieve plurals or varying endings }\end{array}$ \\
\hline ti & Title \\
\hline .ot & Original title \\
\hline.$a b$ & Abstract \\
\hline hw & Heading word; usually includes subject headings and controlled vocabulary \\
\hline.$k f$ & Author keyword heading word (MEDLINE) \\
\hline.$k w$ & Author keyword (Embase) \\
\hline. $\mathrm{dq}$ & Candidate term word (Embase) \\
\hline.$r n$ & Registry number \\
\hline. $\mathrm{nm}$ & Name of substance word (MEDLINE) \\
\hline medall & Ovid database code: MEDLINE All, 1946 to present, updated daily \\
\hline
\end{tabular}




\begin{tabular}{|l|l|}
\hline Syntax & \multicolumn{1}{c|}{ Description } \\
\hline oemezd & Ovid database code; Embase, 1974 to present, updated daily \\
\hline
\end{tabular}

\section{Multi-Database Strategy}

1. (mecasermin* or Increlex* or CEP 151 or CEP151 or myotrophin* or somazon* or Human somatomedin C or rhIGF-1 or recombinant somatomedin C or Somatomedin-1 or FK-780 or CG-GF2 or CG-IGF-1 or PV-802 or PV802 or 7GR9I26830). $\mathrm{ti}, \mathrm{ab}, \mathrm{kf}, \mathrm{ot}, \mathrm{hw}, \mathrm{rn}, \mathrm{nm}$.

2. (recombinant adj3 (insulin like growth factor1 or IGF1 or IGF-1 or IGF1A or IGFI or MGF or IGF)).ti,ab,kf,ot,hw.

3. 1 or 2

4. 3 use medall

5. *recombinant somatomedin $\mathrm{C} /$

6. (mecasermin* or Increlex* or CEP 151 or CEP151 or myotrophin* or somazon* or Human somatomedin C or rhIGF-1 or recombinant somatomedin C or Somatomedin-1 or FK-780 or CG-GF2 or CG-IGF-1 or PV-802 or PV802).ti,ab,kw,dq.

7. (recombinant adj3 (insulin like growth factor1 or IGF1 or IGF-1 or IGF1A or IGFI or MGF or IGF)).ti,ab,kw,dq.

8. or/5-7

9. 8 use oemezd

10. 9 not (conference review or conference abstract).pt.

11. 4 or 10

12. (Randomized Controlled Trial or Controlled Clinical Trial or Pragmatic Clinical Trial or Equivalence Trial or Clinical Trial, Phase III).pt.

13. Randomized Controlled Trial/

14. exp Randomized Controlled Trials as Topic/

15. "Randomized Controlled Trial (topic)"/

16. Controlled Clinical Trial/

17. exp Controlled Clinical Trials as Topic/

18. "Controlled Clinical Trial (topic)"/

19. Randomization/

20. Random Allocation/

21. Double-Blind Method/

22. Double Blind Procedure/

23. Double-Blind Studies/

24. Single-Blind Method/

25. Single Blind Procedure/

26. Single-Blind Studies/

27. Placebos/

28. Placebo/ 
29. Control Groups/

30. Control Group/

31. (random* or sham or placebo*).ti,ab,hw,kf,kw.

32. ((sing $\left.\right|^{\star}$ or doubl*) adj (blind* or dumm* or mask*)).ti,ab,hw,kf,kw.

33. ((trip ${ }^{\star}$ or trebl*) adj (blind* or dumm* or mask*)).ti,ab,hw,kf,kw.

34. (control* adj3 (study or studies or trial* or group*)).ti,ab,kf,kw.

35. (Nonrandom* or non random* or non-random* or quasi-random* or quasirandom*).ti,ab,hw,kf,kw.

36. allocated.ti,ab,hw.

37. ((open label or open-label) adj5 (study or studies or trial*)).ti,ab,hw,kf,kw.

38. ((equivalence or superiority or non-inferiority or noninferiority) adj3 (study or studies or trial*)).ti,ab,hw,kf,kw.

39. (pragmatic study or pragmatic studies).ti,ab,hw,kf,kw.

40. ((pragmatic or practical) adj3 trial*).ti,ab,hw,kf,kw.

41. ((quasiexperimental or quasi-experimental) adj3 (study or studies or trial*)).ti,ab,hw,kf,kw.

42. (phase adj3 (III or "3") adj3 (study or studies or trial*)).ti,hw,kf,kw.

43. or/12-42

44. 11 and 43

45. exp animals/

46. exp animal experimentation/ or exp animal experiment/

47. exp models animal/

48. nonhuman/

49. exp vertebrate/ or exp vertebrates/

50. or/45-49

51. exp humans/

52. exp human experimentation/ or exp human experiment/

53. or/51-52

54.50 not 53

55. 44 not 54

56. remove duplicates from 55

\section{Clinical Trials Registries}

ClinicalTrials.gov

Produced by the US National Library of Medicine. Targeted search used to capture registered clinical trials.

[Search -- Studies with results | Increlex OR mecasermin] 


\section{Health Canada's Clinical Trials Database}

Produced by Health Canada. Targeted search used to capture registered clinical trials.

[Search terms -- Increlex OR mecasermin]

\section{EU Clinical Trials Register}

European Union Clinical Trials Register, produced by the European Union. Targeted search used to capture registered clinical trials.

[Search terms -- Increlex OR mecasermin]

\section{Grey Literature}

Search dates: June 29, 2021-July 7, 2021

Keywords: [Provide terms used in grey literature search]

Limits: Publication years: 1996-present

Updated: Search updated prior to the completion of stakeholder feedback period

Relevant websites from the following sections of the CADTH grey literature checklist Grey Matters: A Practical Tool for Searching Health-Related Grey Literature were searched:

- Health Technology Assessment Agencies

- Health Economics

- Clinical Practice Guidelines

- Drug and Device Regulatory Approvals

- Advisories and Warnings

- Drug Class Reviews

- Clinical Trials Registries

- Databases (free)

- Health Statistics

- Internet Search 


\section{Appendix 2: Excluded Studies}

Note that this appendix has not been copy-edited.

\section{Table 34: Excluded Studies}

\begin{tabular}{|c|c|}
\hline Reference & Reason for exclusion \\
\hline $\begin{array}{l}\text { Backeljauw PF, Miller BS, Dutailly } \mathrm{P} \text {, et al. Recombinant human growth hormone plus } \\
\text { recombinant human insulin-like growth factor- } 1 \text { coadministration therapy in short children } \\
\text { with low insulin-like growth factor- } 1 \text { and growth hormone sufficiency: results from a } \\
\text { randomized, multicenter, open-label, parallel-group, active treatment-controlled trial. Horm } \\
\text { Res Paediatr. } 2015 ; 83(4): 268-279 \text {. }\end{array}$ & Population \\
\hline $\begin{array}{l}\text { Backeljauw PF, Miller BS, Dutailly } \mathrm{P} \text {, et al. Recombinant human growth hormone plus } \\
\text { recombinant human insulin-like growth factor- } 1 \text { coadministration therapy in short children } \\
\text { with low insulin-like growth factor-1 and growth hormone sufficiency: results from a } \\
\text { randomized, multicenter, open-label, parallel-group, active treatment-controlled trial. Horm } \\
\text { Res Paediatr. } 2015 ; 83(4): 268-279 \text {. }\end{array}$ & Duplicate \\
\hline $\begin{array}{l}\text { Bang P, Polak M, Woelfle J, Houchard A, Group EIRS. Effectiveness and Safety of rhIGF-1 } \\
\text { Therapy in Children: The European Increlex R Growth Forum Database Experience. Horm Res } \\
\text { Paediatr. } 2015 ; 83(5): 345-357 .\end{array}$ & Study designa \\
\hline $\begin{array}{l}\text { Bang P, Polak M, Woelfle J, Houchard A, Group EIRS. Effectiveness and Safety of rhIGF-1 } \\
\text { Therapy in Children: The European Increlex R Growth Forum Database Experience. Horm Res } \\
\text { Paediatr. } 2015 ; 83(5): 345-357 .\end{array}$ & Duplicate $^{\mathrm{a}}$ \\
\hline $\begin{array}{l}\text { Bang P, Woelfle J, Perrot V, Sert C, Polak M. Effectiveness and safety of rhIGF1 therapy } \\
\text { in patients with or without Laron syndrome. European Journal of Endocrinology. } \\
2021 ; 184(2): 267-276 .\end{array}$ & Study designa \\
\hline $\begin{array}{l}\text { Chernausek SD, Backeljauw PF, Frane J, Kuntze J, Underwood LE, Group GHISC. Long-term } \\
\text { treatment with recombinant insulin-like growth factor (IGF)-I in children with severe IGF-I } \\
\text { deficiency due to growth hormone insensitivity. J Clin Endocrinol Metab. 2007;92(3):902-910. }\end{array}$ & Duplicate \\
\hline $\begin{array}{l}\text { Clinical Study Report: MS301. Recombinant human insulin-like growth factor-1 (rhIGF-1) } \\
\text { treatment of prepubertal children with growth failure associated with primary IGF-1 } \\
\text { deficiency: a phase 3, randomized, open label, observation-controlled, multicenter, parallel- } \\
\text { dose comparison trial [CONFIDENTIAL internal manufacturer's report]. Brisbane, California, } \\
\text { USA: Tercica Inc; } 19 \text { April 2011. }\end{array}$ & Population \\
\hline $\begin{array}{l}\text { Clinical Study Report: MS305. Increlex(R) (mecasermin (rDNA origin) injection) growth forum } \\
\text { database-IGFD registry, a patient registry for monitoring long-term safety and effectiveness } \\
\text { of Increlex [CONFIDENTIAL internal manufacturer's report]. Basking Ridge, New Jersey: Ipsen } \\
\text { Biopharmaceuticals, Inc; } 16 \text { September } 2014 \text {. }\end{array}$ & Population \\
\hline $\begin{array}{l}\text { Clinical Study Report: MS306. Recombinant human insulin-like growth factor-1 (rhIGF-1) } \\
\text { treatment of children and adolescents with growth failure associated with primary IGF-1 } \\
\text { deficiency: an open-label, multi-center, extension study (abbreviated report) [CONFIDENTIAL } \\
\text { internal manufacturer's report]. Brisbane, California, USA: Ipsen US; } 14 \text { October } 2011 .\end{array}$ & Population \\
\hline $\begin{array}{l}\text { Clinical Study Report: MS308. Recombinant human insulin-like growth factor-1 (rhIGF-1) } \\
\text { treatment of short stature associated with primary IGF-1 deficiency: a multicenter, open-label, } \\
\text { concentration-controlled trial [CONFIDENTIAL internal manufacturer's report]. Brisbane, } \\
\text { California, USA: Tercica Inc; } 30 \text { November } 2009 .\end{array}$ & Population \\
\hline
\end{tabular}


Clinical Study Report: MS316. Recombinant human growth hormone (rhGH) and recombinant human insulin-like growth factor-1 (rhIGF-1) combination therapy in children with short stature associated with IGF-1 deficiency: a six-year, randomized, multi-center, open label, parallel-group, active treatment controlled, dose selection trial [CONFIDENTIAL internal manufacturer's report]. Basking Ridge, New Jersey, USA: Ipsen Biopharmaceuticals Inc; 30 August 2012.

Clinical Study Report: EU-IGFD. European Increlex(R) (mecasermin [rDNA origin] injection) growth forum database: a European subject registry for monitoring long-term safety and effectiveness of Increlex(r) - EU-IGFD [CONFIDENTIAL internal manufacturer's report]. Boulogne Billancourt, France: Ipsen Pharma SAS; 10 January 2020.

Grahnen A, Kastrup K, Heinrich U, et al. Pharmacokinetics of recombinant human insulinlike growth factor I given subcutaneously to healthy volunteers and to patients with growth hormone receptor deficiency. Acta Paediatrica, International Journal of Paediatrics, Supplement. 1993;82(391):9-13.

Guevara-Aguirre J, Guevara A, Guevara C. Treatment of growth failure in the absence of GH signalling: The Ecuadorian experience. Growth Horm IGF Res. 2018;38:53-56.

Guevara-Aguirre J, Rosenbloom AL, Guevara-Aguirre M, Saavedra J, Procel P. Recommended IGF-I dosage causes greater fat accumulation and osseous maturation than lower dosage and may compromise long-term growth effects. J Clin Endocrinol Metab. 2013;98(2):839-845.

Guevara-Aguirre J, Rosenbloom AL, Vasconez O, et al. Two-year treatment of growth hormone $(\mathrm{GH})$ receptor deficiency with recombinant insulin-like growth factor I in 22 children: Comparison of two dosage levels and to GH-treated GH deficiency. J Clin Endocrinol Metab. 1997;82(2):629-633.

Guevara-Aguirre J, Vasconez O, Martinez V, et al. A randomized, double blind, placebocontrolled trial on safety and effectiveness of recombinant human insulin-like growth factor-I in children with growth hormone receptor deficiency. J Clin Endocrinol Metab. 1995;80(4):1393-1398.

Klinger B, Laron Z. Three year IGF-I treatment of children with Laron syndrome. J Pediatr Endocrinol Metab. 1995;8(3):149-158.

Laron Z, Silbergeld A, Lilos P, Blum FWF. Serum leptin in obese patients with Laron syndrome before and during IGF-I treatment. J Pediatr Endocrinol Metab. 1998;11(5):653-656.

Laron Z, Suikkari AM, Klinger B, et al. Growth hormone and insulin-like growth factor regulate insulin-like growth factor-binding protein-1 in Laron type dwarfism, growth hormone deficiency and constitutional short stature. Acta Endocrinol (Copenh). 1992;127(4):351-358.

Midyett LK, Rogol AD, Van Meter QL, Frane J, Bright GM, Group MSS. Recombinant insulin-like growth factor (IGF)-I treatment in short children with low IGF-I levels: first-year results from a randomized clinical trial. J Clin Endocrinol Metab. 2010;95(2):611-619.

Midyett LK, Rogol AD, Van Meter QL, Frane J, Bright GM, Group MSS. Recombinant insulin-like growth factor (IGF)-I treatment in short children with low IGF-I levels: first-year results from a randomized clinical trial. J Clin Endocrinol Metab. 2010;95(2):611-619.

Petriczko E, Jackowski T, Horodnicka-Józwa A, et al. Treatment of severe primary IGF-1 deficiency using rhIGF-1 preparation - first three years of Polish experience. Endokrynol Pol. 2019;70(1):20-27.

Population

Study design a

Population

Study design

Study design

Comparator

Population

Study design

Study design

Study design

Population

Duplicate

Study designa 
Ranke MB, Savage MO, Chatelain PG, Preece MA, Rosenfeld RG, Wilton P. Long-term

Study design treatment of growth hormone insensitivity syndrome with IGF-I. Results of the European Multicentre Study. Horm Res. 1999;51(3):128-134.

Wilson KF, Fielder PJ, Guevara-Aguirre J, et al. Long-term effects of insulin-like growth factor (IGF)-I treatment on serum IGFs and IGF binding proteins in adolescent patients with growth hormone receptor deficiency. Clin Endocrinol (Oxf). 1995;42(4):399-407.

Study design

ancluded in the Other Relevant Evidence section of this report. 


\section{List of Tables}

Table 1: Submitted for Review 112

Table 2: Summary of Economic Evaluation .112

Table 3: Summary of the Sponsor's Economic Evaluation Results 118

Table 4: Key Assumptions of the Submitted Economic Evaluation (Not Noted as Limitations to the Submission).

Table 5: CADTH Revisions to the Submitted Economic Evaluation

Table 6: Summary of the Stepped Analysis of the CADTH Reanalysis Results

Table 7: CADTH Price-Reduction Analyses

Table 8: CADTH Cost Comparison Table for Growth Failure in Children and Adolescents With Confirmed Severe Primary Insulin-Like Growth Factor 1 Deficiency

Table 9: Submission Quality 128

Table 10: Disaggregated Summary of CADTH's Economic Evaluation Results .130

Table 11: Disaggregated Summary of CADTH's Economic Evaluation Results Weighted by Proportion of Patients by Age of Treatment Initiation

Table 12: Scenario Analyses for Mecasermin vs. No Treatment. .131

Table 13: Summary of Key Take-Aways...... 132

Table 14: Summary of Key Model Parameters...... 133

Table 15: CADTH Revisions to the Submitted Budget Impact Analysis 134

Table 16: Summary of the CADTH Reanalyses of the Budget Impact Analysis .134

Table 17: Detailed Breakdown of the CADTH Reanalyses of the Budget Impact Analysis .135

Table 18: CADTH Scenario Analyses. .135 


\title{
Abbreviations
}

\author{
BIA budget impact analysis \\ BMI body mass index \\ EQ-5D-3L EQ-5D 3-Levels questionnaire \\ HRQoL health-related quality of life \\ ICER incremental cost-effectiveness ratio \\ QALY quality-adjusted life-year \\ SDS standard deviation score \\ SPIGFD severe primary insulin-like growth factor 1 deficiency
}




\section{Executive Summary}

The executive summary comprises 2 tables (Table 1 and Table 2 ) and a conclusion.

\section{Table 1: Submitted for Review}

\begin{tabular}{|l|l|}
\hline Item & \multicolumn{1}{|c|}{ Description } \\
\hline Drug product & Mecasermin (Increlex), subcutaneous injection \\
\hline Submitted price & Mecasermin, $40 \mathrm{mg}$ vial, \$5,916.64 per vial (\$147.92 per mg) \\
\hline Indication & $\begin{array}{l}\text { For the treatment of growth failure in children and adolescents from 2 to 18 years of age with } \\
\text { confirmed severe primary insulin-like growth factor-1 deficiency }\end{array}$ \\
\hline Health Canada approval status & NOC \\
\hline Health Canada review pathway & Standard \\
\hline NOC date & December 17, 2020 \\
\hline Reimbursement request & As per indication \\
\hline Sponsor & Ipsen Biopharmaceuticals Canada, Inc. \\
\hline Submission history & Previously reviewed: No \\
\hline
\end{tabular}

$\mathrm{NOC}=$ Notice of Compliance

\section{Table 2: Summary of Economic Evaluation}

\begin{tabular}{|l|l|}
\hline Component & \multicolumn{1}{|c|}{ Description } \\
\hline Type of economic evaluation & $\begin{array}{l}\text { Cost-utility analysis } \\
\text { Markov model }\end{array}$ \\
\hline Target population & $\begin{array}{l}\text { Children and adolescents from 2 to 18 years of age with confirmed severe primary insulin-like } \\
\text { growth factor-1 deficiency }\end{array}$ \\
\hline Treatment & Mecasermin \\
\hline Comparator & No treatment \\
\hline Perspective & Canadian publicly funded health care payer \\
\hline Outcome & QALYs \\
\hline Time horizon & $\begin{array}{l}\text { Lifetime (up to a patient's average life expectancy of 79.9 years for males and 84.1 years for } \\
\text { females) }\end{array}$ \\
\hline Key data source & $\begin{array}{l}\text { Treatment effectiveness with mecasermin was informed by an investigator-sponsored, single- } \\
\text { arm, open-label trial } \\
\text { Natural history data for untreated patients was sourced from a study in the literature } \\
\text { describing annual height velocities for children with Laron syndrome }\end{array}$ \\
\hline Submitted results & $\begin{array}{l}\text { ICER }=\$ 391,879 \text { per QALY gained (incremental costs: \$2,330,629; incremental QALYs: } 5.9 \text { ) } \\
\text { ICER weighted by sex and age of treatment initiation, with starting ages ranging from 2 to } 5 \\
\text { years }\end{array}$ \\
\hline
\end{tabular}




\begin{tabular}{|c|c|}
\hline Component & Description \\
\hline Key limitations & $\begin{array}{l}\text { - The clinical evidence available for mecasermin was from a single-arm trial. As a result, } \\
\text { the comparative clinical efficacy and safety of mecasermin compared with no treatment } \\
\text { is highly uncertain. The sponsor incorporated treatment effects into the model via a naive } \\
\text { comparison of mecasermin and no treatment; as a result, the model predictions of gains in } \\
\text { height with mecasermin are uncertain. } \\
\text { - The sponsor's model base case predicts a large QALY gain with mecasermin based on an } \\
\text { average gain in height of } 12.5 \mathrm{~cm} \text {. The association between height gain and utility scores in } \\
\text { patients with SPIGFD has not been established. There are also concerns with the sponsor's } \\
\text { approach to extrapolating utility scores by height SDS in the general adult population in the } \\
\text { absence of available data. } \\
\text { - The trial informing treatment efficacy of mecasermin in the sponsor's submitted model } \\
\text { excluded patients without a genetic cause of SPIGFD. The generalizability of the sponsor's } \\
\text { economic model results to such patients is uncertain. } \\
\text { - A greater proportion of males than females in the modelled population were assumed to } \\
\text { have SPIGFD, and, as such, the sex distribution of the modelled patient population used } \\
\text { to weight model results was not truly reflective of patients with SPIGFD. Given ICERs were } \\
\text { higher in females, this biased results in favour of mecasermin. } \\
\text { - Drug costs may be underestimated due to the exclusion of potential wastage. }\end{array}$ \\
\hline CADTH reanalysis results & $\begin{array}{l}\text { - Due to the significant uncertainty associated with the comparative clinical efficacy and } \\
\text { safety evidence, as well as with the patient utility by height SDS, CADTH produced a best } \\
\text { estimate rather than a base case. A base case was considered inappropriate given the } \\
\text { considerable uncertainty with the available evidence informing the model. The CADTH } \\
\text { best estimate included: revising health-state utilities to reflect the sponsor's extrapolation } \\
\text { with the least-severe decline in utility scores for a height SDS less than - } 3.5 \text { in the absence } \\
\text { of more appropriate evidence; assuming an equal proportion of males and females with } \\
\text { SPIGFD; and adjusting drug cost calculations to account for potential wastage. } \\
\text { - Based on the CADTH reanalyses, the ICER for mecasermin vs. no treatment was } \$ 624,249 \\
\text { per QALY gained (incremental costs: } \$ 2,338,189 \text {; incremental QALYs: } 3.8) \text {. A } 92 \% \text { price } \\
\text { reduction would be required for mecasermin to be considered cost-effective at a } \$ 50,000 \\
\text { per QALY threshold. } \\
\text { - The results remain highly uncertain due to limitations with the comparative efficacy of } \\
\text { mecasermin vs. no treatment and rely on the assumption that a gain of } 11.8 \mathrm{~cm} \text { in height } \\
\text { (as predicted in the CADTH best estimate) would result in approximately } 4 \text { additional years } \\
\text { of perfect health over a patient's lifetime. }\end{array}$ \\
\hline
\end{tabular}

ICER = incremental cost-effectiveness ratio; QALY = quality-adjusted life-year; SDS = standard deviation score; SPIGFD = severe primary insulin-like growth factor 1 deficiency; vs. = versus .

\section{Conclusions}

The clinical evidence submitted by the sponsor demonstrated that, in pediatric patients with severe primary insulin-like growth factor-1 deficiency (SPIGFD), treatment with mecasermin may improve final adult height compared with untreated patients with Laron syndrome. Due to the lack of a randomized control group in the pivotal trial, it was not possible to infer a causal relationship between mecasermin treatment and growth and harms outcomes and, as such, the comparative clinical efficacy or safety of mecasermin compared to no treatment remains unknown.

Due to the lack of available comparative clinical efficacy and safety data, as well as uncertainty in the impact of a $12.5 \mathrm{~cm}$ gain in height on patient quality of life over their lifetime, CADTH reanalysis consisted of a best estimate rather than a base case. CADTH's 
best-estimate reanalysis included revising health-state utilities to reflect the sponsor's extrapolation with the least-severe decline in utility scores using a height standard deviation score (SDS) of less than -3.5 from the sponsor's model in the absence of available data; assuming an equal proportion of males and females to better align with the distribution in Canadian clinical practice; and adjusting drug cost calculations to account for wastage. The CADTH best-estimate results were aligned with the results reported by the sponsor as mecasermin was not cost-effective at conventional willingness-to-pay thresholds. In the CADTH best estimate, mecasermin was associated with an incremental cost-effectiveness ratio (ICER) of $\$ 624,249$ per quality-adjusted life-year (QALY) gained (incremental costs of $\$ 2,338,189$ and an incremental benefit of 3.8 QALYs) compared with no treatment. A price reduction of at least $92 \%$ would be required for mecasermin to be considered cost-effective at a willingness-to-pay threshold of $\$ 50,000$ per QALY gained.

At the sponsor-submitted price, mecasermin is not cost-effective and uncertainty remains with regards to the magnitude of benefit associated with the gains in height predicted by the sponsor's model. The economic model predicted a gain in final height of $11.8 \mathrm{~cm}$ for patients treated with mecasermin in comparison with no treatment, resulting in a gain of 3.8 QALYS, or nearly 4 additional years in perfect health in the CADTH best-estimate analysis. Given the uncertainty in both the predicted gain in height and the impact of gains in height on patient utility scores, the QALY gains are highly uncertain and the ICER for mecasermin in comparison with no treatment is potentially underestimated. While there is uncertainty regarding the clinical benefits of mecasermin, the drug acquisition costs of mecasermin over the patient's lifetime $(\$ 2,335,609$ incremental drug costs as predicted by the model) are not associated with the same level of uncertainty.

\section{Stakeholder Input Relevant to the Economic Review}

This section is a summary of the feedback received from the patient groups and drug plans that participated in the CADTH review process, and information that pertains to the economic submission specifically. Clinician group input was not received.

One patient group, the International Coalition of Organizations Supporting Endocrine Patients, provided input. Patient input indicated the importance of diagnosing and treating children early to reduce additional medical hardships that may occur throughout their lifetime. Patient input indicated that children with SPIGFD experience significant chronic health issues beyond reduced height and have a very poor baseline health status. While short stature is a visual indicator of SPIGFD, it is related to several other clinical manifestations among untreated patients, such as reduced heart strength and lung capacity, that can result in lifelong damage, and it should not be understood as a cosmetic issue related to growth. Patient input reported that activities of daily living and mental tasks are effortful, and patients may lack the energy required to function adequately. Patient input noted that there are currently no available treatment options for SPIGFD and emphasized that, without treatment, patients are unlikely to improve given the natural history of this disease.

Feedback from the drug plans indicated that there are currently no available treatment options for the indicated population. Drug plans are unclear as to whether mecasermin provides additional benefits to the indicated population for several other important clinical manifestations beyond short stature. Drug plans questioned at which point over the treatment 
period should mecasermin be discontinued: after 8 years based on the mean follow-up from the clinical trials, after closure of epiphyseal plates, or up until the chronological age of 18 years (given that mecasermin is not indicated for patients beyond 18 years of age)? Drug plans raised concerns about the role of concomitant medications or prior therapies that may be required by patients. Drug plans also identified additional concerns pertaining to the costeffectiveness of mecasermin given the potential benefits (i.e., increased height) and harms of mecasermin (i.e., benign or malignant neoplasms) and the management of these adverse effects among treated patients. Finally, drug plans were concerned about the anticipated budget impact of reimbursing mecasermin.

Several of these concerns were addressed in the sponsor's model:

- The sponsor's base-case analysis appropriately compared mecasermin to no treatment, which is reflective of current clinical practice based on the patient and drug plan input.

- Health-related quality of life (HRQOL) was captured by the utility benefit associated with gains in height.

- The sponsor captured treatment discontinuation in its base-case analysis based on the rates of treatment discontinuation in the pivotal trial, which accounted for treatment discontinuation due to noncompliance, parent or patient decision, and poor growth per year.

- The sponsor captured disutilities associated with treatment-related adverse events, including severe or serious hypoglycemia, lipohypertrophy, hypoacusis, tonsillar hypertrophy, and tonsillectomy or adenoidectomy.

CADTH was unable to address the following concerns raised from stakeholder input:

- Impacts of the disease and treatment on HRQoL beyond the impact of gains in height (i.e., comorbidities associated with SPIGFD) were not considered.

- No alternative stopping rules were assessed. In the sponsor's base case, patients receiving treatment were assumed to continue on treatment until age 14 for females and 16 for males. CADTH could not alter this assumption.

- Impact of benign or malignant neoplasms on the cost-effectiveness of mecasermin was not captured in the sponsor's submission and could not be addressed by CADTH. The potential impact of this adverse event remains unknown.

\section{Economic Review}

The current review is for mecasermin (Increlex) for growth failure in children and adolescents aged 2 to 18 years with SPIGFD.

\section{Economic Evaluation}

\section{Summary of Sponsor's Economic Evaluation Overview}

The sponsor submitted a cost-utility analysis assessing mecasermin versus no treatment for growth failure in children and adolescents aged 2 to 18 years with SPIGFD. The modelled population was aligned with the Health Canada-indicated population. ${ }^{1}$ 


\section{CADTH}

The recommended dose for mecasermin is $0.04 \mathrm{mg} / \mathrm{kg}$ to $0.12 \mathrm{mg} / \mathrm{kg}$ (40 mcg/ $\mathrm{kg}$ to 120 $\mathrm{mcg} / \mathrm{kg}$ ) administered twice daily by subcutaneous injection. At a submitted price of $\$ 147.92$ per $\mathrm{mg}$ (or $\$ 5,916.64$ per $40 \mathrm{mg}$ vial), the annual cost of mecasermin ranges from $\$ 65,083$ to $\$ 183,416$, based on the recommended dosage, assuming a patient weight of $14.10 \mathrm{~kg}$, and considering wastage of unused product. When considering a patient weight of $35 \mathrm{~kg}$ and the maximum dose, the annual costs of treatment is $\$ 455,581$.

The economic analysis was conducted from the perspective of the Canadian public health care payer over a lifetime time horizon of up to the average life expectancy for females (84.1 years) and males (79.9 years), with the total length of the time horizon depending on a patient's age at treatment initiation. ${ }^{1}$ Costs and clinical outcomes (i.e., QALYs) were discounted at a rate of $1.5 \%$ per annum. ${ }^{1}$ The model was stratified by the patients' age at treatment initiation and sex, and the overall results reflected the results weighted by the distribution of starting ages and sex upon treatment initiation. ${ }^{1}$

\section{Model Structure}

A Markov model structure was developed to capture the long-term costs and outcomes associated with the treatment of SPIGFD. The disease course was modelled via 2 health states: alive and dead. ${ }^{1}$ Each model cycle was 1 year in duration. Patients entered the model alive and on treatment, between the ages of 2 and 5 years, with $60 \%$ of the patient cohort entering at the age of 4 . Patients in the alive state receiving mecasermin were assumed to receive a twice-daily dose of mecasermin subcutaneously. ${ }^{1}$ Patients receiving mecasermin were assumed to remain on treatment unless they discontinued treatment. A stopping rule was implemented such that patients were assumed to discontinue treatment when they reached a maximum age of treatment of 14 years for females or 16 years for males, and this was assumed to coincide with the age at which patients would reach their final height or there would be epiphyseal plate closure. ${ }^{1}$ Patients could remain alive up to a life expectancy of 84.10 years for females and 79.90 years for males, and had a probability of death during each model cycle. Patients who were on treatment had yearly height velocities aligned with the data from the pivotal single-arm trial of mecasermin, whereas patients who discontinued mecasermin or patients receiving no treatment at baseline followed height velocities aligned with natural history data. ${ }^{1}$ Growth was assumed to continue up until 21 years of age. The sponsor's model calculated a patient's height each cycle based on the gain in height via height velocity for that given year, which was then compared with the general population height to determine a patient's height SDS. The height SDS over each model cycle was then used to capture patient utility. ${ }^{1}$

\section{Model Inputs}

The patient cohort comprised pediatric patients with SPIGFD whose baseline characteristics partly reflected characteristics of the patient population from the pivotal trial (Study 1419). ${ }^{2}$ The pivotal trial was used to inform the distribution of patients by sex (43\% female), while key opinion-leader interviews informed the distribution of age of treatment initiation (2 years: 10\%; 3 years: 10\%; 4 years: 10\%; 5 years: 20\%). ${ }^{1}$ Model results were weighted by these 2 distributions. In the model, patient weight at each cycle was used to determine the weightbased dose. For patients who received mecasermin, patient weight at each cycle was derived using the model's predicted height for patients and the general population average body mass index (BMI) for that particular patient age and sex in the general population. For the untreated population, patient weight was derived from patient height in Laron et al. (1993) ${ }^{3}$ and the general population average BMI of the same age and sex. ${ }^{1}$ 
Efficacy data from the pivotal trial, an open-label, multi-centred, investigator-sponsored, single-arm trial ${ }^{2}$ was used to inform yearly height velocities for patients on mecasermin. As the pivotal trial did not include a control arm, the yearly height velocities for untreated patients with SPIGFD were based on growth curves for untreated children with Laron syndrome from infancy to final height, according to their age and sex, as reported in Laron et al. (1993). ${ }^{3}$ Specifically, growth curves of the untreated population reflected untreated hereditary or congenital deficiency of growth hormone-releasing hormone, growth hormone, or insulin-like growth factor $1.1,3$ The annualized height velocity for all years was specific to the treatment year in the model, rather than the age of treatment initiation (i.e., patients starting at age 2 or 3 had the same height velocity in their first year of treatment). ${ }^{1}$ The height velocity in each model cycle was used to determine patient height at the end of the cycle.

The annual probability of treatment discontinuation for patients on mecasermin was obtained from the pivotal trial, which considered factors such as noncompliance, parent or patient decision to withdraw, and poor growth. ${ }^{1}$ The estimate used by the sponsor in its base case was considered a low estimate, with other estimates with higher annual probabilities of discontinuation considered in scenario analyses. The annual probability of treatment-related adverse events with mecasermin included in the model was derived from the proportion of patients who experienced serious adverse events in the pivotal trial. ${ }^{4-6}$

Health-state utility values used in the model were derived from a study that examined the influence of height on HRQoL of life among adults from the general adult population in the UK via the EuroQol 5-Dimensions 3-Levels (EQ-5D-3L) questionnaire. ${ }^{7}$ These values were obtained according to height SDS, for a range of scores from -3.5 to 2.5 . To obtain utility values for patients with a height SDS lower than -3.5 and relevant to the modelled patient population, the sponsor extrapolated utility values for height SDS between -3.5 and -9.5 , based on the relationship between HRQoL and height in Christensen et al. (2007). The sponsor derived 2 extrapolations for utility scores by height SDS, with 1 predicting slightly higher utility scores for height SDS values lower than -3.5 (i.e., an upper limit) based on a linear extrapolation of the relationship between utility and height SDS observed in Christensen et al. (2007), and another predicting utility scores for height SDSs lower than -3.5 (i.e., a lower limit) based on a linear extrapolation, which restricted the data informing the extrapolation to height SDS scores below 0 and had a steeper decline than the upper-limit extrapolation. For its base case, the sponsor derived a third set of extrapolations based on the midpoint of the sponsor's upper- and lower-limit extrapolations. The economic model predicted patient height after each cycle, and then compared that height to the general population height for a patient's given age to derive the height SDS. The sponsor further included disutility values associated with adverse events due to treatment with mecasermin. ${ }^{5}$

Costs captured in the economic model included drug acquisition costs, health care resource utilization and monitoring costs, and adverse event-related costs. ${ }^{18-10}$ The sponsor calculated drug costs based on the price per milligram. In drug cost calculations, the sponsor assumed that patients received $0.224 \mathrm{mg} / \mathrm{kg}$ daily, close to the maximum dose of $120 \mathrm{mcg} / \mathrm{kg}$ twice daily specified by the product monograph, which is aligned with the dosage most patients received in the trial. Drug costs were calculated based on the price per milligram of the product and did not account for potential drug wastage. ${ }^{1}$ 


\section{Summary of Sponsor's Economic Evaluation Results}

The sponsor presented probabilistic analyses for its base case and scenario analyses based on 5,000 iterations. The deterministic results were similar to the probabilistic results. The results presented are weighted by sex and the age of treatment initiation.

\section{Base-Case Results}

In the sponsor's probabilistic base case, mecasermin was more costly (incremental costs: $\$ 2,330,629$ ) and more effective (incremental QALYs: 5.9) than no treatment over the modelled time horizon. The ICER was $\$ 391,879$ per QALY gained. The sponsor's model predicted an average total height gain of $12.5 \mathrm{~cm}$ for mecasermin compared to no treatment.

\section{Sensitivity and Scenario Analysis Results}

The sponsor conducted several sensitivity and scenario analyses. These included exploring the impact of a different distribution for age of treatment initiation; exploring the impact of an equal sex distribution; using a higher assumed BMI relative to the BMI used in the base case (i.e., BMI + 1 standard deviation); shortening the time horizon to 50 years; using Laron (1999) data as an alternative natural history data source for annual height velocity in the untreated population; using the height velocity data from patients in the pivotal trial before their treatment with mecasermin as an alternate natural history data source for growth of the untreated population; applying alternative health-state utility values based on the regression coefficients from Christensen et al. (2007); applying higher estimates for treatment discontinuation based on data from the pivotal trial; and modifying several model parameters (i.e., age distribution, height velocity, daily dose of mecasermin, treatment duration, and adverse event rates) based on data from the EU Registry. ${ }^{1}$ Results remained robust to these changes. ${ }^{1}$

\section{CADTH Appraisal of the Sponsor's Economic Evaluation}

CADTH identified several key limitations to the sponsor's analysis that have notable implications on the economic analysis:

\footnotetext{
- Comparative clinical efficacy and safety of mecasermin compared with no treatment is unknown: The sponsor's pivotal trial was an open-label, multi-centred, investigatorsponsored, single-arm trial that aimed to demonstrate the efficacy of mescasermin. ${ }^{2}$ In the absence of comparative clinical evidence from the pivotal trial to inform the efficacy and safety of no treatment in the submitted model, the sponsor's model was based on a naive comparison to assess the cost-effectiveness of mecasermin compared to no treatment. Data on height and height velocity from the pivotal trial was used to inform the treatment efficacy with mecasermin, and data on height and height velocity from a 1993 natural history study of untreated children with Laron syndrome (i.e., untreated hereditary or congenital deficiency of growth hormone-releasing hormone, growth hormone, or
}

Table 3: Summary of the Sponsor's Economic Evaluation Results

\begin{tabular}{|c|c|c|c|c|c|}
\hline Drug & Total costs (\$) & Incremental costs $(\$)$ & Total QALYs & Incremental QALYs & $\begin{array}{l}\text { ICER vs. no treatment } \\
(\$ \text { per QALY })\end{array}$ \\
\hline No treatment & 19,081 & Reference & 18.3 & Reference & Reference \\
\hline Mecasermin & $2,349,710$ & $2,330,629$ & 24.2 & 5.9 & 391,879 \\
\hline
\end{tabular}

ICER = incremental cost-effectiveness ratio; QALY = quality-adjusted life-year; vs. = versus.

Source: Sponsor's pharmacoeconomic submission. ${ }^{1}$ 
insulin-like growth factor 1) was used to inform growth for patients receiving no treatment. Based on these data, the model predicted a gain in final height of $12.5 \mathrm{~cm}$ between the treated $(136.1 \mathrm{~cm})$ and untreated $(123.6 \mathrm{~cm})$ groups. The sponsor's approach to the incorporation of treatment effects, and the results predicted in the model, are associated with considerable uncertainty. As noted in the clinical review conducted by CADTH, it was not possible to infer a causal relationship between mecasermin treatment and harms outcomes. It does appear that mecasermin may improve final adult height in patients with SPIGFD when compared with historical controls, although the relevance of the historical control group is in question. The clinical expert consulted by CADTH noted that the predicted height gain of $12.5 \mathrm{~cm}$ seemed reasonable but the clinical meaningfulness and translation to HRQoL benefits is uncertain, as discussed in greater detail in the following text. The cost-effectiveness of mecasermin based on the sponsor's submitted model is therefore highly uncertain.

- CADTH was unable to address this limitation. As CADTH's clinical review team was unable to comment on the clinical effectiveness and safety of mecasermin compared with no treatment, all CADTH reanalyses are considered best estimates, rather than base cases, and are considered exploratory in nature.

- There is uncertainty with the impact of height gains on quality of life over a patient's lifetime and generalizability of the utility scores used in the model to patients with SPIGFD: The sponsor's economic model assumed a relationship between HRQoL and height based on a study that assessed EQ-5D-3L utility scores by height SDS. ${ }^{7}$ This study found that mean EQ-5D-3L scores were lower in adults with a greater height deficit (e.g., height SDS $\leq-2$ ) than in taller adults (e.g., height SDS >0), with the EQ-5D-3L score decreasing as patients had an increasingly negative height SDS. Given patients with SPIGFD have a height SDS well below -3.5, the sponsor further extrapolated data from Christensen et al. (2007) to predict the impact of a height SDS below -3.5 on HRQoL, as this was not measured in Christensen et al. (2007). In its base case, the sponsor chose the utility values corresponding to the midpoint between an upper and lower extrapolation, which predicted patients on mecasermin would gain 5.9 QALYs over their lifetime based on a gain in height of $12.5 \mathrm{~cm}$. Several issues with the sponsor's approach to incorporating health-state utility values by height SDS in the submitted model contribute meaningful uncertainty. First, the sponsor's base-case model predicts that patients on mecasermin would stand to benefit what is equivalent to nearly 6 full years of perfect health solely due to a gain of $12.5 \mathrm{~cm}$ in height in childhood, on average. The impact of an approximate $12.5 \mathrm{~cm}$ gain in height from treatment with mecasermin, on average, and whether it would translate to such a gain in HRQoL, particularly in patients well into adulthood with SPIGFD, is highly uncertain. The model does not account for any non-height-related benefits, nor is it possible to correlate gains in height via mecasermin with improvements in comorbidities of SPIGFD. As a result, these predicted QALY gains are solely height-related.

There are also concerns with the generalizability of the relationship between height SDS and utility identified in the general adult population to pediatric patients or adults with SPIGFD, particularly given the fact that people with statures below -3.5 were not included in the original study. The extrapolations used by the sponsor to predict patient utility for patients with SPIGFD are therefore highly uncertain as the assumption of a linear association between height SDS and utility, particularly at a height SDS below -3.5 and in patients with SPIGFD, cannot be confirmed. The sponsor's selection of the midpoint of both the upper and lower extrapolations was arbitrary. In the absence of more appropriate data, the extrapolation based on the entire dataset was likely most appropriate, although another relationship, such as a plateau or threshold effect whereby utility would be the 
same beyond a certain negative height SDS, may exist. Additionally, it is unknown if this relationship would hold over the entirety of a patient's lifetime, or if utilities by height SDS would converge over time. Overall, the approach to incorporating health-state utilities in the model is associated with meaningful uncertainty, as is the resulting magnitude of the benefit predicted by the sponsor's model for mecasermin, along with its cost-effectiveness in comparison with no treatment.

- This key limitation also contributed to CADTH's decision to produce a best estimate rather than a base case due to the significant uncertainty in the impact of a $12.5 \mathrm{~cm}$ gain in height on patient quality of life. In the absence of more appropriate values, CADTH's best-estimate analysis included patient utilities by height SDS based on the sponsor's extrapolated utility values that predicted the least-severe linear decline in utility scores for a height SDS of less than -3.5. In a scenario analysis, CADTH changed the time horizon to 21 years (the maximum treatment length) to explore the impact of the assumption of the benefit of treatment in childhood until the age at which patients were assumed to stop growing on QALY gains attributed to mecasermin. CADTH also tested a scenario in which the utility scores declined 0.01 for each 0.5 change in height SDS below -3.5.

- The generalizability of the results of the pivotal trial to all Canadian patients with SPIGFD is uncertain: The pivotal trial included an international group of patients with genetically proven SPIGFD. The clinical expert consulted by CADTH for this review indicated that it is likely that the findings of the pivotal trial may be generalizable to Canadian patients with genetic causes of SPIGFD over the age of 18 months with a height SDS of less than -3; however, uncertainty remains with regard to the treatment effect of mecasermin in patients without a genetic cause of SPIGFD who may have been excluded from the study.

- CADTH was unable to address this limitation.

- Sex distribution of the modelled patient population is not aligned with expectations of the Canadian population with SPIGFD: The sponsor's economic model assumed that $57 \%$ of the indicated population was male, based on the sex distribution of the pivotal trial. ${ }^{2}$ The clinical expert consulted by CADTH indicated that, despite the sex distribution reported in the pivotal trial, the prevalence of SPIGFD is equal between males and females. The ICERs in females were higher for those in males, and, given that the results are weighted by sex in addition to age of treatment initiation, this likely biased results in favour of mecasermin.

- CADTH adjusted the sex distribution to be equal for males and females in the CADTH best estimate.

- Drug costs are likely underestimated due to exclusion of wastage: The sponsor's economic model calculated drug costs by deriving the cost per milligram (i.e., the price of a $40 \mathrm{mg} / 4 \mathrm{~mL}$ vial divided by the number of milligrams of drug per vial) despite the fact that mecasermin is dispensed as full $40 \mathrm{mg}$ vials. As the $40 \mathrm{mg}$ vial is the smallest dispensable unit, the whole vial dispensed would be paid for by drug plans. Costs associated with mecasermin were likely underestimated as the sponsor's calculations did not account for drug costs attributed to potential drug wastage of unused product. Such costs could have been captured by estimating the number of vials to cover the required dose in a given year rather than the exact milligrams.

- CADTH addressed this limitation by adjusting drug cost calculations to account for drug wastage by considering the number of vials required to cover a patient per yearly cycle.

Key assumptions made by the sponsor and appraised by CADTH are listed in Table 4. 


\section{Table 4: Key Assumptions of the Submitted Economic Evaluation (Not Noted as Limitations to the Submission)}

Sponsor's key assumption

The time horizon was based on the life expectancy of individuals with SPGFD and is similar to the general population of the same age and sex (males $=79.9$ and females $=84.1$ ).

Age distribution for age of treatment initiation in model is reflective of Canadian patients with SPIGFD (i.e., 2 years $=$ $10 \% ; 3$ years $=10 \% ; 4$ years $=60 \% ; 5$ years $=20 \%$ )
CADTH comment

Appropriate

Appropriate. The clinical expert consulted by CADTH indicated that the distribution of age of treatment initiation (with all patients treated from age 2 to 5 years) appears reasonable and is not expected to differ between females and males in Canada. There is misalignment between the age of patients at baseline in the pivotal trial and the age of patients anticipated to initiate treatment in Canada. CADTH conducted a scenario analysis to assess the impact of assuming an older age of treatment initiation on the cost-effectiveness of mecasermin.

The natural history of untreated individuals with SPIGFD is aligned with the data from a study by Laron et al. (1993), which assessed yearly height velocity in patients with Laron syndrome.

Uncertain. The height and height velocity data in Laron et al. (1993) align with expected height velocities of untreated patients with SPIGFD in Canadian clinical practice. However, the clinical expert consulted by CADTH speculated that, due to changes in the natural environment and improved nutrition, it is possible that, since 1993, patients with Laron syndrome may have improved height velocities compared with this data; however, this has not been studied.

The pattern of height velocity by year of treatment remains unchanged regardless of the age of treatment initiation.

Appropriate. The clinical expert consulted by CADTH indicated that it is expected that the pattern of height velocity would remain unchanged regardless of patient age of treatment initiation. For example, if a patient had initiated treatment at age 6 , it is reasonable to expect a height velocity in the first year similar to that experienced by a patient who started treatment at age 3 .

Likely appropriate in the absence of data specific to patients with SPIGFD. calculate the weight of pediatric patients who received mecasermin in the economic model, to estimate the weightbased dose and therefore drug costs.

In the economic model, serious adverse events associated with mecasermin were derived from the pivotal trial. Serious adverse events included severe or serious hypoglycemia, lipohypertrophy, hypoacusis, tonsillar hypertrophy, tonsillectomy or adenoidectomy, and intracranial hypertension.

A dosage of $0.224 \mathrm{mg} / \mathrm{kg}$ per day of mecasermin is assumed to be administered in clinical practice.
Likely appropriate, according to the clinical expert consulted by CADTH. However, given the single-arm nature of the pivotal trial, it is difficult to determine the comparative safety of mecasermin with no treatment.

Likely appropriate, according to the clinical expert consulted by CADTH. CADTH conducted a scenario analysis assessing the impact of assuming a maximal dose of $0.24 \mathrm{mg} / \mathrm{kg}$ per day. 
Twice-daily injections with mecasermin is not associated with a disutility.
Uncertain. Studies in other indications have identified disutilities associated with the frequency of injectable treatments. There is uncertainty with the magnitude of this potential disutility in pediatric patients with SPIGFD. If there is an impact of twicedaily injections on patient quality of life, then the total QALYS associated with mecasermin predicted in the sponsor's model are likely overestimated.

BMI = body mass index; $\mathrm{QALY}=$ quality-adjusted life-year; SPIGFD = severe primary insulin-like growth factor 1 deficiency.

\section{CADTH Reanalyses of the Economic Evaluation}

\section{Exploratory Analysis Results}

The CADTH best estimate was derived by making changes in model parameter values and assumptions, in consultation with a clinical expert. CADTH undertook the reanalyses outlined in Table 5 to address, where possible, the limitations with the sponsor's submitted economic model. CADTH was unable to address important limitations related to the comparative efficacy and safety of mecasermin in comparison with no treatment; the impact of a $12.5 \mathrm{~cm}$ gain in height on patient HRQoL and uncertainty in the applicability of utility weights by height SDS from the general population to patients with SPIGFD; and the generalizability of the results to all patients covered under the Health Canada indication.

Results for the stepwise analyses undertaken for the CADTH best-estimate analysis are found in Table 6. The change to the health-state utilities that predicted a less-severe decline in utility scores as height SDS decreased in comparison with the sponsor's approach had the greatest impact of the stepped changes. The probabilistic CADTH best-estimate analysis found that mecasermin was associated with incremental costs of $\$ 2,338,189$ and an incremental benefit of 3.8 QALYs compared with no treatment over the lifetime time horizon. The ICER,

\section{Table 5: CADTH Revisions to the Submitted Economic Evaluation}

\begin{tabular}{|c|c|c|}
\hline Stepped analysis & Sponsor's value or assumption & CADTH value or assumption \\
\hline \multicolumn{3}{|c|}{ Corrections to sponsor's base case } \\
\hline None & None & None \\
\hline \multicolumn{3}{|c|}{ Changes to derive the CADTH best-estimate analysis ${ }^{a}$} \\
\hline 1. Health-state utilities & $\begin{array}{l}\text { Utility values derived from the midpoint } \\
\text { between } 2 \text { extrapolations (the upper limit and } \\
\text { the lower limit) of the relationship between } \\
\text { height SDS and utility estimated by the } \\
\text { sponsor for height SDS below }-3.5\end{array}$ & $\begin{array}{l}\text { Utility values based on the upper limit of the } \\
\text { sponsor's extrapolation of the relationship } \\
\text { between height SDS and utility (i.e., least- } \\
\text { severe decline in patient utility as height } \\
\text { SDS declines below -3.5) }\end{array}$ \\
\hline $\begin{array}{l}\text { 2. Proportion of males and } \\
\text { females }\end{array}$ & $\begin{array}{l}\text { Males: } 57 \% \\
\text { Females: } 43 \%\end{array}$ & Equal sex distribution \\
\hline 3. Adjusting drug cost calculations & $\begin{array}{l}\text { Calculated based on cost per milligram and } \\
\text { exact number of milligrams to cover yearly } \\
\text { dose }\end{array}$ & $\begin{array}{l}\text { Calculated based on cost per vial and the } \\
\text { exact number of full vials needed to cover } \\
\text { yearly dose }\end{array}$ \\
\hline CADTH best-estimate analysis & \multicolumn{2}{|l|}{ Reanalyses $1+2+3$} \\
\hline
\end{tabular}

SDS = standard deviation score

aln the absence of a valid comparator, CADTH has provided a best-estimate analysis of the cost-effectiveness of mecasermin compared to no treatment. 
weighted by age of treatment initiation and sex, for mecasermin versus no treatment was $\$ 624,249$ per QALY gained. Mecasermin had a 0\% probability of being cost-effective at a willingness-to-pay threshold of $\$ 50,000$ per QALY gained and $99 \%$ of the incremental costs were from drug acquisition costs. When considering the various ages of patients assumed to initiate treatment in the CADTH best estimate (2 to 5 years), the ICER ranged from $\$ 603,599$ to $\$ 704,562$ per QALY gained.

\section{Scenario Analysis Results}

CADTH undertook a series of price-reduction analyses on the price of mecasermin based on the sponsor's base case and the CADTH best estimate (Table 7). In the CADTH best-estimate analysis, mecasermin may be cost-effective at a willingness-to-pay threshold of $\$ 50,000$ per QALY with a price reduction of at least $92 \%$.

CADTH also undertook several scenario analyses to determine the impact of alternative assumptions on the cost-effectiveness of mecasermin versus no treatment, which included:

- shortening the time horizon to 21 years

- exploring a decline in utility of 0.01 for each -0.5 step in height SDS for all height SDSs below -3.5

- assuming an alternate distribution for the age of treatment initiation which was older than that in the CADTH base case ( 5 years: $25 \%$; 6 years: $25 \%$; 7 years: $25 \%$; 8 years: $25 \%$ ); this was arbitrarily chosen to highlight the impact of age of treatment initiation on model results

- assuming a daily dose of $0.24 \mathrm{mg} / \mathrm{kg}$ for mecasermin, which is aligned with the maximum recommended dose in the product monograph.

The results of these analyses are presented in Appendix 4, Table 12. Results were most sensitive to the shortened time horizon of 21 years (ICER $=1,594,377$ per QALY gained) and the use of a 0.01 decline in utility for each -0.5 step in height SDS below and height SDS of -3.5 (ICER $=1,593,918$ per QALY gained), which highlights the impact a reduction

Table 6: Summary of the Stepped Analysis of the CADTH Reanalysis Results

\begin{tabular}{|c|c|c|c|c|}
\hline Stepped analysis & Drug & Total costs $(\$)$ & Total QALYs & ICER (\$ per QALY) \\
\hline \multirow[t]{2}{*}{ Sponsor's base case } & No treatment ${ }^{\mathrm{a}}$ & 19,081 & 18.3 & Reference \\
\hline & Mecasermin & $2,349,710$ & 24.2 & 391,879 \\
\hline \multirow[t]{2}{*}{ CADTH reanalysis 1} & No treatment ${ }^{a}$ & 19,125 & 23.3 & Reference \\
\hline & Mecasermin & $2,396,528$ & 27.1 & 609,674 \\
\hline \multirow[t]{2}{*}{ CADTH reanalysis 2} & No treatment ${ }^{a}$ & 19,240 & 18.6 & Reference \\
\hline & Mecasermin & $2,356,080$ & 24.3 & 407,260 \\
\hline \multirow[t]{2}{*}{ CADTH reanalysis 3} & No treatment ${ }^{\mathrm{a}}$ & 19,115 & 18.2 & Reference \\
\hline & Mecasermin & $2,392,267$ & 24.2 & 399,895 \\
\hline \multirow[t]{2}{*}{ CADTH best estimate } & No treatment ${ }^{\mathrm{a}}$ & 19,274 & 23.3 & Reference \\
\hline & Mecasermin & $2,357,463$ & 27.1 & 624,249 \\
\hline
\end{tabular}

ICER = incremental cost-effectiveness ratio; QALY = quality-adjusted life-year. aReference product is the least costly alternative. 
in the magnitude of benefit on HRQoL from a gain in height with mecasermin would have on its cost-effectiveness. Based on comparing the CADTH best estimate with the scenario assessing a time horizon of 21 years, approximately $38 \%$ of the incremental QALYs gained with mecasermin in the CADTH best estimate are from the period during which patients are expected to be growing, with the remaining $62 \%$ from the period when patients are at their final adult height.

\section{Issues for Consideration}

Use of leuprolide - The clinical expert consulted by CADTH noted that, although leuprolide is not approved for the given indication in Canada, some clinicians may choose to prescribe leuprolide in conjunction with mecasermin for patients with SPIGFD to prolong the growth period. The decision to prescribe leuprolide would be made on a case-by-case basis, and in Canadian clinical practice it is likely reserved for patients who are near to their final adult height or for whom bone age is rapidly advancing. The cost-effectiveness of mecasermin in situations where leuprolide is used is unknown.

\section{Overall Conclusions}

The clinical evidence submitted by the sponsor demonstrated that treatment with mecasermin may improve final adult height in pediatric patients with SPIGFD compared with untreated patients with Laron syndrome. Due to the lack of a randomized control group in the pivotal trial, it was not possible to infer a causal relationship between mecasermin treatment and growth and harms outcomes and as such the comparative clinical efficacy and safety of mecasermin compared to no treatment remains unknown.

CADTH identified several major limitations with the submitted economic evaluation beyond those related to the clinical evidence. These included issues related to the utility values by

\section{Table 7: CADTH Price-Reduction Analyses}

\begin{tabular}{|l|c|c|}
\hline Analysis & \multicolumn{2}{|c|}{ ICERs for mecasermin vs. no treatment (\$ per QALY) } \\
\hline Price reduction & Sponsor base case & CADTH reanalysis \\
\hline No price reduction & 391,879 & 624,249 \\
\hline $10 \%$ & 349,063 & 548,043 \\
\hline $20 \%$ & 307,937 & 482,537 \\
\hline $30 \%$ & 274,447 & 436,154 \\
\hline $40 \%$ & 236,843 & 369,270 \\
\hline $50 \%$ & 193,032 & 310,077 \\
\hline $60 \%$ & 152,893 & 249,296 \\
\hline $70 \%$ & 116,790 & 186,471 \\
\hline $80 \%$ & 78,581 & 124,636 \\
\hline $90 \%$ & 39,473 & 62,136 \\
\hline $91 \%$ & 35,245 & 56,448 \\
\hline $92 \%$ & 30,968 & 49,579 \\
\hline
\end{tabular}

ICER = incremental cost-effectiveness ratio; QALY = quality-adjusted life-year; vs. = versus. 


\section{CADTH}

height SDS applied in the model and the impact of a gain of $12.5 \mathrm{~cm}$ in height, on average, on HRQoL benefit over a patient's lifetime; generalizability of the results to all Canadian patients under the Health Canada indication; the proportion of males and females with SPIGFD in the Canadian setting; and the lack of consideration of potential drug wastage.

Due to the lack of available comparative clinical efficacy and safety data, as well as uncertainty in the impact of a $12.5 \mathrm{~cm}$ gain in height on patient quality of life over their lifetime, the CADTH reanalysis consisted of a best estimate rather than a base case. The best-estimate reanalysis included revising health-state utilities to reflect a less-severe decline in utility scores for height SDS less than -3.5 in the absence of available data; assuming an equal proportion of males and females to better align with the distribution in Canadian clinical practice; and adjusting the calculation of drug costs to account for wastage. The CADTH bestestimate results were aligned with the results reported by the sponsor, as mecasermin was not cost-effective at conventional willingness-to-pay thresholds. In the CADTH best estimate, mecasermin was associated with an ICER of $\$ 624,249$ per QALY gained (incremental costs of $\$ 2,338,189$ and an incremental benefit of 3.8 QALYS) compared with no treatment. A price reduction of at least $92 \%$ would be required for mecasermin to be considered cost-effective at a willingness-to-pay threshold of $\$ 50,000$ per QALY gained.

At the sponsor-submitted price, mecasermin is not cost-effective and uncertainty remains with regard to the magnitude of benefit associated with the gains in height predicted by the sponsor's model. The economic model predicted a gain in final height of $11.8 \mathrm{~cm}$ for patients treated with mecasermin compared with no treatment, which led to a gain of 3.8 QALYS, or nearly 4 additional years of perfect health over their lifetime in the CADTH best estimate. Given the uncertainty in both the clinical evidence informing the gain in height, as well as the impact of gains in height on patient utility scores, these QALY gains are highly uncertain and the ICER for mecasermin in comparison with no treatment is potentially underestimated. While there is uncertainty regarding the clinical benefits of mecasermin, the drug acquisition costs of mecasermin over the patient's lifetime $(\$ 2,335,609$ incremental drug costs as predicted by the model) are not associated with the same level of uncertainty. 


\section{References}

1. Pharmacoeconomic evaluation [internal sponsor's report]. In: Drug Reimbursement Review sponsor submission: Increlex (mecasermin), 10mg/mL (40mg per vial) subcutaneous injection. Mississauga (ON): Ipsen Biopharmaceuticals Canada, Inc; 2021 Jun 14.

2. Clinical Study Report: study 1419. A study of long-term recombinant human insulin-like growth factor (rh/GF-1) treatment of children with short stature due to severe primary IGF-1 deficiency study [internal sponsor's report]. Basking Ridge (NJ): Ipsen Biopharmaceuticals Inc; 2012.

3. Laron Z, Lilos P, Klinger B. Growth curves for Laron syndrome. Arch Dis Child. 1993;68(6):768-770. PubMed

4. Pollock RF, Heller S, Pieber TR, et al. Short-term cost-utility of degludec versus glargine U100 for patients with type 2 diabetes at high risk of hypoglycaemia and cardiovascular events: a Canadian setting (DEVOTE 9). Diabetes Obes Metab. 2019;21(7):1706-1714. PubMed

5. Sullivan PW, Ghushchyan V. Preference-based EQ-5D index scores for chronic conditions in the United States. Med Decis Making. 2006;26(4):410-420. PubMed

6. Harris S, Mamdani M, Galbo-Jørgensen CB, Bøgelund M, Gundgaard J, Groleau D. The effect of hypoglycemia on health-related quality of life: Canadian results from a multinational time trade-off survey. Can J Diabetes. 2014;38(1):45-52. PubMed

7. Christensen T, Djurhuus C, Clayton P, Christiansen J. An evaluation of the relationship between adult height and health-related quality of life in the general UK population. Clin Endocrinol (Oxf). 2007;67:407-412. PubMed

8. Canadian Institute of Health Information (CIHI). Your health system. Profile: Ontario. 2020; https://yourhealthsystem.cihi.ca/hsp/inbrief;jsessionid=XtwozHh6rK -sOFdGoliSWf8K.yhs?lang=en\#!/search/2/C5001. Accessed 2021 Sep 2.

9. Guidance document for the costing of health care resources in the Canadian setting. Ottawa (ON): CADTH; 2017: https://www.cadth.ca/guidance-document-costing -health-care-resources-canadian-setting. Accessed 2020 Sep 2.

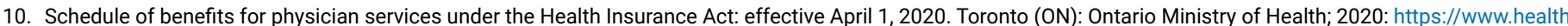
.gov.on.ca/en/pro/programs/ohip/sob/physserv/sob_master20200306.pdf. Accessed 2020 Sep 2.

11. Budget Impact Analysis [internal sponsor's report]. In: Drug Reimbursement Review sponsor submission: Increlex (mecasermin), 10mg/mL (40mg per vial) subcutaneous injection. Mississauga (ON): Ipsen Biopharmaceuticals Canada, Inc; 2021 Jun 14.

12. Statistics Canada. Table 17-10-0016-01. Estimates of births, by sex, annual. 2021: 10.25318/1710001601-eng. Accessed 2020 Sep 2 . 


\section{Appendix 1: Cost Comparison Table}

Note that this appendix has not been copy-edited.

The comparators presented in the following table have been deemed to be appropriate based on feedback from clinical expert(s). Comparators may be recommended (appropriate) practice or actual practice. Existing product listing agreements are not reflected in the table and as such, the table may not represent the actual costs to public drug plans.

Table 8: CADTH Cost Comparison Table for Growth Failure in Children and Adolescents With Confirmed Severe Primary Insulin-Like Growth Factor 1 Deficiency

\begin{tabular}{|c|c|c|c|c|c|c|}
\hline Treatment & Strength & Form & Price & $\begin{array}{l}\text { Recommended } \\
\text { dosage }\end{array}$ & Daily cost (\$) & $\begin{array}{l}\text { Course or annual } \\
\text { cost }^{\mathrm{a}}\end{array}$ \\
\hline $\begin{array}{l}\text { Mecasermin } \\
\text { (Increlex) }\end{array}$ & $10 \mathrm{mg} / \mathrm{mL}$ & $\begin{array}{l}\text { Vial for } \\
\text { subcutaneous } \\
\text { injection } \\
4 \mathrm{~mL}\end{array}$ & $5,916.6400$ & $\begin{array}{l}0.04 \text { to } 0.08 \mathrm{mg} / \\
\mathrm{kg} \text { twice daily for } \\
\text { first week. If well } \\
\text { tolerated, dose } \\
\text { may be increased } \\
\text { to maximum of } \\
0.12 \mathrm{mg} / \mathrm{kg} \text { twice } \\
\text { daily. }\end{array}$ & 178.31 to 502.51 & $\begin{array}{c}65,083 \text { to } \\
183,416\end{array}$ \\
\hline
\end{tabular}

Note: Weight-based doses use an average weight of $14.10 \mathrm{~kg}$. Assumes daily doses and wastage. Annual costs calculated based on 365 days per year. aSponsor's submitted price. ${ }^{1}$ 


\section{Appendix 2: Submission Quality}

Note that this appendix has not been copy-edited.

\section{Table 9: Submission Quality}

\begin{tabular}{|l|c|l|}
\hline Description & Yes/No & No \\
\hline $\begin{array}{l}\text { Population is relevant, with no critical intervention missing, } \\
\text { and no relevant outcome missing }\end{array}$ & Yes & No comment \\
\hline $\begin{array}{l}\text { Model has been adequately programmed and has } \\
\text { sufficient face validity }\end{array}$ & Yes & No comment \\
\hline Model structure is adequate for decision problem & Yes comment \\
\hline $\begin{array}{l}\text { Data incorporation into the model has been done } \\
\text { adequately (e.g., parameters for probabilistic analysis) }\end{array}$ & Yes & No comment \\
\hline $\begin{array}{l}\text { Parameter and structural uncertainty were adequately } \\
\text { assessed; analyses were adequate to inform the decision } \\
\text { problem }\end{array}$ & & No comment \\
\hline $\begin{array}{l}\text { The submission was well organized and complete; the } \\
\text { information was easy to locate (clear and transparent } \\
\text { reporting; technical documentation available in enough } \\
\text { details) }\end{array}$ & Yes & \\
\hline
\end{tabular}

Note: This table has not been copy-edited. 


\section{Appendix 3: Additional Information on the Submitted Economic Evaluation}

Note that this appendix has not been copy-edited.

There is no additional information from the sponsor's submitted pharmacoeconomic evaluation to report. 


\section{Appendix 4: Additional Details on the CADTH Reanalyses and Sensitivity Analyses of the Economic Evaluation}

Note that this appendix has not been copy-edited.

Detailed Results of CADTH Base Case

Table 10: Disaggregated Summary of CADTH's Economic Evaluation Results

\begin{tabular}{|c|c|c|c|}
\hline Treatment & Component & Value & Incremental (versus no treatment) \\
\hline \multicolumn{4}{|c|}{ Discounted QALYs } \\
\hline No treatment & Total & 23.5 & NA \\
\hline Mecasermin & Total & 27.2 & 3.8 \\
\hline \multicolumn{4}{|c|}{ Discounted costs $(\$)^{\mathrm{a}}$} \\
\hline \multirow[t]{4}{*}{ No treatment } & Drug acquisition & 0 & NA \\
\hline & Adverse events & 0 & NA \\
\hline & Management costs & 19,274 & NA \\
\hline & Total & 19,274 & NA \\
\hline \multirow[t]{4}{*}{ Mecasermin } & Drug acquisition & $2,335,609$ & $2,335,609$ \\
\hline & Adverse events & 381 & 381 \\
\hline & Management costs & 21,473 & 2,198 \\
\hline & Total & $2,357,463$ & $2,338,189$ \\
\hline \multicolumn{4}{|c|}{ ICER vs. reference ( $\$$ per QALY) } \\
\hline No treatment & & \multicolumn{2}{|c|}{ Reference } \\
\hline Mecasermin & & \multicolumn{2}{|r|}{624,249} \\
\hline
\end{tabular}

ICER = incremental cost-effectiveness ratio; NA = not applicable; QALY = quality-adjusted life-year; vs. = versus.

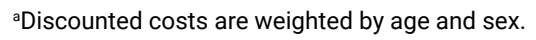

Table 11: Disaggregated Summary of CADTH's Economic Evaluation Results Weighted by Proportion of Patients by Age of Treatment Initiation

\begin{tabular}{|c|c|c|c|c|}
\hline Parameter & $\begin{array}{l}\text { Proportion of patients by age of } \\
\text { treatment initiation }\end{array}$ & Mecasermin & $\begin{array}{l}\text { No treatment } \\
\text { (reference) }\end{array}$ & Incremental \\
\hline \multicolumn{5}{|c|}{ Discounted QALYs } \\
\hline Total $^{\mathrm{a}}$ & NA & 27.2 & 23.5 & 3.6 \\
\hline Age 2 & $10 \%$ & 27.2 & 23.7 & 3.5 \\
\hline Age 3 & $10 \%$ & 27.4 & 23.6 & 3.9 \\
\hline Age 4 & $60 \%$ & 27.2 & 23.4 & 3.8 \\
\hline Age 5 & $20 \%$ & 27.0 & 23.1 & 3.7 \\
\hline
\end{tabular}




\begin{tabular}{|c|c|c|c|c|}
\hline Parameter & $\begin{array}{l}\text { Proportion of patients by age of } \\
\text { treatment initiation }\end{array}$ & Mecasermin & $\begin{array}{l}\text { No treatment } \\
\text { (reference) }\end{array}$ & Incremental \\
\hline \multicolumn{5}{|c|}{ Discounted costs $(\$)$} \\
\hline Totala $^{\mathrm{a}}$ & NA & $2,338,189$ & 19,274 & $2,338,189$ \\
\hline Age 2 & $10 \%$ & $2,463,720$ & 19,548 & $\$ 2,463,720$ \\
\hline Age 3 & $10 \%$ & $2,426,355$ & 19,406 & $\$ 2,426,355$ \\
\hline Age 4 & $60 \%$ & $2,333,824$ & 19,261 & $\$ 2,333,824$ \\
\hline Age 5 & $20 \%$ & $2,244,434$ & 19,113 & $\$ 2,244,434$ \\
\hline ICER (\$ per QALY) & \multicolumn{4}{|c|}{$624,249^{\mathrm{b}}(603,599$ to 704,562$)$} \\
\hline
\end{tabular}

ICER = incremental cost-effectiveness ratio; QALY = quality-adjusted life-year.

aTotal weighted by proportion of patients within age distribution, as well as by sex.

${ }^{\mathrm{b} C A D T H ' s ~ p r o b a b i l i s t i c ~ b a s e ~ c a s e . ~}$

\section{Detailed Results of CADTH Base Case}

Scenario Analyses

Table 12: Scenario Analyses for Mecasermin vs. No Treatment

\begin{tabular}{|c|c|c|c|c|}
\hline Scenario analysis & Drug & Total costs (\$) & Total QALYs & ICER (\$ per QALY) \\
\hline \multirow[t]{2}{*}{ CADTH base case } & No treatment ${ }^{a}$ & 19,274 & 23.31 & Reference \\
\hline & Mecasermin & $2,357,463$ & 27.12 & 624,249 \\
\hline \multirow[t]{2}{*}{ Time horizon $=21$ years } & No treatment ${ }^{a}$ & 7,870 & 9.68 & Reference \\
\hline & Mecasermin & $2,348,544$ & 11.15 & $1,593,880$ \\
\hline \multirow{2}{*}{$\begin{array}{l}\text { Utility decline of } 0.01 \text { for all } \\
\text { height SDSs below }-3.5\end{array}$} & No treatment ${ }^{a}$ & 19,120 & 29.25 & Reference \\
\hline & Mecasermin & $2,356,645$ & 30.72 & $1,593,918$ \\
\hline \multirow[t]{2}{*}{ Alternate age distribution } & No treatment ${ }^{a}$ & 18,998 & 23.15 & Reference \\
\hline & Mecasermin & $2,063,437$ & 26.64 & 585,544 \\
\hline \multirow{2}{*}{$\begin{array}{l}\text { Daily dose of } 0.24 \mathrm{mg} / \mathrm{kg} \text { for } \\
\text { mecasermin }\end{array}$} & No treatment ${ }^{a}$ & 19,150 & 23.46 & Reference \\
\hline & Mecasermin & $2,353,490$ & 27.12 & 637,709 \\
\hline
\end{tabular}

SDS = standard deviation; vs. = versus.

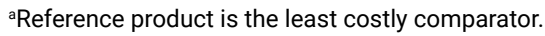




\section{Appendix 5: Submitted Budget Impact Analysis and CADTH Appraisal}

Note that this appendix has not been copy-edited.

\section{Table 13: Summary of Key Take-Aways}

Key take-aways of the budget impact analysis

- CADTH identified the following key limitations with the sponsor's analysis:

o Drug costs may be underestimated due to the exclusion of potential drug wastage.

oThere is uncertainty around the estimates used to determine the size of the population eligible for treatment with mecasermin (i.e., incidence, prevalence, and diagnosis rate).

- CADTH undertook a reanalysis to derive the CADTH base case by only adjusting the drug costs of mecasermin based on the cost per vials needed to cover a yearly dose, rather than the exact cost per milligram, which had a limited impact on results. The estimated budget impact with the reimbursement of mecasermin was $\$ 11,572,125$ in year $1, \$ 12,035,169$ in year 2 , and $\$ 12,374,828$ in year 3, for a 3-year budget impact of $\$ 35,982,122$. However, there remains some uncertainty with the sponsor's estimated budget impact due to uncertainty in the potential population size.

\section{Summary of Sponsor's Budget Impact Analysis}

The sponsor assessed the budget impact of the introduction of mecasermin for patients with SPIGFD, from the perspective of the public drug plans in Canada (excluding Quebec), over a 3-year time horizon. ${ }^{11}$ The sponsor included drug acquisition costs and a pharmacy mark-up. In the reference scenario, the sponsor assumed that patients do not receive any active treatment for SPIGFD and thus, no costs are incurred by public drug plans. In the new drug scenario, mecasermin was assumed to capture the entire market. Drug costs of mecasermin were calculated based on patient weight. ${ }^{11}$

The sponsor used an epidemiological approach to identify the total population eligible for treatment with mecasermin, which included identifying the prevalent and incident number of cases of SPIGFD over the age range which patients are expected to receive treatment (2 to 18 years)..$^{11}$ Specifically, the prevalent population represented children with SPIGFD born prior to 2019 who would be considered for treatment, and the incident population represented children with SPIGFD born in 2019 who would be 2 years of age in 2021 (baseline year) and likely to be newly diagnosed. The sponsor further restricted the population to the proportion of patients with SPIGFD actually diagnosed with SPIGFD. As drug costs are weight dependent, patient weight was derived using the BMI of the general population of the same age and sex, and age- and -sex specific height estimates from Laron et al. (1993). ${ }^{3}$

The sponsor's budget impact analysis (BIA) also included the following key assumptions:

- The incidence of SPIGFD was assumed to remain constant over time at $0.0016 \%{ }^{12}$

- The diagnosis rate was assumed to be the same prior to and after the introduction of mecasermin.

- The age of treatment initiation for males and females was assumed to be 2 years of age. Males were assumed to be treated up to age 16 and females were assumed to be treated up to age 14.

- All eligible patients (100\%) are reimbursed by Canadian publicly funded drug plans.

Key inputs to the BIA are documented in Table 14. 
Table 14: Summary of Key Model Parameters

\begin{tabular}{|c|c|}
\hline Parameter & $\begin{array}{l}\text { Sponsor's estimate } \\
\text { (reported as year } 1 / \text { year } 2 / \text { year } 3 \text { if appropriate) }\end{array}$ \\
\hline \multicolumn{2}{|c|}{ Target population } \\
\hline Proportion of patients diagnosed with SPIGFD & $78 \%$ \\
\hline Incidence of SPIGFD in children & $0.0016 \%$ \\
\hline Number of patients eligible for drug under review & $54 / 54 / 54$ \\
\hline \multicolumn{2}{|c|}{ Market uptake (3 years) } \\
\hline $\begin{array}{l}\text { Uptake (reference scenario) } \\
\text { No treatment }\end{array}$ & $100 \% / 100 \% / 100 \%$ \\
\hline $\begin{array}{l}\text { Uptake (new drug scenario) } \\
\text { Mecasermin } \\
\text { No active treatment }\end{array}$ & $\begin{array}{c}100 \% / 100 \% / 100 \% \\
0 \% / 0 \% / 0 \%\end{array}$ \\
\hline \multicolumn{2}{|c|}{ Cost of treatment } \\
\hline $\begin{array}{l}\text { Cost of treatment } \\
\text { Mecasermin - per vial } \\
\text { No active treatment }\end{array}$ & $\begin{array}{l}\$ 5,916.64 \\
\$ 0\end{array}$ \\
\hline
\end{tabular}

SPIGFD = severe primary insulin-like growth factor 1 .

Note: Cost of treatment per patient is dose-dependent and varies by age. The assumed dose for all drug cost calculations was $0.224 \mathrm{mg} / \mathrm{kg}$.

${ }^{\text {aC }}$ ost of treatment per patient per year varies with patient weight.

Source: Sponsor's submitted budget impact analysis report. ${ }^{11}$

\section{Summary of the Sponsor's Budget Impact Analysis Results}

Results of the sponsor's base-case analysis suggest that the introduction of mecasermin in patients with SPIGFD would result in an incremental budget impact of $\$ 11,417,373$ in Year 1, $\$ 11,867,717$ in Year 2, and $\$ 12,201,390$ in Year 3, for a total incremental budget impact of $\$ 35,486,480$ over the 3-year time horizon. ${ }^{11}$

\section{CADTH Appraisal of the Sponsor's Budget Impact Analysis}

CADTH identified several key limitations to the sponsor's analysis that have notable implications on the results of the BIA:

- Drug costs are likely underestimated due to exclusion of wastage: In the sponsor's submitted pharmacoeconomic and budget impact analyses, the sponsor calculated drug costs by deriving the cost per $\mathrm{mg}$ (i.e., price of a $40 \mathrm{mg} / 4 \mathrm{~mL}$ vial divided by the number of milligrams of drug per vial) despite the fact that mecasermin is dispensed as full $40 \mathrm{mg}$ vials. As such, the sponsor's calculations of drug costs associated with mecasermin were underestimated (See CADTH Appraisal of the Sponsor's Economic Evaluation). To align the BIA with CADTH's best estimate analysis of the submitted pharmacoeconomic review, drug cost calculations were adjusted to consider the drug wastage from the number of full vials of product required to cover the dose in a given year rather than the exact milligrams.

- CADTH addressed this limitation by adjusting drug cost calculations to account for drug wastage by considering the number of vials required to cover a patient per yearly cycle.

- The estimated eligible population size is uncertain due to the uncertainty in several epidemiological inputs: In the sponsor's submitted BIA, the population eligible for mecasermin was estimated based in part on the estimated prevalence of SPIGFD in Canada (excluding Quebec) and the estimated proportion of patients with SPIGFD actually diagnosed with SPIGFD (78\%) in practice. The clinical expert consulted by CADTH indicated that the prevalence of SPIGFD in the sponsor's BIA is likely based on the most stringent 
definition of SPIGFD (i.e., true Laron dwarfism), which could be confirmed by genetic or lab testing if such a test were available, and as such, all patients meeting the criteria of true Laron dwarfism would be diagnosed. Additionally, the sponsor estimated the total incidence of SPIGFD (considering undiagnosed and diagnosed patients) was $0.0016 \%$. The clinical expert consulted by CADTH indicated that while the sponsor's estimate appears to be reasonable, the incidence of patients with SPIGFD in Canada is unknown and highly uncertain in the absence of any published Canadian literature.

- CADTH did not address this limitation in the absence of more appropriate inputs. In a scenario analysis, CADTH explored the uncertainty in the estimated eligible population size by assuming that (a) all patients in the model with SPIGFD are diagnosed and seek treatment; and (b) arbitrarily changing the incidence rate of SPIGFD to reflect a $25 \%$ and $50 \%$ increase, respectively.

\section{CADTH Reanalyses of the Budget Impact Analysis}

A table noting the change made to the sponsor's BIA as part of the CADTH reanalysis is available in Table 15. All analyses are from the public drug plan perspective, unless otherwise noted.

\section{Table 15: CADTH Revisions to the Submitted Budget Impact Analysis}

\begin{tabular}{|c|c|c|}
\hline Stepped analysis & Sponsor's value or assumption & CADTH value or assumption \\
\hline \multicolumn{3}{|c|}{ Correction to sponsor's base case } \\
\hline None & None & None \\
\hline \multicolumn{3}{|c|}{ Changes to derive the CADTH base case ${ }^{a}$} \\
\hline 1. Drug cost of mecasermin & $\begin{array}{l}\text { Cost of mecasermin based on cost per milligram } \\
\text { and exact milligrams needed per year }\end{array}$ & $\begin{array}{l}\text { Cost of mecasermin based on total } \\
\text { number of vials needed per year to cover } \\
\text { estimated number of milligrams }\end{array}$ \\
\hline
\end{tabular}

${ }^{a}$ Changes to derive the CADTH base case under the drug program plan perspective.

Applying the change in Table 15 resulted in a minor increase in the estimated budget impact under the drug plan perspective to $\$ 35,982,122$ over 3 years. The results of the CADTH stepwise reanalyses are presented in summary format in Table 16 and a more detailed breakdown is presented in Table 17.

Table 16: Summary of the CADTH Reanalyses of the Budget Impact Analysis

\begin{tabular}{|l|c|}
\hline Stepped analysis & Three-year total \\
\hline Submitted base case & $\$ 35,486,480$ \\
\hline CADTH base case & $\$ 35,982,122$ \\
\hline
\end{tabular}


Table 17: Detailed Breakdown of the CADTH Reanalyses of the Budget Impact Analysis

\begin{tabular}{|l|c|c|c|c|c|c|}
\hline \multirow{2}{*}{$\begin{array}{l}\text { Stepped analysis } \\
\text { Submitted base } \\
\text { case }^{\mathrm{a}}\end{array}$} & $\begin{array}{c}\text { Scenario } \\
\text { Reference }\end{array}$ & $\begin{array}{c}\text { Year } 0 \text { (current } \\
\text { situation) }\end{array}$ & Year 1 & Year 2 & Year 3 & $\begin{array}{c}\text { Three-year } \\
\text { total }\end{array}$ \\
\cline { 2 - 7 } & New drug & $\$ 0$ & $\$ 0$ & $\$ 0$ & $\$ 0$ & $\$ 0$ \\
\cline { 2 - 7 } & Budget impact & $\$ 0$ & $\$ 11,417,373$ & $\$ 11,867,717$ & $\$ 12,201,390$ & $\$ 35,486,480$ \\
\hline \multirow{3}{*}{ CADTH base case } & Reference & $\$ 0$ & $\$ 0$ & $\$ 0$ & $\$ 0$ & $\$ 0$ \\
\cline { 2 - 7 } & New drug & $\$ 0$ & $\$ 11,572,125$ & $\$ 12,035,169$ & $\$ 12,374,828$ & $\$ 35,982,122$ \\
\cline { 2 - 7 } & Budget impact & $\$ 0$ & $\$ 11,572,125$ & $\$ 12,035,169$ & $\$ 12,374,828$ & $\$ 35,982,122$ \\
\hline
\end{tabular}

Note: CADTH did not undertake any reanalyses and only the sponsor's submitted base case is presented.

CADTH conducted the following additional scenario analyses from the drug plan perspective (Scenarios 1 to 3, Table 18):

1. Assumed that $100 \%$ of patients with SPIGFD patients received a diagnosis and seek treatment.

2. Arbitrarily assumed an increase in the incidence of SPIGFD by $25 \%$.

3. Applied an $92 \%$ reduction in the price of mecasermin to align with the point at which the ICER is within the willingness-to-pay threshold of $\$ 50,000$ per QALY in the CADTH pharmacoeconomic base case.

Table 18: CADTH Scenario Analyses

\begin{tabular}{|l|c|c|c|c|c|c|}
\hline \multirow{2}{*}{$\begin{array}{l}\text { Stepped analysis } \\
\text { CADTH scenario } \\
\text { analysis 1 }\end{array}$} & Budget impact & $\begin{array}{c}\text { Year 0 (current } \\
\text { situation) }\end{array}$ & Year 1 & Year 2 & Year 3 & $\begin{array}{c}\text { Three-year } \\
\text { total }\end{array}$ \\
\cline { 2 - 7 } & Reference & $\$ 0$ & $\$ 0$ & $\$ 0$ & & $\$ 0$ \\
\cline { 2 - 7 } & Budget impact & $\$ 0$ & $\$ 12,160,703$ & $\$ 12,647,299$ & $\$ 13,004,233$ & $\$ 37,812,234$ \\
\hline \multirow{2}{*}{$\begin{array}{l}\text { CADTH scenario } \\
\text { analysis 2 }\end{array}$} & Reference & $\$ 0$ & $\$ 0$ & $\$ 0$ & $\$ 0$ & $\$ 0$ \\
\cline { 2 - 7 } & New drug & $\$ 0$ & $\$ 11,757,457$ & $\$ 12,341,777$ & $\$ 12,820,433$ & $\$ 36,919,667$ \\
\cline { 2 - 7 } & Budget impact & $\$ 0$ & $\$ 11,757,457$ & $\$ 12,341,777$ & $\$ 12,820,433$ & $\$ 36,919,667$ \\
\hline \multirow{2}{*}{$\begin{array}{l}\text { CADTH scenario } \\
\text { analysis 3 }\end{array}$} & Reference & $\$ 0$ & $\$ 0$ & $\$ 0$ & $\$ 0$ & $\$ 0$ \\
\cline { 2 - 7 } & New drug & $\$ 0$ & $\$ 925,770$ & $\$ 962,814$ & $\$ 989,986$ & $\$ 2,878,570$ \\
\cline { 2 - 7 } & Budget impact & $\$ 0$ & $\$ 925,770$ & $\$ 962,814$ & $\$ 989,986$ & $\$ 2,878,570$ \\
\hline
\end{tabular}

Note: All scenario analyses are conducted based on the CADTH base case undertaken from the drug program plan perspective. 